\title{
Phase behaviour of random copolymers and crosslinked homopolymer blends
}

\author{
Dissertation \\ zur Erlangung des Doktorgrades \\ der Mathematisch-Naturwissenschaftlichen Fakultäten \\ der Georg-August-Universität zu Göttingen \\ vorgelegt von \\ Christian Wald \\ aus Bremen
}

Göttingen 2005 
D7

Referentin: Prof. Dr. Annette Zippelius

Korreferent: Prof. Dr. Tim Salditt

Tag der mündlichen Prüfung: 08.11.2005 




\section{Contents}

1. Introduction 1

2. Random copolymer melts 3

2.1. Introduction . . . . . . . . . . . . . . 3

2.2. Polymer models . . . . . . . . . . . . . . . . . 6

2.2.1. Linear homopolymers . . . . . . . . . . . . . . . . . 6

2.2.2. Random block copolymers . . . . . . . . . . . . . 10

2.2.3. Dimensionless representation . . . . . . . . . . . . . . . 12

2.3. Partition function and mean free energy $\ldots \ldots \ldots \ldots$

2.4. Multiphase coexistence ... . . . . . . . . . . . . . . 14

2.4.1. Crushed polymer approximation . . . . . . . . . . . 14

2.4.2. Multi-component picture . . . . . . . . . . . . 15

2.4.3. Quenched average and reduction to a single-chain problem . 16

2.4.4. Effective free energy . . . . . . . . . . . . . . 17

2.4.5. Saddle point approximation . . . . . . . . . . . 18

2.4.6. Flory-Huggins free energy . . . . . . . . . . . . . . . 18

2.4.7. Multiphase equilibria . . . . . . . . . . . . . . . . . . 19

2.4.8. Results . . . . . . . . . . . . . . . . . . . 21

2.5. Inhomogeneous phases . . . . . . . . . . . . . . . . . 21

2.5.1. Order parameters . . . . . . . . . . . . . . . . . 23

2.5.2. Effective free energy . . . . . . . . . . . . . 23

2.5.3. Stability of the homogeneous phase . . . . . . . . 25

2.5.4. Landau expansion . . . . . . . . . . . . . . . . . . . 28

2.5.5. Microphase separation . . . . . . . . . . . . . . 32

2.5.6. Flory-Huggins theory for the charge density . . . . . . . 35

2.5.7. Macrophase separation: Flory-Huggins and Landau theory . 40

2.6. Fractionation . . . . . . . . . . . . . . . . . . 43

2.6.1. Landau free energy . . . . . . . . . . . . . . . . 44

2.6.2. Microphase separation . . . . . . . . . . . . . . . 45

2.6.3. Macroscopic phase separation . . . . . . . . . . . . 45

2.6.4. Free energy of fractionation . . . . . . . . . . . 46

2.6.5. Domain sizes . . . . . . . . . . . . . . . . . . . . . 48

2.6.6. Diblocks . . . . . . . . . . . . . . . . . . . . . 49

2.6.7. Larger block numbers . . . . . . . . . . . . . . . 51 
2.6.8. Implicit fractionation . . . . . . . . . . . . . . . . 52

2.6.9. Summary and discussion . . . . . . . . . . . . . . . 53

2.7. Discussion and conclusions . . . . . . . . . . . . . . 54

3. Crosslinked homopolymer blends 57

3.1. Introduction . . . . . . . . . . . . . . . . . . . 57

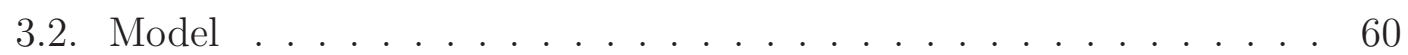

3.2.1. Homopolymer species and incompatibility . . . . . . . . 60

3.2.2. Brownian chain model . . . . . . . . . . . . . . 60

3.2.3. Dimensionless representation, choosing the energy scale . . . 61

3.2.4. Permanent crosslinks and partition function . . . . . . . . 62

3.2.5. Thermal averages in the presence of crosslinks . . . . . . . . 64

3.2.6. Disorder averages and Deam-Edwards distribution . . . . . . 64

3.3. Order parameters . . . . . . . . . . . . . . . . . 66

3.3.1. Gelation order parameter . . . . . . . . . . . . . . . 66

3.3.2. Phase separation order parameter . . . . . . . . . . . 68

3.3.3. Relation to scattering experiments . . . . . . . . . 68

3.4. Derivation of an effective free energy . . . . . . . . . . . . . . . 71

3.4.1. Disorder averaged free energy . . . . . . . . . . . . . . 71

3.4.2. Effective free energy . . . . . . . . . . . . . . 73

3.4.3. Expectation values of the order parameters . . . . . . . . 75

3.4.4. Shifted density field . . . . . . . . . . . . 76

3.5. Analysis . . . . . . . . . . . . . . . . . . . . . . . 77

3.5.1. Mean-field approximation . . . . . . . . . . . . 77

3.5.2. Stability of the homogeneously mixed liquid . . . . . . . 77

3.5.3. Crosslinking in the homogeneously mixed state . . . . . . 78

3.5.4. Stability of the mixed gel . . . . . . . . . . . . . . . 79

3.5.5. Phase diagram . . . . . . . . . . . . . . . . . . . 82

3.5.6. Charge density correlations in the mixed gel . . . . . . . . . 83

3.5.7. Microphase separation . . . . . . . . . . . . . . . . 92

3.5.8. Effects of compositional asymmetry . . . . . . . . . . . 95

3.5.9. Effects of compressibility . . . . . . . . . . . . . . . . 97

3.6. Discussion and conclusions . . . . . . . . . . . . . . . 100

4. Conclusions and outlook 103

\begin{tabular}{ll}
\hline Appendix & 107
\end{tabular}

A. Mathematical tools . . . . . . . . . . . . . . . . . 107

A.1. Replica technique . . . . . . . . . . . . . . 107

A.2. Hubbard-Stratonovich transformations . . . . . . . . . . 108

B. Microphase morphologies . . . . . . . . . . . . . . . . . . . . 109

B.1. Lattice structures . . . . . . . . . . . . . . . . . . . 109

C. Random copolymers: Auxiliary materials . . . . . . . . . . . . . . . 112

C.1. Correlation of remote charges . . . . . . . . . . . . . . . . 112 
C.2. Wiener correlator . . . . . . . . . . . . . . . . . . 114

C.3. Vertex functions . . . . . . . . . . . . . . . . . . . . . 114

C.4. Landau free energy of compressible melts . . . . . . . . . . . 118

C.5. Extended Maxwell construction . . . . . . . . . . . . . . . . 119

C.6. Alternative Landau expansion . . . . . . . . . . . . . . . . . 120

D. Homopolymer blends: Auxiliary materials . . . . . . . . . . . . . 122

D.1. Relation between order parameters and auxiliary fields . . . 122

D.2. Vertex functions . . . . . . . . . . . . . . . . . . 124

D.3. Scaling function for the gelation order parameter . . . . . . 125

D.4. Hessian matrix of the effective free energy . . . . . . . . 126

\section{Bibliography}




\section{Introduction}

The properties desired for practical applications of polymer materials often can not be achieved with a single polymer species. For instance, a common requirement is that of stiffness and high fracture resistance at the same time. Yet, enhancing the stiffness usually leads to more brittle materials. A way to improve the material is to combine different polymer species with complementary properties, for instance glassy and rubbery polymers. This is not straightforward, since different polymer species typically are immiscible. The reason is that in macromolecules the entropy of mixing is reduced by a factor of the degree of polymerisation, so that small energetic incompatibilities suffice to induce phase separation. Therefore, many polymer blends are not perfectly mixed, but reveal droplets of the minority species in the matrix of the majority component. In high-impact polystyrene, for instance, polybutadiene rubber particles dispersed in the matrix of stiff but brittle polystyrene only moderately lower the stiffness but considerably raise the toughness, since the fracture energies are absorbed by yield processes initiated by the rubber particles $[1,2]$. In other cases, superior properties are achieved with a homogeneous blend of miscible polymers. An example is polystyrene blended with poly(phenyleneoxide) [1], which also combines a high modulus with acceptable fracture resistance. Hence the issues of miscibility and the morphology of phase separated states are important for the design of polymeric materials.

In the present thesis we consider two possible ways of enhancing the compatibility of distinct polymer species: copolymerisation and crosslinking. In copolymers, separation is hampered by linking the monomers or prepolymers of both species covalently into hybrid macromolecules. Their miscibility is not unlimited: the typically nonzero width of the distribution of chain compositions still allows for macroscopic phase separation. In addition, the compositional heterogeneity gives rise to the possibility of microphase separation with small-scale composition modulations. The phase behaviour of random block copolymers is discussed in chapter 2 , which also includes a more detailed introduction into this field.

The second way of preventing macrophase separation considered in the present thesis is crosslinking. The introduction of a sufficient number of permanent crosslinks into a well-mixed (homo-)polymer blend generates a network that eventually turns the liquid into a gel, i.e. an amorphous solid. With both species being likewise part of the network, macroscopic phase separation is inhibited. The rigidity of the gel network only allows for local segregation on length-scales approximately up to the mesh size. The phase behaviour of crosslinked homopolymer blends is discussed in chapter 3, including pre-transitional composition fluctuations and the freezing-in of fluctuations by the network. A more detailed introduction is also given there. 


\section{Introduction}




\section{Random copolymer melts}

\subsection{Introduction}

Melts of linear random copolymers, i.e. chains composed of two chemically different species of monomers " $A$ " and " $B$ " in random sequences, exhibit a rich phase behaviour because of the competition between the repulsion of the two species, usually referred to as incompatibility, and the forces favouring the mixed state. Besides the entropy of mixing, which would also be present, e.g., in a homopolymer system, these forces are due to the permanent bonds between $A$ and $B$ in the copolymer that prevent complete separation of the two species. For long chains, the distribution of the fractions of $A$ and $B$ monomers in the chain is typically narrow, hence macroscopic phase separation only yields a small gain in energy. The separation of $A$ and $B$ can, however, also be achieved by the formation of domains on a smaller length-scale; this phenomenon is termed microphase separation.

Systems of regular copolymers, e.g. di- and triblock copolymers, and copolymers blended with homopolymers, have been investigated in detail both theoretically [36] and experimentally [7-11]. These systems are known to phase separate on a mesoscopic scale with a variety of morphologies, e.g. lamellæ, hexagonally ordered cylinders, spherical domains on a bcc lattice or bicontinuous phases [1, 6, 12].

Phase separations in compositionally heterogeneous copolymer systems were first addressed by Scott [13] within a mean-field theory of multi-component demixing based on the Flory-Huggins theory of polymer solutions [14]. The description of the melt is coarse-grained, i.e. it disregards the conformations of the individual chains. Scott calculated the spinodal, i.e. the limit of stability of the homogeneously mixed state against separation into macroscopic phases, for arbitrary distributions of chain compositions. Bauer [15] extended the theory to assess the coexistence of multiple phases. In particular, he suggested an algorithm to compute the equilibrium transition points, which are generally located at smaller incompatibility (i.e. higher temperature) than the spinodal, except in the case of a symmetric composition. His method was applied to the case of random copolymers by Nesarikar et al. [16], who computed the phase diagram for various chain lengths and compositions, and compared them to the results obtained within the Landau approach by Fredrickson et al. [17] (see below).

The first theory of microphase separation in random copolymer melts was reported by Shakhnovich and Gutin [18]. Within a mean-field Landau approach with a microscopic description of the conformational entropy and a phenomenological incompatibility term, they considered a single copolymer chain of infinite 


\section{Random copolymer melts}

length, with symmetric composition and an uncorrelated sequence. Fredrickson et al. [17, 19], starting from a microscopic model, developed a theory for random block copolymers containing a finite number $Q$ of prepolymer blocks of uniform size. The $A-B$ sequences are taken to be Markovian, ranging from alternating chains $(A B A B \ldots)$ via uncorrelated sequences to homopolymeric chains. While the former are predicted to undergo a direct transition from the homogeneous mixture to a microphase separated state, melts of uncorrelated or almost homopolymeric chains reveal an intermediate regime with two coexisting macroscopic phases over a temperature range of the order $1 / Q$. The formation of microphases is studied employing a variational first-harmonic ansatz with four possible morphologies lamellar, hexagonal and bcc lattices, and a superposition of randomly oriented sinusoidal modulations of the local composition. The characteristic wavelength of the microphases is reported to be infinite at the transition for uncorrelated or homopolymeric statistics and finite for alternating chains, and to decrease upon going deeper into the microphase separated region.

The results for macrophase separation into two phases within the multi-component theory and the Landau theory are compared in ref. [16]. While the predictions agree reasonably for melts with symmetric composition containing equal amounts of $A$ and $B$ blocks, for which the transition is of second order within mean-field theory, the results are found to disagree considerably in the asymmetric case, where the transition is of first order. Monte-Carlo simulations by Houdayer and Müller for random copolymer chains $[20,21]$ show major deviations from both mean-field theories, which are partly attributed to the effect of fluctuations. A separation into two homogeneous phases is found only for melts of almost homopolymeric chains, which remix on further decrease in temperature. Due to the limited system size in the simulations, the discrimination between a homogenous mixture and a microphase separated state is difficult. The authors of [21] interpret the remixed state as the coexistence of three phases, two homogeneous phases and a possibly microstructured third phase with symmetric composition, over an extended temperature range, as predicted for $Q=2$, i.e. diblock copolymer/homopolymer blends, by Janert and Schick [4]. For $Q=3$, Houdayer and Müller have analysed the composition of the three phases and found that the melt fractionates according to the chain sequences: while the two macrophases predominantly consist of homopolymeric chains, the third phase is mainly populated by copolymer chains. A theoretical description of the coexistence of two homogeneous phases with an, in contrast to [16], microstructured third phase was given by Subbotin and Semenov [22] for melts having symmetric or nearly symmetric composition within a mean-field Landau approach extending ref. [17]. According to their theory, the melt first splits into two macroscopic phases, from which a lamellar third phase separates. The theory includes the case of slightly asymmetric compositions, for which hexagonal and cubic morphologies are predicted to occur on further cooling.

First accounts of the effect of fluctuations were given in refs. [23, 24]; in contrast to mean-field theory, they predicted that the random copolymer melt was stable against microphase separation. Later it was shown [25], however, that these results 
were due to the neglect of a term in the effective Hamiltonian that had wrongly been considered irrelevant in [17] and [23, 24], with dramatic consequences in the fluctuation theory. For strongly correlated chain sequences, corresponding to large block sizes or strong Markovian correlation, the inclusion of the previously dropped term restores the microphase separation. The transition is rendered weakly firstorder, yet the period and amplitude of the microphases match the mean-field predictions [25]. The jump in the order parameter is reported to decrease with the block size and the correlation parameter, in agreement with the findings of Houdayer and Müller [20, 21]. Using a Ginzburg criterion these authors found that mean-field theory breaks down in a temperature range of the order $Q^{2} / M$ about the transition point, with $M$ denoting the length of the blocks.

A branch of the theory of random copolymers not covered here is the application to the protein folding problem. The native state of the protein is identified with a frozen state of the random copolymer system, in which only a few conformations of the chain are accessible. The freezing transition may or may not precede the microphase transition [26].

The experimental situation is less clear. There is evidence of both separation into macroscopic phases [27, 28] and microphases [29 31]. To our knowledge, the thermodynamic properties of the microphase separation of a well-defined system were, however, reported only recently [30], for the case of randomly grafted copolymers, which is not directly comparable to linear block copolymers (a theory of randomly grafted copolymers is given in [32]). The existence of microphase separation is also well-known for polyurethanes [31, 33]; yet these system reveal a certain complexity going beyond the limits of our theoretical description: besides having a large segmental polydispersity, they contain soft and hard segments, and the formation of microdomains is strongly affected by hydrogen bonding and sometimes crystallisation. Ryan et al. [31] studied the microphase transition in a polyurethane system with suppressed crystallinity, yet with considerable polydispersity in the chain lengths. A rigorous discrimination between the disordered and the microphase separated state is difficult [33].

The starting point of our investigations is the microscopic model for random block copolymers proposed by Fredrickson et al. [17], from which we derive an expression for the disorder-averaged free energy in the replica formulation. To assess the coexistence of multiple homogeneous phases, the free energy is coarse-grained and expressed in terms of a multi-component theory with a separate density for each mean chain composition. This free energy is shown to be identical to the Flory-Huggins free energy of ref. [15] for incompressible melts, so that our equations for the phase equilibria are equivalent to those in ref. [16]. The latter are solved with an implementation of the algorithm suggested in [15].

To study microphase separation, the average free energy is expressed in terms of an effective free energy functional of the local monomer density and the local difference of the concentrations of $A$ and $B$ monomers. The latter is referred to as the charge density by analogy to electric charges, despite of the opposite sign and the different range of the interaction. The effective free energy is expanded 


\section{Random copolymer melts}

in a Landau series in order to carry out the disorder average. The resulting free energy is similar but not equal to that of [17]. Substantial differences arise from certain approximations made in [17] that qualitatively change the microphase separation behaviour: they neglect the structure-dependence of the free energy that, in our theory, allows for determining the favoured morphology of the microphases. Furthermore, these approximations lead to the incorrect prediction that, at their onset, the microphases are characterised by an infinite wavelength (except in melts of predominantly alternating chains). A careful analysis shows that microphases set in with a large but finite length scale.

Comparing the predictions for the coexistence of macroscopic phases within both approaches, multi-component theory and Landau theory, we find agreement for the symmetric case, in accordance with Nesarikar et al. [16]. In the asymmetric case, the transition is of first order, and the truncated Landau series is no longer valid.

To assess fractionation and the coexistence of a microstructured phase with homogeneous phases, we use a caricature of the fractionated state, in which (only) the homopolymeric chains may separate from the rest to constitute two macrophases, coexisting with a possibly microphase separated remaining phase. Keeping the spatial dependence of the free energy only on the quadratic level, we rule out the established mechanism for microphase separation based on the subtle balance between the spatial dependence of the quadratic and quartic terms in the Landau expansion as described, e.g., in ref. [17]. Hence we are able to show that pure fractionation, i.e. the segregation of chains according to their sequences, suffices to promote microphase separation as suggested in ref. [21], independent of the conventional mechanism. Moreover, in contrast to ref. [22], our ansatz allows for the direct investigation of fractionation, since we explicitly account for different chain statistics in different phases.

\subsection{Polymer models}

In the following we shall develop a statistical mechanical model for random copolymer melts. To keep it as simple and tractable as possible, the model will reflect only the essential features of the polymer system. In particular, chemical details of the monomer units and effects of polydispersity, i.e. different chain lengths, are neglected. Moreover, we shall concentrate on the case of linear polymer chains aside from the nonlinear structures produced by crosslinking, which are considered in chapter 3 .

\subsubsection{Linear homopolymers}

Random copolymers and homopolymer blends have some fundamental properties in common with ordinary homopolymers, hence it is useful to start with a model for simple homopolymers as a basis for the description of random copolymers in section 2.2.2, and of crosslinked homopolymer blends in section 3.2 . 


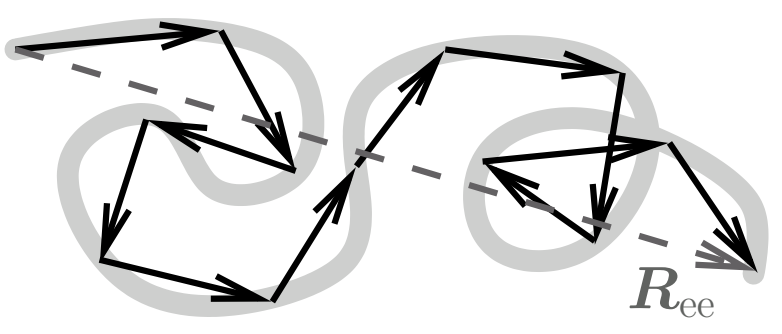

Figure 2.1: Coarse-graining of a polymer chain. The original contour (thick line) is approximated by a chain of straight segments (solid arrows). The dashed arrow indicates the end-to-end vector $\boldsymbol{R}_{\mathrm{ee}}$.

\section{Chain connectivity}

The most prominent feature of polymers is their connectivity, i.e. the fact that the monomer units are linked into macromolecules. Out of the various possible topologies like trees or stars we pick the simplest one, namely linear chains, consisting of bifunctional monomers binding to (at most) two other monomer units.

On a small length-scale, the geometrical and other properties of the polymer strongly depend on the chemical details of the monomer species. The positions and orientations of neighbouring units in the chain are correlated due to usually quite restricted bond angles and bond lengths, and less rigid, but still constrained bond rotation angles. Typically, the amplitudes of the thermal oscillations of bond lengths and angles are of the order of $3 \%$ and $3-5 \%$, respectively [34], and can often be neglected. The potentials for bond rotation angles generally exhibit multiple minima, three in the case of single bonds between tetravalent carbon atoms. The energy barriers in-between are typically larger than $k_{\mathrm{B}} T$, yet small enough to allow for frequent transitions between the possible states [34], giving rise to a diversity of possible chain configurations that grows large with the number of rotating bonds. In turn, the correlations between two monomers decay with their distance along the chain contour [1, 34-36], so that the details of the monomer properties are effectively masked on a larger length-scale. The decay length, the persistence length, is a measure for the stiffness of the polymer.

A coarse-grained picture allowing for the calculation of distribution functions of, e.g., the end-to-end distance of the chain, can be obtained as follows [1, 14, 35]: The polymer chain is divided into $L$ pieces of equal number of subunits or arclength $l$, and their endpoints are connected with straight segments having a mean square length $a^{2}$; this procedure is illustrated in fig. 2.1. Provided $l$ is much larger than the persistence length, the orientations of the segments are practically uncorrelated, so that the contour of the segment chain formally equals the path of a Brownian particle or a random walk ${ }^{1}$. Consequently, the distance vector of two segments approximately follows a Gaussian distribution, with a mean square distance proportional to the number of segments in-between. For instance, the mean square end-to-end distance of the segment chain is given by $R_{\mathrm{ee}}^{2}:=\left\langle R^{2}\right\rangle=L a^{2}$. In the construction, the segment size $a$ is chosen such that the mean square end-to-

\footnotetext{
1 Strictly speaking, this only holds true if the volume occupied by the monomers can be ne-
} glected, since the polymer chains cannot overlap. This issue will be discussed in section 2.2.1. 


\section{Random copolymer melts}

end distances of the segmented and the real chain coincide. This also holds for the radius of gyration $R_{\mathrm{g}}$, an alternative measure for the chain extension appearing in the low momentum expansion of the scattering factor of single polymer chains. It is defined as the mean square distance between the monomers, i.e.

$$
R_{\mathrm{g}}^{2}:=\frac{1}{2 n^{2}} \sum_{i, j=1}^{n}\left\langle\left(\boldsymbol{r}_{i}-\boldsymbol{r}_{j}\right)^{2}\right\rangle
$$

for a linear chain of $n$ monomers located at $\boldsymbol{r}_{i}$, where $i=1, \ldots, n$.

Up to this point, the choice of the number of segments $l$ is arbitrary, in the range from the persistence length to the arclength of the polymer, and the segment size $a$ is determined depending on $l$. This arbitrariness can be removed by requiring not only the average coil size, but also the length of the fully extended chain (roughly speaking the arclength) to be the same for the real polymer $\left(R_{\max }\right)$ and the segmented one $(L a)$. (The average size of the individual segments is assumed to be unaffected by the stretching of the chain.) It follows that $a$ is given by the Kuhn length $a_{\mathrm{K}}:=\left\langle R^{2}\right\rangle / R_{\max }$, which, like the persistence length, is a measure for the stiffness of the polymer. In fact, both quantities take similar values [1].

The Gaussian approximation made above can be tested explicitly for simple models like the freely jointed chain, in which adjacent monomers are linked by bonds having fixed length and random orientation. It turns out that the approximate distribution of the end-to-end distance, even for a chain as short as five units, is in good agreement with the exact result [1]. Hence it seems well justified to employ the Gaussian chain model, which offers invaluable advantages in the analytical treatment.

\section{Gaussian model}

To parametrise the model for Gaussian chains, we consider a melt of $N$ polymer chains containing $L$ segments each, thus neglecting effects of polydispersity. The position of segment $s$ on chain $i$ in the $d$-dimensional space is denoted as $\boldsymbol{R}_{i}(s) \in \mathbb{R}^{d}$ where $i=1, \ldots, N$ and $s=1, \ldots, L$. The distance vector between neighbouring segments on the chain, in thermal equilibrium, is taken to follow a Gaussian distribution with zero average and a root mean square length of $b$, which can be interpreted as the Kuhn length of the polymer. The chain configuration is described by the Wiener Hamiltonian

$$
H^{\mathrm{W}}:=k_{\mathrm{B}} T \cdot \frac{d}{2 b^{2}} \sum_{i=1}^{N} \sum_{s=1}^{L-1}\left(\boldsymbol{R}_{i}(s+1)-\boldsymbol{R}_{i}(s)\right)^{2} .
$$

The pre-factor of $k_{\mathrm{B}} T$ in eq. (2.2), which cancels out with the factor of $1 / k_{\mathrm{B}} T$ in the Boltzmann weight and yields a temperature-independent partition function, reflects the entropic nature of the distribution of chain configurations.

In the case of Gaussian chains, the radius of gyration defined in eq. (2.1) (with $L$ segments instead of $n$ monomers) can be calculated analytically. The mean square 
distance of two monomers $i$ and $j$ of the same chain is given by $\left\langle\left(\boldsymbol{R}_{i}-\boldsymbol{R}_{j}\right)^{2}\right\rangle=$ $|i-j| a^{2}$, hence $R_{\mathrm{g}}=L a^{2} / 6$ (up to corrections of the order $L^{-1}$, which are due to not fully accounting for the segments at the chain ends). To indicate the dependence on the chain length, the radius of gyration will be also referred to as $R_{L}$. Obviously, the average end-to-end distance and the radius of gyration of the Gaussian chain are related via $R_{\mathrm{ee}}=\sqrt{6} R_{L}$.

\section{Excluded volume}

The analogy of the polymer contour with a random walk, used to derive the ideal chain model in the previous section, must be taken with a pinch of salt. It had been obtained considering only short-range interactions of subunits being neighbours on the polymer chain, disregarding the long-range intramolecular interactions of subunits that are close in space, yet distant along the chain contour.

In the case of homopolymers, the most important example is the steric hindrance or interference: In contrast to the path of a Brownian particle, a polymer chain may not cross itself, since the subunits occupy a certain volume, the excluded volume. For long chains, many of the configurations of the ideal chain predicted by the Gaussian model are ruled out. Since extended configurations are more likely to "survive", it appears that the mean extension of real chains must be larger than predicted. This is indeed the case for dilute polymer solutions, especially in a good solvent, which interacts more favourably with the polymer than the polymer itself, thus amplifying the interference effect and leading to polymer swelling. Conversely, the effect is counteracted by poor solvents. In some systems, the effective attraction due to the poor solvent is comparable to the self-repulsion of the polymer, depending on temperature. At the $\theta$ temperature, in an ideal solution, the two interactions just cancel, and the polymer chains adopt the configurations of the ideal Gaussian chain.

Measurements and theoretical considerations show that in polymer melts the chain configurations are also nearly ideal, with only small distortions [1, 14]. This is because of the intermolecular forces not taken into account so far: for the interference of two portions of polymer, it is irrelevant whether they belong to the same chain or not, and there is no reason to discriminate between the according intermolecular and long-range intramolecular interactions. In a polymer melt, the chains could avoid self-interference by assuming a more extended shape, but only at the cost of more frequent collisions with other chains. Therefore, the shape and size of coils effectively remain unchanged despite steric hindrance.

Nevertheless, it is necessary to account for the excluded volume effect in the model as a means of limiting compressibility, since the copolymer systems discussed in the present thesis could break down in the absence of repulsive forces: In a copolymer melt, the attraction between monomers of the same kind could induce the collapse onto one point in space per species. In a crosslinked melt, the network favour the contraction onto a single point. To model steric repulsion we shall use 
the usual excluded volume interaction [14], adapted to polymer melts,

$$
H^{\mathrm{EV}}:=k_{\mathrm{B}} T \cdot \frac{\mu}{2} \sum_{i_{1,2}=1}^{N} \sum_{s_{1,2}=1}^{L} U\left(\boldsymbol{R}_{i_{1}}\left(s_{1}\right)-\boldsymbol{R}_{i_{2}}\left(s_{2}\right)\right),
$$

with a control parameter $\mu$ and short-ranged function $U(|R|)$ normalised according to $\int \mathrm{d} V U(|R|)=1$ and mostly idealised by a delta-shaped function throughout this thesis. The pre-factor $k_{\mathrm{B}} T$ reflects the entropic (or steric) nature of the volume exclusion. Essentially, the Hamiltonian (2.3) counts and penalises each contact of monomers with an energy proportional to $\mu \cdot k_{\mathrm{B}} T$.

\subsubsection{Random block copolymers}

As opposed to homopolymer chains, copolymers are composed of different types of subunits. We consider a melt of linear random block copolymers built of two monomer species, denoted as " $A$ " and " $B$ ", that energetically tend to demix. Provided the block length is larger than the Kuhn length, the coarse-grained Gaussian model from section 2.2 .1 can be supplemented, without loss of generality, to account for the non-uniform composition of the chains. The resulting model is identical to the one proposed in [17] by Fredrickson et al..

\section{Monomer species and incompatibility}

Each monomer in the melt is assigned an attribute

$$
q_{i}(s):= \begin{cases}+1, & \text { monomer } s \text { on chain } i \text { is of type } A \\ -1, & \text { monomer } s \text { on chain } i \text { is of type } B\end{cases}
$$

indicating its type. These $q_{i}(s)$ will be referred to as the monomer charges. The incompatibility of $A$ and $B$ monomers can be modelled with a Hamilton function similar to the Coulomb interaction of electric charges, yet with opposite sign to penalise contacts of $A$ and $B$ monomers:

$$
H^{\mathrm{C}}:=-\frac{\chi}{2} \sum_{i_{1,2}=1}^{N} \sum_{s_{1,2}=1}^{L} q_{i_{1}}\left(s_{1}\right) q_{i_{2}}\left(s_{2}\right) \cdot W\left(\left|\boldsymbol{R}_{i_{1}}\left(s_{1}\right)-\boldsymbol{R}_{i_{2}}\left(s_{2}\right)\right|\right)
$$

Each pair of monomers yields an attractive or repulsive contribution, depending on whether they are of the same species or not, weighted with respect to their distance. The weight $W(R)$ is assumed to decay rapidly with $R$ to yield a local interaction, and it is taken to be normalised according to $\int \mathrm{d} V W(|R|)=1$. Throughout this thesis, $W(R)$ will mostly be idealised to be delta-shaped. The incompatibility parameter $\chi>0$ sets the overall scale of the interaction, and it is the main control parameter of the system, determining the phase state of the copolymer melt.

Aside from the incompatibility (2.5), the monomers of both types are assumed to have identical properties, so that the chain connectivity and the excluded volume interaction from section 2.2 .1 can be used without modification. 


\section{Chain sequences}

To complete the definition of the model it is necessary to precisely specify the kind of randomness determining the sequences of $A$ and $B$ along the chains. The sequences are not subjected to thermal equilibration, since the chains are (co-)polymerised via covalent bonds, which are stable on the time scales of the mixing and demixing processes (and far beyond). Hence the charges $q_{i}(s)$ indicating the monomer species are quenched random variables.

In our model, each copolymer chain is composed of $Q$ homopolymeric blocks which, for simplicity, are taken to have the same length $M$ regardless of their type, hence $L=M Q$. Due to the blockiness, $q_{i}(s)=q_{i}\left(s^{\prime}\right)$ for monomers $s$ and $s^{\prime}$ residing on the same block.

We confine ourselves to a stationary copolymerisation process, in which the copolymerisation rates may depend on the species of the newly added block and the actual end of the growing chain, but are constant in time. This corresponds to steady reaction conditions, in particular with continuous replenishment of consumed monomers. The $A / B$ block sequences can then be modelled as Markov chains with a (constant) transition matrix

$$
\mathbf{M}:=\left(\begin{array}{cc}
p_{A A} & p_{B A} \\
p_{A B} & p_{B B}
\end{array}\right)
$$

Its elements indicate the conditional probabilities $p_{a a^{\prime}}$ (with $a, a^{\prime} \in\{A, B\}$ ) that a block of a given type $a$ is followed by a block of type $a^{\prime}$ in the chain. The probabilities are related to the rate constants $k_{a a^{\prime}}$ via $p_{a a^{\prime}} \propto k_{a a^{\prime}} c_{a^{\prime}}$, with the concentration $c_{a^{\prime}}$ of monomer $a^{\prime}$ in the solution. Furthermore, we assume that $A$ and $B$ monomers start new growing chains in the same ratio as they would occur in an infinite chain, so that the average composition is constant over the whole length of the chains rather than following a gradient. In the simple case that $k_{A B}=k_{B A}$, this means that $A$ and $B$ blocks are initiated in proportion to their abundance in the solution, i.e. by an initiator being "blind" for the species. With this assumption, the chain sequences can be described by a stationary Markov chain.

As $p_{A B}=1-p_{A A}$ and $p_{B A}=1-p_{B B}$ due to the normalisation of probability, $\mathbf{M}$ has only two independent entries. The eigenvalues of $\mathbf{M}$ are unity and

$$
\lambda:=p_{A A}-p_{B A}=p_{B B}-p_{A B} .
$$

The respective eigenvectors read $(p, 1-p)^{T}$, corresponding to the stationary process with an overall fraction

$$
p:=\frac{p_{B A}}{p_{A B}+p_{B A}}
$$

of $A$ monomers in the final copolymer, and $(-1,+1)$ corresponding to the nonstationary part. We parametrise the matrix in terms of the overall composition $p$ and the eigenvalue $\lambda$, which determines the average correlation along the chain. 
It ranges from $\lambda=+1$ leading to homopolymeric chains (" $A A \ldots$ " or " $B B \ldots$. ), via $\lambda=0$ for uncorrelated chains, to $\lambda=-1$ corresponding to alternating chains (" $A B A B \ldots$..). Strictly alternating chains, however, require a symmetric composition, i.e. equal amounts of $A$ and $B$ in the final polymer. In general, the range of $\lambda$ depends on $p$ via

$$
1-\frac{1}{\max (p, 1-p)} \leq \lambda \leq 1
$$

Charges on distinct chains are taken to be uncorrelated, so the probability distribution $P\left(\left\{q_{i}(s)\right\}\right)$ of sequences in the melt factorises into single-chain distributions $p$,

$$
P\left(\left\{q_{i}(s)\right\}\right):=\prod_{j=1}^{N} p\left(\left\{q_{j}(s)\right\}\right)
$$

with $p\left(\left\{q_{i}(s)\right\}\right)$ defined by the above considerations. The chain sequences enter the calculation in the form of moments of the charges $q_{i}(s)$ with respect to the disorder average

$$
[\cdots]:=\sum_{\left\{q_{i}(s)\right\}} \cdots P\left(\left\{q_{i}(s)\right\}\right)
$$

over the chain sequences. The lowest moment is the average composition

$$
q:=\left[q_{i}(s)\right]=2 p-1
$$

the higher moments $\left[q_{i_{1}}\left(s_{1}\right) q_{i_{2}}\left(s_{2}\right)\right]$ etc. are computed in appendix C.1.

\subsubsection{Dimensionless representation}

For convenience, we measure all length-scales in essentially units of the segment size and introduce dimensionless positional variables

$$
\boldsymbol{r}_{i}(s)=\frac{\sqrt{2 d}}{b} \boldsymbol{R}_{i}(s)
$$

The volume of the sample is rescaled as $V^{\prime}=\left(2 d / b^{2}\right)^{d / 2} V$, and we introduce the overall density

$$
\varrho_{0}^{\prime}:=N L / V^{\prime} .
$$

The normalisation of $U$ and $W$, rescaled similarly, can be restored by introducing $U^{\prime}(r):=\left(b^{2} / 2 d\right)^{d / 2} U(R)$ and $W^{\prime}(r):=\left(b^{2} / 2 d\right)^{d / 2} W(R)$, which generates a prefactor of $V^{\prime} / V$ in the excluded volume and incompatibility interaction. It can be absorbed into rescaled interaction parameters

$$
\mu=: \frac{V}{2 V^{\prime}} \cdot \mu^{\prime} \quad \text { and } \quad \chi=: \frac{V}{2 V^{\prime}} \cdot k_{\mathrm{B}} T \cdot \chi^{\prime},
$$


where the irrelevant artificial factors of 2 are introduced to match the convention in the literature. The new parameter $\chi^{\prime} \propto 1 / T$ is chosen such that the incompatibility Hamiltonian is formally proportional to $k_{\mathrm{B}} T$ like $H^{\mathrm{W}}$ and $H^{\mathrm{EV}}$, so the Boltzmann weight is independent of $T$. We choose the energy scale such that $k_{\mathrm{B}} T=1$; "cooling" towards demixing is then accomplished, instead of by lowering $T$, by raising $\chi$, which therefore will also be referred to as the "inverse temperature". To keep the notation simple, we drop the primes,

$$
V^{\prime} \rightarrow V, \quad \varrho_{0}^{\prime} \rightarrow \varrho_{0}, \quad U^{\prime} \rightarrow U, \quad W^{\prime} \rightarrow W, \quad \mu^{\prime} \rightarrow \mu, \quad \chi^{\prime} \rightarrow \chi,
$$

so that, expressed in the new variables, the Wiener Hamilton and the Hamiltonians of excluded volume and incompatibility read

$$
\begin{aligned}
H^{\mathrm{W}} & =\frac{1}{4} \sum_{i=1}^{N} \sum_{s=1}^{L-1}\left(\boldsymbol{r}_{i}(s+1)-\boldsymbol{r}_{i}(s)\right)^{2} \\
H^{\mathrm{EV}} & =\frac{\mu}{4} \sum_{i_{1,2}=1}^{N} \sum_{s_{1,2}}^{L} U\left(\left|\boldsymbol{r}_{i_{1}}\left(s_{1}\right)-\boldsymbol{r}_{i_{2}}\left(s_{2}\right)\right|\right)
\end{aligned}
$$

and

$$
H^{\mathrm{C}}=-\frac{\chi}{4} \sum_{i_{1,2}=1}^{N} \sum_{s_{1,2}}^{L} q_{i_{1}}\left(s_{1}\right) q_{i_{2}}\left(s_{2}\right) W\left(\left|\boldsymbol{r}_{i_{1}}\left(s_{1}\right)-\boldsymbol{r}_{i_{2}}\left(s_{2}\right)\right|\right) .
$$

The total Hamiltonian is denoted as $H^{\mathrm{T}}:=H^{\mathrm{W}}+H^{\mathrm{EV}}+H^{\mathrm{C}}$.

\subsection{Partition function and mean free energy}

The central quantity to derive the properties of the random copolymer melt in thermal equilibrium is the partition function, which, for a given realisation $\left\{q_{i}(s)\right\}$ of chain sequences, reads

$$
Z\left(\left\{q_{i}(s)\right\}\right)=\int \mathcal{D} \boldsymbol{r}_{i}(s) \exp \left(-H^{\mathrm{T}}\left(\left\{r_{i}(s)\right\},\left\{q_{i}(s)\right\}\right)\right)
$$

where $\int \mathcal{D} \boldsymbol{r}_{i}(s)$ denotes the integral over all chain configurations. As we are interested in the typical behaviour of random copolymers, it is, besides being practically impossible, also pointless to consider a particular realisation of sequences. Moreover, the melt is assumed to be self-averaging in the thermodynamic limit, so it appears natural to compute the disorder-averaged free energy

$$
F:=-\left[\ln Z\left(\left\{q_{i}(s)\right\}\right)\right]=\lim _{n \rightarrow 0} \frac{1-Z_{n}}{n},
$$


with the sequences average (2.11). Using the replica-trick (see appendix A.1), the average of the logarithm has been expressed in terms of disorder averaged powers of $Z$,

$$
Z_{n}:=\left[\left(Z\left(\left\{q_{i}(s)\right\}\right)\right)^{n}\right] .
$$

For integer $n, Z^{n}$ can be interpreted as the partition function of $n$ independent copies (replicas) of the original melt, with, however, identical chain sequences. With replicated positional variables $\boldsymbol{r}_{i}^{\alpha}(s)$, enumerated by the replica index $\alpha=$ $1, \ldots, n$, and with the replicated total Hamiltonian

$$
\begin{aligned}
& H_{n}^{\mathrm{T}}\left(\left\{\boldsymbol{r}_{i}^{\alpha}(s)\right\},\left\{q_{i}(s)\right\}\right):= \\
& \qquad \sum_{\beta=1}^{n} H^{\mathrm{W}}\left(\left\{\boldsymbol{r}_{i}^{\beta}(s)\right\}\right)+H^{\mathrm{EV}}\left(\left\{\boldsymbol{r}_{i}^{\beta}(s)\right\}\right)+H^{\mathrm{C}}\left(\left\{\boldsymbol{r}_{i}^{\beta}(s)\right\},\left\{q_{i}(s)\right\}\right),
\end{aligned}
$$

we obtain the disorder-averaged replicated partition function

$$
Z_{n}=\left[\int \mathcal{D} \boldsymbol{r}_{i}^{\alpha}(s) \exp \left(-H_{n}^{\mathrm{T}}\left(\left\{\boldsymbol{r}_{i}^{\alpha}(s)\right\},\left\{q_{i}(s)\right\}\right)\right)\right]
$$

\subsection{Multiphase coexistence}

One approach to the phase diagram of random copolymers is based on the idea of a multi-component mixture: the chains are divided into components according to the total content of $A$-monomers per chain. For a given number $Q$ of blocks, this content ranges from $0 \cdot M$ to $Q \cdot M$, yielding $(Q+1)$ different species. Within this picture of a multi-component fluid, the phase diagram consists of a series of phase separation transitions into more and more macroscopic phases, up to $(Q+1)$ according to Gibbs' phase rule. These transitions have been worked out by Nesarikar et al. [16] following Bauer's approach [15], which is based on the Flory-Huggins theory [37-40] for polymer solutions.

In the following we shall derive the free energy used in ref. [15] and the multicomponent phase diagram from our microscopic model. Besides providing a microscopic derivation of the theory, this also establishes the link between the coarsegrained multi-component theory and the full microscopic theory discussed later.

\subsubsection{Crushed polymer approximation}

For the discussion of phase separation into homogeneous phases, implying concentration changes on macroscopic length-scales, fluctuations on length-scales smaller than the radius of gyration $R_{L}$ of a single chain are expected to be negligible. Hence we expand the monomer positions about one of the end points

$$
\boldsymbol{r}_{i}^{\alpha}:=\boldsymbol{r}_{i}^{\alpha}(s=1)
$$


yielding

$$
\boldsymbol{r}_{i}^{\alpha}(s)=: \boldsymbol{r}_{i}^{\alpha}+\Delta \boldsymbol{r}_{i}^{\alpha}(s) \quad \forall s .
$$

In the crushed polymer approximation, we let $\Delta \boldsymbol{r}_{i}^{\alpha}(s)=0$, so the Wiener Hamiltonian $H^{W}$ vanishes and we only need to integrate over a single position vector per chain. Eq. (2.23) then becomes

$$
Z_{n}=\left[\int \mathcal{D} \boldsymbol{r}_{i}^{\alpha} \exp \left(-H_{n}^{\mathrm{EV}}\left(\left\{\boldsymbol{r}_{i}^{\alpha}\right\}\right)-H_{n}^{\mathrm{C}}\left(\left\{\boldsymbol{r}_{i}^{\alpha}\right\},\left\{q_{i}\right\}\right)\right)\right]
$$

with $q_{i}=\frac{1}{L} \sum_{s=1}^{L} q_{i}(s)$ denoting the mean monomer charge of chain $i$ as defined in eq. (2.31) and with the integral measure $\mathcal{D} \boldsymbol{r}_{i}^{\alpha}:=\prod_{\alpha=0}^{n} \prod_{i=1}^{N} \mathrm{~d}^{d} r_{i}^{\alpha}$. The replicated Hamiltonians of the excluded volume and incompatibility interaction in the crushed polymer approximation are given by

$$
H_{n}^{\mathrm{EV}}=\frac{\mu L^{2}}{4} \sum_{\alpha=1}^{n} \int \mathrm{d}^{d} x\left(\sum_{i_{1}=1}^{N} \delta\left(\boldsymbol{x}-\boldsymbol{r}_{i_{1}}^{\alpha}\right)\right)\left(\sum_{i_{2}=1}^{N} \delta\left(\boldsymbol{x}-\boldsymbol{r}_{i_{2}}^{\alpha}\right)\right)
$$

and

$$
H_{n}^{\mathrm{C}}=-\frac{\chi L^{2}}{4} \sum_{\alpha=1}^{n} \int \mathrm{d}^{d} x\left(\sum_{i_{1}=1}^{N} q_{i_{1}} \delta\left(\boldsymbol{x}-\boldsymbol{r}_{i_{1}}^{\alpha}\right)\right)\left(\sum_{i_{2}=1}^{N} q_{i_{2}} \delta\left(\boldsymbol{x}-\boldsymbol{r}_{i_{2}}^{\alpha}\right)\right)
$$

where we have replaced the short-ranged functions $U(r)$ and $W(r)$ by delta functions, consistent with the neglect of small-scale fluctuations.

\subsubsection{Multi-component picture}

The partition function in the crushed polymer approximation contains the monomer charges only summed over entire chains, see eq. (2.28). This naturally leads to the multi-component picture, in which the chains are divided into $(Q+1)$ classes $l \in\{0, \ldots, Q\}$ according to their mean charge $L \cdot \mathfrak{q}_{l}$ with

$$
\mathfrak{q}_{l}:=\frac{2 l-Q}{Q}
$$

The replicated monomer density of component $l$ in the $\alpha^{\text {th }}$ replica reads

$$
\rho_{l}^{\alpha}(\boldsymbol{x}):=L \sum_{i=1}^{N} \delta_{q_{i}, \mathfrak{q}_{l}} \cdot \delta\left(\boldsymbol{x}-\boldsymbol{r}_{i}^{\alpha}\right),
$$

where

$$
q_{i}:=\frac{1}{L} \sum_{s=1}^{L} q_{i}(s) \in\left\{-1,-1+\frac{2}{Q}, \ldots, 1\right\}
$$


denotes the charge of chain $i$ divided by $L$, i.e. the mean monomer charge of chain $i$. Due to the blockiness of the copolymer sequences, the possible values of $q_{i}$ differ by multiples of $2 M / L=2 / Q$.

The component densities are introduced as order parameters by functional integration of $Z_{n}$ over $\left\{\rho_{l}^{\alpha}(\boldsymbol{x})\right\}$, fixing their physical values (2.30) with the aid of delta-functions:

$$
\begin{aligned}
Z_{n} \equiv \int \mathcal{D} \rho_{l}^{\alpha} Z_{n} \cdot \delta\left(\rho_{l}^{\alpha}(\boldsymbol{x})-L \sum_{i=1}^{N} \delta_{q_{i}, \mathfrak{q}_{l}} \cdot \delta\left(\boldsymbol{x}-\boldsymbol{r}_{i}^{\alpha}\right)\right) \\
\equiv \int \mathcal{D} \rho_{l}^{\alpha} \mathcal{D} \hat{\rho}_{l}^{\alpha}(\boldsymbol{x}) Z_{n} \cdot \exp \left\{\mathrm{i} \sum_{\alpha=1}^{n} \sum_{l=0}^{Q} \int \mathrm{d}^{d} x \hat{\rho}_{l}^{\alpha}(\boldsymbol{x})\right. \\
\left.\times\left(\rho_{l}^{\alpha}(\boldsymbol{x})-L \sum_{i=1}^{N} \delta_{q_{i}, \mathfrak{q}_{l}} \cdot \delta\left(\boldsymbol{x}-\boldsymbol{r}_{i}^{\alpha}\right)\right)\right\}
\end{aligned}
$$

In the second step, the delta-functions have been expressed by their integral representation with auxiliary fields $\left\{\hat{\rho}_{l}^{\alpha}(\boldsymbol{x})\right\}$ as integrational variables. The measures $\mathcal{D} \rho_{l}^{\alpha}$ and $\mathcal{D} \hat{\rho}_{l}^{\alpha}$ comprise the integrations over $\rho_{l}^{\alpha}(\boldsymbol{x})$ and $\hat{\rho}_{l}^{\alpha}(\boldsymbol{x})$ for all $\alpha=1, \ldots, n$ and $l=0, \ldots, Q$. The introduction of the order parameters will help to reduce the many-chain to a single-chain problem and to integrate out the microscopic degrees of freedom later. For simplicity, we restrict our consideration to the incompressible limit, where the monomer density is spatially homogeneous, given by $L \sum_{i=1}^{N} \delta\left(\boldsymbol{x}-\boldsymbol{r}_{i}\right)=\varrho_{0}$, so that the excluded volume interaction (2.28) just contributes a constant $n \mu L^{2} \varrho_{0}^{2} V / 4$. Omitting this irrelevant constant we arrive at

$$
\begin{aligned}
& Z_{n}=\int \mathcal{D} \rho_{l}^{\alpha} \mathcal{D} \hat{\rho}_{l}^{\alpha} \mathcal{D} \boldsymbol{r}_{i}^{\alpha}\left[\exp \left\{-\mathrm{i} L \sum_{i, l, \alpha} \delta_{q_{i}, \mathfrak{q}_{l}} \hat{\rho}_{l}^{\alpha}\left(\boldsymbol{r}_{i}^{\alpha}\right)\right\}\right] \\
& \times \exp \left\{\mathrm{i} \sum_{\alpha=1}^{n} \sum_{l=0}^{Q} \int \mathrm{d}^{d} x \hat{\rho}_{l}^{\alpha}(\boldsymbol{x}) \rho_{l}^{\alpha}(\boldsymbol{x})+\frac{\chi}{4} \sum_{\alpha=1}^{n} \sum_{l, l^{\prime}=0}^{L} \mathfrak{q}_{l} \mathfrak{q}_{l^{\prime}} \int \mathrm{d}^{d} x \rho_{l}^{\alpha}(\boldsymbol{x}) \rho_{l^{\prime}}^{\alpha}(\boldsymbol{x})\right\} .
\end{aligned}
$$

\subsubsection{Quenched average and reduction to a single-chain problem}

In the following, we shall carry out the disorder average and reduce the many-chain problem to a single-chain problem. To average over the charges of the chains we define the probability

$$
p_{l}:=\mathbb{P}\left(q_{i}=\mathfrak{q}_{l}\right)
$$

that a chain contains exactly $l$ blocks of type $A$. It is computed according to the chain statistics of section 2.2.2. (In the case of uncorrelated sequences $(\lambda=0)$, for example, the number of $A$ blocks follows a binomial distribution with parameters 
$p=(1+q) / 2$ and $Q$.$) Since the compositions of distinct chains are independent,$ the charge average in eq. (2.33) factorises:

$$
\left[\exp \left\{-\mathrm{i} L \sum_{i, l, \alpha} \delta_{q_{i}, \mathfrak{q}_{l}} \hat{\rho}_{l}^{\alpha}\left(\boldsymbol{r}_{i}^{\alpha}\right)\right\}\right]=\prod_{i=1}^{N}\left(\sum_{l=0}^{Q} p_{l} \exp \left\{-\mathrm{i} L \sum_{\alpha=1}^{n} \hat{\rho}_{l}^{\alpha}\left(\boldsymbol{r}_{i}^{\alpha}\right)\right\}\right),
$$

which allows for reducing $Z_{n}$ to a single chain partition function,

$$
\begin{aligned}
& Z_{n}=\int \mathcal{D} \rho_{l}^{\alpha} \mathcal{D} \hat{\rho}_{l}^{\alpha}\left(\sum_{l=0}^{Q} p_{l} \prod_{\alpha=1}^{n} \int \mathrm{d}^{d} x \exp \left\{-\mathrm{i} L \hat{\rho}_{l}^{\alpha}(\boldsymbol{x})\right\}\right)^{N} \\
& \times \exp \left\{\mathrm{i} \sum_{\alpha=1}^{n} \sum_{l=0}^{Q} \int \mathrm{d} d x \hat{\rho}_{l}^{\alpha}(\boldsymbol{x}) \rho_{l}^{\alpha}(\boldsymbol{x})+\frac{\chi}{4} \sum_{\alpha=1}^{n} \sum_{l, l^{\prime}=0}^{L} \mathfrak{q}_{l} \mathfrak{q}_{l^{\prime}} \int \mathrm{d}^{d} x \rho_{l}^{\alpha}(\boldsymbol{x}) \rho_{l^{\prime}}^{\alpha}(\boldsymbol{x})\right\} .
\end{aligned}
$$

\subsubsection{Effective free energy}

The most favourable state of a macroscopically segregating melt should be at most weakly degenerate, so it is safe to assume replica-symmetric order parameters, $c f$. appendix A.1. With

$$
\begin{aligned}
\hat{\rho}_{l}^{\alpha}(\boldsymbol{x}) & =\hat{\rho}_{l}(\boldsymbol{x}) \\
\rho_{l}^{\alpha}(\boldsymbol{x}) & =\rho_{l}(\boldsymbol{x}),
\end{aligned}
$$

the partition function can be expressed as

$$
Z_{n}:=\int \mathcal{D} \rho_{l} \mathcal{D} \hat{\rho}_{l} \exp \left\{-n \mathcal{F}\left(\left\{\rho_{l}(\boldsymbol{x}), \hat{\rho}_{l}(\boldsymbol{x})\right\}\right)+\mathcal{O}\left(n^{2}\right)\right\}
$$

in terms of the effective free energy $\mathcal{F}$. The latter is given by

$$
\begin{aligned}
& \mathcal{F}\left(\left\{\rho_{l}(\boldsymbol{x}), \hat{\rho}_{l}(\boldsymbol{x})\right\}\right):= \\
& \quad-\mathrm{i} \sum_{l=0}^{Q} \int \mathrm{d}^{d} x \hat{\rho}_{l}(\boldsymbol{x}) \rho_{l}(\boldsymbol{x})-\frac{\chi}{4} \sum_{l, l^{\prime}=0}^{Q} \mathfrak{q}_{l} \mathfrak{q}_{l^{\prime}} \int \mathrm{d}^{d} x \rho_{l}(\boldsymbol{x}) \rho_{l^{\prime}}(\boldsymbol{x})-N \sum_{l=0}^{Q} p_{l} \ln a_{l}
\end{aligned}
$$

where

$$
a_{l}:=\int \mathrm{d}^{d} x \exp \left\{-i L \hat{\rho}_{l}(\boldsymbol{x})\right\}
$$

In deriving eqs. (2.39) and (2.40) we have used that $\ln \left(\sum_{l} p_{l}\left(a_{l}\right)^{n}\right)=n \sum_{l} p_{l} \ln \left(a_{l}\right)+$ $\mathcal{O}\left(n^{2}\right)$, since $\sum_{l} p_{l}=1$, so that the summation over $l$ and the logarithm can be carried out in reverse order up to corrections of the order $n^{2}$. 


\subsubsection{Saddle point approximation}

As all terms in (2.40) are extensive, the integration over the fields $\rho_{l}(\boldsymbol{x})$ and $\hat{\rho}_{l}(\boldsymbol{x})$ can be evaluated with saddle point integration, i.e. by approximating

$$
Z_{n} \approx \exp \left\{-n \mathcal{F}\left(\left\{\bar{\rho}_{l}(\boldsymbol{x}), \overline{\hat{\rho}}_{l}(\boldsymbol{x})\right\}\right)+\mathcal{O}\left(n^{2}\right)\right\}
$$

with the saddle point values $\bar{\rho}_{l}(\boldsymbol{x})$ and $\overline{\hat{\rho}}_{l}(\boldsymbol{x})$ that make $\mathcal{F}$ stationary. To eliminate the auxiliary fields $\hat{\rho}$, they are integrated out on the saddle point level,

$$
\left.\frac{\delta \mathcal{F}}{\delta \hat{\rho}_{l}(\boldsymbol{x})}\right|_{\left\{\hat{\rho}_{l}=\overline{\hat{\rho}}_{l}\right\}}=0 \quad \Rightarrow \quad \overline{\hat{\rho}}_{l}(\boldsymbol{x})=\frac{\mathrm{i}}{L} \ln \left(\frac{a_{l}}{N L p_{l}} \rho_{l}(\boldsymbol{x})\right),
$$

yielding

$$
\begin{aligned}
\mathcal{F}\left(\left\{\rho_{l}(\boldsymbol{x})\right\}\right) & :=\mathcal{F}\left(\left\{\rho_{l}(\boldsymbol{x}), \overline{\hat{\rho}}_{l}(\boldsymbol{x})\right\}\right) \\
& =\frac{1}{M} \int \mathrm{d} d x\left\{\frac{1}{Q} \sum_{l=0}^{Q} \rho_{l}(\boldsymbol{x}) \ln \rho_{l}(\boldsymbol{x})-\frac{\chi M}{4}\left(\sum_{l=0}^{Q} \mathfrak{q}_{l} \rho_{l}(\boldsymbol{x})\right)^{2}\right\},
\end{aligned}
$$

where we have omitted the additive constant $N \sum_{l=0}^{Q} p_{l} \ln \left(N L p_{l}\right)$. Thus, the disorder averaged free energy is given by

$$
F=\lim _{n \rightarrow 0} \frac{1-Z_{n}}{n}=\mathcal{F}\left(\left\{\bar{\rho}_{l}(\boldsymbol{x})\right\}\right)+\text { const.. }
$$

Within mean-field approximation, the saddle point $\{\bar{\rho}(\boldsymbol{x})\}$ is interpreted as the equilibrium value of the order parameter.

\section{Scaling with the block size}

From eq. (2.44) we can read off that changing the block size just leads to a rescaling of $\chi$ and the energy scale; hence in principle, it suffices to consider the case $M=1$.

\subsubsection{Flory-Huggins free energy}

The effective free energy (2.44) is essentially equal to that used in [15], which we shall demonstrate in the following. In ref. [15], compositions are expressed in terms of the content of $A$ monomers without counting $B$ monomers as "negative charges", so that the composition of chains of component $l$ is given by

$$
\mathfrak{c}_{l}:=l / Q=\left(\mathfrak{q}_{l}+1\right) / 2 .
$$

Thus, the local composition and its second moment at point $\boldsymbol{x}$ read

$$
\bar{c}(\boldsymbol{x}):=\sum_{l=0}^{Q} \mathfrak{c}_{l} \rho_{l}(\boldsymbol{x}) / \varrho_{0} \quad \text { and } \quad \overline{c^{2}}(\boldsymbol{x}):=\sum_{l=0}^{Q} \mathfrak{c}_{l}^{2} \rho_{l}(\boldsymbol{x}) / \varrho_{0} .
$$


Substituting $\mathfrak{q}_{l}$ into eq. (2.44) and omitting

$$
\frac{1}{M} \int \mathrm{d}^{d} x \chi M \varrho_{0} \sum_{l=0}^{Q}\left(\mathfrak{c}_{l}-\frac{1}{2}\right)^{2} \rho_{l}(\boldsymbol{c})=\chi \varrho_{0} N L \sum_{l=0}^{Q} p_{l}\left(\mathfrak{c}_{l}-\frac{1}{2}\right)^{2}
$$

as an additive constant yields

$$
\mathcal{F}\left(\left\{\rho_{l}(\boldsymbol{x})\right\}\right)=\frac{1}{M} \int \mathrm{d}^{d} x\left\{\frac{1}{Q} \sum_{l=0}^{Q} \rho_{l}(\boldsymbol{x}) \ln \rho_{l}(\boldsymbol{x})+\chi M \rho_{0}^{2}\left(\overline{c^{2}}(\boldsymbol{x})-(\bar{c}(\boldsymbol{x}))^{2}\right)\right\},
$$

which is exactly the free energy of the copolymer melt of ref. [15]. It should be noted that eq. (2.48) and thus the above derivation only hold in the incompressible limit.

\subsubsection{Multiphase equilibria}

With the effective free energy eq. (2.49) at hand, we now can study phase separated states. As pointed out in section 2.4.5, it is sufficient to discuss the case of block size one, so we let $M=1$ and $L=Q$ in the following. To consider the coexistence of $K \leq L+1$ homogeneous phases with different composition in analogy to [15], we divide the total volume $V$ into $K$ disjoint sub-volumes $V^{(k)}$ with $\sum_{k=1}^{K} V^{(k)}=V$. Each species is allowed to have a different density in each sub-volume:

$$
\rho_{l}(\boldsymbol{x})=: \rho_{l}^{(k)} \text { for } \quad \boldsymbol{x} \in V^{(k)} .
$$

In phase equilibrium, the chemical potential of a species $l$ must be the same in all phases. We define the number $N_{l}^{(k)}$ of chains of species $l$ in phase $k$ and the total number of chains in phase $k$, which is given by $N^{(k)}:=\sum_{l=1}^{L} N_{l}^{(k)}$. To compute the chemical potentials, we eliminate the densities in favour of the particle numbers,

$$
N_{l}^{(k)} / N^{(k)}=\rho_{l}^{(k)} / \varrho_{0} .
$$

Due to incompressibility,

$$
V^{(k)}=V \frac{N^{(k)}}{N}
$$

so that the free energy (2.49), up to additive constants, is given by

$$
\mathcal{F}\left(\left\{N_{l}^{(k)}\right\}\right)=\sum_{k=1}^{K} N^{(k)}\left[\sum_{l=0}^{L} \frac{N_{l}^{(k)}}{N^{(k)}} \ln \frac{N_{l}^{(k)}}{N^{(k)}}+\frac{\chi L \varrho_{0}}{4}\left(\sum_{l=0}^{L} \mathfrak{q}_{l} \frac{N_{l}^{(k)}}{N^{(k)}}\right)^{2}\right] .
$$

With the chemical potential $\mu_{l}^{(k)}$ of species $l$ in phase $k$ defined as

$$
\mu_{l}^{(k)}:=\left.\frac{\partial \mathcal{F}\left(\left\{N_{l}^{(k)}\right\}\right)}{\partial N_{l}^{(k)}}\right|_{N_{l}=\text { const. }},
$$




\section{Random copolymer melts}

the equilibrium condition reads

$$
\mu_{l}^{(k)}=\mu_{l} \quad \text { for } \quad k=1, \ldots, K,
$$

with a common chemical potential $\mu_{l}$ of species $l$ in all phases. The constraints $\sum_{i=1}^{K} N_{l}^{(k)}=$ const. in the derivative in eq. (2.54) are taken into account with the aid of Lagrange multipliers. Equating the chemical potentials according to eq. (2.55) and substituting back the particle numbers with densities, we finally arrive at

$$
\varrho_{0}=\sum_{l=0}^{L} \rho_{l}^{(k)}
$$

and

$$
\frac{\rho_{l}^{(k)}}{\varrho_{0}}=\frac{p_{l} \exp \left\{\frac{\chi \varrho_{0} L}{4}\left[2 \mathfrak{q}_{l} \sum_{l^{\prime}=0}^{L} \mathfrak{q}_{l^{\prime}} \frac{\rho_{l^{\prime}}^{(k)}}{\varrho_{0}}-\left(\sum_{l^{\prime}=0}^{L} \mathfrak{q}_{l^{\prime}} \frac{\rho_{l^{\prime}}^{(k)}}{\varrho_{0}}\right)^{2}\right]\right\}}{\sum_{k^{\prime}=1}^{K} \frac{V^{\left(k^{\prime}\right)}}{V} \exp \left\{\frac{\chi \varrho_{0} L}{4}\left[2 \mathfrak{q}_{l} \sum_{l^{\prime}=0}^{L} \mathfrak{q}_{l^{\prime}} \frac{\rho_{l^{\prime}}^{\left(k^{\prime}\right)}}{\varrho_{0}}-\left(\sum_{l^{\prime}=0}^{L} \mathfrak{q}_{l^{\prime}} \frac{\rho_{l^{\prime}}^{\left(k^{\prime}\right)}}{\varrho_{0}}\right)^{2}\right]\right\}}
$$

for $k=1, \ldots, K$. The volume fractions $\left\{V^{(k)} / V\right\}$ and the densities $\left\{\rho_{l}^{(k)}\right\}$ have to be determined self-consistently as solutions of eqs. (2.56), (2.57). The constraints

$$
\sum_{k=1}^{K} V^{(k)} \rho_{l}^{(k)}=N L \quad \text { and } \quad \sum_{k=1}^{K} V^{(k)}=V
$$

are implicitly satisfied. Equations (2.56), (2.57) are equivalent to eqs. (12), (13) of reference [15].

\section{Numerical solution}

In [15], Bauer proposed a simple algorithm, a fixed point iteration, to determine the phase equilibrium. Adapted to the present formulation (2.56), (2.57), it looks as follows:

1. Guess the number $K$ of phases; over-estimating is safe (see step 7).

2. Define an initial estimate for the volume fractions $v^{(k)}:=V^{(k)} / V$ and the average compositions (charge densities) $\sigma^{(k)}:=\sum_{l=0}^{L} \mathfrak{q}_{l} \rho_{l}^{(k)}$ of the phases.

3. Compute the partial densities $\rho_{l}^{(k)}$ according to eq. (2.57).

4. Compute $\sigma^{(k)}$ and $\rho^{(k)}:=\sum_{l=0}^{L} \rho_{l}^{(k)}$. Rescale $v^{(k)} \rightarrow v^{(k)} \cdot \rho^{(k)} / \varrho_{0}$ to enforce incompressibility (in an incompressible melt, $\rho^{(k)}$ should equal $\varrho_{0}$ ).

5. Calculate a measure for the difference between the new and the old values of $v^{(k)}$ and $\sigma^{(k)}$,e.g., the sum of the absolute values of all differences. 
6. Repeat steps $3-5$ until the discrepancy falls below a pre-defined threshold.

7. If the number of phases $K$ is overestimated, the calculation may produce phases with zero volume or multiple phases with identical composition. Delete the zero-volume phases and join the phases with equal composition.

To compute the phase diagram over an extended range of $\chi$, it turns out to be useful to count down $\chi$ from the upper to the lower limit, using the result of each iteration as the initial estimate for the next. (The reverse way is barred since the iteration fails to generate new phases from scratch; on lowering $\chi$, the number of phases decreases stepwise.) We have implemented the algorithm to compute phase equilibria; two examples are shown in fig. 2.2.

\subsubsection{Results}

The multi-component theory predicts a series of phase separations into more and more phases, which are extensively discussed in ref. [16]. Melts with symmetric and asymmetric composition behave qualitatively different. In the asymmetric case $p \neq 0$, new phases appear with zero volume and minority composition at the transition points; the transitions are of first order. Upon increasing $\chi$, they grow and achieve a more and more moderate composition. The compositions and volume fractions of the phases are shown in fig. $2.2(\mathrm{a})$ for $L=10, \lambda=0$ and $p=0.3$. In the symmetric case $p=0.5$, each phase coexists with its mirror image for reasons of symmetry. On cooling, the homogeneous phase first splits into two parts with equal volume and opposite compositions $0.5 \varrho_{0} \pm \Delta \sigma$ in a second-order transition. A third phase comes in with zero volume and symmetric composition in a first-order transition; on increasing $\chi$, this phase eventually splits into two with opposite composition and so on. The symmetric case with $L=10$ and $\lambda=0$ is shown in fig. 2.2(b).

The distribution of the charges $q_{i}$ of the chains becomes narrower with increasing chain length. As a consequence, the compositional difference of the phases and the "windows" $\Delta \chi$ between successive phase transitions become smaller. In the limit $L \rightarrow \infty$ of infinite chain length, the theory predicts the separation into arbitrary many phases with equal composition (the variance of the charge per chain goes to zero), which is obviously nonsensical. This is in agreement with the finding of ref. [18] that for chains of infinite length, macrophase separation does not occur.

\subsection{Inhomogeneous phases}

As pointed out by Fredrickson et al. [17], a random copolymer melt may exhibit, besides separation into homogeneous phases, also more exotic phases that are characterised by strongly inhomogeneous concentrations. To study such phases, concentration fluctuations on all length-scales must be taken into account, so that the crushed polymer approximation is unsuitable for this purpose. In this section 

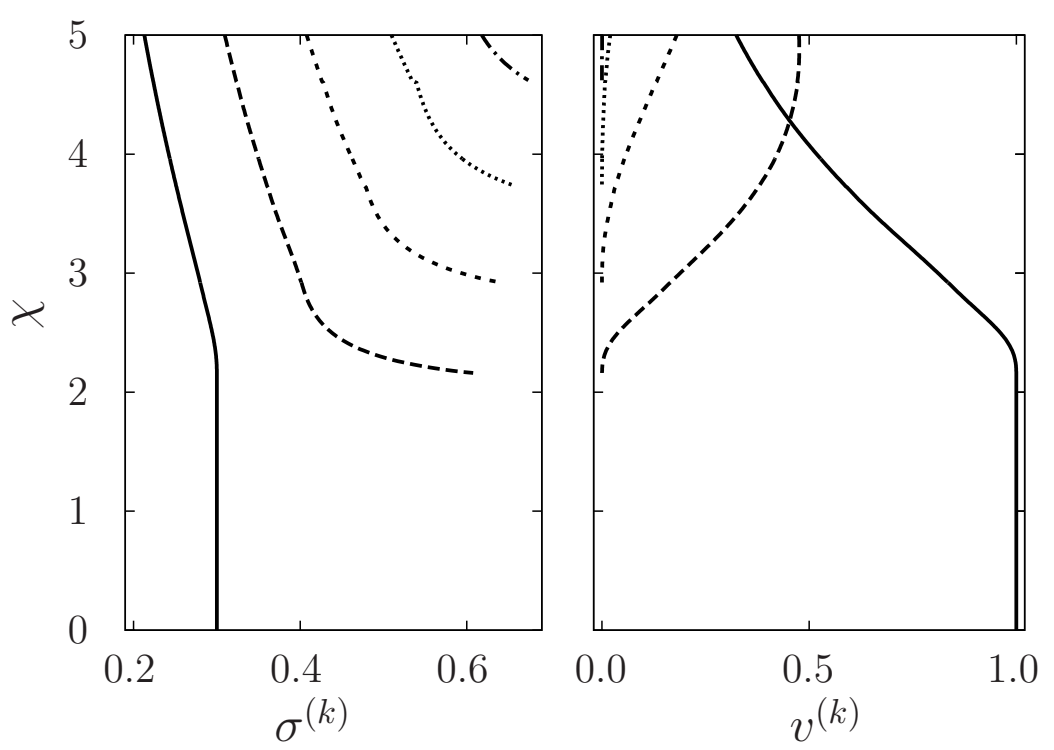

(a)
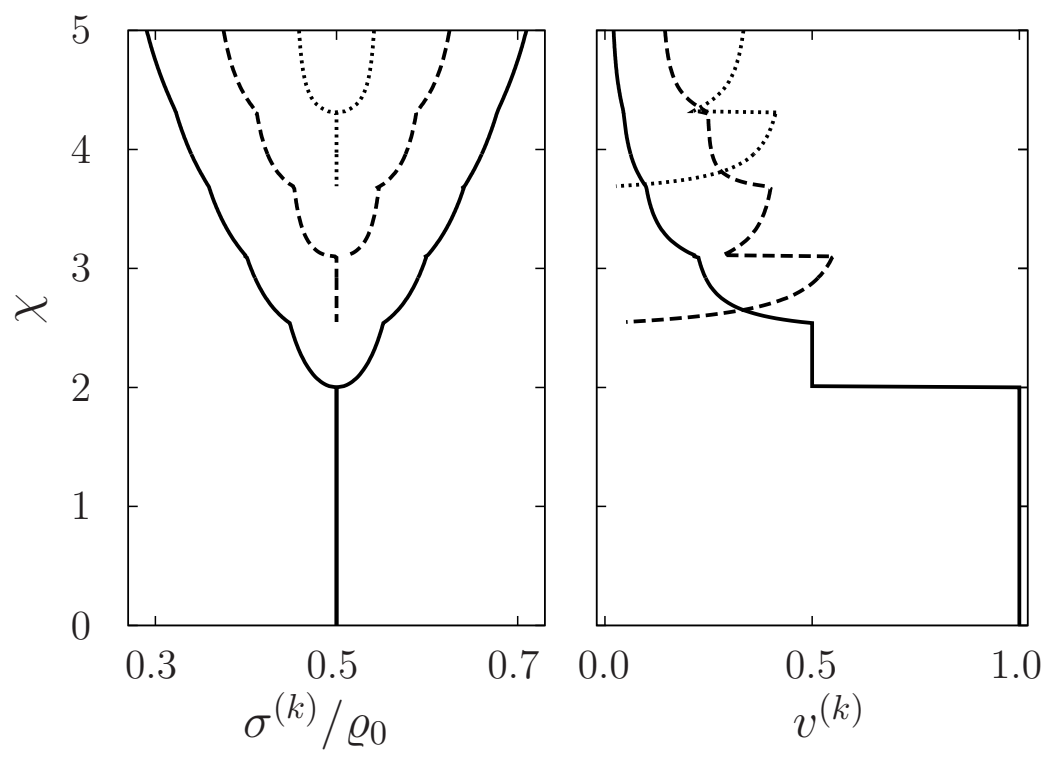

(b)

Figure 2.2.: Separation into multiple phases within Flory-Huggins theory. The lines indicate the compositions $\sigma^{(k)}$ and volume fractions $v^{(k)}$ of the phases, respectively, for $M=1, L=10$ and $\lambda=0$. The asymmetric case $p=0.3$ is shown in panel (a), the symmetric case $p=0.5$ in panel (b). 
we review the work of Fredrickson et al. [17] and extend it in several respects: We discuss compressible melts and show that compressibility favours phase separation. To include the case of short blocks and chains, our theory avoids continuum approximations, and it is shown that chains built of short blocks behave qualitatively different from chains with very large blocks. Aside from a Landau expansion, we also present the full nonlinear theory, which will be used to derive multiphase coexistence in terms of the charge density instead of the component densities of section 2.4 .

\subsubsection{Order parameters}

The impossibility of keeping track of the large number of charge and positional variables makes it desirable to introduce order parameters that disregard the conformations of individual chains. Like the pressure of a gas in a box, this would provide a more suitable description of the meso- or macroscopic state of the system. Technically, the introduction of order parameters will also help to reduce the original many-chain problem to a single chain problem. As pointed out before, the component densities of section 2.4 .2 are unsuited to describe spatially structured phases. Instead, we choose use the local monomer density $\rho(\boldsymbol{x})$ and the local imbalance of type $A$ and $B$ monomers, the charge density $\sigma(\boldsymbol{x})$, the replicated Fourier components of which are given by

$$
\rho_{\boldsymbol{k}}^{\alpha}:=\sum_{i=1}^{N} \sum_{s=1}^{L} \mathrm{e}^{\mathrm{i} \boldsymbol{k} \boldsymbol{r}_{i}^{\alpha}(s)} \quad \text { and } \quad \sigma_{\boldsymbol{k}}^{\alpha}:=\sum_{i=1}^{N} \sum_{s=1}^{L} q_{i}(s) \mathrm{e}^{\mathrm{i} \boldsymbol{k} \boldsymbol{r}_{i}^{\alpha}(s)}
$$

respectively. While the $\boldsymbol{k} \neq \mathbf{0}$ components of $\rho$ will be suppressed by imposing incompressibility, those of $\sigma$ will allow for detecting phase separation, for discriminating between macroscopic or microscopic separation, and for determining the morphology of a microphase separated state. The $\boldsymbol{k}=\mathbf{0}$ components are constant, given by the total number $N L$ of monomers and the asymmetry $q N L$ of the global composition. Note that $\rho_{\mathbf{0}}$ differs from the previously defined average density $\varrho_{0}=N L / V$ in real space by a factor of $V$.

\subsubsection{Effective free energy}

Our starting point is the replicated partition function eq. (2.23). Like in section 2.4.2, the order parameters (2.59) are introduced by integration over delta functions. With an analogous notation for the integral measures we obtain

$$
Z_{n}:=\int \mathcal{D} \rho_{\boldsymbol{k}}^{\alpha} \mathcal{D} \hat{\rho}_{\boldsymbol{k}}^{\alpha} \mathcal{D} \sigma_{\boldsymbol{k}}^{\alpha} \mathcal{D} \hat{\sigma}_{\boldsymbol{k}}^{\alpha} \exp \left(-n \mathcal{F}_{n}\left(\left\{\rho_{\boldsymbol{k}}, \hat{\rho}_{\boldsymbol{k}}, \sigma_{\boldsymbol{k}}, \hat{\sigma}_{\boldsymbol{k}}\right\}\right)\right)
$$




\section{Random copolymer melts}

with an effective free energy

$$
\begin{gathered}
n \mathcal{F}_{n}\left(\left\{\rho_{\boldsymbol{k}}, \hat{\rho}_{\boldsymbol{k}}, \sigma_{\boldsymbol{k}}, \hat{\sigma}_{\boldsymbol{k}}\right\}\right):=-\mathrm{i} \sum_{\alpha=1}^{n} \sum_{\boldsymbol{k}}^{\prime}\left(\hat{\rho}_{\boldsymbol{k}}^{\alpha} \rho_{-\boldsymbol{k}}^{\alpha}+\hat{\sigma}_{\boldsymbol{k}}^{\alpha} \sigma_{-\boldsymbol{k}}^{\alpha}\right) \\
+\frac{\mu}{4 V} \sum_{\alpha=1}^{n} \sum_{\boldsymbol{k}}^{\prime} \rho_{-\boldsymbol{k}}^{\alpha} U_{k} \rho_{\boldsymbol{k}}^{\alpha}-\frac{\chi}{4 V} \sum_{\alpha=1}^{n} \sum_{\boldsymbol{k}}^{\prime} \sigma_{-\boldsymbol{k}}^{\alpha} W_{k} \sigma_{\boldsymbol{k}}^{\alpha} \\
-N \ln \left[\left\langle\exp \left(-\mathrm{i} \sum_{\alpha=1}^{n} \sum_{\boldsymbol{k}}^{\prime} \sum_{s=1}^{L}\left(\hat{\rho}_{\boldsymbol{k}}^{\alpha}+q(s) \cdot \hat{\sigma}_{\boldsymbol{k}}^{\alpha}\right) \mathrm{e}^{-\mathrm{i} \boldsymbol{k} \boldsymbol{r}^{\alpha}(s)}\right)\right\rangle\right]+\mathcal{C}
\end{gathered}
$$

The free energy (2.61) is already expressed in the single-chain formulation, with the positional variables $\boldsymbol{r}^{\alpha}(s)$, the charges $q(s)$ and the according sequence average [... ] of the remaining chain, and with the respective Wiener average

$$
\langle\cdots\rangle:=\frac{\int \mathcal{D} \boldsymbol{r}^{\alpha}(s) \cdots \exp \left\{-\frac{1}{2} \sum_{\alpha=1}^{n} \sum_{s=1}^{L-1}\left(\boldsymbol{r}^{\alpha}(s+1)-\boldsymbol{r}^{\alpha}(s)\right)^{2}\right\}}{\int \mathcal{D} \boldsymbol{r}^{\alpha}(s) \exp \left\{-\frac{1}{2} \sum_{\alpha=1}^{n} \sum_{s=1}^{L-1}\left(\boldsymbol{r}^{\alpha}(s+1)-\boldsymbol{r}^{\alpha}(s)\right)^{2}\right\}} .
$$

The summations $\sum_{k}^{\prime}$ run over all nonzero vectors in reciprocal space, $U_{k}$ and $W_{k}$ denote the Fourier components of the (isotropic) range functions $U(r)$ and $W(r)$. The constant $\mathcal{C}$ originates from the normalisation of the Wiener average and from the $\boldsymbol{k}=\mathbf{0}$ contributions which have been omitted from the sums; it will be dropped in the following.

Because all terms in (2.61) are extensive, the partition function can be evaluated by saddle point integration, i.e.

$$
Z_{n} \approx \exp \left(-n \mathcal{F}_{n}\left(\left\{\bar{\rho}_{\boldsymbol{k}}, \overline{\hat{\rho}}_{\boldsymbol{k}}, \bar{\sigma}_{\boldsymbol{k}}, \overline{\hat{\sigma}}_{\boldsymbol{k}}\right\}\right)\right) \cdot \text { const. }
$$

Consequently, the disorder averaged free energy reads

$$
F=\lim _{n \rightarrow 0} \mathcal{F}_{n}\left(\left\{\bar{\rho}_{\boldsymbol{k}}, \overline{\hat{\rho}}_{\boldsymbol{k}}, \bar{\sigma}_{\boldsymbol{k}}, \overline{\hat{\sigma}}_{\boldsymbol{k}}\right\}\right)+\text { const. }
$$

and the saddle point values of the order parameters are interpreted as the equilibrium state of the system.

To eliminate the auxiliary fields $\hat{\rho}$ and $\hat{\sigma}$ on the saddle-point level, it turns out to be convenient to solve the saddle point equations with respect to the physical fields $\rho$ and $\sigma$ for the auxiliary fields $\hat{\rho}$ and $\hat{\sigma}$ :

$$
\frac{\partial \mathcal{F}_{n}}{\partial \rho_{\boldsymbol{k}}^{\alpha}}=\frac{\partial \mathcal{F}_{n}}{\partial \sigma_{\boldsymbol{k}}^{\alpha}}=0 \Rightarrow \hat{\rho}=-\frac{\mathrm{i} \mu}{2 V} U_{k} \rho_{\boldsymbol{k}}^{\alpha} \quad \text { and } \quad \hat{\sigma}=+\frac{\mathrm{i} \chi}{2 V} U_{k} \sigma_{\boldsymbol{k}}^{\alpha}
$$

insertion into eq. (2.61) yields

$$
\begin{aligned}
& n \mathcal{F}_{n}\left(\left\{\rho_{\boldsymbol{k}}, \sigma_{\boldsymbol{k}}\right\}\right):=-\frac{\mu}{4 V} \sum_{\alpha=1}^{n} \sum_{\boldsymbol{k}}^{\prime} \rho_{-\boldsymbol{k}}^{\alpha} U_{k} \rho_{\boldsymbol{k}}^{\alpha}+\frac{\chi}{4 V} \sum_{\alpha=1}^{n} \sum_{\boldsymbol{k}}^{\prime} \sigma_{-\boldsymbol{k}}^{\alpha} W_{k} \sigma_{\boldsymbol{k}}^{\alpha} \\
& -N \ln \left[\left\langle\exp \left(\frac{1}{2 V} \sum_{\alpha=1}^{n} \sum_{\boldsymbol{k}}^{\prime} \sum_{s=1}^{L}\left(\chi W_{k} q(s) \sigma_{\boldsymbol{k}}^{\alpha}-\mu U_{k} \rho_{\boldsymbol{k}}^{\alpha}\right) \mathrm{e}^{-\mathrm{i} \boldsymbol{k} \boldsymbol{r}^{\alpha}(s)}\right)\right\rangle\right] .
\end{aligned}
$$




\section{Rescaled fields}

In general, the complexity of the sequence statistics hampers carrying out the disorder average in the effective free energy in the exact form (2.66), thus $\mathcal{F}_{n}$ will be expanded about the incompressible, homogeneous solution $\rho_{\boldsymbol{k}}=\sigma_{\boldsymbol{k}}=0$. In this expansion, the charges appear in the form of moments $\left[q\left(s_{1}\right) q\left(s_{2}\right) \ldots q\left(s_{\nu}\right)\right]$. We replace the $\{q(s)\}$ by the centred charges, i.e. their deviation

$$
\check{q}(s):=q(s)-q
$$

from the mean charge $q$, since the moments of the latter are simpler to compute in the case of asymmetric melts. To absorb the terms in the mean charge $q$ occurring in the effective free energy, we introduce rescaled order parameter fields

$$
\Omega_{\boldsymbol{k}}^{\alpha}:=\mu U_{k} \rho_{\boldsymbol{k}}^{\alpha}-q \chi W_{k} \sigma_{\boldsymbol{k}}^{\alpha} \quad \text { and } \quad \Psi_{\boldsymbol{k}}^{\alpha}:=\chi W_{k} \sigma_{\boldsymbol{k}}^{\alpha}
$$

and obtain

$$
\begin{aligned}
n \mathcal{F}_{n}\left(\left\{\Omega_{\boldsymbol{k}}, \Psi_{\boldsymbol{k}}\right\}\right) & := \\
\frac{1}{4 V} \sum_{\alpha=1}^{n} \sum_{\boldsymbol{k}}^{\prime} & \left(\left(\frac{1}{\chi W_{k}}-\frac{q^{2}}{\mu U_{k}}\right) \Psi_{-\boldsymbol{k}}^{\alpha} \Psi_{\boldsymbol{k}}^{\alpha}-\frac{2 q}{\mu U_{k}} \Psi_{-\boldsymbol{k}}^{\alpha} \Omega_{\boldsymbol{k}}^{\alpha}-\frac{1}{\mu U_{k}} \Omega_{-\boldsymbol{k}}^{\alpha} \Omega_{\boldsymbol{k}}^{\alpha}\right) \\
& -N \ln \left[\left\langle\exp \left(\frac{1}{2 V} \sum_{\alpha=1}^{n} \sum_{\boldsymbol{k}}^{\prime} \sum_{s=1}^{L}\left(\check{q}(s) \Psi_{\boldsymbol{k}}^{\alpha}-\Omega_{\boldsymbol{k}}^{\alpha}\right) \mathrm{e}^{-\mathrm{i} \boldsymbol{k} \boldsymbol{r}^{\alpha}(s)}\right)\right\rangle\right]
\end{aligned}
$$

It might seem more natural to redefine the charge density rather than the monomer density. However, with definition (2.68) the saddle point value of the shifted density field is of the order $\mathcal{O}\left(\Psi^{2}\right)$ instead of $\mathcal{O}(\Psi)$ under certain conditions. To obtain a consistent expansion of the effective free energy to, e.g., fourth order in $\Psi$, it is then sufficient to expand to only second order in $\Omega$.

\subsubsection{Stability of the homogeneous phase}

To discuss the spinodal, i.e. the stability limit of the homogeneous phase, we expand (2.69) to second order in $\Psi$ and $\Omega$, yielding

$$
\begin{aligned}
n \mathcal{F}_{n} \approx \frac{N}{8 V^{2}} \sum_{\alpha=1}^{n} \sum_{\boldsymbol{k}}^{\prime}\{ & -\left(\frac{2 V}{N \mu U_{\boldsymbol{k}}}+D\left(\boldsymbol{k}^{2}\right)\right) \Omega_{-\boldsymbol{k}}^{\alpha} \Omega_{\boldsymbol{k}}^{\alpha}-\frac{4 q V}{N \mu U_{k}} \Omega_{-\boldsymbol{k}}^{\alpha} \Psi_{\boldsymbol{k}}^{\alpha} \\
& \left.+\left(\frac{2 V}{N \chi W_{\boldsymbol{k}}}-\frac{2 V q^{2}}{N \mu U_{k}}-\left(1-q^{2}\right) S\left(\boldsymbol{k}^{2}\right)\right) \Psi_{-\boldsymbol{k}}^{\alpha} \Psi_{\boldsymbol{k}}^{\alpha}\right\}
\end{aligned}
$$

with the discrete version of the Debye function $D\left(\boldsymbol{k}^{2}\right)$ and the charge density correlator $S\left(\boldsymbol{k}^{2}\right)$ defined in appendix C.3.1 and C.3.2. The excluded volume parameter $\mu$ is assumed to be large enough to prevent the collapse to a globular state 


\section{Random copolymer melts}

by limiting modulations of the mass density. Thus, we expect an instability of the homogeneous state to charge density fluctuations, whereas the mass density should remain noncritical. Consequently, we may integrate out the mass density fluctuations; the substitution of $\Omega$ with its saddle point value

$$
\bar{\Omega}_{\boldsymbol{k}}^{\alpha}\left(\left\{\Psi_{\boldsymbol{k}}^{\alpha}\right)\right\}=-\frac{q}{1+N \mu U_{k} D\left(\boldsymbol{k}^{2}\right) / 2 V} \Psi_{\boldsymbol{k}}^{\alpha}+\mathcal{O}\left(\Psi^{2}\right)
$$

finally yields

$$
n \mathcal{F}_{n} \approx \frac{N}{8 V^{2}} \sum_{\alpha, \boldsymbol{k}}^{\prime}\left(\frac{2 V}{N \chi W_{k}}-\left(1-q^{2}\right) S\left(\boldsymbol{k}^{2}\right)-\frac{q^{2} D\left(\boldsymbol{k}^{2}\right)}{1+N \mu U_{k} D\left(\boldsymbol{k}^{2}\right) / 2 V}\right) \Psi_{-\boldsymbol{k}}^{\alpha} \Psi_{\boldsymbol{k}}^{\alpha},
$$

with the shorthand $\sum_{\alpha, \boldsymbol{k}}^{\prime}:=\sum_{\alpha=1}^{n} \sum_{\boldsymbol{k}}^{\prime}$.

\section{Incompressible melts}

We first consider the incompressible limit $\mu \rightarrow \infty$, so that density fluctuations are suppressed. In this limit, eq. (2.72) becomes

$$
n \mathcal{F}_{n} \approx \frac{N}{8 V^{2}} \sum_{\alpha, \boldsymbol{k}}^{\prime}\left(\frac{2 V}{N \chi W_{k}}-\left(1-q^{2}\right) S\left(\boldsymbol{k}^{2}\right)\right) \Psi_{-\boldsymbol{k}}^{\alpha} \Psi_{\boldsymbol{k}}^{\alpha},
$$

with the quadratic coefficient indicating the inverse susceptibility to charge density fluctuations. The homogeneous state is stable for small $\chi$ and becomes unstable at some value $\chi_{\mathrm{s}}$ to fluctuations either at $k^{2}=0$ or at finite $k^{2}$, depending on the location of the maximum of $W_{k} \cdot S\left(k^{2}\right)$.

In the simplest case $M=1$, i.e. "no blockiness" and $Q=L$, the function $S\left(k^{2}\right)$ only depends on $x=\lambda \exp \left\{-1 /\left(2 k^{2}\right)\right\}$, cf. appendix C.3.2:

$$
\left.S\left(k^{2}\right)\right|_{M=1, Q=L}=L \frac{1+x}{1-x}-2 \frac{x\left(1-x^{L}\right)}{(1-x)^{2}} .
$$

It can be shown to increase strictly monotonically as a function of $x$ for $L \geq$ 2 (for $L=1$, it is identically equal to one). This implies a decrease with $k^{2}$ for predominantly homopolymeric chains $(\lambda>0)$ and an increase with $k^{2}$ for predominantly alternating chains $(\lambda<0)$.

For $\lambda>0$, the homogeneously mixed state becomes unstable with respect to long wavelength fluctuations first, so that the shape of the incompatibility interaction $W(r)$ can be approximated by a delta-function. For $\lambda<0$ in contrast, the instability is expected to occur at short wavelengths, so we must take into account the nonzero interaction range $\ell_{0}>0$. For $W_{k} \propto \exp \left\{-\left(k \ell_{0}\right)^{2}\right\}$, for instance, $W_{k} \cdot S\left(k^{2}\right)$ decays to zero for large $k^{2}$, so that the maximum of this expression is located at a finite value of the order $k \sim 1 / \ell_{0}$, corresponding to microscopic phase separation on length-scales of the order $\ell_{0}$. A neglect of $\ell_{0}$ would have lead to the unphysical result of phase separation on infinitely small scales in this case. 
For general block size $M$, the stability limit for charge density fluctuation modes with a particular wavenumber $k$ reads

$$
\chi_{\mathrm{s}}\left(k^{2}\right):=\frac{2}{M \varrho_{0} W_{k}} \cdot \frac{M^{2} Q}{\left(1-q^{2}\right) S\left(k^{2}\right)},
$$

hence the spinodal is given by

$$
\chi_{\mathrm{s}}:=\min \left\{\chi_{\mathrm{s}}\left(k^{2}\right)\right\}
$$

For $\lambda \geq \lambda_{\mathrm{L}}(M, Q)$, eq. (2.75) is minimised by $k=0$, so that $\chi_{\mathrm{s}}=\chi_{\mathrm{s}}(0)$. The spinodal for $\lambda<\lambda_{\mathrm{L}}$ and the threshold $\lambda_{\mathrm{L}}(M, Q)$ itself must, in general, be computed numerically. For $M=1$ we have $\lambda_{\mathrm{L}}(M=1, Q)=0$ according to the above discussion, in case of long chains it is approximately given by -0.3 .

For large block sizes $M$ and $\lambda \geq \lambda_{\mathrm{L}}$, where it is justified to take the continuum limit and let $W_{k}=1$, we recover the result of Fredrickson et al. [17] for the spinodal:

$$
M \chi_{\mathrm{s}} \varrho_{0}=\frac{2(1-\lambda)}{\left(1-q^{2}\right)(1+\lambda)\left(1-\frac{2 \lambda}{Q} \frac{1-\lambda^{Q}}{1-\lambda}\right)} \stackrel{|\lambda|^{Q} \ll 1 \ll Q}{\approx} \frac{2(1-\lambda)}{\left(1-q^{2}\right)(1+\lambda)}
$$

In the limit of large $M$ and $Q$ there are analytic expressions for $\lambda_{\mathrm{L}}=\sqrt{3}-2 \approx$ -0.27 and for the spinodal

$$
M \chi_{\mathrm{s}} \varrho_{0} \approx \frac{2(1-\lambda)^{4}-2(\lambda+2+\sqrt{3})^{2}\left(\lambda_{\mathrm{L}}-\lambda\right)^{2}}{\left(1-q^{2}\right)(1-\lambda)\left(1-\lambda^{2}\right)},
$$

in the vicinity of $\lambda_{\mathrm{L}}, c f$. eq. (3.14) of reference [17].

\section{Compressible melts}

The stability of compressible melts is determined by the full quadratic functional (2.72). Density fluctuations affect charge fluctuations only for asymmetric mixtures $(q \neq 0)$, whereas for $q=0$, charge and density fluctuations decouple on the quadratic level. In general, there are be higher-order couplings which are not considered here.

In an asymmetric melt, density fluctuations tend to destabilise the homogeneous state $\Psi=0$ and hence shift the spinodal to lower values of $\chi$, corresponding to higher temperatures, see eq. (2.72). This is intuitively clear: A compressible system can lower its energy by condensing regions of energetically favourable contacts and diluting regions of unfavourable contacts. These regions are characterised by large and small $|\Psi(\boldsymbol{x})|$, respectively. Hence for $q \neq 0$, the formation of small charge density fluctuations $\Psi_{\boldsymbol{k}}$ induces, and is faciliated by, density fluctuations $\Omega_{\boldsymbol{k}}$ according to eq. (2.71), see also the following section. 


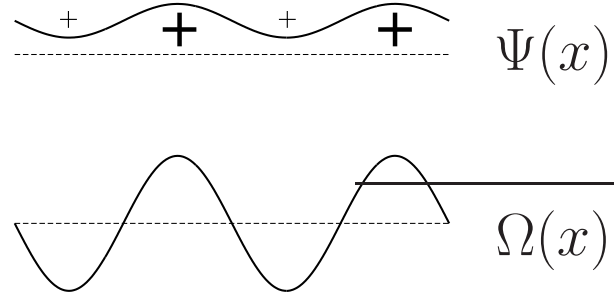

(a)
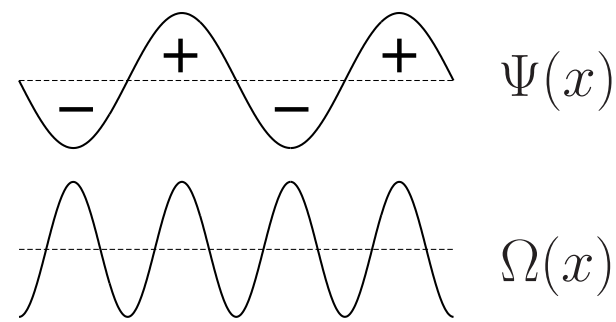

(b)

Figure 2.3.: Coupling of mass and charge density $\Omega$ and $\Psi$. Zones of high absolute charge density are condensed. For $q \neq 0$, the mass density is modulated proportional to the charge density (panel (a)). In the symmetric case, its wavelength is half as large as that of the charge density modulations (panel (b)).

\subsubsection{Landau expansion}

To address macro- and microphase separation beyond mere stability considerations, we require the Landau expansion of the free energy up to fourth order in the charge density. For small compressibility, i.e. large $\mu$, the density field turns out to be of the order $\Psi^{2}$ at the saddle point:

$$
\begin{aligned}
& \bar{\Omega}_{\boldsymbol{k}}^{\alpha}\left(\left\{\Psi_{\boldsymbol{k}}^{\alpha}\right\}\right)=\left(-\frac{q}{1+N \mu U_{\boldsymbol{k}} D\left(\boldsymbol{k}^{2}\right) / 2 V} \Psi_{\boldsymbol{k}}^{\alpha}\right. \\
& \left.+\frac{\left(1-q^{2}\right) / 4 V}{1+2 V /\left(N \mu U_{\boldsymbol{k}} D\left(\boldsymbol{k}^{2}\right)\right)} \sum_{\boldsymbol{k}^{\prime}}^{\prime} \frac{S_{2}\left(\boldsymbol{k}^{\prime}, \boldsymbol{k}-\boldsymbol{k}^{\prime}\right)}{D\left(\boldsymbol{k}^{2}\right)} \Psi_{\boldsymbol{k}^{\prime}}^{\alpha} \Psi_{\boldsymbol{k}-\boldsymbol{k}^{\prime}}^{\alpha}\right)+\mathcal{O}\left(\Psi^{3}, \frac{\Psi^{2}}{\mu^{2}}\right) \\
& \stackrel{\mu \rightarrow \infty}{\longrightarrow} \frac{1-q^{2}}{4 V} \sum_{\boldsymbol{k}^{\prime}}^{\prime} \frac{S_{2}\left(\boldsymbol{k}^{\prime}, \boldsymbol{k}-\boldsymbol{k}^{\prime}\right)}{D\left(\boldsymbol{k}^{2}\right)} \Psi_{\boldsymbol{k}^{\prime}}^{\alpha} \Psi_{\boldsymbol{k}-\boldsymbol{k}^{\prime}}^{\alpha} \quad+\mathcal{O}\left(\Psi^{3}\right)
\end{aligned}
$$

with the third order vertex function $S_{2}$ defined in appendix C.3.3. As pointed above, a compressible melt can lower its energy by diluting regions with small $|\Psi|$ and condensing those with large $|\Psi|$, corresponding to high and low densities of $A-B$ contacts, respectively. In the asymmetric case, the charge density fluctuates about $q \varrho_{0}$; w.l.o.g. we may assume $q>0$ in this paragraph. In a sinusoidal modulation of $\Psi(\boldsymbol{x})$ with small amplitude, characterised by a single wavevector $\boldsymbol{k}$, the minima and maxima correspond to small and large values of $|\Psi|$, respectively. Consequently, the induced mass density modulations follow the charge density as discussed in section 2.5.3. In a symmetric melt in contrast, the charge density fluctuates about zero mean, so that all extrema of the above sinusoidal modulation imply a high modulus $|\Psi|$. As predicated by eq. (2.79), the induced mass density modulations therefore oscillate with half the wavelength, i.e. with wavenumber $2 k$. Both cases are illustrated in fig. 2.3.

The saddle point (2.79) of $\Omega$ does not vanish for $\mu \rightarrow \infty$. This is not in contradiction with incompressibility, since the physical mass density is given by 
$\rho_{\boldsymbol{k}}=\left(\Omega_{\boldsymbol{k}}+\Psi_{\boldsymbol{k}}\right) / \mu U_{k}$ and vanishes in any case for $\mu \rightarrow \infty$. At the same time, the bulk modulus diverges, and the effect of the quadratic coupling survives the limit and contributes additional fourth order terms in $\Psi$ to the Landau expansion.

For incompressible or nearly incompressible melts, we have $\bar{\Omega}=\mathcal{O}\left(\Psi^{2}\right)$, hence the effective free energy can be expanded consistently to fourth order in $\Psi$ and just to second order in $\Omega$. Subsequently, we integrate out the density field $\Omega$ on the saddle point level according to eq. (2.79); the resulting effective free energy is given in appendix C.4. Concentrating on the incompressible limit and restricting the discussion to replica-symmetric solutions, i.e. $\Psi_{\boldsymbol{k}}^{\alpha}=\Psi_{\boldsymbol{k}}$ for $\alpha=1, \ldots, n$, we obtain

$$
\begin{aligned}
\mathcal{F}_{\text {inc }}^{\mathrm{rs}} & :=\left.\lim _{n \rightarrow 0} \mathcal{F}_{n}\right|_{\substack{\Psi_{\boldsymbol{k}}^{\alpha}=\Psi_{k} \\
\mu \rightarrow \infty}} ^{\prime} \\
= & \frac{N}{8 V^{2}} \sum_{\boldsymbol{k}}^{\prime}\left(\frac{2 V}{N \chi W_{\boldsymbol{k}}}-\left(1-q^{2}\right) S\left(\boldsymbol{k}^{2}\right)\right) \Psi_{\boldsymbol{k}} \Psi_{-\boldsymbol{k}} \\
& +\frac{N q\left(1-q^{2}\right)}{24 V^{3}} \sum_{\boldsymbol{k}_{1,2,3}}^{\prime} S_{3}\left(\boldsymbol{k}_{1}, \boldsymbol{k}_{2}\right) \delta_{\sum_{\nu=1}^{3} \boldsymbol{k}_{\nu}, \mathbf{0}} \Psi_{\boldsymbol{k}_{1}} \Psi_{\boldsymbol{k}_{2}} \Psi_{\boldsymbol{k}_{3}} \\
& +\frac{N}{128 V^{4}} \sum_{\boldsymbol{k}_{1,2,3,4}}^{\prime}\left(\left(1-q^{2}\right)^{2} \frac{S_{2}\left(\boldsymbol{k}_{1}, \boldsymbol{k}_{2}\right) S_{2}\left(\boldsymbol{k}_{3}, \boldsymbol{k}_{4}\right)}{D\left(\left(\boldsymbol{k}_{1}+\boldsymbol{k}_{2}\right)^{2}\right)}-\frac{1}{3} S_{4}\left(\boldsymbol{k}_{1}, \boldsymbol{k}_{2}, \boldsymbol{k}_{3}\right)\right) \\
& \quad \times \delta_{\sum_{\nu=1}^{4} \boldsymbol{k}_{\nu}, \mathbf{0}} \Psi_{\boldsymbol{k}_{1}} \Psi_{\boldsymbol{k}_{2}} \Psi_{\boldsymbol{k}_{3}} \Psi_{\boldsymbol{k}_{4}} \\
& +\frac{N}{128 V^{4}} \sum_{\boldsymbol{k}_{1,2}}^{\prime}\left(S_{5}\left(\boldsymbol{k}_{1}, \boldsymbol{k}_{2}\right)-\left(1-q^{2}\right)^{2} S\left(\boldsymbol{k}_{1}^{2}\right) S\left(\boldsymbol{k}_{2}^{2}\right)\right) \Psi_{\boldsymbol{k}_{1}} \Psi_{-\boldsymbol{k}_{1}} \Psi_{\boldsymbol{k}_{2}} \Psi_{-\boldsymbol{k}_{2}} \\
& +\mathcal{O}\left(\Psi^{5}\right),
\end{aligned}
$$

with the vertex functions $S_{3}, S_{4}$ and $S_{5}$ defined in appendix C.3.3. This free energy can now be probed with an ansatz for $\Psi$ corresponding to macro- or microscopic phase separation.

\section{Comparison to the free energy of Fredrickson et al.}

Fredrickson et al. apply a couple of approximations to the effective free energy:

a) They take the limit of continuous blocks and chains and expand to leading order in $1 / Q$.

b) They ignore the decay of correlations within neighbouring blocks, assuming $\boldsymbol{r}\left(s_{1}\right) \approx$ $\boldsymbol{r}\left(s_{2}\right)$ for $s_{1,2}$ residing on the same or on adjacent blocks.

c) They consider small $\lambda$ just up to linear order. 


\section{Random copolymer melts}

These approximations lead to

$$
\begin{aligned}
\mathcal{F}^{\mathrm{FML}} \approx & \frac{N M^{2} Q}{8 V^{2}} \sum_{\boldsymbol{k}}^{\prime}\left(\frac{2}{\varrho_{0} \chi M}-\left(1-q^{2}\right) s\left(M k^{2}\right)\right) \Psi_{\boldsymbol{k}} \Psi_{-\boldsymbol{k}} \\
& +\frac{N M^{3} Q q\left(1-q^{2}\right)(1+6 \lambda)}{24 V^{3}} \sum_{\boldsymbol{k}_{1,2,3}}^{\prime} \delta_{\sum_{\nu=1}^{3} \boldsymbol{k}_{\nu}, \mathbf{0}} \Psi_{\boldsymbol{k}_{1}} \Psi_{\boldsymbol{k}_{2}} \Psi_{\boldsymbol{k}_{3}} \\
+ & \frac{N M^{4} Q^{2}\left(1-q^{2}\right)^{2}(1+4 \lambda)}{64 V^{4}} \sum_{\boldsymbol{k}_{1,2}}^{\prime} g_{\mathrm{D}}\left(L\left(k_{1}^{2}+k_{2}^{2}\right)\right) \Psi_{\boldsymbol{k}_{1}} \Psi_{-\boldsymbol{k}_{1}} \Psi_{\boldsymbol{k}_{2}} \Psi_{-\boldsymbol{k}_{2}}
\end{aligned}
$$

with $W_{k}=1$ and the continuum limits $g_{\mathrm{D}}\left(L k^{2}\right) \approx D\left(k^{2}\right) / L^{2}$ and $s\left(M k^{2}\right) \approx$ $S\left(k^{2},|\lambda| \ll 1\right) / L Q$ of the second-order vertex functions defined in appendix C.3.2. The correspondence to eq. (3.2) in ref. [17] can be established via $g\left(k^{2} R_{M}^{2}\right) \leftrightarrow$ $g_{\mathrm{D}}\left(k^{2}\right)$ and $G_{2}\left(k^{2}\right) \leftrightarrow M\left(1-q^{2}\right) s\left(k^{2}\right) / 4$. As the saddle point integrations are carried out in a different order, the expansion looks slightly different in [17], but it is equivalent to eq. (2.80) for small $\left\{\Psi_{\boldsymbol{k}}\right\}$, i.e. in the range of validity, see also appendix C.6.

In the following we show that approximation (a), i.e. taking the limit $Q \rightarrow \infty$, is not entirely justified. For simplicity we consider the case of symmetric melts $(q=0)$ with uncorrelated sequences $(\lambda=0)$ and keep approximation (b), so that the cubic term vanishes and the vertex functions take a simple form. For finite $Q$ we obtain

$$
\begin{aligned}
\mathcal{F}_{\text {inc }}^{\mathrm{rs}} \approx & \frac{N M^{2} Q}{8 V^{2}} \sum_{\boldsymbol{k}}^{\prime}\left(\frac{2}{M \varrho_{0} \chi W_{k}}-\frac{D^{(M)}\left(\boldsymbol{k}^{2}\right)}{M^{2}}\right) \Psi_{\boldsymbol{k}} \Psi_{-\boldsymbol{k}} \\
& +\frac{N M^{4} Q}{192 V^{4}} \sum_{\boldsymbol{k}_{1,2,3,4}}^{\prime} \delta_{\sum_{\nu=1}^{4} \boldsymbol{k}_{\nu}, \mathbf{0}} \Psi_{\boldsymbol{k}_{1}} \Psi_{\boldsymbol{k}_{2}} \Psi_{\boldsymbol{k}_{3}} \Psi_{\boldsymbol{k}_{4}} \\
& +\frac{N M^{4} Q^{2}}{64 V^{4}} \sum_{\boldsymbol{k}_{1,2}}^{\prime}\left(\frac{D\left(k_{1}^{2}+k_{2}^{2}\right)}{M^{2} Q^{2}}-\frac{1}{Q}\right) \Psi_{\boldsymbol{k}_{1}} \Psi_{-\boldsymbol{k}_{1}} \Psi_{\boldsymbol{k}_{2}} \Psi_{-\boldsymbol{k}_{2}}
\end{aligned}
$$

with the discrete Debye function $D^{(M)}\left(k^{2}\right)$ of a single block defined in eq. (C.32). The approximation of ref. [17], by contrast, lead to

$$
\begin{aligned}
\mathcal{F}^{\mathrm{FML}} \approx & \frac{N M^{2} Q}{8 V^{2}} \sum_{\boldsymbol{k}}^{\prime}\left(\frac{2}{M \varrho_{0} \chi}-g_{\mathrm{D}}\left(M \boldsymbol{k}^{2}\right)\right) \Psi_{\boldsymbol{k}} \Psi_{-\boldsymbol{k}} \\
& +\frac{N M^{4} Q^{2}}{64 V^{4}} \sum_{\boldsymbol{k}_{1,2}}^{\prime} g_{\mathrm{D}}\left(L\left(\boldsymbol{k}_{1}^{2}+\boldsymbol{k}_{2}^{2}\right)\right) \Psi_{\boldsymbol{k}_{1}} \Psi_{-\boldsymbol{k}_{1}} \Psi_{\boldsymbol{k}_{2}} \Psi_{-\boldsymbol{k}_{2}}
\end{aligned}
$$

for $\lambda=0$ and $q=0$. This amounts to dropping the term $1 / Q$ and the whole middle term of eq. (2.82) as contributions that are formally subdominant in $Q$ compared to the remaining biquadratic term. However, the gain in free energy due to microphase separation turns out to be of the order $1 / Q$ itself. Moreover, 
the omitted middle term, corresponding to $\int d^{d} x \Psi^{4}(x)$ in space, is the only one depending on the spatial structure of a microphase separated state beyond the mere wavenumber. Therefore, besides disabling the consideration of short chains, the approximations made in [17] also lead to qualitatively different results, as will be shown in the following.

\section{Free energy of microphase separated melts}

We describe the spatial structure of the microphases with the first-harmonic ansatz suggested in ref. [17]

$$
\Psi_{\boldsymbol{k}^{\prime}}^{(m)}:=\Psi_{m} \cdot \sqrt{\frac{8}{M^{2} Q}} \cdot \frac{V}{\sqrt{2 m}} \sum_{i=1}^{m}\left(\delta_{\boldsymbol{k}^{\prime}, k \boldsymbol{n}_{i}}+\delta_{\boldsymbol{k}^{\prime},-k \boldsymbol{n}_{i}}\right),
$$

with an amplitude $\Psi_{m}$ and a wavenumber $k$ as variational parameters. The morphology is determined by lattice vectors $\left\{\boldsymbol{n}_{i}\right\}_{i=1}^{m}$. We consider three particular lattices, which are chosen because they occur in the microphase separation of regular block copolymers [3, 6]: lamellæ $(m=1)$, hexagonally arranged cylinders $(m=3)$ and spherical domains on a body centred cubic lattice $(m=6)$. These lattices are defined in appendix B.1. The real microphase morphology is, however, expected to be disordered due to the randomness of the sequences.

With the ansatz (2.84) the structure-dependent quartic term of eq. (2.82) reads

$$
\frac{N M^{4} Q}{192 V^{4}} \sum_{\boldsymbol{k}_{1,2,3,4}}^{\prime} \delta_{\sum_{\nu=1}^{4} \boldsymbol{k}_{\nu}, \mathbf{0}} \Psi_{\boldsymbol{k}_{1}}^{(m)} \Psi_{\boldsymbol{k}_{2}}^{(m)} \Psi_{\boldsymbol{k}_{3}}^{(m)} \Psi_{\boldsymbol{k}_{4}}^{(m)}=\frac{N}{3 Q} \cdot c_{m} \cdot \Psi_{m}^{4},
$$

where

$$
c_{m}:= \begin{cases}3 / 2 & \text { for } m=1 \text { (lamellæ) } \\ 5 / 2 & \text { for } m=3 \text { (cylinders) } \\ 15 / 4 & \text { for } m=6 \text { (bcc spheres) }\end{cases}
$$

see eq. (B.11). For simplicity, we take $W_{k}=1$ corresponding to $W(r)=\delta(r)$ in real space, so the Landau expansion (2.82) becomes

$$
\begin{aligned}
\mathcal{F}_{m}\left(\Psi_{m}\right) & :=\mathcal{F}\left(\left\{\Psi_{\boldsymbol{k}}^{(m)}\right\}\right) \\
& =N\left(\frac{2}{M \varrho_{0} \chi}-\frac{D^{(M)}\left(k^{2}\right)}{M^{2}}\right) \Psi_{m}^{2}+N\left(\frac{D\left(2 k^{2}\right)}{L^{2}}-\frac{1-c_{m} / 3}{Q}\right) \Psi_{m}^{4}
\end{aligned}
$$

\section{Free energy of macroscopically separated melts}

We now derive the free energy of a macroscopically separated state in terms of the Landau expansion, assuming $\lambda=0$ to simplify the vertex functions (the expressions for correlated sequences are analogous). For macroscopic phases, the dominant 
wavenumber is zero, hence we let $k=0$ in the vertex functions. With $W_{k}=1$ we obtain

$$
\begin{aligned}
& \mathcal{F}^{\text {hom }}:=N \frac{M^{2} Q}{8}(\left.\frac{2}{\varrho_{0} M \chi}-\left(1-q^{2}\right)\right) \cdot \frac{1}{V} \int \mathrm{d}^{d} x(\Psi(\boldsymbol{x}))^{2} \\
&+N \frac{M^{3} Q q\left(1-q^{2}\right)}{24} \cdot \frac{1}{V} \int \mathrm{d}^{d} x(\Psi(\boldsymbol{x}))^{3} \\
&+ N \frac{M^{4} Q^{2}}{64} \cdot \frac{\left(1-q^{2}\right)\left(1-3 q^{2}\right)}{3 Q} \cdot \frac{1}{V} \int \mathrm{d}^{d} x(\Psi(\boldsymbol{x}))^{4} \\
&+N \frac{M^{4} Q^{2}}{64}\left(\left(1-q^{2}\right)^{2}-\frac{\left(1-q^{2}\right)\left(1-3 q^{2}\right)}{Q}\right) \cdot\left(\frac{1}{V} \int \mathrm{d}^{d} x(\Psi(\boldsymbol{x}))^{2}\right)^{2} .
\end{aligned}
$$

In the simplest case, $q=0$, the volume will split into two parts $V_{1}$ and $V_{2}$ of equal size and opposite composition due to symmetry,

$$
\Psi(\boldsymbol{x})= \begin{cases}+\sqrt{\frac{8}{M^{2} Q}} \Psi_{\mathrm{hom}} & \text { for } \boldsymbol{x} \in V_{1} \\ -\sqrt{\frac{8}{M^{2} Q}} \Psi_{\mathrm{hom}} & \text { for } \boldsymbol{x} \in V_{2}\end{cases}
$$

The arbitrary factor $\sqrt{8 / M^{2} Q}$ is introduced to simplify the expression

$$
\mathcal{F}^{\text {hom }}=N\left(\frac{2}{\varrho_{0} M \chi}-1\right) \Psi_{\text {hom }}^{2}+\frac{M^{4} Q^{2}}{128}\left(1-\frac{1-1 / 3}{Q}\right) \Psi_{\text {hom }}^{4}
$$

which can formally be treated as the case " $m=0$ " of the microphase free energy (2.87) with $c_{0}=1$ and $k=0$.

\subsubsection{Microphase separation}

The microphase free energy eq. (2.87) bears the following problem: For large $k$, the quartic coefficient becomes negative, because $D\left(k^{2}\right)$ decays to zero for large argument. Fredrickson et al. ignore the term $\left(1-c_{m} / 3\right) / Q$, so that their coefficient remains nonnegative. An obvious way of removing the ill-definedness is to drop the $k$ dependence of the quartic term. This is in the spirit of the usual Ginzburg Landau expansion, which keeps terms like $(\nabla \Psi)^{2}$ but no space dependence on the quartic level. Yet, in this approximation, the homogeneously phase separated state would always be lower in energy than the microphase separated one, since $c_{m}>c_{0}=1$ for $m \in\{1,3,6\}$ and since $D^{(M)}\left(k^{2}\right)$ decreases with $k^{2}$. If at all, microphases set in with nonzero $k$.

To discuss microphase separation, we are thus forced to keep the $k$-dependence of the quartic term. We ensure its positivity by introducing a cutoff for large $k$ of the order of the inverse radius of gyration $1 / R_{M}=1 / \sqrt{M}$, avoiding the range where the coefficient falls below zero. The cutoff is well justified, since local demixing on length-scales smaller than the extension of a single block is physically impossible. 
At a given wavenumber $k$, the amplitude minimising the free energy acquires a nonzero value

$$
\Psi_{m}^{2}=\frac{-\left(\frac{2}{M \varrho 0 \chi}-\frac{D^{(M)}\left(k^{2}\right)}{M^{2}}\right)}{2\left(\frac{D\left(2 k^{2}\right)}{L^{2}}-\frac{1-c_{m} / 3}{Q}\right)}
$$

for $\chi$ exceeding the stability limit $\chi_{\mathrm{s}}\left(k^{2}\right)=2 M / \varrho_{0} D^{(M)}\left(k^{2}\right)$. In this case, eq. (2.87) becomes

$$
\mathcal{F}_{m}\left(k^{2}\right)=-\frac{N}{4} \frac{\left(\frac{2}{M \varrho_{0} \chi}-\frac{D^{(M)}\left(k^{2}\right)}{M^{2}}\right)^{2}}{\left(\frac{D\left(2 k^{2}\right)}{L^{2}}-\frac{1-c_{m} / 3}{Q}\right)} .
$$

For $k$ below the cutoff, the denominator is positive, hence this expression is minimal for the smallest $c_{m}$. Out of the considered microphase structures, the lamellar morphology yields the lowest free energy.

\section{Separation on large length-scales}

Before minimising with respect to the wavenumber, we consider the case $k=0$, which includes the possibility of macroscopic phase separation with $c_{0}=1$. As discussed earlier, the difference in the free energies of a micro- and a macrophase separated state is always positive for $k \rightarrow 0$. It is given to leading order in $1 / Q$ by

$$
\Delta \mathcal{F}_{m}(0):=\mathcal{F}_{m}(0)-\mathcal{F}_{0}(0)=\frac{N}{4}\left(\frac{\chi_{\mathrm{s}}-\chi}{\chi}\right)^{2} \frac{c_{m}-1}{Q}>0
$$

As anticipated, this difference is lost when applying the approximations made in eq. (2.81) as anticipated.

For nonzero $k$, macrophase separation is excluded, thus the smallest $c_{m}$ corresponds to the lamellar structure and the hexagonal and the cubic lattice are always unfavourable. In the following, we can therefore assume $c_{m}<3$.

\section{Small blocks (block size one)}

If the "blocks" just consist of a single monomer, i.e. $M=1$, the free energy (2.92) of the phase separated states reads

$$
\mathcal{F}_{m}\left(k^{2}\right)=\frac{N}{4} \frac{\left(1-\frac{\chi_{\mathrm{s}}}{\chi}\right)^{2}}{4\left(\frac{D\left(2 k^{2}\right)}{L^{2}}-\frac{1-c_{m} / 3}{L}\right)} .
$$

with $\chi_{\mathrm{s}}=\frac{2}{M \varrho_{0}}$. Since the numerator is independent of $k$, the free energy of the microscopically separated state is always lowered by increasing $k^{2}$ (up to the cutoff), which compensates for the disadvantage of requiring $c_{m}>c_{0}=1$. Hence, microphase separation is favoured for any $\chi>\chi_{\mathrm{s}}$. The optimal wavenumber $k_{\mathrm{opt}}$ is nonzero and independent of $\chi$, given by the cutoff introduced above. 


\section{Larger blocks}

If $M>1$, the numerator is no longer independent of $k$. The typical scale of $D^{(M)}\left(k^{2}\right)$ is $k \sim R_{M}^{-1}=1 / M$. We expect microphase separation to occur at very small $k$ at its onset and expand $D^{(M)}\left(k^{2}\right)$ for small argument up to quadratic order, as was done in ref. [17], as well.

In the case $\lambda=0$ under consideration, the spinodal reads $\chi_{\mathrm{s}}=2 / M \varrho_{0}$, see also section 2.5.3. We introduce the dimensionless squared wavenumber $x:=k^{2} R_{M}^{2}=$ $M k^{2}$ and minimise the free energy per chain,

$$
f(x):=\frac{\mathcal{F}_{m}}{N}=\frac{1}{4}\left(\frac{D^{(M)}(x / M)}{M^{2}}-\frac{\chi_{\mathrm{s}}}{\chi}\right)^{2} /\left(\frac{D(Q x / L)}{L^{2}}-\frac{1-c_{m} / 3}{Q}\right),
$$

in a quadratic expansion with respect to $x$ to find the optimal domain size of the microphase separated state. The optimal wavenumber $k_{\text {opt }}$ becomes nonzero for $\chi>\chi_{0}$ with

$$
\begin{aligned}
\chi_{0} / \chi_{\mathrm{s}} & =\left(1-\frac{\left(M^{2}-1\right)\left(Q-\left(1-c_{m} / 3\right)\right)}{L^{2}-1}\right)^{-1} \\
& =1+\frac{M^{2}-1}{M^{2}} Q^{-1}+\frac{\left(M^{2}-1\right)\left(M^{2} c_{m} / 3-1\right)}{M^{4}} Q^{-2}+\mathcal{O}\left(Q^{-3}\right) .
\end{aligned}
$$

Below this threshold, $k_{\mathrm{opt}}=0$, and thus the competing macrophase separated state is more favourable (see above). As pointed out before, microphases either set in with finite wavenumber or not at all.

To determine the point $\chi_{\mathrm{m}}$ where microphase separation with optimal wavenumber $k_{\mathrm{opt}}=: x_{\mathrm{opt}} / M$ becomes energetically equivalent to macroscopic segregation, we solve $f^{\prime}\left(x_{\mathrm{opt}}\right)=0$ for $x_{\mathrm{opt}}$. Within an expansion to linear order in $\left(\chi-\chi_{0}\right) / \chi$, we determine the zero of the excess free energy of the microphase separated state,

$$
\Delta \mathcal{F}_{m}\left(k_{\text {opt }}^{2}\right):=\mathcal{F}_{m}\left(k_{\text {opt }}^{2}\right)-\mathcal{F}_{0}(0)
$$

Microphases and homogeneous phases become energetically equivalent at $\chi=\chi_{\mathrm{m}}$ with

$$
\begin{aligned}
\chi_{\mathrm{m}} / \chi_{\mathrm{s}}=1+\frac{M^{2}-1}{M^{2}} & Q^{-1}+\frac{M^{2}-1}{M^{2}} \sqrt{\frac{2}{3}\left(c_{m}-1\right)} Q^{-3 / 2} \\
& +\frac{\left(c_{m}-\frac{2}{3}\right) M^{4}-\left(c_{m}+1\right) M^{2}+1}{M^{4}} Q^{-2}+\mathcal{O}\left(Q^{-5 / 2}\right)
\end{aligned}
$$

for $M \geq 2$, the lamellar morphology yields the lowest $\chi_{\mathrm{m}}$. The result $\chi_{\mathrm{m}} / \chi_{\mathrm{s}}-1=$ $1 / Q$ of ref. [17] is recovered to leading order in $1 / Q$ in the limit of continuous blocks (large $M$ ).

The optimal wavenumber $k_{\mathrm{opt}}=\sqrt{x_{\mathrm{opt}} / M}$ at $\chi=\chi_{\mathrm{m}}$ is given by

$$
x_{\mathrm{opt}}\left(\chi=\chi_{\mathrm{m}}\right)=\sqrt{\frac{3}{2}\left(c_{m}-1\right)} Q^{-3 / 2}+\left(c_{m}-1\right) Q^{-2}+\mathcal{O}\left(Q^{-5 / 2}\right) .
$$


Here, the result $x=0$ of ref. [17] can be recovered in the limit $Q \rightarrow \infty$, yet this implies dropping the leading order term.

For long chains, i.e. $Q \gg 1$, the free energy difference between micro- and macrophases grows roughly proportional to $\left(\chi-\chi_{\mathrm{m}}\right)^{2}$ on further increase of $\chi$ :

$$
\begin{aligned}
\Delta \mathcal{F}_{m}\left(k_{\mathrm{opt}}^{2}\right)=- & \frac{N}{4}\left(1-\frac{M^{2}\left(1+18 c_{m}\right)-48}{12 M^{2}} Q^{-1}\right)\left(\frac{\chi-\chi_{\mathrm{m}}}{\chi_{\mathrm{s}}}\right)^{2} \\
& -N \sqrt{\frac{\left(c_{m}-1\right)\left(M^{2}-1\right)^{2}}{6 M^{4}}} Q^{-3 / 2}\left(\frac{\chi-\chi_{\mathrm{m}}}{\chi_{\mathrm{s}}}\right)+\mathcal{O}\left(Q^{-2}\right) .
\end{aligned}
$$

\section{Numerical results}

We now provide some examples to illustrate the calculation. For different $Q$ and $M$ we have calculated the inverse temperatures $\chi_{0}$, at which the optimal wavenumber of lamellar microphases becomes nonzero, and $\chi_{\mathrm{m}}$, at which the lamellæ become energetically equivalent to the macroscopically separated state. While $\chi_{0}$ can be computed analytically according to eq. (2.96), the point $\chi_{\mathrm{m}}$ and the optimal wavenumber at $\chi_{\mathrm{m}}$ have been determined numerically using the fourth order Landau free energies of the macrophase separated and the lamellar microphase separated state. These data are shown in tables 2.1, 2.2 and 2.3.

For $M=1$, the numerical solution predicts microphase separation with $k=$ $\infty$, or zero wavelength, at $\chi_{\mathrm{m}}=\chi_{\mathrm{s}}$, because the cutoff $\ell_{0}$ for $k$ has not been implemented therein. The correct value would depend on $\ell_{0}$.

The case $Q=2$ is somewhat special, as well: in this case, the melt can be conceived as a symmetric blend of homopolymers and diblock copolymers without any compositional disorder. The diblocks and homopolymers can arrange in wellordered layers with a wide range of possible spacings. Moreover, the distance of $\chi_{\mathrm{m}}$ to the spinodal $\chi_{\mathrm{s}}$ is roughly proportional to $1 / Q$, which is relatively large for $Q=2$. In this range, the fourth order Landau expansion used in the numerical procedure probably is no longer valid.

For longer chains of larger blocks, i.e. for $M>1$ and $Q \geq 3$, the values of $\chi_{0}, \chi_{\mathrm{m}}$ and $x_{\mathrm{opt}}$ indeed follow the analytic predictions of eqs. (2.96), (2.98) and (2.99) for $M \rightarrow \infty$, with an accuracy increasing with $Q$, as is to be expected. For $Q \geq 5$, the ratio $\chi_{\mathrm{m}} / \chi_{\mathrm{s}}$ of the microphase transition point and the spinodal, and the lattice constant $k / R_{M}$ of the microphases in terms of the radius of gyration of the blocks depend only weakly on the block size.

\subsubsection{Flory-Huggins theory for the charge density}

In the following, we shall establish the link between the multi-component theory of section 2.4 and the Landau theory of the present section. To this end it will be shown that the full microscopic theory in terms of the charge and mass density includes the Flory-Huggins theory of multiphase coexistence. The starting point is 


\begin{tabular}{llllll} 
& $Q=2$ & $Q=3$ & $Q=5$ & $Q=10$ & $Q=20$ \\
\hline$M=1$ & 1 & 1 & 1 & 1 & 1 \\
$M=2$ & 1.429 & 1.273 & 1.157 & 1.077 & 1.038 \\
$M=5$ & 1.571 & 1.366 & 1.209 & 1.100 & 1.049 \\
$M=10$ & & 1.380 & 1.217 & 1.104 & 1.051 \\
$M=20$ & & 1.383 & 1.219 & 1.105 & 1.051 \\
Analytic theory: & & & & \\
$M \rightarrow \infty$ & 1.600 & 1.385 & 1.22 & 1.105 & 1.051
\end{tabular}

Table 2.1.: Onset $\chi_{0} / \chi_{\mathrm{s}}$ of nonzero $k$ in lamellar microphases for finite $M$ and for $M \rightarrow \infty$, computed according to eq. (2.96)

\begin{tabular}{llllll} 
& $Q=2$ & $Q=3$ & $Q=5$ & $Q=10$ & $Q=20$ \\
\hline$M=1$ & 1 & 1 & 1 & 1 & 1 \\
$M=2$ & 1.656 & 1.391 & 1.208 & 1.093 & 1.043 \\
$M=5$ & 1.854 & 1.534 & 1.279 & 1.122 & 1.056 \\
$M=10$ & & 1.556 & 1.290 & 1.126 & 1.058 \\
$M=20$ & & 1.561 & 1.292 & 1.127 & 1.058
\end{tabular}

Analytic approximation:

$M \rightarrow \infty \quad 1.912 \quad 1.537 \quad 1.285 \quad 1.127 \quad 1.059$

Table 2.2.: Onset $\chi_{\mathrm{m}} / \chi_{\mathrm{s}}$ of lamellar microphases determined numerically for finite $M$. The analytic approximation for $M \rightarrow \infty$ is computed according to eq. (2.98).

\begin{tabular}{llllll} 
& $Q=2$ & $Q=3$ & $Q=5$ & $Q=10$ & $Q=20$ \\
\hline$M=1$ & $\infty$ & $\infty$ & $\infty$ & $\infty$ & $\infty$ \\
$M=2$ & 0.59 & 0.250 & 0.099 & 0.031 & 0.011 \\
$M=5$ & 0.75 & 0.253 & 0.099 & 0.031 & 0.011 \\
$M=10$ & & 0.254 & 0.099 & 0.031 & 0.011 \\
$M=20$ & & 0.255 & 0.099 & 0.031 & 0.011
\end{tabular}

Analytic approximation:

$$
M \rightarrow \infty \quad 0.431 \quad 0.222 \quad 0.0975 \quad 0.0324 \quad 0.0109
$$

Table 2.3.: Wavenumber square $x=k^{2} R_{M}^{2}$ at $\chi=\chi_{\mathrm{m}}$ determined numerically for finite $M$. The analytic approximation for $M \rightarrow \infty$ is computed according to eq. (2.99). 
the effective free energy (2.66), which is evaluated in the crushed polymer approximation, i.e. for $\boldsymbol{r}^{\alpha}(s)=\boldsymbol{r}^{\alpha}$. Focusing on macroscopic phases, we let $W(r)=\delta(r)$ and $U(r)=\delta(r)$ and obtain

$$
\begin{aligned}
& n \mathcal{F}_{n}\left(\left\{\rho^{\alpha}, \sigma^{\alpha}\right\}\right)=-\frac{\mu}{4} \sum_{\alpha=1}^{n} \int \mathrm{d}^{d} x\left(\rho^{\alpha}(\boldsymbol{x})\right)^{2}+\frac{\chi}{4} \sum_{\alpha=1}^{n} \int \mathrm{d}^{d} x\left(\sigma^{\alpha}(\boldsymbol{x})\right)^{2} \\
& \quad-N \ln \sum_{l=0}^{Q} p_{l} \int \frac{\mathrm{d}^{n \cdot d} r}{V^{n}} \exp \left(-\frac{L \mu}{2} \sum_{\alpha=1}^{n} \rho^{\alpha}\left(\boldsymbol{r}^{\alpha}\right)+\frac{L \chi \mathfrak{q}_{l}}{2} \sum_{\alpha=1}^{n} \sigma^{\alpha}\left(\boldsymbol{r}^{\alpha}\right)\right)
\end{aligned}
$$

in the real space representation, where $\mathfrak{q}_{l}=(2 l / Q-1)$ and $p_{l}$ denote the component charges and fractions of section 2.4 .2 and 2.4.3. The expression (2.101) is further simplified with a replica-symmetric ansatz $\rho^{\alpha}(\boldsymbol{x})=\rho(\boldsymbol{x})$ and $\sigma^{\alpha}(\boldsymbol{x})=\sigma(\boldsymbol{x})$. In anticipation of the replica limit $n \rightarrow 0$, the summation over $l$ and the logarithm may be carried out in reverse order ( $c f$. section 2.4.4), yielding

$$
\mathcal{F}:=\lim _{n \rightarrow 0} \mathcal{F}_{n}:=-\frac{\mu}{4} \int \mathrm{d}^{d} x(\rho(\boldsymbol{x}))^{2}+\frac{\chi}{4} \int \mathrm{d}^{d} x(\sigma(\boldsymbol{x}))^{2}-N \sum_{l=0}^{Q} p_{l} \ln a_{l},
$$

where

$$
a_{l}:=\frac{1}{V} \int \mathrm{d}^{d} x \exp \left(-\frac{L \mu}{2} \rho(\boldsymbol{r})+\frac{L \chi \mathfrak{q}_{l}}{2} \sigma(\boldsymbol{r})\right) .
$$

\section{Multiphase equilibria}

Like in section 2.4.7, to study the separation into multiple homogeneous phases, we split the volume into $K \leq Q+1$ parts $V^{(k)}$, in which the density and the charge density may take different values

$$
\left.\begin{array}{l}
\rho(\boldsymbol{x})=: \rho^{(k)} \\
\sigma(\boldsymbol{x})=: \sigma^{(k)}
\end{array}\right\} \quad \text { for } \quad \boldsymbol{x} \in V^{(k)}
$$

Due to the conservation of the volume, the particle number and the charge, we have

$$
\sum_{k=1}^{K} v^{(k)}=1, \quad \sum_{k=1}^{K} v^{(k)} \rho^{(k)}=\varrho_{0}=\frac{N L}{V} \quad \text { and } \quad \sum_{k=1}^{K} v^{(k)} \sigma^{(k)}=q \varrho_{0},
$$

where $v^{(k)}:=V^{(k)} / V$ denote the $K$ volume fractions.

The effective free energy (2.102) with the above ansatz must be minimised with respect to $\left\{\rho^{(k)}\right\},\left\{\sigma^{(k)}\right\}$ and $v^{(k)}$; the constraints (2.105) are taken into account by means of Lagrange multipliers. This leads to the equations

$$
\begin{aligned}
\rho^{(k)} & =\varrho_{0} \sum_{l=0}^{Q} \frac{p_{l}}{a_{l}} \exp \left\{-\frac{\mu L}{2} \rho^{(k)}+\frac{\chi L \mathfrak{q}_{l}}{2} \sigma^{(k)}\right\}, \\
\sigma^{(k)} & =\varrho_{0} \sum_{l=0}^{Q} \frac{p_{l}}{a_{l}} \mathfrak{q}_{l} \exp \left\{-\frac{\mu L}{2} \rho^{(k)}+\frac{\chi L \mathfrak{q}_{l}}{2} \sigma^{(k)}\right\},
\end{aligned}
$$


and

$$
\rho^{(k)}=\Lambda-\frac{\mu L}{4}\left(\rho^{(k)}\right)^{2}+\frac{\chi L}{4}\left(\sigma^{(k)}\right)^{2}
$$

with $a_{l}$ given by

$$
a_{l}=\sum_{k=1}^{K} v^{(k)} \exp \left\{-\frac{L \mu}{2} \rho^{(k)}+\frac{L \chi \mathfrak{q}_{l}}{2} \sigma^{(k)}\right\} .
$$

One of the three Lagrange multipliers, $\Lambda$, cannot be eliminated immediately and must be determined from eqs. (2.106-2.108), as well.

In eqs. (2.106-2.109), the block size $M$ merely occurs as a pre-factor of $\mu$ and $\chi$ and can be absorbed into rescaled parameters ( $c f$. section 2.4.5), so it suffices to consider just the case $M=1$ and $Q=L$.

\section{Weakly compressible melts}

For low compressibility, i.e. for $\mu \gg 1$, the particle density can be expanded in powers of $1 / \mu$,

$$
\rho^{(k)}=\varrho_{0}+C^{(k)} \mu^{-1}+\mathcal{O}\left(\mu^{-2}\right) .
$$

With eqs. (2.110) and (2.108) it follows that

$$
\rho^{(k)}-\rho^{\left(k^{\prime}\right)}=\frac{\chi}{2 \varrho_{0}}\left(\left(\sigma^{(k)}\right)^{2}-\left(\sigma^{\left(k^{\prime}\right)}\right)^{2}\right) \cdot \mu^{-1}+\mathcal{O}\left(\mu^{-2}\right),
$$

i.e. the density variations are asymptotically proportional to the square of the variation of the charge density, like in Landau theory. The insertion of eq. (2.111) into equations (2.106), (2.107) yields

$$
\begin{aligned}
\rho^{(k)} & =\varrho_{0} \sum_{l=0}^{Q} \frac{p_{l}}{a_{l}^{\prime}} \exp \left\{\frac{\chi L}{4 \varrho_{0}}\left(2 \varrho_{0} \mathfrak{q}_{l} \sigma^{(k)}-\left(\sigma^{(k)}\right)^{2}\right)\right\}+\mathcal{O}\left(\mu^{-1}\right) \\
\sigma^{(k)} & =\varrho_{0} \sum_{l=0}^{Q} \mathfrak{q}_{l} \frac{p_{l}}{a_{l}^{\prime}} \exp \left\{\frac{\chi L}{4 \varrho_{0}}\left(2 \varrho_{0} \mathfrak{q}_{l} \sigma^{(k)}-\left(\sigma^{(k)}\right)^{2}\right)\right\}+\mathcal{O}\left(\mu^{-1}\right),
\end{aligned}
$$

where

$$
a_{l}^{\prime}=\sum_{k=1}^{K} v^{(k)} \exp \left\{\frac{\chi L}{4 \varrho_{0}}\left(2 \varrho_{0} \mathfrak{q}_{l} \sigma^{(k)}-\left(\sigma^{(k)}\right)^{2}\right)\right\} \text {. }
$$




\section{Incompressible limit}

In the incompressible limit, the terms of order $1 / \mu$ vanish, and the equations (2.112), (2.113) reduce to

$$
\begin{aligned}
\varrho_{0}=\rho^{(k)} & =\varrho_{0} \sum_{l=0}^{Q} \frac{p_{l}}{a_{l}^{\prime}} \exp \left\{\frac{\chi L}{4 \varrho_{0}}\left(2 \varrho_{0} \mathfrak{q}_{l} \sigma^{(k)}-\left(\sigma^{(k)}\right)^{2}\right)\right\} \\
\sigma^{(k)} & =\varrho_{0} \sum_{l=0}^{Q} \mathfrak{q}_{l} \frac{p_{l}}{a_{l}^{\prime}} \exp \left\{\frac{\chi L}{4 \varrho_{0}}\left(2 \varrho_{0} \mathfrak{q}_{l} \sigma^{(k)}-\left(\sigma^{(k)}\right)^{2}\right)\right\}
\end{aligned}
$$

Although $\rho^{(k)}=\varrho_{0}$ for all $k$ in the incompressible limit, the $K$ equations $(2.115)$ are required to determine the $K$ volume fractions $\left\{v^{(k)}\right\}$, which are hidden in the $\left\{a_{l}^{\prime}\right\}$ and which define the state of the system together with the charge densities. The system of equations (2.115), (2.116) is equivalent to eqs. (2.56), (2.57) of the multicomponent theory discussed in section 2.4 after summation over the components according to

$$
\rho^{(k)}:=\sum_{l=0}^{Q} \rho_{l}^{(k)} \quad \text { and } \quad \sigma^{(k)}:=\sum_{l=0}^{Q} \mathfrak{q}_{l} \rho_{l}^{(k)} .
$$

\section{Numerical solution}

The fixed point iteration for the multi-component theory of section 2.4 .7 can be easily adapted to the current formulation (2.115), (2.116):

1. Guess the number $K$ of phases; over-estimating is safe (see step 5).

2. Define an initial estimate for the volume fractions $v^{(k)}$ and the compositions $\sigma^{(k)}$ of the phases.

3. Compute $\rho^{(k)}$ and $\sigma^{(k)}$ according to eqs. (2.115) and (2.116). To enforce incompressibility, rescale $v^{(k)} \rightarrow v^{(k)} \cdot \rho^{(k)} / \varrho_{0}$.

4. Measure the difference between the old and new values of $v^{(k)}$ and $\sigma^{(k)}, e . g$. , the sum of the absolute values of all differences.

5. Drop phases with zero volume and join phases with identical composition

6. Repeat steps $3-5$ until the discrepancy falls below a pre-defined threshold.

Again, to compute the phase diagram over an extended range of $\chi$, it is useful to count down $\chi$ from the upper to the lower limit, using the result of each iteration as the initial estimate for the next. To save computer time it is advisable to compute the component-resolved densities

$$
\rho_{l}^{(k)}:=\varrho_{0} p_{l}\left(\sum_{k^{\prime}=1}^{K} v^{\left(k^{\prime}\right)} \exp \left\{\frac{\chi L}{4 \varrho_{0}}\left(\sigma^{(k)}-\sigma^{\left(k^{\prime}\right)}\right)\left(\sigma^{(k)}+\sigma^{\left(k^{\prime}\right)}-2 \mathfrak{q}_{l} \varrho_{0}\right)\right\}\right)^{-1}
$$


as intermediate results, since they appear in $\rho^{(k)}$ as well as in $\sigma^{(k)}$. The physical interpretation of eq. (2.118), however, becomes evident only in the multi-component picture of section 2.4.7.

\section{Symmetric melts}

In a symmetric blend, each phase coexists with its "mirror image" of equal size, in which $A$ and $B$ blocks are exchanged. To discuss the transition from one to two homogeneous phases we can therefore assume $\sigma^{(1)}=-\sigma^{(2)}=$ : $\sigma$ and $v^{(1)}=$ $v^{(2)}=1 / 2$. With this ansatz, the equations (2.115) are identically satisfied; the two equations (2.115) are equivalent and yield

$$
\frac{\sigma}{\varrho_{0}}=\sum_{l=0}^{(Q-1) / 2} 2 p_{l} \frac{1+2 l}{Q} \tanh \left\{\frac{M \chi}{2}(1+2 l) \sigma\right\}
$$

for odd $Q$, and

$$
\frac{\sigma}{\varrho_{0}}=\sum_{l=1}^{Q / 2} 2 p_{l} \frac{2 l}{Q} \tanh \left\{\frac{M \chi}{2}(2 l) \sigma\right\}
$$

for even $Q$.

In the case of homopolymer blends $(Q=1)$ and diblock copolymers $(Q=2)$, we obtain

$$
\frac{\sigma}{\varrho_{0}}=\tanh (M \chi \cdot \sigma / 2) \quad \text { and } \quad \frac{2 \sigma}{(1+\lambda) \varrho_{0}}=\tanh (M \chi \cdot \sigma),
$$

respectively. Obviously the case $Q=2$ can be obtained from the case $Q=1$ with the substitutions $\sigma \rightarrow 2 \sigma /(1+\lambda)$ and $\chi \rightarrow(1+\lambda) \chi$, which is intuitively clear: The neutral $A B$ diblocks are uniformly distributed and just dilute the homopolymer blend.

\subsubsection{Macrophase separation: Flory-Huggins and Landau theory}

In order to compare the predictions of the multi-component theory and the Landau theory for macroscopic separation into two phases, we extend the respective treatment of section 2.5.4 to asymmetric blends, sticking to $\lambda=0$ for the sake of simplicity. To faciliate the physical interpretation we substitute back the rescaled field $\Psi_{\boldsymbol{k}}=\chi \sigma_{\boldsymbol{k}}$. The Fourier components indicate only the deviation from the mean value, thus the corresponding relation in real space reads

$$
\tilde{\sigma}(\boldsymbol{x}):=\sigma(\boldsymbol{x})-q \varrho_{0}=\frac{1}{\chi} \Psi(\boldsymbol{x}) .
$$


In terms of $\tilde{\sigma}$, the effective free energy (2.88) for macroscopic phase separation is given by

$$
\begin{aligned}
F \cdot \frac{8}{N M^{2} Q \chi^{2}}= & a \cdot \frac{1}{V} \int \mathrm{d}^{d} x(\tilde{\sigma}(\boldsymbol{x}))^{2}+b \cdot \frac{1}{V} \int \mathrm{d}^{d} x(\tilde{\sigma}(\boldsymbol{x}))^{3} \\
& +c \cdot \frac{1}{V} \int \mathrm{d}^{d} x(\tilde{\sigma}(\boldsymbol{x}))^{4}+e \cdot\left(\frac{1}{V} \int \mathrm{d}^{d} x(\tilde{\sigma}(\boldsymbol{x}))^{2}\right)^{2}
\end{aligned}
$$

with

$$
\begin{array}{ll}
a:=\frac{2}{\varrho_{0} M \chi}-\left(1-q^{2}\right), & b:=\frac{M q\left(1-q^{2}\right) \chi}{3}, \\
c:=\frac{M^{2}\left(1-q^{2}\right)\left(1-3 q^{2}\right) \chi^{2}}{24}, \quad e:=\frac{M^{2} Q\left(\left(1-q^{2}\right)^{2}-\frac{\left(1-q^{2}\right)\left(1-3 q^{2}\right)}{Q}\right) \chi^{2}}{8} .
\end{array}
$$

Due to the nonlocal biquadratic term it is, except in the symmetric case, not permitted to employ the usual Maxwell construction as it was done in [16, 17]. Instead we have to consider an ansatz for two phases with compositions $\sigma_{1,2}$ and volume fractions $v_{1,2}$ and to minimise $F$ with respect to $\tilde{\sigma}_{1,2}$ (the volume fractions can be eliminated with $v_{1}+v_{2}=0$ and with the charge conservation $v_{1} \tilde{\sigma}_{1}+v_{2} \tilde{\sigma}_{2}=$ 0 ), as was done, e.g., in ref. [22] for a similar free energy. The minimisation is performed in appendix C.5. The phase separated state is given by

$$
\sigma_{1,2}=q \varrho_{0}-\frac{b}{4 c} \pm \frac{1}{4 c} \sqrt{\frac{b^{2}(3 c+e)-8 a c^{2}}{c+e}}
$$

and

$$
v_{1,2}=\frac{1}{2} \pm \frac{b}{2 c} \sqrt{\frac{c^{2}(c+e)}{b^{2}(3 c+e)-8 a c^{2}}},
$$

with a free energy $F \propto\left(b^{2}-4 a c\right)^{2}$. The phase separation transition occurs at $a=b^{2} /(4 c)$, where $F=0$; this corresponds to $\chi=\chi_{\mathrm{h}}$ with

$$
\chi_{\mathrm{h}}=\frac{2}{M \varrho_{0}} \cdot \frac{1-3 q^{3}}{\left(1-q^{2}\right)\left(1-7 q^{2} / 3\right)} .
$$

\section{Symmetric melts}

In the symmetric case $q=0$, the phase transition point $\chi_{\mathrm{h}}$ coincides with the spinodal $\chi_{\mathrm{s}}$, corresponding to $a=0$ in eq. (2.123). For $\chi>\chi_{\mathrm{h}}$, the melt is separated in two phases of equal size and opposite composition,

$$
\sigma_{1,2}= \pm \sqrt{\frac{-2 a}{2(c+e)}}
$$

in agreement with the results of the multi-component theory to leading order in $\left(\chi-\chi_{\mathrm{s}}\right)$, see fig. $2.4(\mathrm{a})$. 


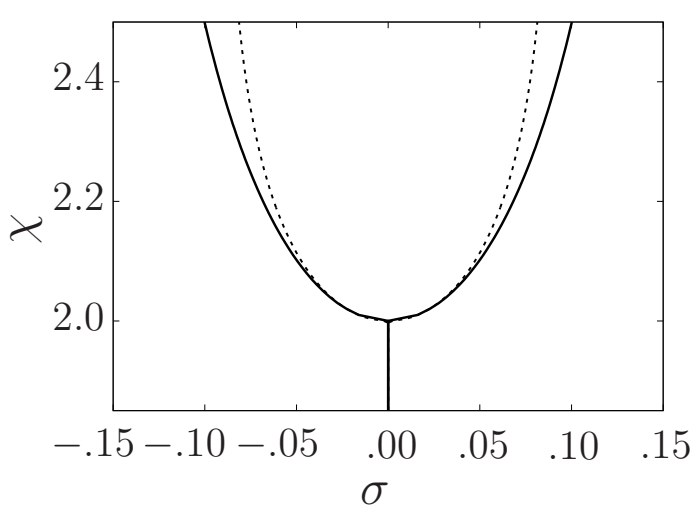

(a)

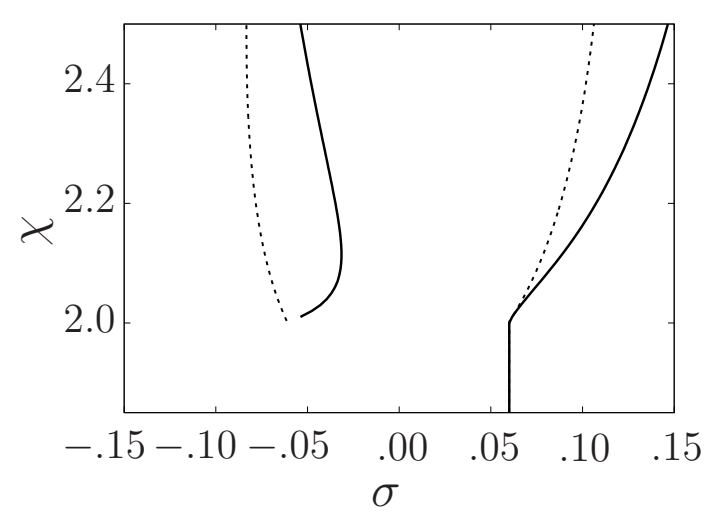

(b)

Figure 2.4.: Separation into two homogeneous phases within multi-component theory (solid lines) and Landau expansion (dotted lines). The lines indicate the mean charge density $\sigma$ of the phases. Panel (a) and (b) show the symmetric case and the case $q=0.06$, respectively.

\section{Asymmetric melts}

In the asymmetric case, with $q>0$ w.l.o.g, the phase equilibrium for $\chi=\chi_{\mathrm{h}}(1+\epsilon)$ in the vicinity of the transition is given by

$$
\begin{aligned}
& v_{1}=1-\frac{\left(1-3 q^{2}\right)\left(1-\frac{7}{3} q^{2}\right)}{4 q^{2} Q\left(\left(1-q^{2}\right)-\frac{1-3 q^{2}}{3 Q}\right)} \epsilon+\mathcal{O}\left(\epsilon^{2}\right), \\
& v_{2}=\frac{\left(1-3 q^{2}\right)\left(1-\frac{7}{3} q^{2}\right)}{4 q^{2} Q\left(\left(1-q^{2}\right)-\frac{1-3 q^{2}}{3 Q}\right)} \epsilon+\mathcal{O}\left(\epsilon^{2}\right)
\end{aligned}
$$

and

$$
\begin{aligned}
& \sigma_{1} / \varrho_{0}=q+\tilde{\sigma}_{1} / \varrho_{0}=q+\mathcal{O}(\epsilon), \\
& \sigma_{2} / \varrho_{0}=q+\tilde{\sigma}_{2} / \varrho_{0}=-\frac{1-\frac{2}{3} q^{2}-\frac{13}{3} q^{4}}{\left(1-3 q^{2}\right)^{2}} q+\mathcal{O}(\epsilon) ;
\end{aligned}
$$

the result for $q<0$ can be obtained analogously. According to Landau theory, the second phase hence comes in with zero volume and with mean compositions $\sigma_{1,2}$ being approximately symmetric about zero for not too large $q$. A comparison of both theories if shown in fig. 2.4(b) for $q=0.06$. The evolution of the new phase in the vicinity of the transition, as predicted by the Landau theory, deviates qualitatively from the Flory-Huggins results.

Nesarikar et al. [16] have argued that the discrepancy between the predictions arises from the loss of information due to the global disorder average carried out in Landau theory. We believe that this argument is incorrect: as we have shown in section 2.4.7, the descriptions of macrophase coexistence within multi-component 
and Landau theory are fully equivalent. While the multi-component theory is correct to all orders in $\sigma$, the Landau series must in practice be truncated to limit the computational effort. This restricts the range of validity to small values of the order parameters and leads to wrong predictions for first-order transitions.

\subsection{Fractionation}

In Monte-Carlo simulations [21], Houdayer and Müller observed the coexistence of a microstructured phase with two homogeneous phases over an extended range of temperatures. For the simplest example, homopolymer/diblock copolymer blends, the coexistence of homogeneous and microscopically structured phases has been studied in [4, 41]. A theory for the coexistence of micro- and macrophases in random block copolymers with uncorrelated sequences $(\lambda=0)$ and symmetric or nearly symmetric composition has been reported by Subbotin and Semenov [22]. Extending the Landau approach of ref. [17], they find that the three-phase coexistence preempts the microphase separation of the entire melt. This approach will be discussed briefly in section 2.6.8.

An important feature of the three-phase state not explicitly covered in ref. [22] is the fractionation with respect to the chain sequences. According to ref. [21], the predominantly homopolymeric chains prefer the $A$ - or $B$-rich macrophase, respectively, whereas the microstructured phase is mainly populated by copolymeric chains. The microphase separation in the third phase is faciliated or even enabled by fractionation. By expelling the homopolymeric chains, fractionation effectively increases the affinity of the residual phase to form microdomains. At the same time, the homopolymer part can form homogeneous phases with almost complete separation of $A$ and $B$. Technically, the removal of homopolymeric chains due to fractionation changes the coefficients of the Landau expansion and eventually shifts the instability of the quadratic term to finite wavelengths. This is an important difference to the established mechanism for microphase separation [17], which rests upon the subtle balance of the $k$-dependence of the quadratic and the quartic Landau coefficients, see section 2.5.5.

In the following, we shall develop a theory for the coexistence of homogeneous and microstructured phases with fractionation. Our motivation is two-fold: besides studying the three-phase coexistence, we will show that pure fractionation, i.e. the segregation of chains according to their sequences, suffices to promote microphase separation as suggested in ref. [21]. For simplicity, we concentrate on the symmetric case and use a caricature of the fractionated state, in which (only) the homopolymeric chains are allowed to separate from the rest into two macrophases that coexist with the possibly microphase separated remaining phase. By keeping the spatial dependence of the free energy only on the quadratic level, we rule out the established mechanism for microphase separation of ref. [17].

The limitation of particle exchange between the phases to strictly homopolymeric chains is severe, since their statistical weight decreases dramatically with 


\section{Random copolymer melts}

the number $Q$ of blocks per chain, and it restricts the theory to small values of $Q$. Nevertheless, the approach is able to demonstrate the potential of fractionation an alternative mechanism for microphase separation, independent of the established one discussed in section 2.5.5. Moreover, in contrast to ref. [22], our ansatz allows for the direct investigation of the partitioning of chains, since we explicitly account for different chain statistics in different phases.

\subsubsection{Landau free energy}

The basis for the investigation of macro- and microphase separation and fractionation will be the Landau expansion of the effective free energy. Like in section 2.5.4, we trace out the monomer density field and take the incompressible limit. Moreover, we let $k=0$ in the higher-order vertex functions to simplify the expressions and to rule out the conventional mechanism for microphase separation studied in section 2.5.5. The discussion will be restricted to the symmetric case $q=0$ with a replica-symmetric ansatz. The resulting Landau free energy per chain, in terms of the Fourier components $\left\{\sigma_{\boldsymbol{k}}\right\}$ of the charge density (2.59), is given by

$$
\begin{aligned}
& f\left(\left\{\sigma_{\boldsymbol{k}}\right\}\right)=\frac{1}{4 N^{2} L^{2}} \sum_{\boldsymbol{k}}^{\prime}\left(\frac{2 L^{2}}{S(k)}-\chi L\right) \sigma_{\boldsymbol{k}} \sigma_{-\boldsymbol{k}} \\
& +\frac{q_{2}^{2}-q_{4} / 3}{8 q_{2}^{4} N^{4} L^{4}} \sum_{\boldsymbol{k}_{1,2,3,4}}^{\prime} \sigma_{\boldsymbol{k}_{1}} \sigma_{\boldsymbol{k}_{2}} \sigma_{\boldsymbol{k}_{3}} \sigma_{\boldsymbol{k}_{4}} \delta_{\sum \boldsymbol{k}_{\nu}, \mathbf{0}}+\frac{q_{4}-q_{2}^{2}}{8 q_{2}^{4} N^{4} L^{4}} \sum_{\boldsymbol{k}_{1,2}}^{\prime} \sigma_{\boldsymbol{k}_{1}} \sigma_{-\boldsymbol{k}_{1}} \sigma_{\boldsymbol{k}_{2}} \sigma_{-\boldsymbol{k}_{2}}
\end{aligned}
$$

with the vertex function $S\left(k^{2}\right)$ defined in appendix C.3.2 and the average correlation of the pairs and quadruples of the charges on a chain,

$$
q_{2}:=\frac{1}{L^{2}} \sum_{s_{1,2}=1}^{L}\left[q\left(s_{1}\right) q\left(s_{2}\right)\right] \quad \text { and } \quad q_{4}:=\frac{1}{L^{4}} \sum_{s_{1,2,3,4}=1}^{L}\left[q\left(s_{1}\right) q\left(s_{2}\right) q\left(s_{3}\right) q\left(s_{4}\right)\right]
$$

respectively. The derivation of (2.135), retaining the spatial dependence of the quartic terms, is given in appendix C.6. The effective free energy (2.135) is equivalent to that in [17], except that it does not contain any approximation for long chains or small $\lambda$, and that the $k$ dependence in the quartic terms has been dropped here. The deviation from eq. (2.80) is due to a different method of tracing out the auxiliary fields; the expansions are equivalent in the vicinity of the instability of the homogeneously mixed state ( $c f$. appendix C.6).

\section{Instability}

The least stable wavenumber depends on the correlation parameter $\lambda$. Below a certain threshold $\lambda_{\mathrm{L}}$, the minimum of the quadratic coefficient is located at nonzero $k$, indicating the instability to microphase separation on increasing incompatibility. For larger $\lambda$, the minimum is located at $k=0$, corresponding to an instability with 
respect to macroscopic phase separation. The threshold $\lambda_{\mathrm{L}}$ depends on the lengths of the blocks and chains; for large $M$ and $Q$, it is given by $\lambda_{\mathrm{L}}=(\sqrt{3}-2)$ [17].

We first consider micro- and macrophase separation in the unfractionated melt; these considerations are the basis for the subsequent discussion of fractionation.

\subsubsection{Microphase separation}

As pointed out above, a melt with $\lambda<\lambda_{\mathrm{L}}$ becomes unstable with respect to microphase separation on increasing incompatibility at some point $\chi=\chi_{\mathrm{m}}$. The corresponding free energy depends on the morphology of the microphases; for simplicity, we employ a lamellar ansatz $\sigma(\boldsymbol{x}):=\sigma_{\mathrm{m}} \cos (k \boldsymbol{n} \cdot \boldsymbol{x})$ like in section 2.5.4, with an arbitrary unit vector $\boldsymbol{n}$. The amplitude $\sigma_{\mathrm{m}}$ and the wavenumber $k$ are subjected to optimisation. Inserting this ansatz into (2.135) yields

$$
f_{\mathrm{mps}}=\frac{\left(\chi_{\mathrm{m}}-\chi\right) L}{8}\left(\frac{\sigma_{\mathrm{m}}}{\varrho_{0}}\right)^{2}+\frac{q_{2}^{2}+q_{4}}{64 q_{2}^{4}}\left(\frac{\sigma_{\mathrm{m}}}{\varrho_{0}}\right)^{4} .
$$

The instability occurs at $\chi_{\mathrm{m}}=2 L / S\left(k_{\mathrm{opt}}^{2}\right)$ with an optimal wavenumber $k_{\text {opt }}$ given by the location $\operatorname{argmax}_{k>0}\left\{S\left(k^{2}\right)\right\}$ of the maximum of $S\left(k^{2}\right)$. The optimal amplitude, in principle obtained by minimisation of the effective free energy, yet bounded by physical limits, is given by

$$
\sigma_{\mathrm{m}}^{2} / \varrho_{0}^{2}=\min \left\{\frac{3 q_{2}^{3}}{q_{4}}\left(\frac{\chi}{\chi_{\mathrm{m}}}-1\right), 1\right\}
$$

for $\chi \geq \chi_{\mathrm{m}}$. The bounds $\sigma_{\mathrm{m}}= \pm \varrho_{0}$ correspond to separation into pure $A$ and $B$ at the peaks of the charge density modulation, which is the physical limit for local phase separation (see also the remark in section 2.6.4).

\subsubsection{Macroscopic phase separation}

Here and in the following we shall concentrate on the case $\lambda>\lambda_{\mathrm{L}}$, for which the copolymer melt undergoes macroscopic phase separation on increasing incompatibility at the point $\chi_{\mathrm{h}}=2 /\left(L q_{2}\right)$. Due to symmetry we may consider the separation into two phases with equal volume $V / 2$ and opposite composition $\sigma(\boldsymbol{x}):= \pm \sigma_{\mathrm{h}}$ with $\sigma_{\mathrm{h}} \geq 0$. Similar to section 2.5.7 we obtain the following effective free energy per chain for the macroscopically separated state:

$$
f_{\text {hom }}:=\frac{\left(\chi_{\mathrm{h}}-\chi\right) L}{4}\left(\frac{\sigma_{\mathrm{h}}}{\varrho_{0}}\right)^{2}+\frac{q_{4}}{12 q_{2}^{4}}\left(\frac{\sigma_{\mathrm{h}}}{\varrho_{0}}\right)^{4},
$$

The optimal charge density amplitude is determined by minimisation of $f_{\text {hom }}$ with respect to $\sigma_{\mathrm{h}}$, like in the previous section bounded by the physical limit. The limit is given by the composition $\sigma_{\max }$ of that moiety of chains that contains an above-average number of $A$ blocks. Hence,

$$
\sigma_{\mathrm{h}}^{2} / \varrho_{0}^{2}=\min \left\{\frac{3 q_{2}^{3}}{q_{4}}\left(\frac{\chi}{\chi_{\mathrm{h}}}-1\right),\left(\sigma_{\max }\right)^{2} / \varrho_{0}^{2}\right\}
$$


$\sigma_{\max }$ will be computed numerically.

\subsubsection{Free energy of fractionation}

According to the Landau expansion (2.135), microphase separation seems to be always less favourable than macroscopic demixing in the case $\lambda>\lambda_{\mathrm{L}}$ under consideration, since we have dropped the $\boldsymbol{k}$-dependence in the quartic terms which could compensate for the increase of the quadratic coefficient like in the theory of section 2.5.4. As anticipated, microphase separation can nevertheless become possible if the melt fractionates as suggested by Houdayer and Müller [20, 21], splitting into a homopolymer rich part separating macroscopically and a residual part possibly becoming able to form microphases thanks to a reduced homopolymer content.

To substantiate this mechanism, we consider a caricature of the three-phase coexistence, in which (only) the homopolymeric chains are allowed to separate from the rest. We assume that a fraction $\gamma \in[0,1]$ of the $N \cdot n_{\mathrm{hp}}=N\left(\frac{1+\lambda}{2}\right)^{Q-1}$ homopolymers in the melt is expelled from the rest to form macrophases, while the residual melt possibly undergoes microphase separation. In this case, the free energy per chain $f_{\text {tot }}^{(\mathrm{fr})}$ of the fractionated melt can be written as the weighted sum of the free energies of a macrophase separated homopolymer blend and of the remaining mixture, plus a term accounting for the entropy loss due to the partitioning:

$$
f_{\mathrm{tot}}^{(\mathrm{fr})}=\gamma n_{\mathrm{hp}} f_{\mathrm{hom}}^{(\mathrm{fr})}+\left(1-\gamma n_{\mathrm{hp}}\right) f_{\mathrm{mps}}^{(\mathrm{fr})}+f_{\mathrm{ent}}^{(\mathrm{fr})} .
$$

Fractionation will occur as soon as the excess free energy

$$
\Delta f:=f_{\text {tot }}^{(\mathrm{fr})}-f_{\text {hom }}
$$

of the fractionated melt compared to the unfractionated one becomes negative.

The entropy term $f_{\text {ent }}^{(\mathrm{fr})}$ is necessary since the influence of the system size on the free energy has been neglected so far and since the two fractions are treated as separate systems despite of the possible exchange of homopolymers. The reduction of the accessible volume, $\gamma n_{\mathrm{hp}} V$ and $\left(1-\gamma n_{\mathrm{hp}}\right) V$ instead of $V$ for the $\gamma n_{\mathrm{hp}} N$ separated homopolymer chains and the residual melt, respectively, leads to a loss of entropy. It is partly compensated for by the entropy gain due to the multitude of ways to divide the polymer chains into the two fractions $\gamma$ and $1-\gamma$. Up to constants, the net loss of entropy is given by

$$
\begin{aligned}
f_{\text {ent }}^{(\mathrm{fr})}=-\underbrace{\left(\gamma n_{\mathrm{hp}} \ln \left(\gamma n_{\mathrm{hp}}\right)+\left(1-\gamma n_{\mathrm{hp}}\right) \ln \left(1-\gamma n_{\mathrm{hp}}\right)\right)}_{\text {volume reduction }} \\
+\underbrace{n_{\mathrm{hp}}(\gamma \ln \gamma+(1-\gamma) \ln (1-\gamma))}_{\text {indistinguishability }} .
\end{aligned}
$$

The pure homopolymer fraction will separate into an $A$ - and a $B$-rich macrophase. Its free energy per chain is given by (2.139), yet with $q_{2,4}^{(\mathrm{fr})}=1, \chi_{\mathrm{h}}^{(\mathrm{fr})}=2 / L$ and 
$\sigma_{\max }^{(\mathrm{fr})}=1$, i.e. adapted to the sequence statistics of homopolymers:

$$
f_{\text {hom }}^{(\mathrm{fr})}:=\frac{\left(\chi_{\mathrm{h}}^{(\mathrm{fr})}-\chi\right) L}{4}\left(\frac{\sigma_{\mathrm{h}}^{(\mathrm{fr})}}{\varrho_{0}}\right)^{2}+\frac{1}{12}\left(\frac{\sigma_{\mathrm{h}}^{(\mathrm{fr})}}{\varrho_{0}}\right)^{4},
$$

where

$$
\left(\sigma_{\mathrm{h}}^{(\mathrm{fr})} / \varrho_{0}\right)^{2}=\min \left\{\frac{3 q_{2}^{3}}{q_{4}}\left(\frac{\chi}{\chi_{\mathrm{h}}}-1\right), 1\right\}
$$

Provided the degree of fractionation $\gamma$ is large enough, the residual fraction will separate microscopically. Its free energy per chain can be computed by analogy to section 2.6.2, yet with adapted chain statistics, i.e. with

$$
q_{2}^{(\mathrm{fr})}:=\left(1-\gamma n_{\mathrm{hp}}\right) q_{2}+\gamma n_{\mathrm{hp}}, \quad q_{4}^{(\mathrm{fr})}:=\left(1-\gamma n_{\mathrm{hp}}\right) q_{4}+\gamma n_{\mathrm{hp}}
$$

and

$$
S^{(\mathrm{fr})}\left(k^{2}\right):=\left(1-\gamma n_{\mathrm{hp}}\right) S\left(k^{2}\right)+\gamma n_{\mathrm{hp}} D\left(k^{2}\right) ;
$$

$D\left(k^{2}\right)$ denotes the discrete Debye function defined in appendix C.3.1. We obtain

$$
f_{\mathrm{mps}}^{(\mathrm{fr})}=\frac{2 L^{2} / S^{(\mathrm{fr})}\left(k_{\mathrm{opt}}^{2}\right)-\chi L}{8}\left(\frac{\sigma_{\mathrm{m}}^{(\mathrm{fr})}}{\varrho_{0}}\right)^{2}+\frac{\left(q_{2}^{(\mathrm{fr})}\right)^{2}+q_{4}^{(\mathrm{fr})}}{64\left(q_{2}^{(\mathrm{fr})}\right)^{4}}\left(\frac{\sigma_{\mathrm{m}}^{(\mathrm{fr})}}{\varrho_{0}}\right)^{4}
$$

and

$$
\left(\sigma_{\mathrm{m}}^{(\mathrm{fr})} / \varrho_{0}\right)^{2}=\min \left\{\frac{3\left(q_{2}^{(\mathrm{fr})}\right)^{3}}{q_{4}^{(\mathrm{fr})}}\left(\frac{\chi S^{(\mathrm{fr})}\left(k_{\mathrm{opt}}^{2}\right)}{2 L}-1\right), 1\right\} ;
$$

the optimal wavenumber $k_{\text {opt }}$ is given by $k_{\text {opt }}=\operatorname{argmax}_{k>0}\left\{S^{(\mathrm{fr})}\left(k^{2}\right)\right\}$. Finally, the effective free energy has to be minimised with respect to the degree of fractionation, the optimal value of which is denoted as $\gamma_{\text {opt }}$.

The choice for the maximal amplitude of the charge density in the microphases must be taken with caution: the value $\pm \varrho_{0}$ corresponds to demixing into pure $A$ and $B$ at the extrema of the sinusoidal modulation. This is only possible on length-scales smaller than the extension of the chains, since $A$ and $B$ blocks are permanently linked and can't be separated arbitrarily far. For small wavenumbers, it would thus be necessary to choose a smaller limit than one, yet it is difficult to ascertain the appropriate value. To avoid this difficulty we introduce a cutoff for $k$ larger than a minimal value $k_{\min }$, which is estimated as follows: If the chains are stretched into a linear form, however with the individual blocks retaining their unperturbed radius of gyration $R_{M}$, the maximum thickness of lamellæ having charge-density amplitude $\varrho_{0}$ is about $2 Q R_{M}$, corresponding to a minimum wavenumber of

$$
k_{\min }=\pi / Q R_{M}
$$




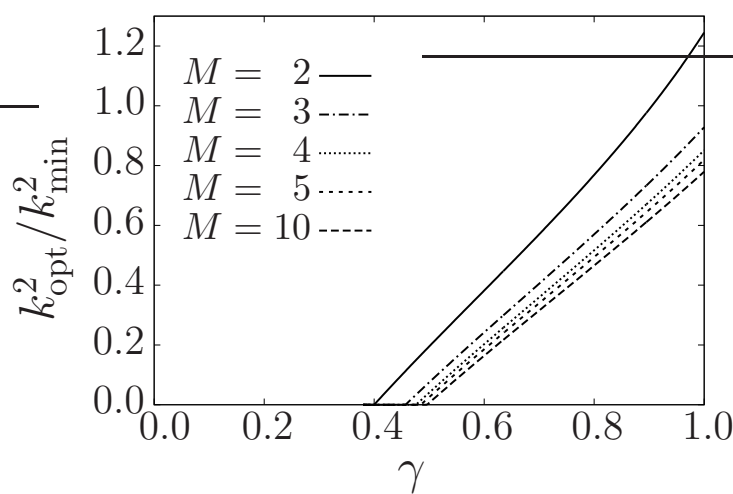

(a)

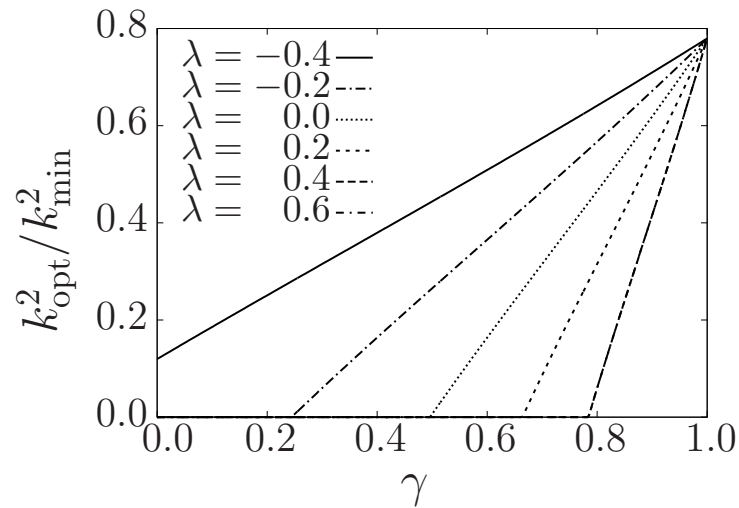

(c)

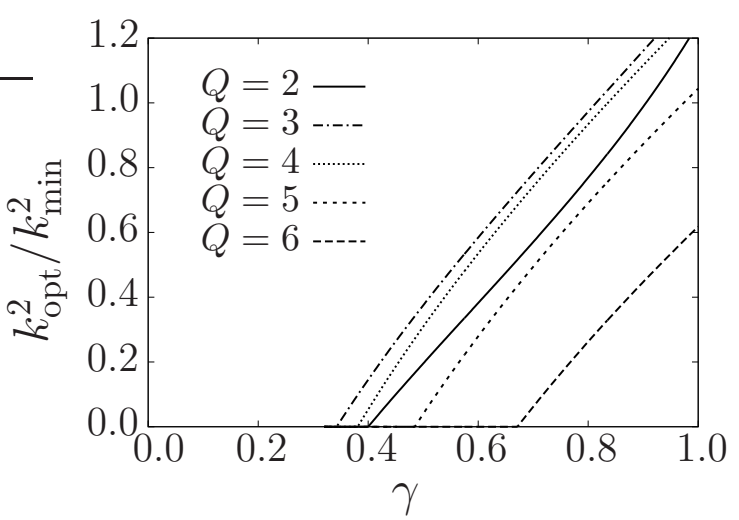

(b)

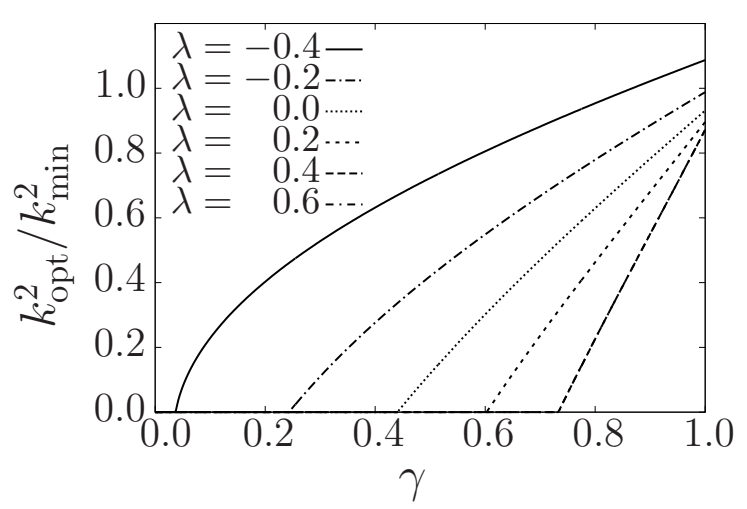

(d)

Figure 2.5.: $\quad$ Squared optimal wavenumber in units of $k_{\min }^{2}$ as a function of the degree of fractionation. (a) $Q=2, \lambda=0$, and $M=2,3,4,5,10$. (b) $M=2$, $\lambda=0$, and $Q=2,3, \ldots, 6$. (c) $Q=2, M=10$, and $\lambda=-0.4,-0.2, \ldots, 0.6$. (d) $Q=3, M=10$, and $\lambda=-0.4,-0.2, \ldots, 0.6$.

\subsubsection{Domain sizes}

Up to a certain degree of fractionation, the predicted optimal wavenumber $k_{\text {opt }}$ is zero. For small $Q$, it eventually starts to grow with increasing $\gamma$, and possibly reaches the range of sensible values $k_{\text {opt }} \gtrsim k_{\min }$. For a given number of blocks per chain, the onset of nonzero $k_{\mathrm{opt}}$ is nearly independent of the block size $M$, and $k_{\text {opt }}(\gamma)$ is roughly proportional to $k_{\min }$, i.e. to the inverse unperturbed radius of gyration of the blocks, see fig. 2.5(a). On increasing $Q$, the curve $k_{\text {opt }}(\gamma)$ is shifted towards larger $\gamma$ (apart from the step from $Q=2$ to $Q=3$ ), as illustrated in fig. 2.5(b) for $M=2, \lambda=0$ and $Q=2,3, \ldots, 7$. Beyond $Q=6$, reasonably high or even nonzero $k_{\text {opt }}$ are no longer attained at all. This is presumably due to the oversimplification of considering the fractionation of strictly homopolymeric chains only. The statistical weight of the latter decreases dramatically with increasing $Q$, so that their contribution becomes negligible. A more general model 


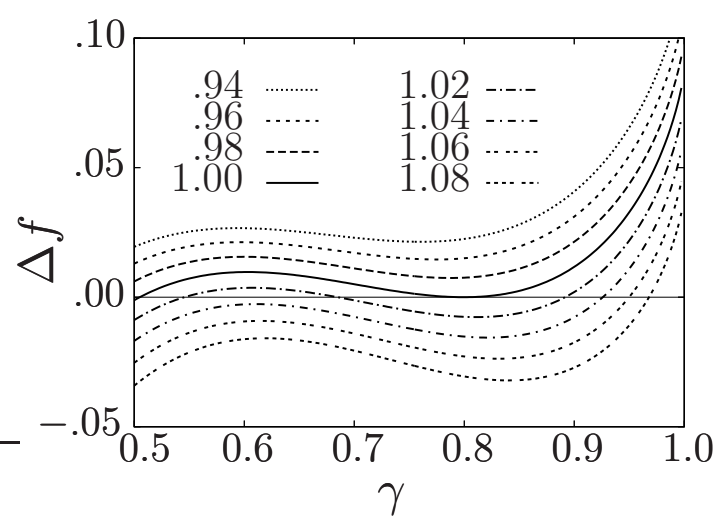

(a)

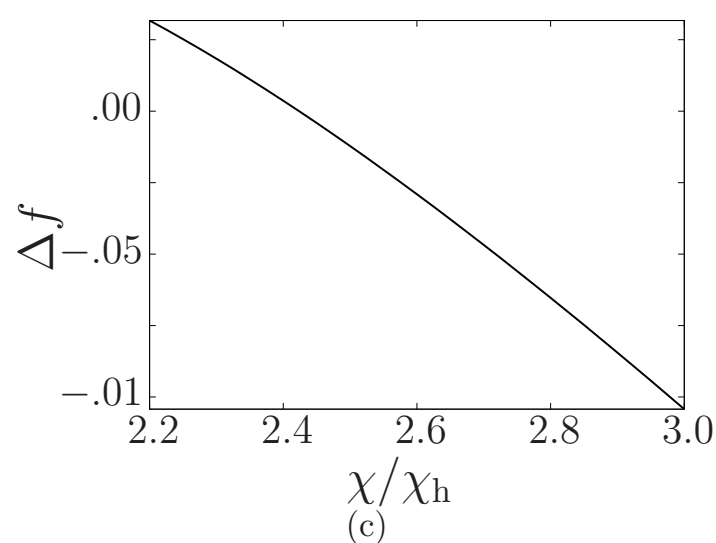

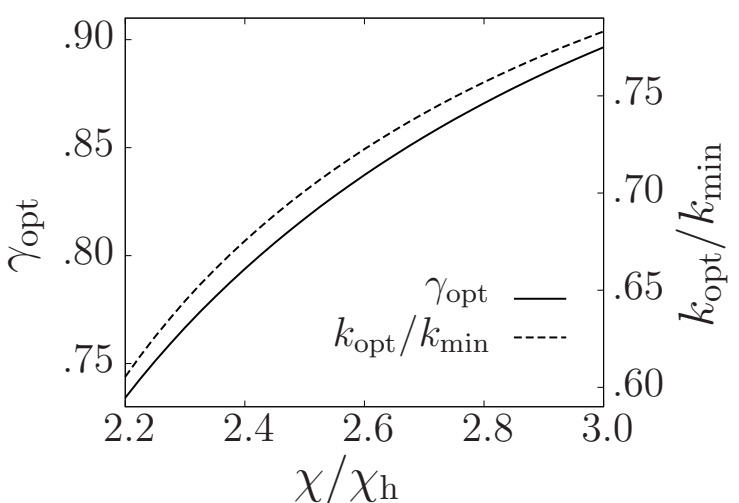

(b)

Figure 2.6.: Fractionation for $Q=2, M=10$ and $\lambda=0$. (a) Excess free energy of the fractionated state as a function of the degree of fractionation for different values of $\chi$. (b) Optimal degree of fractionation and optimal wavenumber versus $\chi / \chi_{\mathrm{h}}$. (c) Excess free energy of the fractionated state with optimal wavenumber and degree of fractionation as a function of $\chi / \chi_{\mathrm{h}}$.

should consider the fractionation of a wider class of nearly homopolymeric chains.

An increasing correlation parameter $\lambda$ reduces the fraction of alternating chains in favour of homopolymeric chains, which prefer macrophases. Consequently, the onset of nonzero wavenumbers requires a larger degree of fractionation, as shown in figs. $2.5(\mathrm{c})$ and $2.5(\mathrm{~d})$ for $Q=2$ and $Q=3$ with $M=10$.

\subsubsection{Diblocks}

For diblocks, i.e. for $Q=2$ regardless of the sequence, the effective free energy of the fractionated state reveals a local minimum at a high degree of fractionation, see fig. 2.6(a). The global minimum is located at smaller $\gamma \approx 0.4 \ldots 0.5$, at the onset of nonzero wavenumbers, where $k \ll k_{\min }$. Hence we are led to assume that the situation is more correctly described by the local minimum, which we denote 


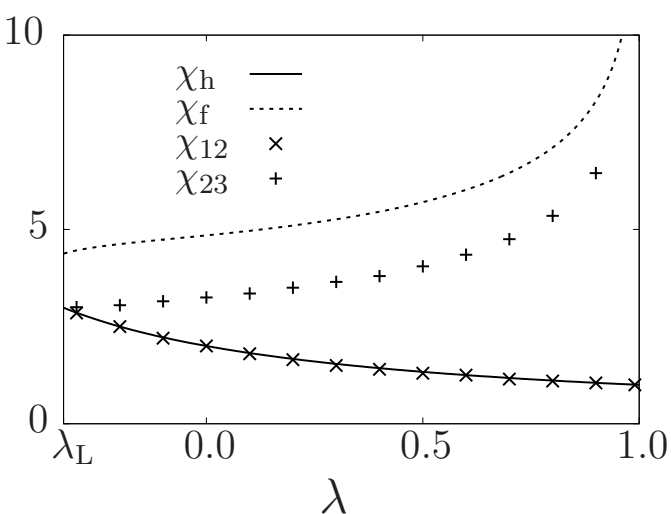

(a)

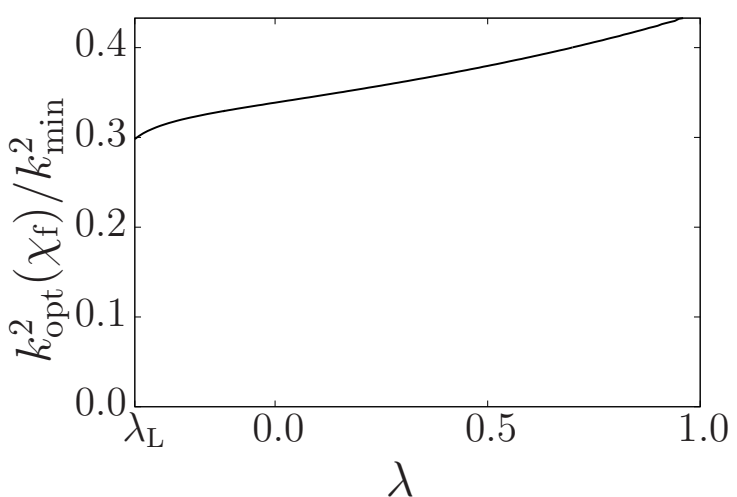

(b)

Figure 2.7.: Phase behaviour for $Q=2, M=10$ as a function of $\lambda$. (a) Onset of macrophase separation and fractionation. Lines: Landau theory; symbols: multicomponent theory (transition to two and three macrophases). (b) Squared optimal wavenumber of the microphases with fractionation.

as $\gamma_{\mathrm{opt}}$. Fig. 2.6(b) shows $\gamma_{\mathrm{opt}}$ and $k_{\mathrm{opt}}$ as a function of $\chi / \chi_{\mathrm{h}}$ for a block length of $M=10$. The corresponding excess free energy $\Delta f$ of the fractionated and microphase separated relative to the macroscopically separated state are shown in fig. 2.6(c). It suggests that fractionation sets in at about $\chi=2.4 \chi_{\mathrm{h}}$, with $\gamma \approx 0.8$ and $k \approx 0.7 k_{\min }$. Both $\gamma_{\text {opt }}$ and $k_{\text {opt }}$ grow with increasing $\chi$ : more and more of the homopolymeric chains are expelled to their separate phases, and the lamellæ in the residual phase become thinner.

With the above method we compute the onset of fractionation $\chi_{\mathrm{f}}$ as a function of the correlation parameter $\lambda$ for $\lambda>\lambda_{\mathrm{L}}=-0.33$. It is shown in fig. 2.7(a) together with the onset $\chi_{\mathrm{h}}$ of macroscopic phase separation in the unfractionated melt for $M=10$. The wavenumber characterising the microphases is shown in fig. $2.7(\mathrm{~b})$. The data for $\chi_{\mathrm{h}}$ agree well with the known results, see, for instance, ref. [4].

For sufficiently large $\lambda$, i.e. not too close to $\lambda_{\mathrm{L}}$, the data for $\chi_{\mathrm{f}}$ also show qualitative agreement with the transition from macroscopic to microstructured phases as predicted in ref. [4]. According to the latter, supported by the data of ref. [21], these microphases are, however, unfractionated in an intermediate temperature regime, with fractionation setting in at lower temperatures.

For $\lambda$ just above $\lambda_{\mathrm{L}}$, the melt is already nearly able to microphase separate, and microphase separation requires only weak fractionation. The according wavenumber is smaller than $k_{\min }$, yet this doesn't matter since the amplitude is much smaller than unity. In this range, the restriction to $k>k_{\min }$ is too rigorous; presumably this is why the computed $\chi_{\mathrm{m}}$ does not join $\chi_{\mathrm{h}}$ (and the onset $\chi_{\mathrm{m}}$ of unfractionated microphase separation) at $\lambda_{\mathrm{L}}$.

Fig. 2.7(a) includes the phase separations from one to two, and from two to three macroscopic phases as predicted by the multi-component theory of section 2.4.7. It seems that the transition to three macroscopic phases occurs earlier than frac- 


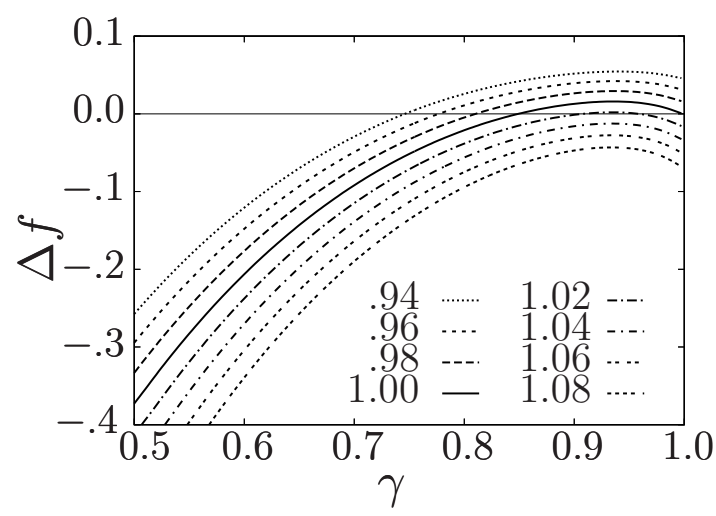

(a)

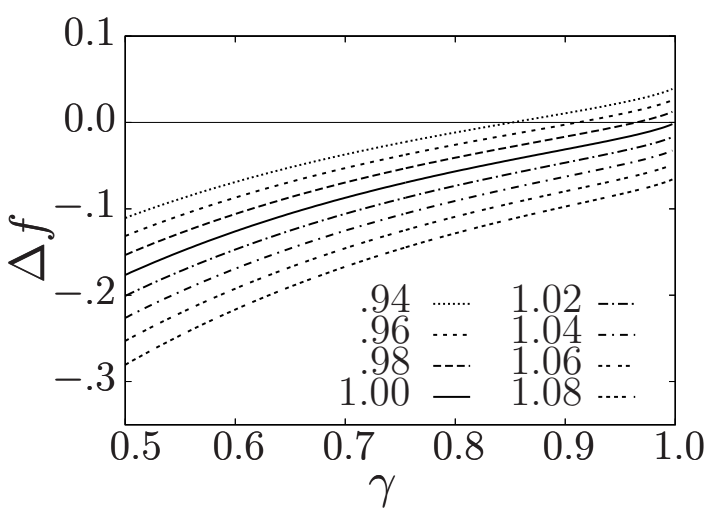

(b)

Figure 2.8.: Excess free energy of the fractionated state as a function of the degree of fractionation for different values of $\chi / \chi_{\mathrm{f}}^{*}$, with $\chi_{\mathrm{f}}^{*}$ chosen such that $\Delta f=0$ for $\chi=\chi_{\mathrm{f}}^{*}$ and $\gamma=1$. (a) $Q=3, M=10$ and $\lambda=0$. (b) $Q=4, M=10$ and $\lambda=0$.

tionation. However, our ansatz for fractionation is quite restrictive. A greater freedom would lead to a lower free energy and thus to an earlier onset of fractionation, so it is unclear whether fractionation is preceded by the coexistence of three macroscopic phases or not.

\subsubsection{Larger block numbers}

For triblocks, i.e. $Q=3$ regardless of the chain sequence, the excess free energy of the fractionated and microphase separated state with optimal wavenumber and degree of fractionation is shown in fig. 2.8(a) for $M=10$ and different values of $\chi$. The local minimum at $\gamma=1$ is not very pronounced, so it is difficult to decide whether the system is more adequately described by the local minimum at full fractionation or at some smaller value of $\gamma$ (bounded from below by the requirement $k_{\mathrm{opt}} \gtrsim k_{\mathrm{min}}$ ). For larger numbers of blocks, i.e. for $Q$ between 4 and 7, the excess free energy no longer exhibits a local minimum at large $\gamma$ at all, see fig. 2.8(b) for $Q=4$ (the graphs for $Q=5$ and $Q=6$ are shifted to higher $\gamma$, but otherwise look similar). To avoid this difficulty, which is presumably due to the too restrictive ansatz for fractionation, we abstain from optimising with respect to $\gamma$ for $Q \geq 3$ in the following. Instead, we keep $\gamma$ fixed and minimise the free energy just with respect to the amplitudes of the charge density and the wavenumber $q$ describing the microphases.

The resulting onset of fractionation is plotted in fig. 2.9. Panel (a) shows $\chi_{\mathrm{f}}$ as a function of $\lambda$ for $Q=3, M=10$ and different choices of $\gamma=0.8,0.9,0.95,0.99$ and 1 . The curves have been cut off for $k_{\mathrm{opt}}<0.7 k_{\text {min }}$. Apparently, the results for $\gamma=1$ yield a good estimate for $\chi_{\mathrm{f}}$ over the whole range of $\lambda>\lambda_{\mathrm{L}}=-0.45$. Panel (b) shows $\chi_{\mathrm{f}}$ versus $\gamma$ for $Q=4$ and $M=2,3,5$ and 10. Like fig. 2.8(b) it suggests that the optimal $\gamma$ is rather small, yet $\gamma$ is bounded from below by the 


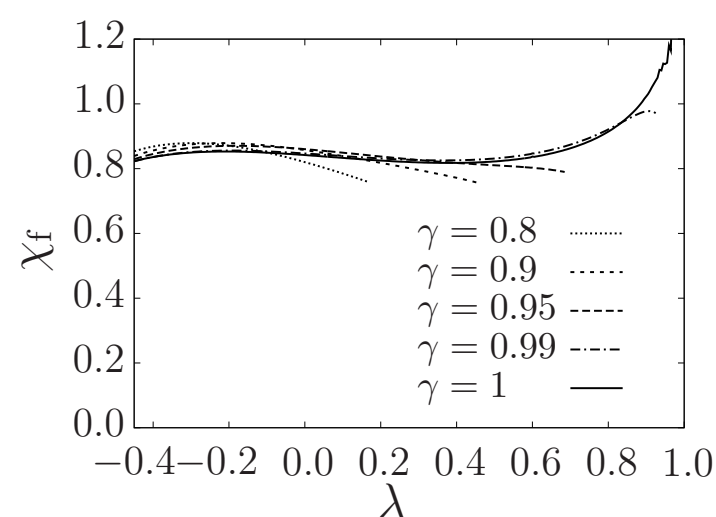

(a)

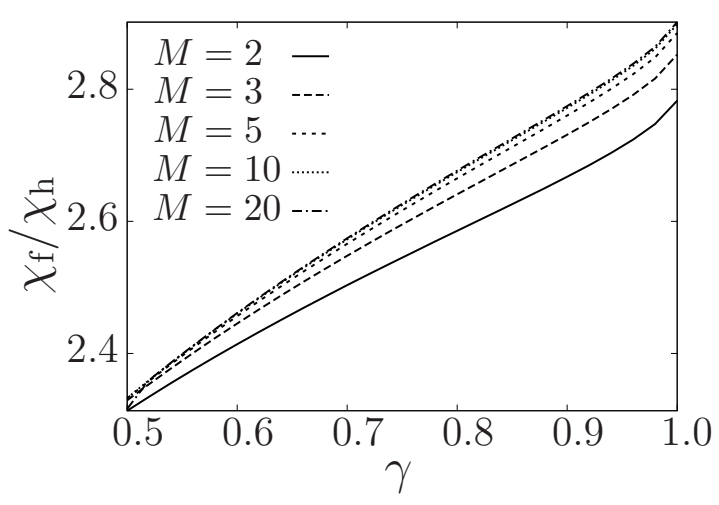

(b)

Figure 2.9.: Onset of fractionation for fixed degree of fractionation. (a) $\chi_{\mathrm{f}}(\lambda)$ for $Q=3, M=10$ and $\gamma=0.8,0.9,0.95,0.99$ and 1 , cut off at $q / k_{\min }<0.7$. (b) $\chi_{\mathrm{f}}(\gamma)$ for $Q=4$ and $M=2,3,5$ and 10 .

requirement of a sufficiently large wavenumber $k_{\mathrm{opt}} \gtrsim k_{\min }, c f$. section 2.6.4.

The coexistence of a microstructured phase with two macrophases found here was also observed in [21] for $Q=3$. For technical reasons, it was only studied for the cases $Q=2$ and 3 therein.

\subsubsection{Implicit fractionation}

In the following we outline an alternative approach to three-phase coexistence, suggested and discussed for uncorrelated sequences $(\lambda=0)$ by Subbotin and Semenov [22]. For simplicity, we confine ourselves to the symmetric case $q=0$. The starting point is the effective free energy density

$$
\begin{aligned}
f\left(\left\{\sigma_{\boldsymbol{k}}\right\}\right) / Q=\frac{2}{(2 N L)^{2}} \sum_{\boldsymbol{k}}^{\prime} & \left(\frac{M k^{2}}{3}-\frac{\chi M}{2}+1\right) \sigma_{\boldsymbol{k}} \sigma_{-\boldsymbol{k}} \\
+ & \frac{4}{3(2 N L)^{4}} \sum_{\boldsymbol{k}_{1,2,3,4}}^{\prime} \sigma_{\boldsymbol{k}_{1}} \sigma_{\boldsymbol{k}_{2}} \sigma_{\boldsymbol{k}_{3}} \sigma_{\boldsymbol{k}_{4}} \delta_{\sum \boldsymbol{k}_{\nu}, \mathbf{0}} \\
& +\frac{4(Q-1)}{(2 N L)^{4}} \sum_{\boldsymbol{k}_{1,2}}^{\prime} g_{\mathrm{D}}\left(L\left(k_{1}^{2}+k_{2}^{2}\right)\right) \sigma_{\boldsymbol{k}_{1}} \sigma_{-\boldsymbol{k}_{1}} \sigma_{\boldsymbol{k}_{2}} \sigma_{-\boldsymbol{k}_{2}}
\end{aligned}
$$

which differs from (2.135) by the expansion of the second-order vertex functions for small arguments and long blocks and chains, and by the restored $k$-dependence of the biquadratic term. Subbotin and Semenov consider the ansatz

$$
\sigma(\boldsymbol{x})= \begin{cases}+\sigma_{0} & \text { for } \boldsymbol{x} \in \mathbb{V}_{1}, \\ -\sigma_{0} & \text { for } \boldsymbol{x} \in \mathbb{V}_{2}, \\ \sigma_{\mathrm{m}} \cdot \cos (\boldsymbol{k} \boldsymbol{x}) & \text { else }\end{cases}
$$


in real space, with two macroscopic phases in the subvolumes $\mathbb{V}_{1}$ and $\mathbb{V}_{2}$ of equal size (for reasons of symmetry) and a lamellar microphase in the remaining volume. The volume fraction $p_{\mathrm{m}}$ of the microphase is considered to be small. After insertion of the ansatz (2.152), the effective free energy (2.151) is expanded to first order in $p_{\mathrm{m}}$ and minimised with respect to $p_{\mathrm{m}}, \sigma_{0}$ and $\sigma_{\mathrm{m}}$ in this approximation.

According to the above approach of ref. [22], the copolymer melt first separates into two macroscopic phases at $\chi_{\mathrm{h}}=2 / M$. The lamellar third phase starts to grow at $\chi_{\mathrm{f}}=\chi_{\mathrm{h}}\left(1+Q^{-1}+0.474 Q^{-3 / 2}\right)$ with initially zero volume and nonzero amplitude of the order $1 / Q$. More complicated microphases are predicted to appear on further cooling.

\subsubsection{Summary and discussion}

In this section, we have studied the coexistence of macro- and microphases with fractionation, i.e. the partitioning of the chains according to their sequences, using a simple picture of the fractionated state, in which (only) the homopolymeric chains were allowed to separate from the rest. Because of this simplification, the model is restricted to chain lengths of up to five blocks per chain, since the statistical weight of the strictly homopolymeric chains decreases rapidly with $Q$. Furthermore, we were forced to introduce a large-scale cutoff for the domain sizes to prevent the theory from predicting unphysically large degrees of separation. Due to the approximations, the three-phase coexistence could not be computed with as good accuracy as in ref. [22].

We have demonstrated that fractionation constitutes an alternative mechanism of enabling microphase separation. In the established theory by Fredrickson et al. [17], the instability of the quadratic term of the Landau expansion is always located at $k=0$, and microphase separation is only possible due to the subtle balance of the $k$-dependences of the quadratic and the quartic term. To rule out this possibility, we have dropped the $k$-dependence of the Landau coefficients except on the quadratic level. Fractionation allows the chains to sort themselves according to their preference of micro- or macrophases. From the point of view of the potential microphase, the homopolymers are sorted out, which increases the affinity to microphase separation, eventually shifting the instability of the Landau expansion to finite wavelengths. The homopolymer rich part, on the other hand, can separate macroscopically, yielding almost complete separation of $A$ and $B$. As we have shown, the free energy gain due to macro- and microphase separation outweighs the loss of entropy due to fractionation at sufficiently low temperature. Moreover, our approach, in contrast to that of ref. [22], allows for the direct observation of fractionation, since the different chain statistics in different phases are considered explicitly. 


\subsection{Discussion and conclusions}

Starting from the microscopic description of random block copolymer melts suggested by Fredrickson et al. [17], we have derived an effective free energy for this system in two different formulations, averaged over the quenched randomness by means of the replica method: First, in terms of component densities, classifying all chains with respect to their overall composition, disregarding their sequence and their conformation and thus yielding a coarse-grained description. Second, in terms of the local density of monomers and the charge density, i.e. the local difference in the concentrations of $A$ and $B$ monomers.

For incompressible melts, we have shown the multi-component free energy to be equivalent to the free energy of ref. [15] usually obtained with a Flory-Huggins approach, and we have recovered the results of Nesarikar et al. [16] for the coexistence of multiple homogeneous phases. The phase equilibria have been computed using the algorithm proposed in [15]. Our derivation establishes the link to the alternative formulation in terms of the particle and charge density obtained from the same model. Like in [17], this non-coarse-grained formulation was the basis for a Landau expansion approach capable of describing both fundamental mechanisms of phase separation in a random copolymer system: the separation into macroscopic phases and the formation of a microscopically structured phase. Although focusing on the incompressible case, our derivation initially retains effects of compressibility. For the incompressible case we obtained a free energy similar, but not identical, to that of ref. [17]. Substantial differences arise from certain approximations made in [17]: aiming at the case of a high number $Q$ of blocks per chain and a large block size $M$, Fredrickson et al. neglected terms in the free energy appearing subdominant in $1 / Q$. The potential gain in the free energy associated with microphase separation is, however, of the order $1 / Q$, as well, hence the neglect of these terms is unjustified. With an analytical approximation based on the full free energy expansion, we found that microphases set in with finite wavelength, in contrast to the prediction in [17]. The analytic approximation has been complemented with data obtained by numerical minimisation of the free energy expansion. For very small "blocks" of just a single monomer, i.e. $M=1$, the theory predicts the phase behaviour to be highly sensitive to the sequence correlation: the melt either remains homogenous or microphase separates on the smallest possible length-scale, which is bounded from below by the diameter of a monomer.

In the limit of infinite block and chain lengths, we recover the results of Fredrickson et al. [17], apart from the microphase wavelength at the transition. In the case of symmetric chains and uncorrelated sequences, the spinodal temperature is proportional to the block length, and the temperature range of two-phase coexistence is of the order $1 / Q$. The microphases set in with large domain spacing and become narrower on going deeper into the microphase separated regime. In polyurethanes with suppressed crystallisation, Ryan et al. [31] measured the domain spacing as a function of temperature for two different chain lengths by means of small-angle X-ray scattering. Due to a considerable polydispersity of block and 
chain lengths, the microphase transition was stretched over a finite temperature interval. Ryan et al. observed that the domain spacing, after an increase during the transition attributed to chain stretching, decreases with decreasing temperature in agreement with the theory. Moreover, the transition temperature was found to increase with the molecular weight. However, the prepolymer blocks used in ref. [31] reveal a considerable amount of polydispersity, and the two components have molecular weights differing by nearly a factor of 20; thus it is difficult to compare the results directly to the ideal case of the theory discussed here.

Coarse-graining the theory in terms of monomer and charge densities, which allows for using the exact free energy instead of an expansion, we have shown the equivalence to the multi-component theory of ref. [16] for multiphase equilibria. The multi-component theory is correct to all orders in the local composition, hence leads to the proper mean-field description of the coexistence of two (or more) phases. By comparison we found, in accordance with ref. [16], that the Landau theory based on the fourth-order expansion accurately describes the two-phase coexistence to leading order for the symmetric case, yet fails for asymmetric melts, where the transition is of first order. In the latter case, the range of validity of the Landau expansion is exceeded because of the finite jump of the order parameter.

Monte-Carlo simulations by Houdayer and Müller suggest the possibility of the coexistence of homogeneous and microstructured phases, dependent on the fractionation of the chains according to their sequence: homopolymeric chains prefer macrophases (rich in $A$ or $B$ ), copolymeric chains prefer the microstructured phase [21]. A Landau theory of the three-phase state, however without explicit consideration of fractionation, was given by Subbotin and Semenov [22]. To study said three-phase coexistence, especially the effects of fractionation, we have developed and employed a caricature of the three-phase state, in which the homopolymeric chains were allowed to "choose" between micro- and macrophases. Because of the rapid decrease in the statistical weight of the strictly homopolymeric chains with the number $Q$ of blocks per chain, this simplified picture is limited to small values of $Q$. We have shown that fractionation is sufficient to enable microphase separation, since the homopolymeric chains hampering microphase separation are sorted out into the macrophases, thus increasing the affinity to microdomain formation in the remainder. In particular, we have shown that this mechanism is an alternative to the theory of Fredrickson et al. [17], in which a subtle balance of the spatial dependence of the quadratic and fourth-order terms in the Landau expansion is required for microphase separation. 
2. Random copolymer melts 


\section{Crosslinked homopolymer blends}

\subsection{Introduction}

Blends of chemically distinct polymers are usually incompatible [42], i.e. they have a strong tendency to phase separate, since the entropy of mixing, compared to the mixture of monomers, is suppressed by a factor of the degree of polymerisation, so that small repulsive forces are effectively amplified. These systems can be addressed, for instance, with the Flory-Huggins theory [14, 37-40], originally developed for homopolymers in solution, but applicable to molten homopolymer blends, as well [43]. For symmetric blends consisting of two species in equal amounts, mean-field theory predicts a second-order transition to macroscopic phases at a critical temperature $T_{0}$. The transition, however, is rendered first order by fluctuations [44]; for asymmetric blends it is first order, anyway.

As first pointed out by de Gennes [45], crosslinks introduced at high temperatures, in the homogeneously mixed state, counteract and possibly inhibit macroscopic phase separation. With a Landau free energy derived by an analogy to polarisation in a dielectric medium, de Gennes showed that strong crosslinking in the well mixed blend enhances the stability of the homogeneous phase. Macroscopic demixing is replaced with microphase separation occurring at a critical incompatibility, corresponding to a demixing temperature $T_{\mathrm{c}}<T_{0}$ that decreases linearly with the number of crosslinks. According to this theory, the scattering intensity in the mixed state reveals a maximum at a nonzero wavenumber of the order of the inverse mesh size of the network and vanishes for $q \rightarrow 0$ and $q \rightarrow \infty$. As the microphase separation temperature is approached, the peak diverges as $1 /\left(T-T_{\mathrm{c}}\right)$, and its location anticipates the length-scale of the microdomains. The issue of mixing and the size and morphologies of microdomains is of practical importance in the design of polymer blends, which can exhibit mechanical and optical properties superior to those of the pure component, since these properties also depend on the microscopical structure; see, e.g., [46-48].

Experimental investigations were performed by Briber and Bauer on blends of deuterated polystyrene and poly-vinylmethylether (dPS/PVME) in a near-critical composition. The samples were crosslinked in the mixed state by $\gamma$-ray irradiation to different degrees and measured by small angle neutron scattering (SANS) [49]. The results largely confirmed de Gennes' predictions, with deviations in the proportionality constants probably due to the very approximate derivation of de Gennes' 


\section{Crosslinked homopolymer blends}

theory - apart from the small wavenumber behaviour of the scattering function. In contrast to the prediction, the latter does not vanish at $q=0$. Briber and Bauer conjectured that the residual zero-angle intensity is due to concentration fluctuations present at the time of crosslinking, "frozen-in" in the network. Similar experiments were performed by Jinnai et al. [50] on blends of deuterated and protonated polybutadiene $(\mathrm{dPB} / \mathrm{hPB})$, crosslinked by thermally decomposed peroxide. Unlike Briber and Bauer, Jinnai et al. found only a very small temperature dependence in the SANS profiles of the crosslinked blends, which they attributed to having measured far away from the microphase separation temperature that was out of reach due to strong crosslinking and due to the weak temperature dependence of the incompatibility of $\mathrm{dPB} / \mathrm{hPB}$.

de Gennes' model has been modified by various authors regarding different aspects, in particular the issue of the small $q$ scattering intensity. To account for the fluctuations present prior to preparation and preserved in the gel, Benhamou et al. [51] refined the analogy to a dielectric by including a Debye-Hückel screening of the "charges", which permits long-range inhomogeneities and leads to a non-vanishing zero-angle scattering. The screening length is determined selfconsistently, by assuming that the scattering intensity at $q=0$ is not affected by the crosslinking as long as the temperature remains unchanged after preparation [52]. Approaching microphase separation, the computed scattering intensity develops a peak that finally diverges as predicted in [45]. The $q=0$ limit is nonzero by construction, and far enough above $T_{\mathrm{c}}$, the function decreases monotonically.

In an alternative approach, Read et al. considered a blend of polymer chains anchored at both ends to randomly chosen fixed points in space [53] in order to account for the pinning of chains due to the crosslinks. With an RPA calculation, they obtain a scattering function revealing the desired nonzero value at $q=0$, which is also shown to originate partly from frozen fluctuations. In contrast to de Gennes' prediction, the peak evolving close to microphase separation diverges as $1 /\left(T-T_{\mathrm{c}}\right)^{2}$ instead of $1 /\left(T-T_{\mathrm{c}}\right)$. Read et al. furthermore investigate the effects of asymmetric compositions and of applied strain. Studies of crosslinked systems, based on the microscopic model by Panyukov and Rabin [54], were reported by Sfatos and Shakhnovich [55]; as far as homopolymer blends are concerned, these authors, however, do not go beyond de Gennes' results.

Monte Carlo studies of crosslinked polymer blends on a lattice were performed by Lay et al. [56]. During crosslinking, the incompatibility interaction was turned off, corresponding to a well mixed blend far from phase separation. The authors find qualitative agreement with de Gennes' predictions at finite wavenumber, with a peak in the scattering function. The corresponding length-scale is, however, twice as large as expected, which Lay et al. attribute to larger cooperative rearrangements on scales beyond the strand length. They also find slight contractions of strands connecting chains of the same type " $A A A$ " and extensions of strands connecting chains of the opposite type " $A B A$ ".

The critical behaviour of the original model by de Gennes (not accounting for pre-crosslinking fluctuations) was examined in [57] in a renormalisation group ap- 
proach. Stress-induced ordering and effects of strain have been studied in [53], [58] and [59]. Stepanow and coworkers extended the model in [45] by an inhomogeneous rigidity (treated as annealed) to account for the disorder of the crosslinks [60]. The importance of the latter was also stressed in [58] and [61], pointing out that it destroys a potential long-range order in the microphases. The kinetics of microphase separation has been assessed in [62]. An early review of crosslinked polymer blends can be found in [63]. Related systems are, e.g., crosslinked blends in solution [64], non-permanently crosslinked polymer blends [65], interpenetrating and semi-interpenetrating networks, [66-69] and [70, 71], crosslinked diblock copolymer melts [72, 73], nematic elastomers [74] and mixtures of crosslinked polymers with nematic liquid crystals [75].

Our starting point is a Landau expansion for the free energy in terms of two order parameters: the local imbalance of the concentrations of the two polymer species associated with phase separation, and the local static density correlations signalling amorphous solidification. The free energy is derived from a model extending previous work on the gelation of homopolymers [76, 77]. The model covers both demixing and gelation in a microscopic fashion, allowing to treat the freezingin of fluctuations and microphase separation without ad hoc assumptions. As it turns out, the crosslinked blend is controlled by three parameters, the chemical potential of the crosslinks and the incompatibilities (or inverse temperatures) at the instants of crosslinking and measurement. These parameters set three lengthscales, which rule the phase diagram and the scattering functions, viz. the mean localisation length in the gel and the correlation lengths of critical demixing fluctuations prior to crosslinking and at the time of measurement. We calculate the degree of stabilisation of the mixed regime due to crosslinking and discuss the neutron and X-ray scattering functions in the mixed state, including the region close to microphase separation. To achieve a rough understanding of the microphase separated state itself close to the transition, we determine the amplitude and the wavelength, assuming simple lattices structures for the separation pattern as a caricature of the real microphase state: lamellæ, hexagonally ordered cylinders and spheres on a bcc lattice. Since the randomly crosslinked system is unlikely to reveal long-range order [58, 61], we also consider a random morphology with a characteristic wavelength. The optimal morphology is determined taking into account the compositional asymmetry and the compressibility of the blend. Our calculations are performed on the level of mean-field and Gaussian approximation. As pointed out by Benhamou et al. [64], the nonclassical region is small, hence the mean-field approximation should be largely valid. A short account of our main results is given in [78]; the microscopic derivation and a discussion of the effects of asymmetry and compressibility are reported in [79]. 


\subsection{Model}

In the following, we shall extend the model for homopolymers laid out in section 2.2.1 to include the incompatibility of the two homopolymer species blended together. In the style of [77], we shall also account for the constraints introduced by crosslinking, which, together with the entropy of mixing, counteract the phase separation driven by the incompatibility. The crosslinks obviously generate branched structures; yet, the polymers in the initial polymer blend are still assumed to be linear chains.

\subsubsection{Homopolymer species and incompatibility}

Even aside from their distinct "identities", the monomers of the two species in the blend will generally not have the same properties. However, our aim is to study the interplay of demixing and gelation, and not the effect of different degrees of polymerisation or occupied volumes, for example. Hence it is permitted to simplify the picture by assuming that the species merely differ in their specific interaction. The model from section 2.2 .1 can be retained nearly without modification, apart from the inclusion of incompatibility.

Instead of indicating the monomer types by assigning charges, as it is necessary in the case of random copolymers due to the huge number of possible sequences, the two chain types can just be treated separately. We consider a melt containing $N_{A}$ and $N_{B}$ chains of type $A$ and $B$. The monomer positions are given by $\boldsymbol{R}_{a, i}(s)$, where $a=A, B$ denotes the species, $i=1, \ldots, N_{a}$ the chain number and $s=1, \ldots, L$ the location within the chain. In analogy to the copolymers of section 2.2 .2 , the incompatibility of the two species is modelled by the Hamiltonian

$$
H^{\mathrm{C}}:=-\frac{\chi}{2} \sum_{a_{1,2}=A, B} \sum_{i_{1}=1}^{N_{a_{1}}} \sum_{i_{2}=1}^{N_{a_{2}}} \sum_{s_{1,2}=1}^{L}\left(2 \delta_{a_{1}, a_{2}}-1\right) W\left(\left|\boldsymbol{R}_{a_{1}, i_{1}}\left(s_{1}\right)-\boldsymbol{R}_{a_{1}, i_{2}}\left(s_{2}\right)\right|\right) .
$$

Each monomer pair in the melt yields an attractive or repulsive contribution depending on whether the monomers are of the same type or not. It is weighted according to the distance by a local function $W(R)$, which is taken to be normalised by $\int \mathrm{d} V W(|\boldsymbol{R}|)=1$. The incompatibility parameter $\chi$ sets the magnitude of the interaction, being one of the principal control parameters in the system.

\subsubsection{Brownian chain model}

Since homopolymer chains are essentially featureless and homogeneous along the contour - there are no charge patterns as in copolymers, we may switch to the Brownian chain model with continuous instead of discrete chains. This corresponds to taking the continuum limit of an infinite number of infinitely small monomers per chain, while retaining the original radius of gyration of the polymer. The discrete monomer index $s$ is substituted by a continuous arclength 
variable $t:=s / L \in[0,1]$, and the sums and difference quotients are replaced by integrals and derivatives,

$$
\sum_{s=1}^{L} f(s) \rightarrow L \cdot \int_{0}^{1} \mathrm{~d} t f(L t)
$$

and

$$
\boldsymbol{R}_{a, i}(s+1)-\boldsymbol{R}_{a, i}(s) \rightarrow \frac{1}{L} \frac{\mathrm{d} \tilde{\boldsymbol{R}}_{a, i}(t)}{\mathrm{d} t} \text { with } \quad \tilde{\boldsymbol{R}}_{a, i}(t):=\boldsymbol{R}_{a, i}(L t) .
$$

Adapted to the blend of continuous chains, the Hamiltonians (2.2), (2.3) and (3.1) for chain connectivity, excluded volume and incompatibility read

$$
\begin{aligned}
H^{\mathrm{W}} & =k_{\mathrm{B}} T \cdot \frac{d}{2 L b^{2}} \sum_{a=A, B} \sum_{i=1}^{N_{a}} \int_{0}^{1} \mathrm{~d} s\left(\frac{\mathrm{d} \tilde{\boldsymbol{R}}_{a, i}(s)}{\mathrm{d} s}\right)^{2}, \\
H^{\mathrm{EV}} & =k_{\mathrm{B}} T \cdot \frac{\lambda L^{2}}{2} \sum_{a_{1,2}=A, B} \sum_{i_{1}=1}^{N_{a_{1}}} \sum_{i_{2}=1}^{N_{a_{2}}} \int_{0}^{1} \mathrm{~d} s_{1} \mathrm{~d} s_{2} U\left(\left|\tilde{\boldsymbol{R}}_{a_{1}, i_{1}}\left(s_{1}\right)-\tilde{\boldsymbol{R}}_{a_{1}, i_{2}}\left(s_{2}\right)\right|\right)
\end{aligned}
$$

and

$$
\begin{aligned}
H^{\mathrm{C}}=-\frac{\chi L^{2}}{2} \sum_{a_{1,2}=A, B} \sum_{i_{1}=1}^{N_{a_{1}}} \sum_{i_{2}=1}^{N_{a_{2}}} \int_{0}^{1} \mathrm{~d} s_{1} \mathrm{~d} s_{2} & \left(2 \delta_{a_{1}, a_{2}}-1\right) \\
& \times W\left(\left|\tilde{\boldsymbol{R}}_{a_{1}, i_{1}}\left(s_{1}\right)-\tilde{\boldsymbol{R}}_{a_{1}, i_{2}}\left(s_{2}\right)\right|\right),
\end{aligned}
$$

where the arclength variable $t$ has been renamed $s$. Deviating from the notation of chapter 2, the excluded volume parameter is denoted as $\lambda$ instead of $\mu$ here to avoid the collision with the parameter $\mu$ related to crosslinking, which will be introduced in section 3.2.4.

\subsubsection{Dimensionless representation, choosing the energy scale}

To simplify the expressions it is convenient to introduce dimensionless positional variables

$$
\boldsymbol{r}_{a, i}(s)=\sqrt{\frac{2 d}{L b^{2}}} \tilde{\boldsymbol{R}}_{a, i}(s),
$$

so that all lengths are measured in units of the radius of gyration, up to a numerical factor of order one depending on the dimensionality of space. The rescaled sample volume is $V^{\prime}=\left(2 d / L b^{2}\right)^{d / 2} V$. The normalisation of $U$ and $W$ is rescaled similarly, yet can be restored by introducing $U^{\prime}(r):=\left(L b^{2} / 2 d\right)^{d / 2} U(R)$ 
and $W^{\prime}(r):=\left(L b^{2} / 2 d\right)^{d / 2} W(R)$, which generates a pre-factor of $V^{\prime} / V$ in the excluded volume and incompatibility interaction. In anticipation of the results of the subsequent analysis, to make the basic quantities look as simple as possible later, we substitute

$$
\lambda=: \frac{V}{V^{\prime}} \cdot L^{2} \varrho_{0}^{\prime} \cdot \lambda^{\prime} \quad \text { and } \quad \chi=: \frac{V}{V^{\prime}} \cdot L^{2} \varrho_{0}^{\prime} \cdot k_{\mathrm{B}} T \cdot \chi^{\prime},
$$

where

$$
\varrho_{0}^{\prime}:=\frac{\left(N_{A}+N_{B}\right)}{V^{\prime}}=\frac{N}{V^{\prime}}
$$

denotes the number density of chains with respect to the rescaled volume, and with $N:=N_{A}+N_{B}$ the total number of chains.

Like in the case of random copolymers, we choose the new parameter $\chi^{\prime}$ such that the incompatibility Hamiltonian is formally proportional to $k_{\mathrm{B}} T$ like $H^{\mathrm{W}}$ and $H^{\mathrm{EV}}$. In the Boltzmann weights $\exp (-\beta H)$, with $H$ being the sum of all three Hamiltonians, $\beta$ and $k_{\mathrm{B}} T$ cancel. The explicit temperature dependence of the partition function is eliminated, so that temperature as an independent parameter can be dropped. Since the temperature nevertheless influences the system via $\chi^{\prime}(T) \propto 1 / T$, the parameter $\chi^{\prime}$ will also be referred to as the inverse temperature. Effectively, we have chosen the energy scale according to $k_{\mathrm{B}} T=1$, without giving up the ability to control the temperature.

To keep the notation simple, we drop the primes indicating the rescalings of $V, \rho, \chi$ and $\lambda$. Expressed in the new variables, the Wiener Hamilton and the Hamiltonians of excluded volume and incompatibility then read

$$
\begin{aligned}
H^{\mathrm{W}} & =\frac{k_{\mathrm{B}} T}{4} \sum_{a=A, B} \sum_{i=1}^{N_{a}} \int_{0}^{1} \mathrm{~d} s\left(\frac{\mathrm{d} \boldsymbol{r}_{a, i}(s)}{\mathrm{d} s}\right)^{2}, \\
H^{\mathrm{EV}} & =k_{\mathrm{B}} T \cdot \frac{\lambda}{2 \varrho_{0}^{\prime}} \sum_{a_{1,2}=A, B} \sum_{i_{1}=1}^{N_{a_{1}}} \sum_{i_{2}=1}^{N_{a_{2}}} \int_{0}^{1} \mathrm{~d} s_{1} \mathrm{~d} s_{2} U^{\prime}\left(\left|\boldsymbol{r}_{a_{1}, i_{1}}\left(s_{1}\right)-\boldsymbol{r}_{a_{1}, i_{2}}\left(s_{2}\right)\right|\right)
\end{aligned}
$$

and

$$
\begin{aligned}
H^{\mathrm{C}}=-k_{\mathrm{B}} T \cdot \frac{\chi}{2 \varrho_{0}^{\prime}} \sum_{a_{1,2}=A, B} \sum_{i_{1}=1}^{N_{a_{1}}} & \sum_{i_{2}=1}^{N_{a_{2}}} \int_{0}^{1} \mathrm{~d} s_{1} \mathrm{~d} s_{2} \\
& \times\left(2 \delta_{a_{1}, a_{2}}-1\right) W^{\prime}\left(\left|\boldsymbol{r}_{a_{1}, i_{1}}\left(s_{1}\right)-\boldsymbol{r}_{a_{1}, i_{2}}\left(s_{2}\right)\right|\right) .
\end{aligned}
$$

The total Hamiltonian is denoted as $H^{\mathrm{T}}:=H^{\mathrm{W}}+H^{\mathrm{EV}}+H^{\mathrm{C}}$.

\subsubsection{Permanent crosslinks and partition function}

The most interesting properties of the polymer blend are due to the possible introduction of crosslinks, leading to a polymer network that inseparably connects $A$ 
and $B$ chains and that eventually drives the transition from a liquid to an amorphous solid. We consider the case of the randomly introduced chemical crosslinks, covalent bonds with a high binding energy. Like the intramolecular bonds in the individual polymer chains, they are permanent on the time scale of demixing processes and consequently treated as quenched randomness. Physical gelation, the association of macromolecules via temporary bonds of much lower energy, such as hydrogen bonds, is a likewise interesting problem, yet it is not part of the discussion here.

Following Goldbart et al. [77], the crosslinks are modelled as hard constraints, forcing two randomly chosen monomers to occupy exactly the same location. Regardless of how it is generated, a specific realisation of crosslinks is defined by a set of index pairs $\left\{\left(a_{e}, i_{e}, s_{e}, a_{e}^{\prime}, i_{e}^{\prime}, s_{e}^{\prime}\right)\right\}_{e=1}^{M}$ indicating that link number $e$ connects monomer on site $s_{e}$ of chain $i_{e}$ of type $a_{e}$ to monomer $s_{e}^{\prime}$ on chain $i_{e}^{\prime}$ of type $a_{e}^{\prime}$, where $M$ is the total number of crosslinks. Hence only those configurations are admitted that satisfy

$$
\boldsymbol{r}_{a_{e}, i_{e}}\left(s_{e}\right)=\boldsymbol{r}_{a_{e}^{\prime}, i_{e}^{\prime}}\left(s_{e}^{\prime}\right) \text { for } \quad e=1, \ldots, M
$$

The constraints shall be purely geometric, so that the introduction of crosslinks leaves the relative statistical weights of the allowed configurations, at least a priori, unaffected, i.e. proportional to $\exp \left(-\beta H^{\mathrm{E}}\right)$. This assumption is not fully correct, though:

First, a sufficiently high degree of crosslinking induces the transition from a liquid to an amorphous solid, in which the monomers are partly localised. The translational and rotational symmetries are spontaneously broken, only a fraction of the states really assumes a nonzero weight. Yet, the symmetry breaking technically occurs only at the stage of minimising the free energy, the partition function still contains all the configurations that meet condition (3.13).

Second, the introduction of crosslinks gives rise to closed loops, and a complete definition of the crosslinked would have to comprise not only the identities of the bonded monomer pairs, but also the topology of the loops: Typically, some loops will be interlocked, hence only a fraction of the configurations satisfying (3.13) is accessible in reality. (Mere entanglements of unconnected chains slow down but do not inhibit transitions between different parts of the configuration space.) In principle, the broken ergodicity should be taken into account in the partition function. However, since no analytical, microscopic way of treating the topological constraints properly is known so far, they are treated as annealed. This widely used approximation corresponds to a network of phantom chains that may freely pass through each other and themselves, although overlapping is forbidden by the excluded volume interaction. In the vicinity of the gelation transition, which our analysis focuses on, the number of crosslinks per macromolecule is of the order one, so the density of loops is low and the number and influence of interlockings is small.

We define the partition function of the crosslinked blend, relative to that of a 
melt of uncrosslinked, non-interacting chains, as

$$
\begin{aligned}
\tilde{Z}\left(\left\{\left(a_{e}, i_{e}, s_{e}, a_{e}^{\prime}, i_{e}^{\prime}, s_{e}^{\prime}\right)\right\}_{e=1}^{M}\right):= \\
\left\langle\mathrm{e}^{-\beta\left(H^{\mathrm{EV}}+H^{\mathrm{C}}\right)} \prod_{e=1}^{M} \delta\left(\boldsymbol{r}_{a_{e}, i_{e}}\left(s_{e}\right)-\boldsymbol{r}_{a_{e}^{\prime}, i_{e}^{\prime}}\left(s_{e}^{\prime}\right)\right)\right\rangle^{\mathrm{W}},
\end{aligned}
$$

where

$$
\left\langle\mathcal{O}\left[\left\{\boldsymbol{r}_{a, i}(s)\right\}\right]\right\rangle^{\mathrm{W}}:=\int \mathcal{D} \boldsymbol{r}_{a, i}(s) \mathcal{O}\left[\left\{\boldsymbol{r}_{a, i}(s)\right\}\right] \mathrm{e}^{-\beta H^{W}} / \int \mathcal{D} \boldsymbol{r}_{a, i}(s) \mathrm{e}^{-\beta H^{W}}
$$

denotes the expectation value of an observable $\mathcal{O}$ in the uncrosslinked, noninteracting melt, i.e. with respect to the Wiener Hamiltonian. The integral $\int \mathcal{D} \boldsymbol{r}_{a, i}(s)$ is the path integral over all possible configurations of all chains.

The tilde shall indicate that eq. (3.14) is not entirely the physical partition function, insofar as the Gibbsian factors for the indistinguishability of identical chains are missing. Due to the randomness and the huge variety of clusters produced by crosslinking, the proper inclusion of indistinguishability is a very complicated task. However, as pointed out in detail by Goldbart et al. [77], the Gibbsian factors don't affect the relevant physical observables: For the canonical ensemble with a specific realisation of crosslinks, these factors are constant and cancel out in thermal average, since they occur likewise in the statistical weight and in the normalisation. In the following, eq. (3.14) will therefore be referred to as "the" partition function.

\subsubsection{Thermal averages in the presence of crosslinks}

For convenience, we define a notation for the thermal average in the presence of a particular set

$$
\mathcal{C}:=\left\{\left(a_{e}, i_{e}, s_{e}, a_{e}^{\prime}, i_{e}^{\prime}, s_{e}^{\prime}\right)\right\}_{e=1}^{M}
$$

of crosslinks:

$$
\left\langle\mathcal{O}\left[\left\{\boldsymbol{r}_{a, i}(s)\right\}\right]\right\rangle_{\mathcal{C}}:=\frac{\left\langle\prod_{e=1}^{M} \delta\left(\boldsymbol{r}_{a_{e}, i_{e}}\left(s_{e}\right)-\boldsymbol{r}_{a_{e}^{\prime}, i_{e}^{\prime}}\left(s_{e}^{\prime}\right)\right) \mathcal{O}\left[\left\{\boldsymbol{r}_{a, i}(s)\right\}\right]\right\rangle^{\mathrm{T}}}{\left\langle\prod_{e=1}^{M} \delta\left(\boldsymbol{r}_{a_{e}, i_{e}}\left(s_{e}\right)-\boldsymbol{r}_{a_{e}^{\prime}, i_{e}^{\prime}}\left(s_{e}^{\prime}\right)\right)\right\rangle^{\mathrm{T}}},
$$

for arbitrary observables $\mathcal{O}$. The average $\langle\ldots\rangle^{\mathrm{T}}$ is taken with respect to the total Hamiltonian $H^{\mathrm{T}}$.

\subsubsection{Disorder averages and Deam-Edwards distribution}

For the characterisation of crosslinked polymer blends, the consideration of a sample with a specific realisation of crosslinks is either insufficient or infeasible: While the properties of a small sample are not representative, those of a macroscopically 
large one, though typical by virtue of self-averaging, are practically impossible to compute. Thus, the physical quantities must be averaged explicitly over the quenched disorder of crosslinks.

As a matter of course, the according probability distribution depends on the way the crosslinks are introduced. A very natural and convenient choice was proposed by Deam and Edwards [80], based on two ideas: First, only those pairs of monomers have a chance to be crosslinked that are nearby in the native melt. In other words, the more compatible the set of disorder is with the primary melt, the more likely its implementation is. Second, in real experiments the number of crosslinks fluctuates from sample to sample, being controlled by a chemical potential. Hence, for the probability of a given set $\mathcal{C}$ of $M$ crosslinks, we choose the Deam-Edwards distribution

$$
\mathbb{P}_{M}(\mathcal{C})=\mathcal{N}^{-1} \cdot \frac{(\mu V / 2 N)^{M}}{M !} \tilde{Z}_{\mathrm{p}}(\mathcal{C}) .
$$

The partition function $\tilde{Z}_{\mathrm{p}}$ measures the compatibility of the constraints with the native melt by counting the number of compliant states; the factor $\mu V / 2 N$ can be interpreted as the fugacity of the crosslinks. As indicated by the subscript, $\tilde{Z}_{\mathrm{p}}$ reflects the situation prior to crosslinking and has to be computed with the according values of $\lambda=\lambda_{\mathrm{p}}$ and $\chi=\chi_{\mathrm{p}}$. To avoid unnecessary complications, the crosslinked monomer pairs will be expressed as tuples rather than sets in the following 1 , so that differently ordered but otherwise identical crosslink realisations are counted separately, see eq. (3.19). The over-counting is compensated by the factor of $M$ ! in the denominator.

The disorder average of an arbitrary observable $\mathcal{O}$ with respect to $\mathbb{P}_{M}$ reads

$$
\begin{aligned}
{\left[\mathcal{O}\left(\left\{\boldsymbol{r}_{a, i}(s)\right\}\right)\right]:=\sum_{M=0}^{\infty} } & \left(\sum_{a_{1}, a_{1}^{\prime}} \sum_{i_{1}, i_{1}^{\prime}} \int_{0}^{1} \mathrm{~d} s_{1} \int_{0}^{1} \mathrm{~d} s_{1}^{\prime} \cdots \sum_{a_{M}, a_{M}^{\prime}} \sum_{i_{M}, i_{M}^{\prime}} \int_{0}^{1} \mathrm{~d} s_{M} \int_{0}^{1} \mathrm{~d} s_{M}^{\prime}\right. \\
& \left.\times \mathbb{P}_{M}\left(\left\{\left(a_{e}, i_{e}, s_{e}, a_{e}^{\prime}, i_{e}^{\prime}, s_{e}^{\prime}\right)\right\}_{e=1}^{M}\right) \mathcal{O}\left(\left\{\boldsymbol{r}_{a, i}(s)\right\}\right)\right),
\end{aligned}
$$

hence the normalisation constant $\mathcal{N}$ in eq. (3.18) is given by

$$
\mathcal{N}=\left\langle\mathrm{e}^{-\beta\left(H_{0}^{\mathrm{EV}}+H_{0}^{\mathrm{C}}\right)} \cdot \exp \left\{\frac{\mu V}{2 N} \sum_{a, a^{\prime}, i, i^{\prime}} \int_{0}^{1} \mathrm{~d} s \mathrm{~d} s^{\prime} \delta\left(\boldsymbol{r}_{a, i}(s)-\boldsymbol{r}_{a^{\prime}, i^{\prime}}\left(s^{\prime}\right)\right)\right\}\right\rangle_{0}^{\mathrm{W}}
$$

The Hamiltonians $H_{0}^{\mathrm{EV}}$ and $H_{0}^{\mathrm{C}}$ in the average $\langle\ldots\rangle_{0}^{\mathrm{W}}$ in eq. (3.20) have to be evaluated for the conditions at the instant of crosslinking, $\lambda_{\mathrm{p}}$ and $\chi_{\mathrm{p}}$.

Since our crosslinking procedure depends on the state of the system at the time of preparation, the final gel will permanently remember this state to a certain extent, even if the external conditions are changed afterwards. In other words,

\footnotetext{
${ }^{1}$ Nonetheless we will stick to curly braces in the notation.
} 
the properties of the sample depend on the history of preparation, which can become arbitrarily complicated if external conditions change during preparation or between multiple preparation steps. Hence it is important to stress that by using the Deam-Edwards distribution (3.18), we restrict the discussion to systems prepared by an instantaneous, one-step crosslinking process. The final sample is characterised by the external conditions, essentially the values of $\chi$, at the times of crosslinking and measurement.

\subsection{Order parameters}

The examination of crosslinked homopolymer blends requires two order parameters, one to detect gelation and one to detect phase separation of the two polymer species, which are considered in the following.

\subsubsection{Gelation order parameter}

To detect the transition from the liquid to the amorphous solid state, we use an order parameter initially proposed in the context of spin glasses by Edwards and Anderson [81], adapted to polymer gelation by Goldbart et al. [77]:

$$
\bar{Q}_{\boldsymbol{k}_{1} \ldots \boldsymbol{k}_{g}}:=\sum_{a=A, B} \sum_{i=1}^{N_{a}} \int_{0}^{1} \mathrm{~d} s\left\langle\exp \left(\mathrm{i} \boldsymbol{k}_{1} \boldsymbol{r}_{a, i}(s)\right)\right\rangle_{\mathcal{C}} \ldots\left\langle\exp \left(\mathrm{i} \boldsymbol{k}_{g} \boldsymbol{r}_{a, i}(s)\right)\right\rangle_{\mathcal{C}}
$$

with $g=1,2, \ldots$ and $\boldsymbol{k}_{1}, \ldots, \boldsymbol{k}_{g} \neq \mathbf{0}$. As defined in eq. (3.17), the symbol $\langle\ldots\rangle_{\mathcal{C}}$ denotes the thermal average in presence of a particular realisation $\mathcal{C}$ of crosslinks, i.e. prior to disorder averaging. For $g=1$, eq. (3.21) is the thermal average of

$$
Q_{\boldsymbol{k}}:=\sum_{a=A, B} \sum_{i=1}^{N_{a}} \int_{0}^{1} \mathrm{~d} s \mathrm{e}^{\mathrm{i} \boldsymbol{k r} \boldsymbol{r}_{a, i}(s)}
$$

that is, the monomer density in Fourier space.

To see how $\bar{Q}_{\boldsymbol{k}_{1} \ldots \boldsymbol{k}_{g}}$ discriminates between liquid and gel, we consider the thermal expectation value $\left\langle\exp \left(\mathrm{i} \boldsymbol{k} \boldsymbol{r}_{a, i}(s)\right)\right\rangle_{\mathcal{C}}$ of the density of a single monomer $(a, i, s)$ in Fourier space. In a liquid, the monomer is delocalised and explores the sample volume uniformly in the course of time, thus

$$
\left\langle\exp \left(\mathrm{i} \boldsymbol{k} \boldsymbol{r}_{a, i}(s)\right)\right\rangle_{\mathcal{C}}=0 \quad(\mathrm{a}, \mathrm{i}, \mathrm{s}) \text { delocalised }
$$

except for $\boldsymbol{k}=0$. In a solid, at least some of the monomers are localised. For such a monomer, $\left\langle\exp \left(\mathbf{i} \boldsymbol{k} \boldsymbol{r}_{a, i}(s)\right)\right\rangle_{\mathcal{C}}$ fails to vanish identically and can be written as

$$
\left\langle\exp \left(\mathrm{i} \boldsymbol{k} \boldsymbol{r}_{a, i}(s)\right)\right\rangle_{\mathcal{C}}=\mathrm{e}^{\mathrm{i} \boldsymbol{k} \boldsymbol{c}_{a, i}(\boldsymbol{s})} \bar{\rho}_{a, i, s}(\boldsymbol{k}) \quad(\mathrm{a}, \mathrm{i}, \mathrm{s}) \text { localised }
$$


where $\boldsymbol{c}_{a, i}(s)$ is the centre of its localisation, and $\bar{\rho}_{a, i, s}(\boldsymbol{k})$ denotes the Fourier transformed shape of the localised density, shifted to the origin. Insertion into (3.21) yields

$$
\bar{Q}_{\boldsymbol{k}_{1} \ldots \boldsymbol{k}_{g}}=\sum_{a=A, B} \sum_{i=1}^{N_{a}} \int_{0}^{1} \mathrm{~d} s \bar{\rho}_{a, i, s}\left(\boldsymbol{k}_{\mathbf{1}}\right) \ldots \bar{\rho}_{a, i, s}\left(\boldsymbol{k}_{\boldsymbol{g}}\right) \mathrm{e}^{\mathrm{i}\left(\boldsymbol{k}_{1}+\ldots+\boldsymbol{k}_{g}\right) \boldsymbol{c}_{a, i}(s)}
$$

where the integral and the sums are understood to include just the localised monomers.

Although the average total density $\bar{Q}_{\boldsymbol{k}}$ might seem already sufficient to detect gelation, this is in fact not the case [77]: In contrast to a globular or crystalline solid, the amorphous solid generated by crosslinking is macroscopically translationally (and rotationally) invariant (MTI), since the localisation centres $\boldsymbol{c}_{a, i}(s)$ are distributed randomly and homogeneously over the sample. Therefore, the phase factors in eq. (3.25) interfere destructively, and $\bar{Q}_{\boldsymbol{k}_{1} \ldots \boldsymbol{k}_{g}} \neq 0$ only if $\boldsymbol{k}_{1}+\ldots+\boldsymbol{k}_{g}=\mathbf{0}$, which is only possible for $g \geq 2$.

To summarise, the $g \geq 2$ values of the order parameter (3.21) can distinguish between the liquid state and the amorphous solid,

$$
\left.\bar{Q}_{\boldsymbol{k}_{1} \ldots \boldsymbol{k}_{g}}\right|_{\boldsymbol{k}_{1}+\ldots+\boldsymbol{k}_{g}=0} \begin{cases}=0, & \text { liquid } \\ \neq 0, & \text { gel. }\end{cases}
$$

Globular and crystalline states go beyond the scope of this thesis; their signature in the order parameter is discussed in [77].

Order parameter hypothesis Following [76, 77], we develop a hypothesis for the order parameter $\bar{Q}_{\boldsymbol{k}_{1} \ldots \boldsymbol{k}_{g}}$, which we shall use in the variational determination of the equilibrium state of the gel. In general, only a fraction $f_{\text {gel }}$ of monomers, the gel fraction, will be localised, while the remaining sol fraction $\left(1-f_{\text {gel }}\right)$ stays delocalised. To describe the gel fraction, we adopt the generalised Einstein model, in analogy to Einstein's model of crystalline solids, in which every atom is localised independently by a harmonic potential. The monomers $\{(a, i, s)\}$ of the gel fraction are supposed to be independently localised about random points $\boldsymbol{c}_{a, i}(s)$ in isotropic harmonic potentials. The density of such a monomer is given by eq. (3.24) with $\bar{\rho}_{a, i, s}(\boldsymbol{k})=\exp \left(-\boldsymbol{k}^{2} / 2 \tau_{a, i}(s)\right)$ and an inverse squared localisation length of $\tau_{a, i}(s)$. These assumptions lead to

$$
\bar{Q}_{\boldsymbol{k}_{1} \ldots \boldsymbol{k}_{g}}=\sum_{a=A, B} \sum_{i=1}^{N_{a}} \int_{0}^{1} \mathrm{~d} s \mathrm{e}^{\mathrm{i}\left(\boldsymbol{k}_{1}+\ldots+\boldsymbol{k}_{g}\right) \boldsymbol{c}_{a, i}(s)} \cdot \mathrm{e}^{-\left(\boldsymbol{k}_{1}^{2}+\ldots+\boldsymbol{k}_{g}^{2}\right) / 2 \tau_{a, i}(s)}
$$

with the sums and integrals just including the gel fraction. As pointed out before, the phase factors interfere destructively unless $\sum_{\gamma=1}^{g} \boldsymbol{k}_{\gamma}$ vanishes due to MTI, thus

$$
\bar{Q}_{\boldsymbol{k}_{1} \ldots \boldsymbol{k}_{g}}=\delta_{\boldsymbol{k}_{1}+\ldots+\boldsymbol{k}_{g}, \mathbf{0}} \cdot f_{\mathrm{gel}} \int_{0}^{\infty} \mathrm{d} \tau p(\tau) \exp \left(-\left(\boldsymbol{k}_{1}^{2}+\ldots+\boldsymbol{k}_{g}^{2}\right) / 2 \tau\right),
$$


where

$$
p(\tau)=\sum_{a=A, B} \sum_{i=1}^{N_{a}} \int_{0}^{1} \mathrm{~d} s\left\langle\delta\left(\tau-\tau_{a, i}(s)\right)\right\rangle_{\mathcal{C}}
$$

denotes the distribution of square localisation lengths; the sums in (3.29) again are restricted to the localised monomers. The distribution $p(\tau)$ and the gel fraction $f_{\text {gel }}$ will be determined in saddle-point approximation.

\subsubsection{Phase separation order parameter}

The separation of $A$ and $B$ chains or monomers can be recognised by the local imbalance of their concentrations,

$$
S_{\boldsymbol{k}}:=\sum_{i=1}^{N_{A}} \int_{0}^{1} \mathrm{~d} s \mathrm{e}^{-\mathrm{i} \boldsymbol{k} \boldsymbol{r}_{A, i}(s)}-\sum_{i=1}^{N_{B}} \int_{0}^{1} \mathrm{~d} s \mathrm{e}^{-\mathrm{i} \boldsymbol{k} \boldsymbol{r}_{B, i}(s)} .
$$

As in the case of random copolymers, eq. (3.30) will be referred to as the charge density, conceiving the two species as "charges" +1 and -1 . While in a homogeneously mixed liquid or gel, the disorder averaged equilibrium value $\left[\left\langle S_{\boldsymbol{k}}\right\rangle_{\mathcal{C}}\right]$ vanishes, it becomes nonzero in a phase separated state, reflecting the $A / B$ segregation pattern.

The structure factors

$$
S(\boldsymbol{k}):=\frac{1}{N}\left[\left\langle S_{\boldsymbol{k}} S_{-\boldsymbol{k}}\right\rangle_{\mathcal{C}}\right] \quad \text { and } \quad S_{\mathrm{gl}}(\boldsymbol{k}):=\frac{1}{N}\left[\left\langle S_{\boldsymbol{k}}\right\rangle_{\mathcal{C}}\left\langle S_{-\boldsymbol{k}}\right\rangle_{\mathcal{C}}\right]
$$

describe the disorder averaged charge correlations. While $S_{\mathrm{gl}}$ measures the time persistent part, $S$ measures the sum of both, static and volatile correlations. Physically, the glassy correlation function $S_{\mathrm{gl}}$ detects the charge correlations that originate from thermal fluctuations that have been frozen-in at the instant of preparation, and from the disorder due to the randomness of the crosslinks. The scattering structure factor $S$ additionally measures the thermal fluctuations at the time of measurement. From a mathematical point of view it might seem more natural to consider, besides $S_{\mathrm{gl}}$, the connected correlation or variance

$$
S_{\mathrm{th}}(\boldsymbol{k}):=\frac{1}{N}\left[\left\langle S_{\boldsymbol{k}} S_{-\boldsymbol{k}}\right\rangle_{\mathcal{C}}-\left\langle S_{\boldsymbol{k}}\right\rangle_{\mathcal{C}}\left\langle S_{-\boldsymbol{k}}\right\rangle_{\mathcal{C}}\right]=S(\boldsymbol{k})-S_{\mathrm{gl}}(\boldsymbol{k})
$$

instead of $S$; however, the latter is more directly related to scattering experiments (see below).

\subsubsection{Relation to scattering experiments}

The gelation order parameter (3.21), at least for $g=2$, and the structure factors (3.30) are closely related to scattering experiments (for an introduction to 
neutron and X-ray scattering in polymers, see, for example, [82-84]). The differential scattering cross section of a sample containing $N$ scattering centres is given by

$$
\frac{1}{N} \frac{\mathrm{d}^{2} \sigma}{\mathrm{d} \Omega \mathrm{d} E}=\frac{k_{\mathrm{o}}}{k_{\mathrm{i}}} S(\boldsymbol{k}, \omega)
$$

where $k_{\mathrm{i}}$ and $k_{\mathrm{o}}$ denote the moduli of the ingoing and outgoing wavevector. The momentum and energy transfers are $\boldsymbol{k}:=\boldsymbol{k}_{\mathrm{o}}-\boldsymbol{k}_{\mathrm{i}}$ and $\hbar \omega$. In the first Born approximation, the dynamic structure factor or scattering function is given by

$$
S(\boldsymbol{k}, \omega)=\frac{1}{2 \pi \hbar N} \int \mathrm{d} t\left\langle\sum_{i=1}^{N} b_{i}^{*} \mathrm{e}^{-\mathrm{i} \boldsymbol{k} \boldsymbol{r}_{i}(0)} \sum_{j=1}^{N} b_{j} \mathrm{e}^{\mathrm{i} \boldsymbol{k} \boldsymbol{r}_{j}(t)}\right\rangle \mathrm{e}^{-\mathrm{i} \omega t},
$$

where $\boldsymbol{r}_{i}(t)$ and $b_{i}$ denote the position at time $t$ and the scattering length (or atomic scattering factor) of centre number $i$, respectively. (The scattering function $S(\boldsymbol{k})$ should not be confused with the charge density $S_{\boldsymbol{k}}$.) The type of radiation used in the experiment determines whether the measurement focuses on static or dynamic properties: To achieve a sufficiently high spatial resolution, i.e. wavelengths comparable to interatomic distances, the energy of X-rays must be of the order of $10 \mathrm{MeV}$. Hence modulations due to atomic motion, having a typical energy smaller by orders of magnitude, can't be resolved. As the momentum transfer $k$ is small compared to the wavenumbers of the incident and the scattered light, we can drop the factor $k_{\mathrm{o}} / k_{\mathrm{i}} \approx 1$ in eq. (3.33), so that the integral cross section is given by

$$
\left(\frac{\mathrm{d} \sigma}{\mathrm{d} \Omega}\right)^{\text {(X-ray) }}=\int_{0}^{\infty} \mathrm{d}(\hbar \omega) \frac{\mathrm{d}^{2} \sigma}{\mathrm{d} \Omega \mathrm{d} E}=\left\langle\sum_{i=1}^{N} b_{i}^{*} \mathrm{e}^{-\mathrm{i} \boldsymbol{k} \boldsymbol{r}_{i}} \sum_{j=1}^{N} b_{j} \mathrm{e}^{\mathrm{i} \boldsymbol{k} \boldsymbol{r}_{j}}\right\rangle .
$$

The energy of neutrons with suitable wavelengths, on the other hand, are comparable to the thermal energy, leading to a considerable energy transfer caused by the interaction with atomic motion. In particular, it is possible to measure the elastic scattering function characterised by $\omega=0$,

$$
\left(\frac{\mathrm{d} \sigma}{\mathrm{d} \Omega}\right)^{(\mathrm{el})}=\left.\frac{\mathrm{d}^{2} \sigma}{\mathrm{d} \Omega \mathrm{d} E}\right|_{\omega=0} \propto \lim _{t \rightarrow \infty}\left\langle\sum_{i=1}^{N} b_{i}^{*} \mathrm{e}^{-\mathrm{i} \boldsymbol{k} \boldsymbol{r}_{i}(0)} \sum_{j=1}^{N} b_{j} \mathrm{e}^{\mathrm{i} \boldsymbol{k} \boldsymbol{r}_{j}(t)}\right\rangle ;
$$

in the last step we have used that in the integral over all times $t$, the weight of small $t$ is negligible. Because of the decay of thermal correlations, the thermal average in eq. (3.36) factorises for large $t$, hence

$$
\left(\frac{\mathrm{d} \sigma}{\mathrm{d} \Omega}\right)^{(\mathrm{e} \mathrm{l})}=\left\langle\sum_{i=1}^{N} b_{i}^{*} \mathrm{e}^{-\mathrm{i} \boldsymbol{k} \boldsymbol{r}_{i}}\right\rangle\left\langle\sum_{j=1}^{N} b_{j} \mathrm{e}^{\mathrm{i} \boldsymbol{k} \boldsymbol{r}_{j}}\right\rangle,
$$

yielding the persistent part of the correlations in the sample. Eq. (3.37) can be split into the so-called coherent scattering function of the self-interference of single 
atoms $(i=j)$ and the incoherent scattering function of the interference of distinct atoms $(i \neq j)$,

$$
\left(\frac{\mathrm{d} \sigma}{\mathrm{d} \Omega}\right)^{(\mathrm{el})}=\underbrace{\sum_{i=1}^{N}\left|b_{i}\right|^{2}\left|\left\langle\mathrm{e}^{\mathrm{i} \boldsymbol{k} \boldsymbol{r}_{i}}\right\rangle\right|^{2}}_{=:\left(\frac{\mathrm{d} \sigma}{\mathrm{d} \Omega}\right)_{\text {coh }}^{(\mathrm{el})}}+\underbrace{\sum_{i \neq j} b_{i}^{*} b_{j}\left\langle\mathrm{e}^{-\mathrm{i} \boldsymbol{k} \boldsymbol{r}_{i}}\right\rangle\left\langle\mathrm{e}^{\mathrm{i} \boldsymbol{k} \boldsymbol{r}_{j}}\right\rangle}_{=:\left(\frac{\mathrm{d} \sigma}{\mathrm{d} \Omega}\right)_{\text {incoh }}^{(\mathrm{el})}} .
$$

To apply these results to our polymer blend, we identify the scattering centres with the monomers and assume that all monomers of a given species reveal the same (real) scattering length, i.e. $\left(b_{0} \pm b_{1}\right)$ for $A$ and $B$ monomers, respectively, so that

$$
\begin{aligned}
& \left(\frac{\mathrm{d} \sigma}{\mathrm{d} \Omega}\right)^{(\mathrm{X}-\text { ray })}=b_{0}^{2}\left\langle\left|Q_{\boldsymbol{k}}\right|^{2}\right\rangle_{\mathcal{C}}+2 b_{0} b_{1} \operatorname{Re}\left\langle Q_{-\boldsymbol{k}} S_{\boldsymbol{k}}\right\rangle_{\mathcal{C}}+b_{1}^{2}\left\langle\left|S_{\boldsymbol{k}}\right|^{2}\right\rangle_{\mathcal{C}} \\
& \left(\frac{\mathrm{d} \sigma}{\mathrm{d} \Omega}\right)^{(\mathrm{el})}=b_{0}^{2}\left|\left\langle Q_{\boldsymbol{k}}\right\rangle_{\mathcal{C}}\right|^{2}+2 b_{0} b_{1} \operatorname{Re}\left(\left\langle Q_{-\boldsymbol{k}}\right\rangle_{\mathcal{C}}\left\langle S_{\boldsymbol{k}}\right\rangle_{\mathcal{C}}\right)+b_{1}^{2}\left|\left\langle S_{\boldsymbol{k}}\right\rangle_{\mathcal{C}}\right|^{2}
\end{aligned}
$$

and

$$
\left(\frac{\mathrm{d} \sigma}{\mathrm{d} \Omega}\right)_{\mathrm{coh}}^{(\mathrm{el})}=\left(b_{0}^{2}+b_{1}^{2}\right) \bar{Q}_{\boldsymbol{k},-\boldsymbol{k}}+b_{0} b_{1} \cdot \ldots
$$

The contributions of $Q$ and $S$ can, in principle, be separated varying $b_{0}$ and $b_{1}$ by means of isotopic substitution [82] in neutron scattering or heavy atom labelling in X-ray diffraction [85]: For instance, the incoherent cross section of deuterium is a factor of 40 smaller than that of hydrogen. Indeed, in both, [49] and [50], one of the two homopolymer species was deuterated. In this case, practically $b_{0} \approx 0$, so that, apart from numerical factors,

$$
\frac{1}{N}\left(\frac{\mathrm{d} \sigma}{\mathrm{d} \Omega}\right)_{\mathrm{coh}}^{(\mathrm{el})} \approx \frac{1}{N}\left[\bar{Q}_{\boldsymbol{k},-\boldsymbol{k}}\right]
$$

and

$$
\frac{1}{N}\left(\frac{\mathrm{d} \sigma}{\mathrm{d} \Omega}\right)^{(\mathrm{el})} \approx \frac{1}{N}\left[\left|\left\langle S_{\boldsymbol{k}}\right\rangle_{\mathcal{C}}\right|^{2}\right]=S_{\mathrm{gl}}(\boldsymbol{k})
$$

after disorder averaging. The according expression for the X-ray cross section,

$$
\frac{1}{N}\left(\frac{\mathrm{d} \sigma}{\mathrm{d} \Omega}\right)^{(\text {X-ray })} \approx \frac{1}{N}\left[\left\langle\left|S_{\boldsymbol{k}}\right|^{2}\right\rangle_{\mathcal{C}}\right]=S(\boldsymbol{k})
$$

can be obtained by setting $b_{0}=0$ by heavy atom labelling. Roughly speaking, we may refer to $S(\boldsymbol{k})$ and $S_{\mathrm{gl}}(\boldsymbol{k})$ as the (incoherent) X-ray and neutron scattering function, respectively, and to $\bar{Q}_{\boldsymbol{k},-\boldsymbol{k}}$ as the coherent neutron scattering function.

It should be noted that deuteration may have a significant effect on the miscibility of polymers; for example, critical mixtures of deuterated and protonated polybutadiene become immiscible below $99.2^{\circ} \mathrm{C}$ [50]. Thus in practice, the distinction of the contributions of the monomer density and the charge density may be difficult in some cases. 


\subsection{Derivation of an effective free energy}

The aim of this section is to derive an effective free energy of the crosslinked homopolymer blends modelled in section 3.2 . We shall use the dimensionless representation, taking the distance dependence of the excluded volume and incompatibility interactions to be delta-shaped, i.e. $U(r)=W(r)=\delta(r)$.

As the first step, we perform the disorder average of the free energy using the replica trick, which effectively leads to the partition function of $(n+1)$ copies of the system. This partition function is subsequently expressed as the integral over an effective partition function that depends on fields closely related to the order parameters $\bar{Q}_{\boldsymbol{k}_{1} \ldots \boldsymbol{k}_{g}}$ and $S_{\boldsymbol{k}}$ defined in section 3.3 ; the microscopic variables $\left\{\boldsymbol{r}_{a, i}(s)\right\}$ are integrated out. The corresponding effective free energy will allow us to determine, for instance, the most probable values of the order parameters which can be interpreted, within mean-field approximation, as the equilibrium state of the system.

\subsubsection{Disorder averaged free energy}

In this section, we formally compute the disorder average of the free energy, i.e. the average of the logarithm of the partition function (3.14). With the replica trick described in appendix A.1, we express the mean free energy as

$$
F:=-[\ln \tilde{Z}(\mathcal{C})]=\lim _{n \rightarrow 0} \frac{1-Z_{n}}{n},
$$

where

$$
Z_{n}:=\left[(\tilde{Z}(\mathcal{C}))^{n}\right]
$$

denotes the disorder average of the $n$th power of the partition function. The DeamEdwards distribution (3.18) produces an $(n+1)$ th factor of the partition function in the disorder averaged free energy,

$$
\begin{aligned}
Z_{n}=\frac{1}{\mathcal{N}} \sum_{M=0}^{\infty} & \frac{(\mu V / 2 N)^{M}}{M !} \sum_{a_{1}, a_{1}^{\prime}} \sum_{i_{1}, i_{1}^{\prime}} \int_{0}^{1} \mathrm{~d} s_{1} \mathrm{~d} s_{1}^{\prime} \cdots \sum_{a_{M}, a_{M}^{\prime}} \sum_{i_{M}, i_{M}^{\prime}} \int_{0}^{1} \mathrm{~d} s_{M} \mathrm{~d} s_{M}^{\prime} \\
& \times \tilde{Z}_{\mathrm{p}}\left(\left\{\left(a_{e}, i_{e}, s_{e}, a_{e}^{\prime}, i_{e}^{\prime}, s_{e}^{\prime}\right)\right\}\right)\left(\tilde{Z}\left(\left\{\left(a_{e}, i_{e}, s_{e}, a_{e}^{\prime}, i_{e}^{\prime}, s_{e}^{\prime}\right)\right\}\right)\right)^{n} .
\end{aligned}
$$

As indicated by the subscript "p", the additional partition function must be evaluated at the conditions $\left(\chi_{\mathrm{p}}, \lambda_{\mathrm{p}}\right)$ of crosslinking. The normalisation constant $\mathcal{N}$ is defined in eq. (3.20). For nonnegative integer $n$, we can make the $n$th power of $\tilde{Z}$ explicit, and interpret $\tilde{Z}_{\mathrm{p}}(\mathcal{C})(\tilde{Z}(\mathcal{C}))^{n}$ as the partition function of a system comprising $(n+1)$ independent copies of the original system, the replicas, with the same realisation $\mathcal{C}$ of disorder in each copy.

We introduce the following notation for the replicated system: The monomer positions in the $\alpha$ th replica $(\alpha=0, \ldots, n)$ are denoted as $\left\{\boldsymbol{r}_{a, i}^{\alpha}(s)\right\}$, and the replicated 
total Hamiltonian is defined as $H_{n}^{\mathrm{T}}:=H_{n}^{\mathrm{W}}+H_{n}^{\mathrm{EV}}+H_{n}^{\mathrm{C}}$, where

$$
\begin{aligned}
H_{n}^{\mathrm{W}} & =\frac{1}{4} \sum_{\alpha=0}^{n} \sum_{a=A, B} \sum_{i=1}^{N_{a}} \int_{0}^{1} \mathrm{~d} s\left(\frac{\mathrm{d} \boldsymbol{r}_{a, i}^{\alpha}(s)}{\mathrm{d} s}\right)^{2} \\
H_{n}^{\mathrm{EV}} & =\frac{1}{2 \varrho_{0}} \sum_{\alpha=0}^{n} \lambda^{\alpha} \sum_{a_{1,2}=A, B} \sum_{i_{1}=1}^{N_{a_{1}}} \sum_{i_{2}=1}^{N_{a_{2}}} \int_{0}^{1} \mathrm{~d} s_{1} \mathrm{~d} s_{2} \delta\left(\boldsymbol{r}_{a_{1}, i_{1}}^{\alpha}\left(s_{1}\right)-\boldsymbol{r}_{a_{2}, i_{2}}^{\alpha}\left(s_{2}\right)\right)
\end{aligned}
$$

and

$$
\begin{aligned}
H_{n}^{\mathrm{C}}=-\frac{1}{2 \varrho_{0}} \sum_{\alpha=0}^{n} \chi^{\alpha} \sum_{a_{1,2}=A, B} \sum_{i_{1}=1}^{N_{a_{1}}} \sum_{i_{2}=1}^{N_{a_{2}}} \int_{0}^{1} \mathrm{~d} s_{1} \mathrm{~d} s_{2} \\
\times\left(2 \delta_{a_{1}, a_{2}}-1\right) \delta\left(\boldsymbol{r}_{a_{1}, i_{1}}^{\alpha}\left(s_{1}\right)-\boldsymbol{r}_{a_{2}, i_{2}}^{\alpha}\left(s_{2}\right)\right) .
\end{aligned}
$$

The respective expectation values are denoted as $\langle\ldots\rangle_{n}^{\mathrm{T}},\langle\ldots\rangle_{n}^{\mathrm{W}}$ and so on; for $n=$ 0 , this definition coincides with the notation used in eq. (3.20). The values of $\lambda$ and $\chi$ are chosen according to

$$
\lambda^{\alpha}=\left\{\begin{array}{ll}
\lambda_{\mathrm{p}}, & \text { if } \alpha=0, \\
\lambda_{\mathrm{m}}, & \text { otherwise },
\end{array} \quad \text { and } \quad \chi^{\alpha}= \begin{cases}\chi_{\mathrm{p}}, & \text { if } \alpha=0 \\
\chi_{\mathrm{m}}, & \text { otherwise }\end{cases}\right.
$$

to account for the potentially different states of the preparation ensemble, described by $\tilde{Z}_{\mathrm{p}}$ in the zeroth replica, and the measurement ensemble, described by $\tilde{Z}$ in the other replicas. It is useful to introduce some notation for the $(d \cdot(n+1))$ dimensional vectors $\hat{x}$ residing in the replicated space and the canonical basis $\left\{e_{\alpha}\right\}$ for the $(n+1)$-dimensional replica space,

$$
\hat{x}:=\left(\boldsymbol{x}^{0}, \ldots, \boldsymbol{x}^{n}\right)=: \sum_{\alpha=0}^{n} \boldsymbol{x}^{\alpha} \otimes e_{\alpha} .
$$

With this notation and the explicit expression for $\tilde{Z}$ given in eq. (3.14) we obtain

$$
\begin{array}{r}
Z_{n}=\frac{1}{\mathcal{N}} \sum_{M=0}^{\infty} \frac{(\mu V / 2 N)^{M}}{M !} \sum_{a_{1}, a_{1}^{\prime}} \sum_{i_{1}, i_{1}^{\prime}} \int_{0}^{1} \mathrm{~d} s_{1} \mathrm{~d} s_{1}^{\prime} \cdots \sum_{a_{M}, a_{M}^{\prime}} \sum_{i_{M}, i_{M}^{\prime}} \int_{0}^{1} \mathrm{~d} s_{M} \mathrm{~d} s_{M}^{\prime} \\
\times\left\langle\mathrm{e}^{-H_{n}^{\mathrm{EV}}-H_{n}^{\mathrm{C}}} \prod_{e=1}^{M} \prod_{\alpha=0}^{n} \delta\left(\boldsymbol{r}_{a_{e}, i_{e}}^{\alpha}\left(s_{e}\right)-\boldsymbol{r}_{a_{e}^{\prime}, i_{e}^{\prime}}^{\alpha}\left(s_{e}^{\prime}\right)\right)\right\rangle_{n}^{\mathrm{W}}
\end{array}
$$

By changing the order of the average and the summations we express the products of delta functions as the exponential function of a single delta function, so that $Z_{n}$ becomes

$$
Z_{n}=\mathcal{Z}_{n} / \mathcal{Z}_{0}
$$


with the (rescaled) replicated partition function

$$
\mathcal{Z}_{n}:=\left\langle\mathrm{e}^{-H_{n}^{\mathrm{EV}}-H_{n}^{\mathrm{C}}} \exp \left(\frac{\mu V}{2 N} \sum_{a, a^{\prime}, i, i^{\prime}} \int_{0}^{1} \mathrm{~d} s \mathrm{~d} s^{\prime} \delta\left(\hat{r}_{a, i}(s)-\hat{r}_{a^{\prime}, i^{\prime}}\left(s^{\prime}\right)\right)\right)\right\rangle_{n}^{\mathrm{W}}
$$

In the last step, the product of delta functions has been replaced by a $(d \cdot(n+1))$ dimensional one in the replicated space with $\hat{r}_{a, i}(s):=\left(\boldsymbol{r}_{a, i}^{0}(s), \ldots, \boldsymbol{r}_{a, i}^{n}(s)\right)$.

\subsubsection{Effective free energy}

To faciliate the introduction of fields corresponding to the order parameters, we define shorthands for the replica versions of the order parameters $Q$ and $S$ of section 3.3 , yet without thermal averaging,

$$
\begin{gathered}
Q_{\boldsymbol{k}}^{\alpha}:=\sum_{a=A, B} \sum_{i=1}^{N_{a}} \int_{0}^{1} \mathrm{~d} s \mathrm{e}^{\mathrm{i} \boldsymbol{k} \boldsymbol{r}_{a, i}^{\alpha}(s)}, \quad Q_{\hat{k}}:=\sum_{a=A, B} \sum_{i=1}^{N_{a}} \int_{0}^{1} \mathrm{~d} s \mathrm{e}^{\mathrm{i} \hat{k} \hat{r}_{a, i}(s)}, \\
\quad \text { and } \\
S_{\boldsymbol{k}}^{\alpha}:=\sum_{i=1}^{N_{a}} \int_{0}^{1} \mathrm{~d} s\left(\mathrm{e}^{\mathrm{i} \boldsymbol{k} \boldsymbol{r}_{A, i}^{\alpha}(s)}-\mathrm{e}^{\mathrm{i} \boldsymbol{k} \boldsymbol{r}_{B, i}^{\alpha}(s)}\right) .
\end{gathered}
$$

We rewrite the Hamiltonians of the excluded volume and the incompatibility interaction as

$$
H_{n}^{\mathrm{EV}}=\frac{1}{2 N} \sum_{\alpha=0}^{n} \lambda^{\alpha} \sum_{\boldsymbol{k}}^{\prime} Q_{\boldsymbol{k}}^{\alpha} Q_{-\boldsymbol{k}}^{\alpha} \quad \text { and } \quad H_{n}^{\mathrm{C}}=-\frac{1}{2 N} \sum_{\alpha=0}^{n} \chi^{\alpha} \sum_{\boldsymbol{k}}^{\prime} S_{\boldsymbol{k}}^{\alpha} S_{-\boldsymbol{k}}^{\alpha}
$$

where $\sum_{k}^{\prime}$ denotes the sum over nonzero $\boldsymbol{k}$. The quasi-Hamiltonian that originates from the constraints due to the crosslinks can be recast similarly,

$$
\begin{aligned}
& -\frac{\mu V}{2 N} \sum_{a, a^{\prime}, i, i^{\prime}} \int_{0}^{1} \mathrm{~d} s \mathrm{~d} s^{\prime} \delta\left(\hat{r}_{a, i}(s)-\hat{r}_{a^{\prime}, i^{\prime}}\left(s^{\prime}\right)\right) \\
& =-\frac{\mu}{2 N V^{n}} \sum_{\hat{k}}^{\prime} Q_{\hat{k}} Q_{-\hat{k}}=-\frac{\mu}{2 N V^{n}}\left(\sum_{\alpha=0}^{n} \sum_{\boldsymbol{k}}^{\prime} Q_{k}^{\alpha} Q_{-k}^{\alpha}+\overline{\sum_{\hat{k}}} Q_{\hat{k}} Q_{-\hat{k}}\right),
\end{aligned}
$$

with $\sum_{\hat{k}}^{\prime}$ denoting the sum over nonzero $\hat{k}$. For technical reasons, this sum is split into the single replica sector of vectors $\hat{k}=\boldsymbol{x} \otimes e_{\alpha}$ with a nonzero entry $\boldsymbol{x}$ in just one replica, and the higher replica sector of $\hat{k}$ with nonzero entries in at least two replicas; the according sum is denoted as $\sum_{\hat{k}}$. The zero replica sector, i.e. terms with $\boldsymbol{k}=\mathbf{0}$ and $\hat{k}=\hat{0}$, has been omitted since it only contributes an additive constant to the free energy.

Now we can introduce the effective free energy by means of a Hubbard-Stratonovich transformation (see appendix A.2) for each $S_{\boldsymbol{k}}^{\alpha}, Q_{\boldsymbol{k}}^{\alpha}$ and $Q_{\hat{k}}$ with $\boldsymbol{k} \cdot \boldsymbol{n}>0$ 


\section{Crosslinked homopolymer blends}

or $\hat{k} \cdot \hat{n}>0$, respectively, where $\boldsymbol{n}$ and $\hat{n}$ are arbitrary nonzero vectors. The restriction to half-spaces is necessary due to the interdependence of the order parameters with opposite wavevectors: Corresponding to real-valued fields in real space, they are the complex conjugates of each other, and hence no independent degrees of freedom. The transformations yield

$$
Z_{n}=\mathcal{B}_{n} \cdot \frac{\int \mathcal{D}_{n} \Psi \mathcal{D}_{n} \Omega \overline{\mathcal{D}} \Omega \exp \left\{-n \mathcal{F}_{n}(\{\Psi, \Omega\})\right\}}{\int \mathcal{D} \psi \mathcal{D} \omega \exp \left\{-n \mathcal{F}_{\text {den }}(\{\psi, \omega\})\right\}},
$$

where the effective free energies per replica of the numerator, $\mathcal{F}_{n}$, and of the denominator, $\mathcal{F}_{\text {den }}$, are given by

$$
\begin{aligned}
n \mathcal{F}_{n}(\{\Psi, \Omega\}):= & \frac{N}{2} \sum_{\alpha=0}^{n} \sum_{\boldsymbol{k}}^{\prime}\left(\frac{1}{\chi^{\alpha}}\left|\Psi_{\boldsymbol{k}}^{\alpha}\right|^{2}+\frac{1}{\hat{\lambda}^{\alpha}}\left|\Omega_{\boldsymbol{k}}^{\alpha}\right|^{2}\right)+\frac{N}{2} \overline{\sum_{\hat{k}}} \frac{1}{V^{-n} \mu}\left|\Omega_{\hat{k}}\right|^{2} \\
& -\ln \left\langle\exp \left\{\sum_{\alpha=0}^{n} \sum_{\boldsymbol{k}}^{\prime}\left(\Psi_{\boldsymbol{k}}^{\alpha} S_{-\boldsymbol{k}}^{\alpha}+\mathrm{i} \Omega_{\boldsymbol{k}}^{\alpha} Q_{-\boldsymbol{k}}^{\alpha}\right)+\overline{\sum_{\hat{k}}} V^{-n} \mu \Omega_{\hat{k}} Q_{-\hat{k}}\right\}\right\rangle_{n}^{W}
\end{aligned}
$$

and

$$
\begin{aligned}
& n \mathcal{F}_{\text {den }}(\{\psi, \omega\}):=\frac{N}{2} \sum_{\boldsymbol{k}}^{\prime}(\left.\frac{1}{\chi_{\mathrm{p}}}\left|\psi_{\boldsymbol{k}}\right|^{2}+\frac{1}{\lambda_{\mathrm{p}}-\mu}\left|\omega_{\boldsymbol{k}}\right|^{2}\right) \\
&-\ln \left\langle\exp \left\{\sum_{\boldsymbol{k}}^{\prime}\left(\psi_{\boldsymbol{k}} S_{-\boldsymbol{k}}+\mathrm{i} \omega_{\boldsymbol{k}} Q_{-\boldsymbol{k}}\right)\right\}\right\rangle^{W},
\end{aligned}
$$

with $\tilde{\lambda}^{\alpha}:=\lambda^{\alpha}-V^{-n} \mu$. The constant

$$
\mathcal{B}_{n}=\exp \left(\frac{N}{2}\left(-n \lambda_{\mathrm{m}}+n q^{2} \chi_{\mathrm{m}}+\left((n+1) V^{-n}-1\right) \mu\right)\right)=1+\mathcal{O}(n)
$$

contains the contributions from the zero replica sector; it just yields an additive constant to the free energy and thus will be dropped. The integral runs over the real and imaginary parts of the fields; the integral measures are specified in appendix $\mathrm{D}$, eq. (D.68). The sums over the wavevectors are not restricted to half spaces; the fields are continued to the whole wavevector space via $\Omega_{-\boldsymbol{k}}^{\alpha}:=\left(\Omega_{\boldsymbol{k}}^{\alpha}\right)^{*}$ and so forth. With the effective free energies depending only linearly on $Q$ and $S$, the chains are decoupled, and the many-chain problem can be reduced to a single- 
chain problem:

$$
\begin{aligned}
& n \mathcal{F}_{n}(\{\Psi, \Omega\}):=\frac{N}{2} \sum_{\alpha, \boldsymbol{k}}^{\prime}\left(\frac{1}{\chi^{\alpha}}\left|\Psi_{\boldsymbol{k}}^{\alpha}\right|^{2}+\frac{1}{\hat{\lambda}^{\alpha}}\left|\Omega_{\boldsymbol{k}}^{\alpha}\right|^{2}\right)+\frac{N}{2} \overline{\sum_{\hat{k}}} \frac{1}{V^{-n} \mu}\left|\Omega_{\hat{k}}\right|^{2} \\
& -N_{A} \ln \left\langle\exp \left\{\sum_{\alpha, \boldsymbol{k}}^{\prime}\left(+\Psi_{\boldsymbol{k}}^{\alpha}+\mathrm{i} \Omega_{\boldsymbol{k}}^{\alpha}\right) \int_{0}^{1} \mathrm{~d} s \mathrm{e}^{-\mathrm{i} \boldsymbol{k} \boldsymbol{r}^{\alpha}(s)}+\overline{\sum_{\hat{k}}} \Omega_{\hat{k}} \int_{0}^{1} \mathrm{~d} s \mathrm{e}^{-\mathrm{i} \hat{k} \hat{r}(s)}\right\}\right\rangle_{n}^{W} \\
& -N_{B} \ln \left\langle\exp \left\{\sum_{\alpha, \boldsymbol{k}}^{\prime}\left(-\Psi_{\boldsymbol{k}}^{\alpha}+\mathrm{i} \Omega_{\boldsymbol{k}}^{\alpha}\right) \int_{0}^{1} \mathrm{~d} s \mathrm{e}^{-\mathrm{i} \boldsymbol{k} \boldsymbol{r}^{\alpha}(s)}+\overline{\sum_{\hat{k}}} \Omega_{\hat{k}} \int_{0}^{1} \mathrm{~d} s \mathrm{e}^{-\mathrm{i} \hat{k} \hat{r}(s)}\right\}\right\rangle_{n}^{W}
\end{aligned}
$$

with the shorthand $\sum_{\alpha, \boldsymbol{k}}^{\prime}:=\sum_{\alpha=0}^{n} \sum_{\boldsymbol{k}}^{\prime}$. The monomer positions of the remaining single chain are denoted as $\{\hat{r}(s)\}$, the according Wiener average is given by $\langle\cdots\rangle_{n}^{\mathrm{W}} \propto \int \mathcal{D} r(s) \cdots \exp \left(-\frac{1}{2} \int_{0}^{1} \mathrm{~d} s\left(\frac{\mathrm{d} \hat{r}(s)}{\mathrm{d} s}\right)^{2}\right)$.

\subsubsection{Expectation values of the order parameters}

We define the expectation value

$$
\langle\cdots\rangle_{n}^{\mathcal{F}}:=\frac{\int \mathcal{D}_{n} \Psi \mathcal{D}_{n} \Omega \overline{\mathcal{D}} \Omega \cdots \exp \left\{-n \mathcal{F}_{n}(\{\Psi, \Omega\})\right\}}{\int \mathcal{D}_{n} \Psi \mathcal{D}_{n} \Omega \overline{\mathcal{D}} \Omega \exp \left\{-n \mathcal{F}_{n}(\{\Psi, \Omega\})\right\}}
$$

with respect to the effective free energy in order to compute moments of the auxiliary fields $\Omega$ and $\Psi$ introduced by the Hubbard-Stratonovich transformations. These moments are connected to thermal averages of the moments of the order parameters $Q$ and $S$, which will permit us to determine the equilibrium state of the melt later. These relations are derived in appendix D. For the expectation values required to assess the stability of the liquid and the mixed state and to examine charge fluctuations, we obtain

$$
\begin{array}{rlrl}
{\left[\bar{Q}_{\boldsymbol{k}}\right]} & =\frac{\mathrm{i} N}{\lambda_{\mathrm{m}}-\mu} \lim _{n \rightarrow 0}\left\langle\Omega_{\boldsymbol{k}}^{\alpha}\right\rangle_{n}^{\mathcal{F}} & & \text { with } \alpha \geq 1, \\
{\left[\bar{Q}_{\boldsymbol{k}_{1} \ldots \boldsymbol{k}_{g}}\right]} & =\frac{N}{\mu} \lim _{n \rightarrow 0}\left\langle\Omega_{\hat{k}}\right\rangle_{n}^{\mathcal{F}} & \text { for } g \geq 2, \text { with } \hat{k}=\sum_{\gamma=1}^{g} \boldsymbol{k}_{\gamma} \otimes e_{\gamma}, \\
{\left[\left\langle S_{\boldsymbol{k}}\right\rangle_{\mathcal{C}}\right]} & =\frac{N}{\chi_{\mathrm{m}}} \lim _{n \rightarrow 0}\left\langle\Psi_{\boldsymbol{k}}^{\alpha}\right\rangle_{n}^{\mathcal{F}} & \text { with } \alpha \geq 1
\end{array}
$$

and

$$
\begin{array}{rr}
{\left[\left\langle S_{\boldsymbol{k}} S_{-\boldsymbol{k}}\right\rangle_{\mathcal{C}}\right]=\frac{N^{2}}{\left(\chi_{\mathrm{m}}\right)^{2}}\left(\lim _{n \rightarrow 0}\left\langle\Psi_{\boldsymbol{k}}^{\alpha} \Psi_{-\boldsymbol{k}}^{\alpha}\right\rangle_{n}^{\mathcal{F}}-\frac{\chi_{\mathrm{m}}}{N}\right)} & \text { with } \alpha \geq 1 \\
{\left[\left\langle S_{\boldsymbol{k}}\right\rangle_{\mathcal{C}}\left\langle S_{-\boldsymbol{k}}\right\rangle_{\mathcal{C}}\right]=\frac{N^{2}}{\left(\chi_{\mathrm{m}}\right)^{2}} \lim _{n \rightarrow 0}\left\langle\Psi_{\boldsymbol{k}}^{\alpha_{1}} \Psi_{-\boldsymbol{k}}^{\alpha_{2}}\right\rangle_{n}^{\mathcal{F}}} & \text { with } \alpha_{1,2} \geq 1 \text { and } \alpha_{1} \neq \alpha_{2} .
\end{array}
$$




\subsubsection{Shifted density field}

In asymmetric blends, in which there is an excess of either $A$ or $B$ chains, variations of the mass density, even without changing the local ratio of $A$ and $B$ monomers, imply variations of the charge density, because the average monomer charge

$$
q:=\left(N_{A}-N_{B}\right) / N
$$

is nonzero. Technically, this gives rise to a bilinear term in $\Psi_{\boldsymbol{k}}^{\alpha} \Omega_{-\boldsymbol{k}}^{\alpha}$ in the effective free energy (3.63), hidden in the logarithmic contributions. The coupling complicates tracing out the density field, which is useful since we are mainly interested in the phase separation behaviour of the system, in particular for low compressibility, where density fluctuations are small, anyway. In the incompressible limit, the bilinear coupling of $\Psi_{\boldsymbol{k}}^{\alpha}$ and $\Omega_{\boldsymbol{k}}^{\alpha}$ can be circumvented by rescaling the single-replica density field to "absorb" the asymmetry:

$$
\tilde{\Omega}_{k}^{\alpha}:=\Omega_{k}^{\alpha}-\mathrm{i} q \Psi_{k}^{\alpha}
$$

(An analogous rescaling was done in the discussion of random copolymer melts in section 2.5.2.) The effective free energy as a function of $\Psi_{\boldsymbol{k}}^{\alpha}, \Omega_{\hat{k}}$ and the new variable $\tilde{\Omega}_{\boldsymbol{k}}^{\alpha}$ reads

$$
\begin{aligned}
& n \mathcal{F}_{n}(\{\Psi, \tilde{\Omega}, \Omega\}):=\frac{N}{2} \overline{\sum_{\hat{k}}} \frac{1}{V^{-n} \mu}\left|\Omega_{\hat{k}}\right|^{2} \\
& \quad+\frac{N}{2} \sum_{\alpha, \boldsymbol{k}}^{\prime}\left(\left(\frac{1}{\chi^{\alpha}}-\frac{q^{2}}{\tilde{\lambda}^{\alpha}}\right)\left|\Psi_{\boldsymbol{k}}^{\alpha}\right|^{2}+\frac{\mathrm{i} q}{\tilde{\lambda}^{\alpha}}\left(\tilde{\Omega}_{\boldsymbol{k}}^{\alpha} \Psi_{-\boldsymbol{k}}^{\alpha}+\Psi_{\boldsymbol{k}}^{\alpha} \tilde{\Omega}_{-\boldsymbol{k}}^{\alpha}\right)+\frac{1}{\tilde{\lambda}^{\alpha}}\left|\tilde{\Omega}_{\boldsymbol{k}}^{\alpha}\right|^{2}\right) \\
& -N_{A} \ln \left\langle\exp \left\{\sum_{\alpha, \boldsymbol{k}}^{\prime}\left(\mathrm{i} \tilde{\Omega}_{\boldsymbol{k}}^{\alpha}+\frac{2 N_{B}}{N} \Psi_{\boldsymbol{k}}^{\alpha}\right) \int_{0}^{1} \mathrm{~d} s \mathrm{e}^{-\mathrm{i} \boldsymbol{k} \boldsymbol{r}^{\alpha}(s)}+\overline{\sum_{\hat{k}}} \Omega_{\hat{k}} \int_{0}^{1} \mathrm{~d} s \mathrm{e}^{-\mathrm{i} \hat{k} \hat{r}(s)}\right\}\right\rangle_{n}^{W} \\
& -N_{B} \ln \left\langle\exp \left\{\sum_{\alpha, \boldsymbol{k}}^{\prime}\left(\mathrm{i} \tilde{\Omega}_{\boldsymbol{k}}^{\alpha}-\frac{2 N_{A}}{N} \Psi_{\boldsymbol{k}}^{\alpha}\right) \int_{0}^{1} \mathrm{~d} s \mathrm{e}^{-\mathrm{i} \boldsymbol{k} \boldsymbol{r}^{\alpha}(s)}+\overline{\sum_{\hat{k}}} \Omega_{\hat{k}} \int_{0}^{1} \mathrm{~d} s \mathrm{e}^{-\mathrm{i} \hat{k} \hat{r}(s)}\right\}\right\rangle_{n}^{W} .
\end{aligned}
$$

The logarithmic contributions now produce a coupling only at higher order (see section 3.5.7), and the explicit bilinear term vanishes in the incompressible limit $\lambda_{\mathrm{p}}, \lambda_{\mathrm{m}} \rightarrow \infty$, where terms proportional to $1 / \tilde{\lambda}^{\alpha}$ can be neglected. In this limit, fluctuations of the physical monomer density are suppressed,

$$
\left[\bar{Q}_{\boldsymbol{k}}\right]=\frac{\mathrm{i} N}{\lambda_{\mathrm{m}}-\mu} \lim _{n \rightarrow 0}\left\langle\Omega_{\boldsymbol{k}}^{\alpha}\right\rangle_{n}^{\mathcal{F}}=\frac{\mathrm{i} N}{\lambda_{\mathrm{m}}-\mu} \lim _{n \rightarrow 0}\left\langle\tilde{\Omega}_{\boldsymbol{k}}^{\alpha}+\mathrm{i} \Psi_{\boldsymbol{k}}^{\alpha}\right\rangle_{n}^{\mathcal{F}} \stackrel{\lambda_{\mathrm{m}} \rightarrow \infty}{\longrightarrow} 0
$$

in contrast to the fluctuations of the corresponding field $\tilde{\Omega}$. Hence, nonzero values of $\tilde{\Omega}_{\boldsymbol{k}}^{\alpha}$ or $\Omega_{\boldsymbol{k}}^{\alpha}$ are not in contradiction with incompressibility. 


\subsection{Analysis}

In the following, we will analyse the free energy of the polymer blend. We start by determining the degree of stability of the mixed state before and after crosslinking, which permits us to draw a phase diagram in terms of the degree of crosslinking and the conditions at measurement. Subsequently, we discuss the relevant lengthscales in the system and how they rule the demixing correlations that show the preservation of pre-crosslinking fluctuations and give notice of a nearby microphase transition. Finally, we discuss the microphase separated state, considering different regular and random morphologies, and we study the effects of asymmetric composition and compressibility.

\subsubsection{Mean-field approximation}

As the effective free energy (3.63) is extensive, we may apply the saddle point approximation to eq. (3.59) in order to calculate expectation values of $\tilde{\Omega}, \Omega$ and $\Psi$. The integral in the numerator is replaced by the value of the integrand, evaluated at the saddle point $(\bar{\Psi}, \bar{\Omega}):=\left\{\bar{\Psi}_{\boldsymbol{k}}^{\alpha}, \bar{\Omega}_{\boldsymbol{k}}^{\alpha}, \bar{\Omega}_{\hat{k}}\right\}$ making it stationary with respect to variations of $\tilde{\Omega}, \Omega$ and $\Psi$; the denominator is treated analogously. Neglecting additive constants, we can express the free energy as

$$
F=\lim _{n \rightarrow 0}\left(\mathcal{F}_{n}(\bar{\Psi}, \overline{\tilde{\Omega}}, \bar{\Omega})-\mathcal{F}_{\operatorname{den}}(\bar{\omega}, \bar{\psi})\right) .
$$

This procedure corresponds to the mean-field level of approximation, where fluctuations are neglected, and the expectation values of the fields are approximated by their saddle point values

$$
\left\langle\Psi_{k}^{\alpha}\right\rangle_{n}^{\mathcal{F}} \approx \bar{\Psi}_{k}^{\alpha}, \quad\left\langle\tilde{\Omega}_{k}^{\alpha}\right\rangle_{n}^{\mathcal{F}} \approx \overline{\tilde{\Omega}}_{k}^{\alpha} \quad \text { and } \quad\left\langle\Omega_{\hat{k}}\right\rangle_{n}^{\mathcal{F}} \approx \bar{\Omega}_{\hat{k}}
$$

The saddle point values are the solutions of the stationarity conditions

$$
\left.\frac{\partial \mathcal{F}_{n}}{\partial \Psi_{-\boldsymbol{k}}^{\alpha}}\right|_{(\bar{\Psi}, \bar{\Omega})}=0,\left.\quad \frac{\partial \mathcal{F}_{n}}{\partial \tilde{\Omega}_{-\boldsymbol{k}}^{\alpha}}\right|_{(\bar{\Psi}, \bar{\Omega})}=0 \quad \text { and }\left.\quad \frac{\partial \mathcal{F}_{n}}{\partial \Omega_{-\hat{k}}}\right|_{(\bar{\Psi}, \bar{\Omega})}=0 .
$$

\subsubsection{Stability of the homogeneously mixed liquid}

One solution of the stationarity conditions $(3.76)$ is the point $\bar{\Omega}=\overline{\widetilde{\Omega}}=\bar{\Psi}=$ 0 corresponding to the homogeneous liquid. To assess its stability, we consider the Landau expansion to leading order about this point, concentrating on the incompressible limit:

$$
\begin{aligned}
\frac{2 n}{N} \mathcal{F}_{n}(\bar{\Psi}, \overline{\tilde{\Omega}}, \bar{\Omega}) & =\sum_{\alpha, \boldsymbol{k}}^{\prime} g_{\mathrm{D}}\left(\boldsymbol{k}^{2}\right)\left|\tilde{\Omega}_{\boldsymbol{k}}^{\alpha}\right|^{2}+\overline{\sum_{\hat{k}}}\left(\frac{1}{\mu}-g_{\mathrm{D}}\left(\hat{k}^{2}\right)\right)\left|\Omega_{\hat{k}}\right|^{2} \\
& +\sum_{\alpha, \boldsymbol{k}}^{\prime}\left(\frac{1}{\chi^{\alpha}}-\left(1-q^{2}\right) g_{\mathrm{D}}\left(\boldsymbol{k}^{2}\right)\right)\left|\Psi_{\boldsymbol{k}}^{\alpha}\right|^{2}+\mathcal{O}\left(\tilde{\Omega}^{2}, \Omega^{2}, \Psi^{2}\right),
\end{aligned}
$$


with the Debye function $g_{\mathrm{D}}\left(k^{2}\right)$ defined in appendix D.2.1. (For the Landau expansion retaining terms due to compressibility see section 3.5.7.) In anticipation of the replica limit $n \rightarrow 0$, we have taken $V^{-n} \rightarrow 1$. From the quadratic coefficients, we can read off the stability limits for the homogeneously mixed liquid against gelation and demixing before and after crosslinking: As the Debye function decreases monotonically with $k$ from one to zero, they are given by

$$
\mu<1, \quad \chi_{\mathrm{p}}<\frac{1}{1-q^{2}}, \quad \text { and } \quad \chi_{\mathrm{m}}<\frac{1}{1-q^{2}} .
$$

The incompressible limit guarantees the stability against mass density inhomogeneities $\left(\lambda_{\mathrm{p}}, \lambda_{\mathrm{m}}>\mu\right)$.

It should be noted that the conditions (3.78) for $\chi_{\mathrm{p}}, \chi_{\mathrm{m}}$ indeed denote the limits of stability. In mean-field theory, the phase separation transition for symmetric blends is of second order, so the phase transition point and the spinodal coincide. In asymmetric blends the transition is of first order, occurring already at lower $\chi$, and the spinodal just indicates the breakdown of stability of an undercooled mixture.

\subsubsection{Crosslinking in the homogeneously mixed state}

A liquid polymer blend cooled below the demixing threshold will separate macroscopically. Gelation in this state would result in just two pieces of gel, one rich in $A$ chains, the other rich in $B$ chains. It is much more interesting to consider a gel prepared from a homogeneous melt, as this still has a tendency to phase separate, yet into microphases rather than macroscopic ones. Moreover, it exhibits nontrivial charge correlations, which reflect both the situation at measurement and at the time of preparation. Therefore, the discussion will be restricted to systems prepared by crosslinking in a (possibly undercooled) homogeneously mixed blend, i.e. $\chi_{\mathrm{p}}<1 /\left(1-q^{2}\right)$.

Upon gelation, the saddle point value $\bar{\Omega}_{\hat{k}}=0$ will become unstable, so the Landau expansion of the effective free energy must be complemented by the nextto-leading-order term in $\Omega_{\hat{k}}$ and the term coupling $\Omega_{\hat{k}}$ and $\Psi_{\boldsymbol{k}}^{\alpha}$.

$$
\begin{aligned}
& \frac{2 n}{N} \mathcal{F}_{n}(\{\Psi, \Omega\})=\overline{\sum_{\hat{k}}}\left(\frac{1}{\mu}-g_{\mathrm{D}}\left(\hat{k}^{2}\right)\right)\left|\Omega_{\hat{k}}\right|^{2}+\sum_{\alpha, \boldsymbol{k}}^{\prime}\left(\frac{1}{\chi^{\alpha}}-\left(1-q^{2}\right) g_{\mathrm{D}}\left(\boldsymbol{k}^{2}\right)\right)\left|\Psi_{\boldsymbol{k}}^{\alpha}\right|^{2} \\
& -\sum_{\alpha_{1} \neq \alpha_{2}} \sum_{\boldsymbol{k}_{1,2}}^{\prime}\left(1-q^{2}\right) g_{\Psi^{2} \Omega}\left(\boldsymbol{k}_{1}^{2}, \boldsymbol{k}_{2}^{2}\right) \Psi_{\boldsymbol{k}_{1}}^{\alpha_{1}} \Psi_{\boldsymbol{k}_{2}}^{\alpha_{2}} \Omega_{\hat{k}} \cdot \delta_{\hat{k}+\boldsymbol{k}_{1} \otimes e_{\alpha_{1}}+\boldsymbol{k}_{2} \otimes e_{\alpha_{2}}, \hat{0}} \\
& -\frac{1}{3} \sum_{\hat{k}_{1,2,3}} g_{3}\left(\hat{k}_{1}, \hat{k}_{2}\right) \Omega_{\hat{k}_{1}} \Omega_{\hat{k}_{2}} \Omega_{\hat{k}_{3}} \cdot \delta_{\hat{k}_{1}+\hat{k}_{2}+\hat{k}_{3}, \hat{0}} \quad+\mathcal{O}\left(\Omega_{\hat{k}}^{4}, \Psi_{\boldsymbol{k}}^{4}\right)
\end{aligned}
$$

the vertex functions $g_{\Psi^{2} \Omega}\left(\boldsymbol{k}_{1}^{2}, \boldsymbol{k}_{2}^{2}\right)$ and $g_{3}\left(\hat{k}_{1}, \hat{k}_{2}\right)$ are defined in appendix D.2.1. On the mean-field level, gelation in the mixed state is not affected by the one-replica density field, the terms in $\tilde{\Omega}$ have therefore been omitted in eq. (3.79), see also section 3.5.7. 
To determine at which amount of crosslinking the gelation transition occurs, and how the order parameter $\bar{Q}$ looks like in the gel, we make a guess for the saddle point values of $\Omega$ and $\Psi$, which is verified subsequently. For the moment we assume that the external conditions remain unchanged after preparation, i.e. $\chi_{\mathrm{m}}=\chi_{\mathrm{p}}<1 /\left(1-q^{2}\right)$. Expecting that the introduction of crosslinks increase the stability of the mixed state, which is indeed shown in 3.5.4, we assume that the saddle point value of $\bar{\Psi}$ remains zero. Following $[76,77]$, we consider the order parameter hypothesis $\overline{\tilde{\Omega}}_{\boldsymbol{k}}^{\alpha}=0$,

$$
\bar{\Psi}_{\boldsymbol{k}}^{\alpha}=0 \quad \text { and } \quad \bar{\Omega}_{\hat{k}}=\delta_{\tilde{\boldsymbol{k}}, \mathbf{0}} \cdot f_{\text {gel }} \int_{0}^{\infty} \mathrm{d} \tau p(\tau) \exp \left(-\tilde{\boldsymbol{k}}^{2} / 2 \tau\right),
$$

the latter being the adaption of hypothesis (3.28) to the replica-field formulation. The gel fraction $f_{\text {gel }}$ of chains that are localised chains and the distribution $p(\tau)$ of localisation lengths have to be determined self-consistently according to the stationarity conditions (3.76). The shorthand $\tilde{\boldsymbol{k}}:=\sum_{\alpha=0}^{n} \boldsymbol{k}^{\alpha}$ denotes the sum of the $(n+1)$ spatial vectors in $\hat{k}$. The saddle point of $\Omega_{\hat{k}}$ is assumed to vanish except for $\tilde{\boldsymbol{k}}=\mathbf{0}$, cf. section 3.3 .1 and eq. (3.66).

The first two of the stationarity conditions (3.76) are satisfied identically for any $f_{\text {gel }}$ and $p(\tau)$. This also holds true when using the exact formulation (3.72) of the effective free energy, since $\left\langle\mathrm{e}^{-\mathrm{i} \boldsymbol{k} \boldsymbol{r}^{\alpha}(s)} \exp \left\{\bar{\sum}_{\hat{k}^{\prime}} \bar{\Omega}_{\hat{k}^{\prime}} \int_{0}^{1} d s^{\prime} \mathrm{e}^{\mathrm{i} \hat{k}^{\prime} \hat{r}\left(s^{\prime}\right)}\right\}\right\rangle_{n+1}^{W}=0$, because the Wiener average vanishes unless the involved wavevectors sum to zero within each replica.

On the saddle point (3.80), the third condition in (3.76) is independent of the charge density and of $\chi_{\mathrm{p}}$ and $\chi_{\mathrm{m}}$, and the task of determining $q$ and $p(\tau)$ on the saddle-point level is exactly the same as in the pure gelation problem in [76, 77]. Hence we may adopt the result therein, which in the present notation reads

$$
\bar{\Omega}_{\hat{k}}= \begin{cases}0, & \text { for } \mu \leq 1 \text { (liquid) } \\ \delta_{\tilde{\boldsymbol{k}}, 0} \cdot \mu(\mu-1) \cdot w\left(\hat{k}^{2} / 2(\mu-1)\right), & \text { for } \mu>1 \text { (gel) }\end{cases}
$$

with the scaling function $w(x)$ defined in appendix D.3, decaying from $w(0)=2$ to zero on the scale $x \sim 1$. For $\mu \geq 1$, the gel fraction is approximately given by $f_{\text {gel }} \approx 2(\mu-1)$.

\subsubsection{Stability of the mixed gel}

As long as the amount of crosslinks stays below the critical value for gelation, the crosslinks do not affect the stability versus phase separation, and the separation still occurs at $\chi_{\mathrm{m}}=1 /\left(1-q^{2}\right)$. In the following, we shall therefore concentrate on the case of gels prepared from a homogeneous melt, i.e. $\mu>1$ and $\chi_{\mathrm{p}}<1 /\left(1-q^{2}\right)$, and compute the stability limit of the mixed regime, which corresponds to the saddle point value $\Psi=0$. 


\section{Crosslinked homopolymer blends}

The stability of this saddle point is determined by the eigenvalue spectrum of the Hessian matrix of the effective free energy (3.72) with respect to $\Psi_{\boldsymbol{k}}^{\alpha}$, evaluated at the saddle point (3.80):

$$
\begin{aligned}
& \left.\frac{1}{N} \frac{\partial^{2} \mathcal{F}_{n}}{\partial \Psi_{\boldsymbol{k}_{1}}^{\alpha_{1}} \partial \Psi_{\boldsymbol{k}_{2}}^{\alpha_{2}}}\right|_{\{\bar{\Psi}, \bar{\Omega}\}}=\delta_{\boldsymbol{k}_{1}+\boldsymbol{k}_{2}, \mathbf{0}} \cdot \frac{\delta^{\alpha_{1}, \alpha_{2}}}{\chi^{\alpha_{1}}} \\
& -\delta_{\boldsymbol{k}_{1}+\boldsymbol{k}_{2}, \mathbf{0}} \cdot \frac{\left(1-q^{2}\right) \int_{0}^{1} \mathrm{~d} s_{1} \mathrm{~d} s_{2}\left\langle\mathrm{e}^{-\mathrm{i} \boldsymbol{k}_{1}\left(\boldsymbol{r}\left(s_{1}\right)-\boldsymbol{r}\left(s_{2}\right)\right)} \exp \left\{\mu \overline{\sum_{\hat{k}}} \bar{\Omega}_{\hat{k}} \int_{0}^{1} \mathrm{~d} s \mathrm{e}^{-\mathrm{i} \hat{k} \hat{r}(s)}\right\}\right\rangle_{n}^{\mathrm{W}}}{\left\langle\exp \left\{\mu \overline{\sum_{\hat{k}}} \bar{\Omega}_{\hat{k}} \int_{0}^{1} \mathrm{~d} s \mathrm{e}^{-\mathrm{i} \hat{k} \hat{r}(s)}\right\}\right\rangle_{n}^{\mathrm{W}}}
\end{aligned}
$$

where we have used that $\bar{\Omega}_{\hat{k}}=0$ for $\tilde{\boldsymbol{k}} \neq \mathbf{0}$ and that the Wiener averages vanish unless the wavevectors in each replica sum to zero. There is no coupling between different wavevectors, thus the Hessian matrices for different $\boldsymbol{k}$ can be considered independently. We restrict the discussion to a weak gel, i.e. $\mu-1 \ll 1$, so that the saddle point value of $\Omega_{\hat{k}}$ is small, and eq. (3.82) can be approximated by its expansion to first order in $\bar{\Omega}_{\hat{k}}$ :

$$
\left.\frac{\partial^{2} \mathcal{F}_{n}}{\partial \Psi_{\boldsymbol{k}}^{\alpha_{1}} \partial \Psi_{-\boldsymbol{k}}^{\alpha_{2}}}\right|_{\{\bar{\Psi}, \bar{\Omega}\}} \approx N\left(1-q^{2}\right) \cdot A_{\alpha_{1} \alpha_{2}}(k),
$$

with the approximate Hessian $\mathbf{A} \in \mathbb{R}^{(n+1) \times(n+1)}$ given by

$$
\mathbf{A}(k):=\left(\begin{array}{cccc}
c & -b & \cdots & -b \\
-b & a & \ddots & \vdots \\
\vdots & \ddots & \ddots & -b \\
-b & \ldots & -b & a
\end{array}\right),
$$

and depending only on the scalar wavenumber $k:=|\boldsymbol{k}| \geq 0$ due to isotropy. The entries of $\mathbf{A}(k)$ read

$$
\begin{aligned}
a & :=\left(\frac{1}{\left(1-q^{2}\right) \chi_{\mathrm{m}}}-g_{\mathrm{D}}\left(k^{2}\right)\right) \\
b & :=\mu(\mu-1) g_{\Psi^{2} \Omega}\left(k^{2}\right) w\left(k^{2} /(\mu-1)\right)
\end{aligned}
$$

with the explicit value (3.81) of the gelation order parameter, and

$$
c=\left(\frac{1}{\left(1-q^{2}\right) \chi_{\mathrm{p}}}-g_{\mathrm{D}}\left(k^{2}\right)\right) .
$$




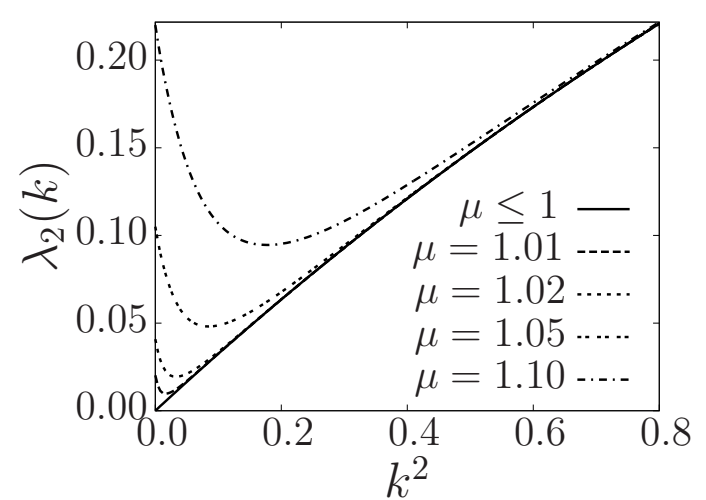

(a)

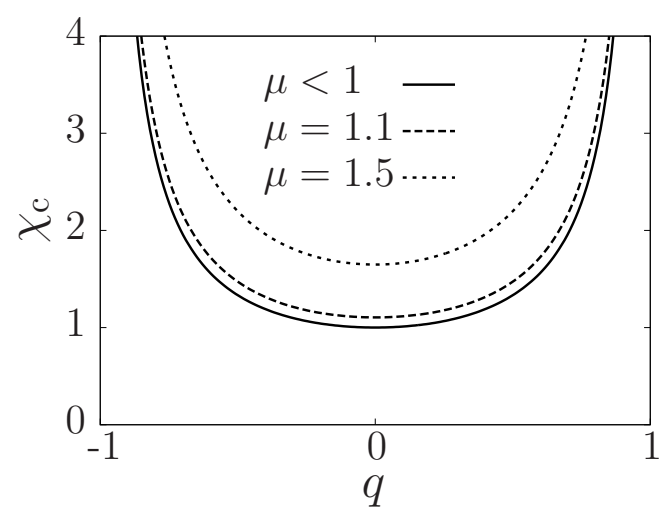

(b)

Figure 3.1.: Stability of the mixed gel. (a) Stability parameter $\lambda_{2}(k)$ for $\chi_{\mathrm{m}}=$ $1 /\left(1-q^{2}\right)$ as a function of $k^{2}$. (b) Spinodal $\chi_{\mathrm{c}}(\mu)$ as a function of the asymmetry $q$ for the liquid $(\mu<1)$ and gels with different strengths $(\mu=1.1$ and $\mu=1.5)$.

(The approximate Hessian $(3.84-3.87)$ can alternatively be derived from the expansion (3.79) by replacing $\Omega_{\hat{k}}$ with $\bar{\Omega}_{\hat{k}}$.)

The stability of the homogeneous state is equivalent to the positivity of the matrix $\mathbf{A}$. In the limit $n \rightarrow 0$, the eigenvalues are given by

$$
\lambda_{1}(k):=c \quad \text { and } \quad \lambda_{2}(k):=a+b,
$$

which are non and $n$-fold degenerate, respectively; see eq. (D.87) in appendix D.4.1.

Because the gel is prepared from a mixed liquid, the first eigenvalue, which indicates the stability of the melt before crosslinking, is always positive, so the stability condition reduces to $\lambda_{2}>0$, or equivalently, $\chi_{\mathrm{m}}<\chi_{\mathrm{c}}(\mu)$ with

$$
\left(1-q^{2}\right) \chi_{\mathrm{c}}(\mu):=1 / \max _{k}\left\{g_{\mathrm{D}}\left(k^{2}\right)-\mu(\mu-1) g_{\Psi^{2} \Omega}\left(k^{2}\right) w\left(k^{2} /(\mu-1)\right)\right\} .
$$

Figure 3.1(a) shows $\lambda_{2}(k)$ for $\chi_{\mathrm{m}}=1 /\left(1-q^{2}\right)$. With increasing $\chi_{\mathrm{m}}$, the whole curve is shifted to smaller values, and the minimum, located at $k_{\mathrm{c}}>0$, eventually becomes negative, indicating a finite wavelength instability against phase separation. The corresponding wavenumber reads

$$
k_{\mathrm{c}}:=\operatorname{argmax}_{k>0}\left\{g_{\mathrm{D}}\left(k^{2}\right)-\mu(\mu-1) g_{\Psi^{2} \Omega}\left(k^{2}\right) w\left(k^{2} /(\mu-1)\right)\right\} .
$$

We consider a weak gel, thus $\mu(\mu-1) \approx \mu-1$. Furthermore, $w\left(k^{2} /(\mu-1)\right)$ decays fast with $k$, so that the Debye function can be approximated by the leadingorder expansion $g_{\mathrm{D}}\left(k^{2}\right) \approx 1-k^{2} / 3$, and $g_{\Psi^{2} \Omega} \approx 1$ even by its $k \rightarrow 0$ limit. In this approximation, $k_{\mathrm{c}}$ results from

$$
0=\left.\frac{\partial \lambda_{2}}{\partial k^{2}}\right|_{k=k_{\mathrm{c}}} \approx \frac{1}{3}+w^{\prime}\left(k_{\mathrm{c}}^{2} /(\mu-1)\right),
$$


which leads to $k_{\mathrm{c}}^{2} \approx 1.61(\mu-1)$ and $\left(1-q^{2}\right) \chi_{\mathrm{c}}-1 \approx 0.98(\mu-1)$. The numerical analysis without these approximations yields

$$
k_{\mathrm{c}}^{2}=1.61 \cdot(\mu-1)+1.75 \cdot(\mu-1)^{2}+\mathcal{O}\left((\mu-1)^{3}\right)
$$

and

$$
\left(1-q^{2}\right) \chi_{\mathrm{c}}-1=0.98 \cdot(\mu-1)+0.70 \cdot(\mu-1)^{2}+\mathcal{O}\left((\mu-1)^{3}\right) .
$$

To summarise, the gel network stabilises the mixed state, shifting the phase separation threshold $\chi_{\mathrm{c}}$ to higher values corresponding to lower temperatures. Likewise, an asymmetric composition leads to a higher stability limit. Both effects can be seen in fig. 3.1(b), which shows the spinodal $\chi_{\mathrm{c}}$ as a function of the asymmetry $q$ for the liquid and two gels generated with different amounts of crosslinking. Moreover, the demixing instability first occurs for nonzero wavenumbers, indicating that the macroscopic phase separation is replaced by microphase separation in the gel state. This issue will be addressed in section 3.5.7.

\subsubsection{Phase diagram}

This section deals with the phase diagram of crosslinked blends in the symmetric case $N_{A}=N_{B}$, for which the spinodals and the phase separation lines coincide, hence we let $q=0$. (The spinodals of asymmetric blends can, however, be recovered by replacing $\chi$ with $\left.\left(1-q^{2}\right) \chi\right)$.

The state of the gel depends on the history of gelation, which, in our model, is fully characterised by two parameters: The incompatibility $\chi_{\mathrm{p}}$ at the time of preparation, and the strength $\mu$ of crosslinking. However, these parameters are fixed upon preparation, so that the phase diagram of a particular sample is one dimensional, just depending on the incompatibility (or inverse temperature) $\chi_{\mathrm{m}}$ at the time of measurement.

Nevertheless, to achieve an overview it would be useful to draw a three-dimensional diagram with additional axes for $\chi_{\mathrm{p}}$ and $\mu$ as fixed parameters, so to speak "stacking" the one-dimensional phase diagrams of all possible gels. In gels prepared from a homogeneous melt, i.e. $\chi_{\mathrm{p}}<1$, the gelation and phase separation thresholds are independent of $\chi_{\mathrm{p}}$, see eqs.(3.81), (3.89) and (3.90). Hence, the cross sections of the diagram for different $\chi_{\mathrm{p}}<1$ are identical, so it is not necessary to draw the $\chi_{\mathrm{p}}$ axis. It should be noted that, in contrast to the transition lines, the charge density fluctuations do indeed depend on the temperature at preparation; they will be discussed in section 3.5.6.

The phase diagram in the $\chi_{\mathrm{m}}-\mu$-plane, with $\chi_{\mathrm{p}}$ as a fixed parameter, is shown in fig. 3.2. The dashed line $\mu=1$ separates the gel state and the liquid state. The latter is further divided into a mixed and a macroscopically phase separated liquid at $\chi_{\mathrm{p}}=1$ (solid line). The dotted line $\chi_{\mathrm{m}}=\chi_{\mathrm{c}}$ indicates the transition from the homogeneous to a microphase separated gel. 


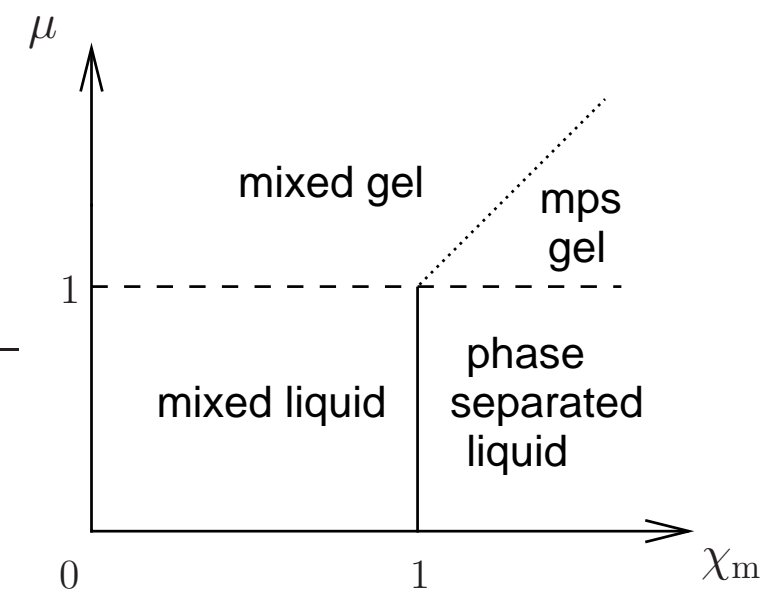

Figure 3.2: $\quad$ Phase diagram of the symmetric polymer blend (schematic) in the $\chi_{\mathrm{m}}-\mu$-plane, crosslinked in the homogeneous state $\left(\chi_{\mathrm{p}}<1\right)$.

\subsubsection{Charge density correlations in the mixed gel}

To obtain the charge density correlations defined in section 3.3 .2 we study the second moments of the auxiliary fields $\Psi_{\boldsymbol{k}}^{\alpha}$. The latter are calculated on the Gaussian level, i.e. with the second-order expansion of the effective free energy,

$$
\left\langle\Psi_{\boldsymbol{k}}^{\alpha_{1}} \Psi_{-\boldsymbol{k}}^{\alpha_{2}}\right\rangle_{n+1}^{\mathcal{F}} \approx \frac{\int \mathrm{d}^{n+1} \Psi \Psi^{\alpha_{1}}\left(\Psi^{\alpha_{2}}\right)^{*} \exp \left\{-\frac{\gamma N}{2} \boldsymbol{\Psi}^{\dagger} \mathbf{A}(k) \boldsymbol{\Psi}\right\}}{\int \mathrm{d}^{n+1} \Psi \exp \left\{-\frac{\gamma N}{2} \boldsymbol{\Psi}^{\dagger} \mathbf{A} \boldsymbol{\Psi}\right\}}=\frac{\mathbf{A}_{\alpha_{1} \alpha_{2}}^{-1}}{\gamma N},
$$

where $\gamma$ denotes the asymmetry parameter

$$
\gamma:=1-q^{2}
$$

The elements $\mathbf{A}_{\alpha_{1} \alpha_{2}}^{-1}$ of the inverse of the Hessian matrix (3.84) are computed in appendix D.4.2. With these elements and with eqs. (3.68) and (3.69) we obtain

$$
S(k) \approx \frac{1}{\chi_{\mathrm{m}}^{2}}\left(\left.\frac{1}{\gamma} \lim _{n \rightarrow 0} \mathbf{A}_{\alpha \alpha}^{-1}\right|_{\alpha>0}-\chi_{\mathrm{m}}\right)=\frac{\gamma}{\left(\gamma \chi_{\mathrm{m}}\right)^{2}}\left(\frac{b\left(\lambda_{1}+b\right)}{\lambda_{1} \cdot \lambda_{2}^{2}}+\frac{1}{\lambda_{2}}-\gamma \chi_{\mathrm{m}}\right)
$$

and

$$
\left.S_{\mathrm{gl}}(k) \approx \frac{1}{\chi_{\mathrm{m}}^{2}} \cdot \frac{1}{\gamma} \lim _{n \rightarrow 0} \mathbf{A}_{\alpha_{1} \alpha_{2}}^{-1}\right|_{\substack{\alpha_{1} \neq \alpha_{2} \\ \alpha_{1,2}>0}}=\frac{\gamma}{\left(\gamma \chi_{\mathrm{m}}\right)^{2}} \cdot \frac{b\left(\lambda_{1}+b\right)}{\lambda_{1} \cdot \lambda_{2}^{2}} .
$$

(The eigenvalues and the off-diagonal entry $b$ of $\mathbf{A}$ are defined in eqs. (3.86) and (3.88).) The variance or connected correlation is given by

$$
S_{\mathrm{th}}(k)=S(k)-S_{\mathrm{gl}}(k)=\frac{\gamma}{\left(\gamma \chi_{\mathrm{m}}\right)^{2}}\left(\frac{1}{\lambda_{2}}-\gamma \chi_{\mathrm{m}}\right) .
$$

\section{Restriction to symmetric blends}

In the following discussion of $S(k), S_{\mathrm{gl}}(k)$ and $S_{\mathrm{th}}(k)$ and the inherent length scales, we shall confine ourselves to the case of symmetric blends, yet without loss 


\section{Crosslinked homopolymer blends}

of generality: The scattering functions of the asymmetric case are recovered via multiplication by $\gamma=\left(1-q^{2}\right)$ and the rescalings $\chi_{\mathrm{p}} \rightarrow \gamma \chi_{\mathrm{p}}$ and $\chi_{\mathrm{m}} \rightarrow \gamma \chi_{\mathrm{m}}$. Furthermore, the distance to phase separation is replaced with the distance to the spinodal in the asymmetric case. In the range between the equilibrium phase transition and the spinodal, the results then describe the undercooled mixture.

\section{Length-scales}

The correlation functions are characterised by three length-scales which are determined by the parameters $\left(\mu, \chi_{\mathrm{p}}\right)$ of preparation and the conditions $\chi_{\mathrm{m}}$ at measurement (as pointed out above, we concentrate on the symmetric case, $q=0$ ):

First, there is the typical localisation length of the monomers in the gel fraction, i.e. the mean mesh size of the gel. From eq. (3.81) we can infer that this length-scale is roughly given by $2 \pi \xi_{1}$ with

$$
\xi_{1}:=1 / \sqrt{\mu-1} .
$$

Second, there is the decay length of the pre-critical demixing fluctuations with $k$ prior to gelation. This approximately reads $\xi_{\mathrm{p}} / 2 \pi$ with

$$
\xi_{\mathrm{p}}:=1 / \sqrt{1-\chi_{\mathrm{p}}}
$$

Fluctuations towards macroscopic demixing can also occur in the gel, at the time of measurement, provided the network is weak enough to permit large-scale displacements (see the following part for details). The third length scale is the correlation length $\xi_{\mathrm{m}} / 2 \pi$ of these fluctuations, given by

$$
\xi_{\mathrm{m}}:=1 / \sqrt{\chi_{\mathrm{c}}(\mu)-\chi_{\mathrm{m}}} \approx 1 / \sqrt{1-\chi_{\mathrm{m}}} .
$$

The last approximation is only valid for $\mu-1 \ll 1$ and $\chi_{\mathrm{m}}$ not too close to $\chi_{\mathrm{c}}$, i.e. for small $\xi_{1}$ and $\xi_{\mathrm{m}}$.

The three length-scales measure, or are given by, the inverse distance to the phase transitions of gelation and demixing in the pre-crosslinking blend, and microphase separation in the gel; hence they grow large when approaching their respective transitions. Ignoring the factor of $2 \pi$, we shall refer to $\xi_{1}, \xi_{\mathrm{p}}$ and $\xi_{\mathrm{m}}$ as "the localisation length" or "mesh size" and "the correlation lengths" of the preparation and measurement ensemble, respectively.

In the following we shall essentially discuss three limiting regimes, in which the correlation functions are determined by one of the three length-scales. These regimes are illustrated in fig. 3.3 (a modified version of fig. 3.2). The plot includes the paths, or histories, of three samples. The vertical part of the path (grey) indicates the preparation conditions $\mu$ and $\chi_{\mathrm{p}}$ by its height and horizontal position; the incompatibility $\chi_{\mathrm{m}}$ at the time of measurement is indicated by the end point (black). The phase separation line refers to $\chi_{\mathrm{m}}$ only. The three paths describe (A) a gel prepared in a liquid very close to demixing, i.e. with $\xi_{\mathrm{p}} \gg \xi_{1}, \xi_{\mathrm{m}}$; (B) a very weak gel, i.e. with $\xi_{1} \gg \xi_{\mathrm{p}}, \xi_{\mathrm{m}}$, and (C) a gel measured very close to microphase transition, i.e. with $\xi_{\mathrm{m}} \gg \xi_{1}, \xi_{\mathrm{p}}$. 


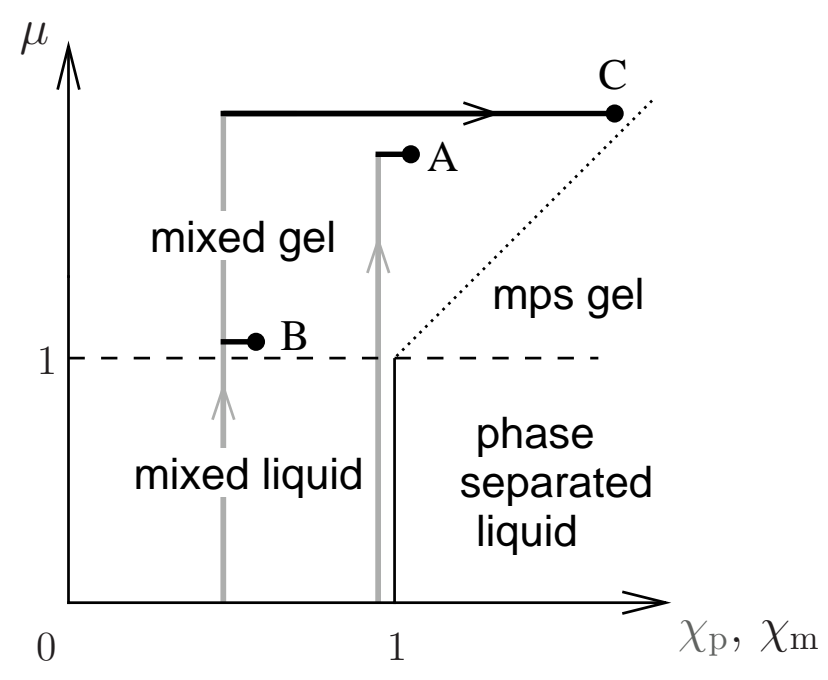

Figure 3.3: Histories of different samples visualised by different paths. The vertical parts (grey) and the dots (black) indicate the preparation parameters $\left(\mu, \chi_{\mathrm{p}}\right)$ and the conditions $\chi_{\mathrm{m}}$ at measurement, respectively. The three exemplary paths show a gel prepared close to demixing (A), a weakly crosslinked gel (B) and a gel measured close to microphase separation $(\mathrm{C})$.

\section{Glassy correlations}

The glassy correlation function $S_{\mathrm{gl}}(k)$, which describes the persistent charge inhomogeneities, consists of two parts: the "frozen-in" fluctuations, which originate from the thermal fluctuations present at the instant of crosslinking that are partially preserved by the network, and the precursors of microphase separation, which are seeded by the frozen-in fluctuations and the disorder due to the randomness of crosslinking; these precursors grow large on approaching the microphase transition.

Sufficiently far away from the transition, i.e. for small $\xi_{\mathrm{m}}, S_{\mathrm{gl}}$ is dominated by the frozen pre-crosslinking fluctuations, with a high weight at zero wavenumber and decaying with $k$. Prior to preparation, the fluctuations decay on the scale $k \xi_{\mathrm{p}} \sim 1$, yet the gel network can only preserve structures larger than or comparable to its localisation length. Hence the larger of the two lengths $\xi_{\mathrm{p}}$ and $\xi_{1}$ determines the scale of the frozen fluctuations:

In a gel prepared close to phase separation, i.e. $\xi_{\mathrm{p}} \gg\left(\xi_{1}, \xi_{\mathrm{m}}\right) \gg 1$, corresponding to path "A" in fig. 3.3, the pre-crosslinking fluctuations are large-scaled enough to be frozen-in in the network. In this case, eq. (3.96) can be approximated for small $k \ll \xi_{\mathrm{p}}^{-1}$ by

$$
S_{\mathrm{gl}}(k) \approx \frac{1}{\left(c_{1}^{2}+\frac{1}{2} \xi_{1}^{2} / \xi_{\mathrm{m}}^{2}\right)^{2}} \cdot \frac{\xi_{\mathrm{p}}^{2}}{1+k^{2} \xi_{\mathrm{p}}^{2} / 3}
$$

with a constant $c_{1}^{2}:=1-\left.\frac{1}{2} \xi_{1}^{2}\left(\chi_{\mathrm{c}}-1\right)\right|_{\xi_{1} \rightarrow \infty} \approx 0.5$, see eq. (3.89). Thus, $S_{\mathrm{gl}}(k)$ is proportional to $\xi_{\mathrm{p}}^{2}$ and decays on the scale $k \sim \xi_{\mathrm{p}}^{-1}$ of the fluctuations of the preparation ensemble. An example is shown in fig. 3.4(a).

In a weak gel, crosslinked just beyond the gelation threshold, i.e. $\xi_{1} \gg\left(\xi_{\mathrm{p}}, \xi_{\mathrm{m}}\right) \gg$ 1 (path "B"), the mesh size of the network is large, and the frozen correlations are cut off at the inverse localisation length $\xi_{1}^{-1}$. For small $k$, the glassy correlations are approximately given by

$$
S_{\mathrm{gl}}(k) \approx \xi_{\mathrm{m}}^{4} / \xi_{\mathrm{l}}^{2} \cdot w\left(k^{2} \xi_{1}^{2}\right),
$$




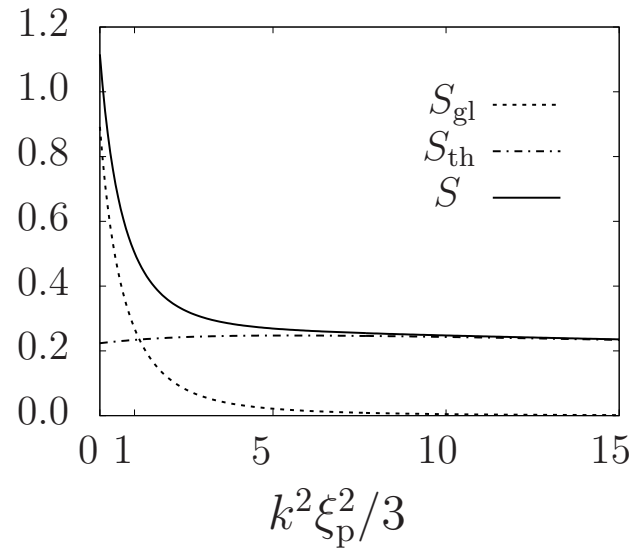

(a)

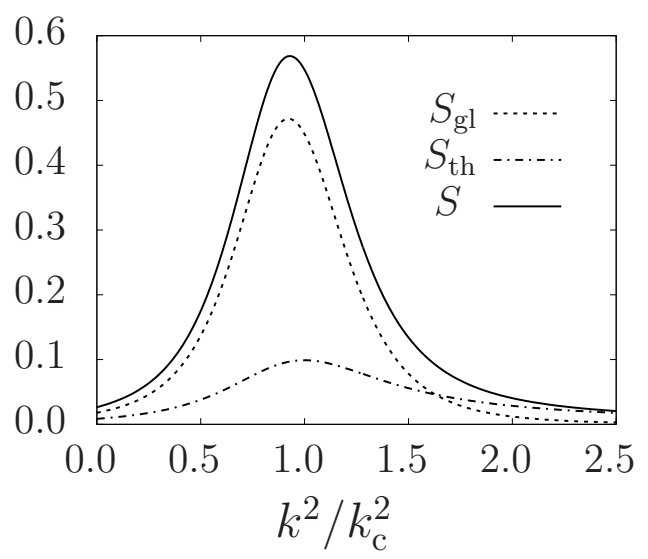

(c)

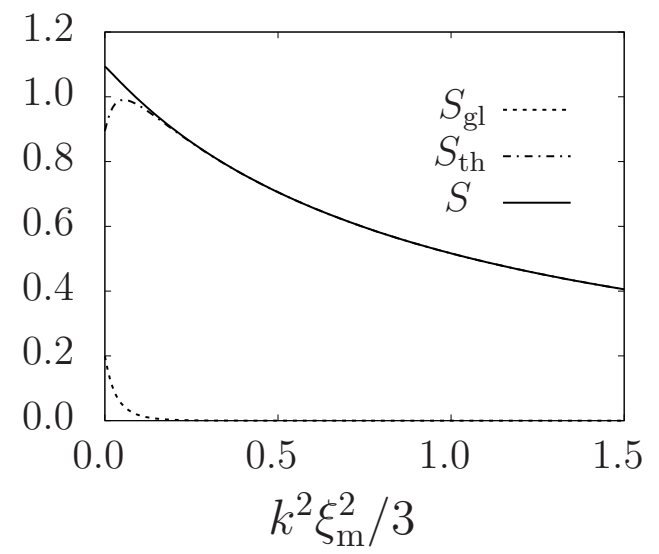

(b)

Figure 3.4.: Correlation functions in the limiting scenarios: (a) Gel prepared close to phase separation: $S_{\mathrm{gl}}, S_{\mathrm{th}}$ and $S$ in units of $4 \xi_{\mathrm{p}}^{2} \xi_{\mathrm{m}}^{4} / \xi_{\mathrm{l}}^{4}$ for $\xi_{\mathrm{l}}^{2}=10^{2}, \xi_{\mathrm{p}}^{2}=10^{3}$ and $\xi_{\mathrm{m}}^{2}=10$. (b) Weak gel: $S_{\mathrm{gl}}, S_{\mathrm{th}}$ and $S$ in units of $\xi_{\mathrm{m}}^{2}$ for $\xi_{1}^{2}=10^{2}, \xi_{\mathrm{p}}^{2}=10$ and $\xi_{\mathrm{m}}^{2}=10$. (c) Measurement close to microphase separation: $S_{\mathrm{gl}}, S_{\mathrm{th}}$ and $S$ in units of $\xi_{\mathrm{m}}^{4} / \xi_{1}^{2}$ for $\xi_{1}^{2}=10^{2}, \xi_{\mathrm{p}}^{2}=10$ and $\xi_{\mathrm{m}}^{2}=10^{3}$. The wavenumber squares are measured in units of $k_{\mathrm{c}}^{2}$.

hence they decay on the scale $k \sim \xi_{1}^{-1}$, yet grow when approaching microphase separation. An example is given in fig. 3.4(b).

The cross-over between the two cases discussed so far is demonstrated in fig. 3.5, showing $S_{\mathrm{gl}}(k) / S_{\mathrm{gl}}(0)$ far from the microphase transition for $\xi_{1}=10$. For the leftmost curve, the correlation length of the pre-crosslinking fluctuations is much larger than the localisation length of the gel, $\xi_{\mathrm{p}}^{2}=10^{5} \gg \xi_{1}^{2}=100$, so that the fluctuations are accurately frozen-in, and $S_{\mathrm{gl}}$ decays on the scale $k \sim \xi_{\mathrm{p}}^{-1}$. Upon decreasing $\xi_{\mathrm{p}}$, the curves are shifted to higher $k$ until the correlation length becomes comparable to the localisation length, which eventually cuts off the preservation of the fluctuations at $k \sim \xi_{1}^{-1}$. The inset shows the half width at half maximum $k_{1 / 2}$ of the curves in the main plot; it increases with decreasing $\xi_{\mathrm{p}}$ until it saturates at 


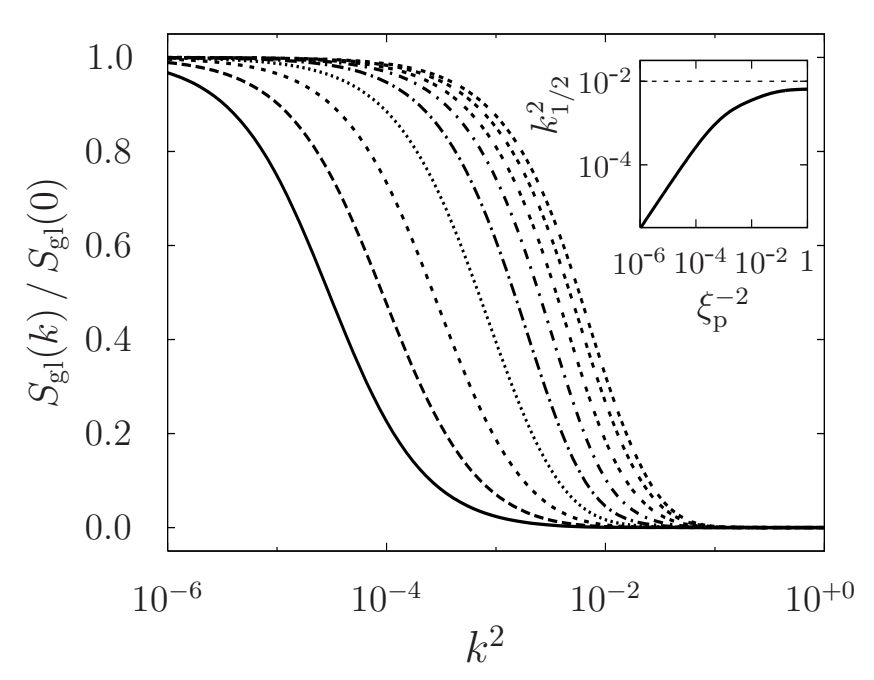

Figure 3.5: Scale crossover in the glassy correlation function $S_{\mathrm{gl}}$, normalised to the value at $k=0$ : crossover from preparation close to demixing to a weak gel, with $\chi_{\mathrm{m}}=0.1$, $\xi_{1}^{2}=10^{2}$, and $\xi_{\mathrm{p}}^{2}=$ $10^{5}, 10^{4.5}, \ldots, 10^{1}$ (from left to right). Inset: Halfwidth $k_{1 / 2}^{2}$ of $S_{\mathrm{gl}}(k)$, crossing over from $\xi_{\mathrm{p}}^{-2}$ to $\xi_{\mathrm{l}}^{-2}$ (dashed line).

about $\xi_{1}=10^{-2}$ (dashed line).

Close to microphase separation, i.e. $\xi_{\mathrm{m}} \gg\left(\xi_{1}, \xi_{\mathrm{p}}\right) \gg 1$, corresponding to path "C" in fig. 3.3, $S_{\mathrm{gl}}(k)$ is dominated by the critical fluctuations towards microphase separation, seeded by the frozen-in randomness in the gel. In the absence of crosslinks, there would be large-scale fluctuations towards macroscopic demixing. In the gel, displacements are bounded from above roughly by the localisation length, so that $S_{\mathrm{gl}}(k)$ develops a peak at about $k_{\mathrm{c}} \approx 1.6 \xi_{\mathrm{l}}^{-1}$, where $\lambda_{2}$ becomes small (see eq. (3.92)); an example is shown in fig. 3.4(c). On approaching the microphase transition, the peak diverges as $\lambda_{2}^{-2}(k)$, and the glassy correlations approximately follow

$$
S_{\mathrm{gl}}(k) \propto b \lambda_{2}^{-2} \approx \frac{\xi_{1}^{-2} w\left(k^{2} \xi_{\mathrm{l}}^{2}\right)}{\left(\xi_{\mathrm{m}}^{-2}+c_{2} \cdot\left(k^{2}-k_{\mathrm{c}}^{2}\right)^{2}\right)^{2}}
$$

with a constant $c_{2}=w^{\prime \prime}\left(k_{\mathrm{c}}^{2} \xi_{\mathrm{l}}^{2}\right) /\left(2 k_{\mathrm{c}}^{2}\right)$.

\section{Thermal fluctuations}

Whereas $S_{\mathrm{gl}}(k)$ describes the time-persistent part of the correlations, so to speak their mean value, the function $S_{\mathrm{th}}(k)$ measures their variance due to thermal fluctuations. Interestingly, it is independent of the conditions at the time of crosslinking, so there are only two competing length-scales, $\xi_{1}$ and $\xi_{\mathrm{m}}$, and two limiting situations: The weak gel and the gel measured close to microphase separation.

In a weak gel, where $\xi_{1} \gg \xi_{\mathrm{m}} \gg 1$, corresponding to path "B" in fig. 3.3, the thermal fluctuations are hardly restricted by the network. They are only suppressed on scales larger the localisation length $\xi_{1}$, i.e. for $k \ll \xi_{1}^{-1}$. Apart from a dip at $k=0$, $S_{\mathrm{th}}(k)$ therefore looks like critical fluctuations towards macroscopic demixing, see fig. 3.4(b). Provided $\xi_{\mathrm{m}}$ is not too small, $S_{\mathrm{th}}(k)$ reduces to

$$
S_{\mathrm{th}}(k) \approx \frac{\xi_{\mathrm{m}}^{2}}{1+k^{2} \xi_{\mathrm{m}}^{2} / 3},
$$


for small $k$, decaying with a half width of $k_{1 / 2} \approx \sqrt{3} / \xi_{\mathrm{m}}^{2}$.

Close to microphase separation, i.e. $\xi_{\mathrm{m}} \gg \xi_{1} \gg 1$ (path "C" in fig. 3.3), the strong critical fluctuations are strongly suppressed by the rigidity of the network on scales larger than the localisation length. In this regime,

$$
S_{\mathrm{th}}(k) \propto \lambda_{2}^{-1} \approx \frac{1}{\xi_{\mathrm{m}}^{-2}+c_{2} \cdot\left(k^{2}-k_{\mathrm{c}}^{2}\right)^{2}}
$$

with the constant $c_{2}$ defined near eq. (3.104), hence $S_{\text {th }}(k)$ develops a peak at about $k_{\mathrm{c}} \sim \xi_{1}^{-1}$, as shown in fig. 3.4(c). On approaching the microphase transition, the peak diverges as $\xi_{\mathrm{m}}^{2}$.

\section{Scattering structure factor}

The behaviour of the structure factor $S=S_{\mathrm{gl}}+S_{\mathrm{th}}$ in the three limiting regimes can be inferred from the behaviour of $S_{\mathrm{gl}}$ and $S_{\mathrm{th}}$. A weak gel (path "B" in fig. 3.3) preserves only a small amount of the pre-crosslinking fluctuations and hardly hinders thermal fluctuations, so that $S(k) \approx S_{\mathrm{th}}(k)$ in this case, which decays on the scale $k \sim \xi_{\mathrm{m}}$, see fig. $3.4(\mathrm{~b})$.

In the other two cases, thermal fluctuations are suppressed by the rigidity of the network, and $S(k) \approx S_{\mathrm{gl}}(k)$. In a gel prepared close to phase separation (path "A" in fig. 3.3), the structure factor decays on the scale $k \sim \xi_{\mathrm{p}}$, see fig. 3.4(a)), whereas a gel measured close to microphase separation (path "C" in fig. 3.3) reveals a peak at $k \sim k_{\mathrm{c}}$, diverging at the transition, see fig. 3.4(c).

The crossovers between the three regimes are illustrated in fig. 3.6. Panel (a) shows the crossover between a gel prepared close to demixing and the situation close to microphase separation, driven by increasing $\xi_{\mathrm{m}}$ at constant $\xi_{\mathrm{p}} \gg \xi_{1}$ for a comparably strong gel. Initially, $S(k)$ is dominated by the well-preserved precrosslinking fluctuations with large weight at small $k$. On growing $\xi_{\mathrm{m}}, S(k)$ develops a peak at $k \sim k_{\mathrm{c}}$, which diverges as the microphase separation is approached. The opposite case is plotted in panel (b), where the closeness $\xi_{\mathrm{p}}$ to phase separation prior to crosslinking is increased at fixed $\xi_{\mathrm{m}} \gg \xi_{1}$ for a comparably high gel strength. Whereas the peak at $k \sim k_{\mathrm{c}}$ remains roughly constant, we observe the build-up of intensity at low $k$ due to the frozen-in fluctuations. Panel (c) shows the crossover from a weak gel to a gel prepared close to demixing. In the weak gel, the frozen-in fluctuations are cut off on the scale $k_{1 / 2}^{2} \sim 3 / \xi_{\mathrm{m}}^{2}$ (vertical line). As $\xi_{\mathrm{p}}$ increases, the pre-crosslinking fluctuations become stronger and more long-ranged, hence the weight at low $k$ grows, and the half width $k_{1 / 2}^{2}$ of $S(k)$ eventually decreases as $1 / \xi_{\mathrm{p}}^{2}$. The crossover from a weak gel to a gel near the microphase transition can finally be seen in panel $(\mathrm{d})$. For small $\xi_{\mathrm{m}}$, the structure factor is dominated by the partially frozen-in fluctuations, cut off at about $k \lesssim \xi_{1}$. The demixing fluctuations increase with $\xi_{\mathrm{m}}$, but are damped on length-scales larger than $\xi_{1}$ by the rigidity of the network. This effectively leads to a peak at $k \sim k_{\mathrm{c}}$, a precursor of microphase separation. An overview over the basic features of $S_{\mathrm{gl}}$, $S_{\text {th }}$, and $S$ in the three regimes is given in tab. 3.5.6. 


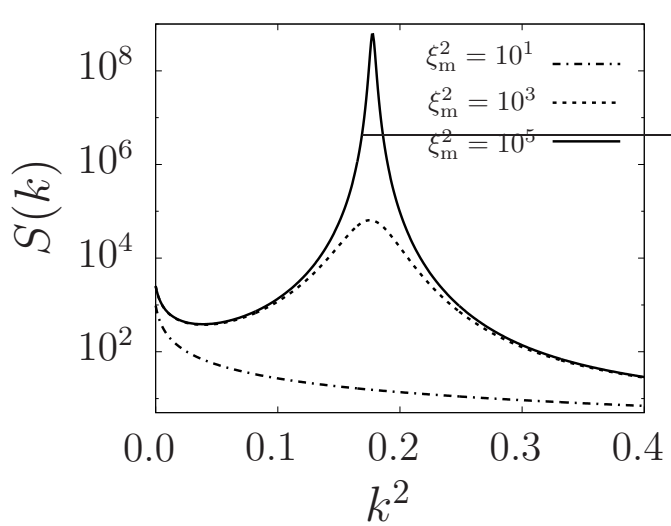

(a)

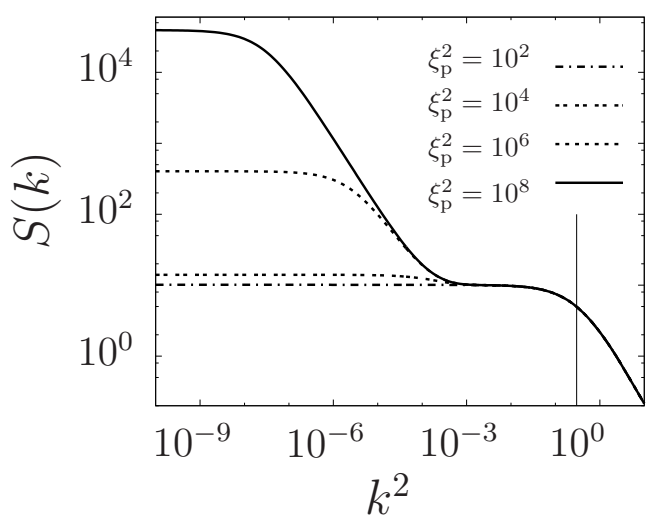

(c)

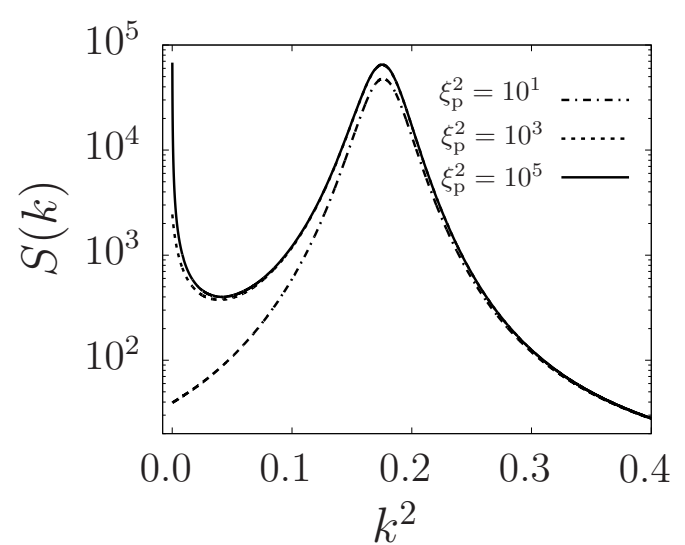

(b)

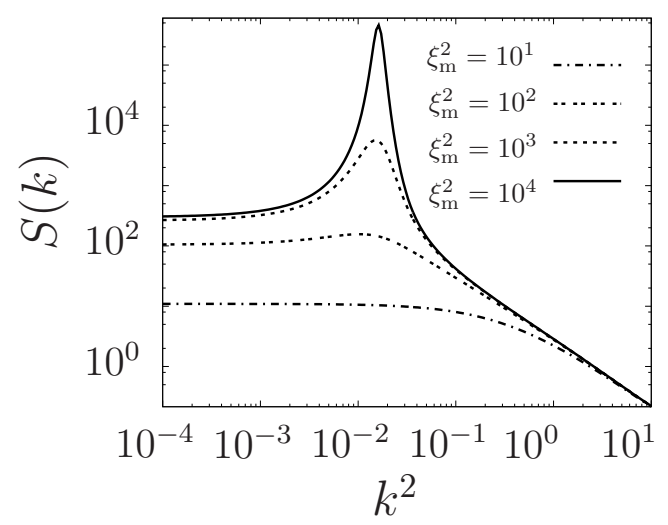

(d)

Figure 3.6.: Scale crossover in the structure factor $S(k)$. (a) From preparation near demixing to approaching microphase separation. . : $\xi_{\mathrm{m}}^{2}=10^{1}, 10^{3}, 10^{5}$ for $\xi_{\mathrm{p}}^{2}=10^{3}$ and $\xi_{\mathrm{l}}^{2}=10^{1}(\mathrm{~b}) \ldots$ and vice versa: $\xi_{\mathrm{p}}^{2}=10^{1}, 10^{3}, 10^{5}$ for $\xi_{\mathrm{m}}^{2}=10^{3}$ and $\xi_{1}^{2}=10^{1}$. (c) From a weak gel to preparation near demixing: $\xi_{\mathrm{p}}^{2}=10^{2}, 10^{4}, 10^{6}, 10^{8}$ for $\xi_{1}^{2}=10^{3}$ and $\xi_{\mathrm{m}}^{2}=10^{1}$. Vertical line: $k^{2}=3 \xi_{\mathrm{m}}^{-2}$. (d) From a weak gel to approaching mps: $\xi_{\mathrm{m}}^{2}=10^{1}, 10^{2}, 10^{3}, 10^{4}$ for $\xi_{1}^{2}=10^{2}$ and $\xi_{\mathrm{p}}^{2}=10^{1}$.

\section{Liquid and gel phase}

In the decay of the scattering functions in a gel prepared close to demixing, examined in the previous paragraphs, we have already seen the freezing-in of disorder in the gel. Nevertheless, it is interesting to complement this observation by comparing the correlations of the blend in the liquid and the gel phase, measured at the preparation conditions $\chi_{\mathrm{p}}=\chi_{\mathrm{m}}=: \chi$. They are given by

$$
S^{(1)}=\chi^{-2}\left(\frac{1}{\chi^{-1}-g_{\mathrm{D}}(k)}-\chi\right), \quad \quad S_{\mathrm{gl}}^{(1)}=0,
$$




$$
\text { (A) } \xi_{\mathrm{p}} \gg\left(\xi_{1}, \xi_{\mathrm{m}}\right)
$$

preparation near demixing
(B) $\xi_{1} \gg\left(\xi_{\mathrm{p}}, \xi_{\mathrm{m}}\right)$

weak gel
(C) $\xi_{\mathrm{m}} \gg\left(\xi_{1}, \xi_{\mathrm{p}}\right)$

measurement close to microphase separation

$$
\begin{aligned}
& \begin{aligned}
S_{\mathrm{gl}}(k) & \propto\left\{\begin{array}{lll}
\xi_{\mathrm{p}}^{2} \xi_{\mathrm{m}}^{4} / \xi_{1}^{4} & \text { for } \xi_{1} \gg \xi_{\mathrm{m}} & \\
\xi_{\mathrm{p}}^{2} & \text { for } \xi_{1} \ll \xi_{\mathrm{m}}
\end{array}\right. & \propto \xi_{\mathrm{m}}^{4} / \xi_{1}^{2} & \text { divergence } \propto \xi_{\mathrm{m}}^{4} / \xi_{1}^{2}
\end{aligned} \\
& S_{\mathrm{th}}(k) \quad \begin{array}{cr}
\propto \xi_{\mathrm{m}}^{2} & \text { divergence } \propto \xi_{\mathrm{m}}^{2} \\
\text { decay on scale } k \sim \xi_{\mathrm{m}}^{-1} & \text { peak at } k \sim k_{\mathrm{c}} \propto \xi_{\mathrm{l}}^{-2}
\end{array}
\end{aligned}
$$

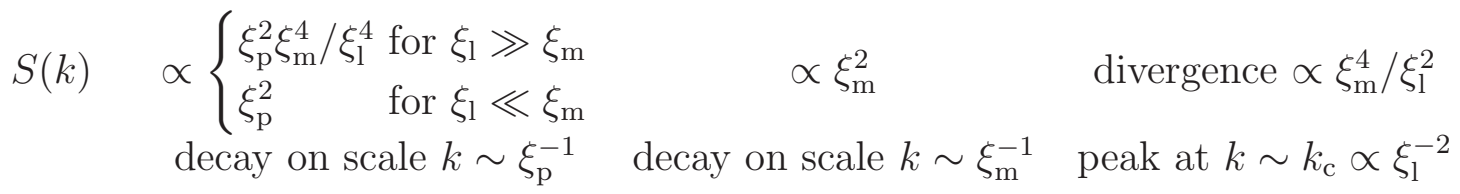

Table 3.1.: Behaviour of $S_{\mathrm{gl}}(k), S_{\mathrm{th}}(k)$, and $S(k)=S_{\mathrm{gl}}(k)+S_{\mathrm{th}}(k)$ in the limiting regimes corresponding to paths "A", "B" and "C" in fig. 3.3. Note that $S_{\text {th }}(k)$ is independent of $\xi_{\mathrm{p}}$.

$$
S^{(\mathrm{g})}=\chi^{-2}\left(\frac{1}{\chi^{-1}-g_{\mathrm{D}}(k)}-\chi\right) \quad \text { and } \quad S_{\mathrm{gl}}^{(\mathrm{g})}=\chi^{-2} \frac{b}{\lambda_{1} \lambda_{2}}>0
$$

where (l) and (g) stand for liquid and gel. The X-ray scattering function $S$ covering both thermal fluctuations and static correlations, is equal in both cases, left unaffected by the introduction of crosslinks, whereas the glassy correlation function is nonzero only in the crosslinked sample. This is another indication that the fluctuations present at the time of crosslinking partially become frozen-in. (The thermal fluctuations $S_{\mathrm{th}}=S-S_{\mathrm{gl}}$ are diminished in equal measure.)

\section{Comparison to phenomenological theories}

In the following, we shall compare our correlation functions to the results of the phenomenological theories of de Gennes, Benhamou et al. and Read et al.. By analogy to polarisation in a dielectric medium, de Gennes [45] found the free energy of a crosslinked polymer blend to be given by

$$
f=\frac{1}{2} \sum_{\boldsymbol{k}}^{\prime}\left(\chi_{\mathrm{c}}-\chi_{\mathrm{m}}+k^{2}+\frac{C}{k^{2}}\right) \Psi_{\boldsymbol{k}} \Psi_{-\boldsymbol{k}}
$$

with a constant $C$ describing the rigidity of the gel. The free energy (3.109) reveals an instability at finite wavelength and thus predicts the transition to a microphase separated state. In scattering experiments, Briber and Bauer [49] confirmed this 
prediction but also found a nonzero scattering intensity at $k=0$, in disagreement with eq. (3.109), which they correctly attributed to the freezing-in of demixing fluctuations during preparation. To account for the preserved fluctuations disregarded in [45], Benhamou et al. [51, 52] complemented eq. (3.109) with a Debye-Hückel screening of the charges, the screening length $1 / \kappa$ determined such that the zero angle scattering intensity is not affected by the crosslinking:

$$
f=\frac{1}{2} \sum_{\boldsymbol{k}}^{\prime}\left(\chi_{\mathrm{c}}-\chi_{\mathrm{m}}+k^{2}+\frac{C}{\kappa^{2}+k^{2}}\right) \Psi_{\boldsymbol{k}} \Psi_{-\boldsymbol{k}}
$$

Our result, in the quadratic approximation and with the almost replica-symmetric ansatz $\Psi^{\alpha}=\left(1-\delta^{\alpha, 0}\right) \Psi$ for a gel prepared from a homogeneous liquid, reads

$$
\begin{aligned}
f= & \frac{1}{2} \sum_{\boldsymbol{k}}^{\prime}\left(\frac{1}{\chi_{\mathrm{m}}}-g_{\mathrm{D}}\left(k^{2}\right)+\mu(\mu-1) w\left(k^{2} /(\mu-1)\right)\right) \Psi_{\boldsymbol{k}} \Psi_{-\boldsymbol{k}} \\
& \approx \frac{1}{2 \chi_{\mathrm{m}}} \sum_{\boldsymbol{k}}^{\prime}\left(1-\chi_{\mathrm{m}}-\frac{\chi_{\mathrm{m}}}{3} k^{2}+\chi_{\mathrm{m}} \mu(\mu-1) w\left(k^{2} /(\mu-1)\right)\right) \Psi_{\boldsymbol{k}} \Psi_{-\boldsymbol{k}}
\end{aligned}
$$

see, e.g., eq. (3.83) or eq. (3.138). Obviously, the microscopic theory agrees with the phenomenological theory, apart from the shape of the localisation term assumed to be Lorentzian in eq. (3.110). The screening length and the elasticity constant can be unambiguously identified with the localisation length and the strength of the gel network, respectively, which are computed from our model without ad hoc assumptions.

An explicit account for the freezing-in of fluctuations due to the localisation of chains in the gel was first reported by Read et al. [53]. They considered a blend of homopolymers, anchored to randomly chosen points in space at both ends in an ad hoc approach to mimic the effect of crosslinking. Read et al. computed the volatile and persistent charge correlations

$$
S_{\mathrm{th}}(k)=\frac{1}{\chi_{\mathrm{c}}-\chi_{\mathrm{m}}+k^{2}-C / k^{2}}, \quad S_{\mathrm{gl}}(k)=\frac{C^{2} / k^{4} \cdot\left|\rho_{0 k}\right|^{2}}{\left(\chi_{\mathrm{c}}-\chi_{\mathrm{m}}+k^{2}-C / k^{2}\right)^{2}},
$$

with $\rho_{0 k}$ denoting the frozen-in charge density, indeed finding a nonzero value of $S_{\mathrm{gl}}(k)$ in the limit $k \rightarrow 0$. Close to microphase separation, the corresponding results of our theory are approximately given by

$$
\begin{aligned}
& S_{\mathrm{th}}(k) \propto \frac{1}{\chi_{\mathrm{c}}-\chi_{\mathrm{m}}+c_{2} \cdot\left(k^{2}-k_{\mathrm{c}}^{2}\right)^{2}} \\
& S_{\mathrm{gl}}(k) \propto \frac{\mu(\mu-1) w\left(k^{2} \xi_{1}^{2}\right)}{\left(\chi_{\mathrm{c}}-\chi_{\mathrm{m}}+c_{2} \cdot\left(k^{2}-k_{\mathrm{c}}^{2}\right)^{2}\right)^{2}}
\end{aligned}
$$

see eqs. (3.106) and (3.104); the constant $c_{2}$ is defined near eq. (3.104). The ad hoc treatment of gelation of ref. [53] and our microscopic description agree in predicting the glassy correlations to diverge twice as strongly as the thermal ones on approaching the microphase separation transition. 


\subsubsection{Microphase separation}

At $\chi_{\mathrm{m}}=\chi_{\mathrm{c}}$, the homogeneous gel ultimately becomes unstable with respect to phase separation. As we have seen in section 3.5.4, the instability first occurs for nonzero wavenumbers, indicating that the gel undergoes microscopic rather than macroscopic phase separation.

\section{Fourth order Landau expansion}

To discuss microphase separation, we require the Landau expansion of the effective free energy to fourth order in $\Psi$,

$$
\begin{aligned}
& n \mathcal{F}_{n}(\Psi, \tilde{\Omega}, \Omega)= \\
& \frac{N}{2} \sum_{\alpha=0}^{n} \sum_{\boldsymbol{k}}^{\prime}\left(\frac{1}{\chi^{\alpha}}-\frac{q^{2}}{\tilde{\lambda}^{\alpha}}-\left(1-q^{2}\right) g_{\mathrm{D}}\left(k^{2}\right)\right) \Psi_{\boldsymbol{k}}^{\alpha} \Psi_{-\boldsymbol{k}}^{\alpha}+\mathrm{i} q N \sum_{\alpha=0}^{n} \sum_{\boldsymbol{k}}^{\prime} \frac{1}{\tilde{\lambda}^{\alpha}} \Psi_{\boldsymbol{k}}^{\alpha} \tilde{\Omega}_{-\boldsymbol{k}}^{\alpha} \\
& +\frac{N}{2} \sum_{\alpha=0}^{n} \sum_{\boldsymbol{k}}^{\prime}\left(\frac{1}{\tilde{\lambda}^{\alpha}}+g_{\mathrm{D}}\left(k^{2}\right)\right) \tilde{\Omega}_{\boldsymbol{k}} \tilde{\Omega}_{-\boldsymbol{k}}+\frac{N}{2} \overline{\sum_{\hat{k}}}\left(\frac{V^{n}}{\mu}-g_{\mathrm{D}}\left(k^{2}\right)\right) \Omega_{\hat{k}} \Omega_{-\hat{k}} \\
& -\frac{N}{2}\left(1-q^{2}\right) \sum_{\alpha_{1} \neq \alpha_{2}} \sum_{\boldsymbol{k}_{1,2}}^{\prime} \overline{\sum_{\hat{k}_{3}}} g_{\Psi^{2} \Omega}\left(\boldsymbol{k}_{1}, \boldsymbol{k}_{2}\right) \Psi_{\boldsymbol{k}_{1}}^{\alpha_{1}} \Psi_{\boldsymbol{k}_{2}}^{\alpha_{2}} \Omega_{\hat{k}_{3}} \cdot \delta_{\boldsymbol{k}_{1} \otimes e_{\alpha_{1}}+\boldsymbol{k}_{2} \otimes e_{\alpha_{2}}+\hat{k}_{3}, \hat{0}} \\
& -\frac{N}{6} \sum_{\hat{k}_{1,2,3}} g_{3}\left(\hat{k}_{1}, \hat{k}_{2}\right) \Omega_{\hat{k}_{1}} \Omega_{\hat{k}_{2}} \Omega_{\hat{k}_{3}} \cdot \delta_{\hat{k}_{1}+\hat{k}_{2}+\hat{k}_{3}, \hat{0}} \\
& +\frac{N}{3} q\left(1-q^{2}\right) \sum_{\alpha=0}^{n} \sum_{\boldsymbol{k}_{1,2,3}}^{\prime} g_{3}\left(\boldsymbol{k}_{1}, \boldsymbol{k}_{2}\right) \Psi_{\boldsymbol{k}_{1}}^{\alpha} \Psi_{\boldsymbol{k}_{2}}^{\alpha} \Psi_{\boldsymbol{k}_{3}}^{\alpha} \cdot \delta_{\boldsymbol{k}_{1}+\boldsymbol{k}_{2}+\boldsymbol{k}_{3}, \mathbf{0}} \\
& -\frac{\mathrm{i} N}{2}\left(1-q^{2}\right) \sum_{\alpha=0}^{n} \sum_{\boldsymbol{k}_{1,2,3}}^{\prime} g_{3}\left(\boldsymbol{k}_{1}, \boldsymbol{k}_{2}\right) \Psi_{\boldsymbol{k}_{1}}^{\alpha} \Psi_{\boldsymbol{k}_{2}}^{\alpha} \tilde{\Omega}_{\boldsymbol{k}_{3}}^{\alpha} \cdot \delta_{\boldsymbol{k}_{1}+\boldsymbol{k}_{2}+\boldsymbol{k}_{3}, \mathbf{0}} \\
& -\frac{N}{24}\left(1-q^{2}\right)\left(1+3 q^{2}\right) \sum_{\alpha=0}^{n} \sum_{\boldsymbol{k}_{1,2,3,4}}^{\prime} g_{\Psi^{4}}\left(\boldsymbol{k}_{1}, \boldsymbol{k}_{2}, \boldsymbol{k}_{3}\right) \Psi_{\boldsymbol{k}_{1}}^{\alpha} \Psi_{\boldsymbol{k}_{2}}^{\alpha} \Psi_{\boldsymbol{k}_{3}}^{\alpha} \Psi_{\boldsymbol{k}_{4}}^{\alpha} \cdot \delta_{\boldsymbol{k}_{1}+\boldsymbol{k}_{2}+\boldsymbol{k}_{3}+\boldsymbol{k}_{4}, \mathbf{0}} \\
& +\frac{N}{8}\left(1-q^{2}\right)\left(1+3 q^{2}\right) \sum_{\alpha=0}^{n}\left(\sum_{\boldsymbol{k}}^{\prime} g_{\mathrm{D}}\left(k^{2}\right) \Psi_{\boldsymbol{k}}^{\alpha} \Psi_{-\boldsymbol{k}}^{\alpha}\right)^{2} \\
& +\mathcal{O}\left(\Psi^{5}, \tilde{\Omega}^{3}, \Omega^{4}, \Psi^{3} \tilde{\Omega}, \Psi^{3} \Omega, \Psi^{2} \Omega^{2}, \tilde{\Omega}^{2} \Omega\right)
\end{aligned}
$$

with $g_{\Psi^{4}}$ defined in appendix D.2.1. In analogy to section 2.5.4, we integrate out the density field $\tilde{\Omega}$ on the saddle point level to second order in $\Psi$, where

$$
\begin{aligned}
& \overline{\tilde{\Omega}}_{\boldsymbol{k}}^{\alpha}=\frac{-\mathrm{i} q}{1+\tilde{\lambda}^{\alpha} g_{\mathrm{D}}\left(k^{2}\right)} \Psi_{\boldsymbol{k}}^{\alpha} \\
& \quad+\frac{\mathrm{i}\left(1-q^{2}\right)}{2\left(1 / \tilde{\lambda}^{\alpha}+g_{\mathrm{D}}\left(k^{2}\right)\right)} \sum_{\boldsymbol{k}_{\boldsymbol{2}, 3}}^{\prime} g_{3}\left(\boldsymbol{k}_{2}, \boldsymbol{k}_{3}\right) \Psi_{\boldsymbol{k}_{2}}^{\alpha} \Psi_{\boldsymbol{k}_{3}}^{\alpha} \cdot \delta_{\boldsymbol{k}+\boldsymbol{k}_{2}+\boldsymbol{k}_{3}, \mathbf{0}}
\end{aligned}
$$


For low compressibility or in the symmetric case we have $\tilde{\Omega} \sim \Psi^{2}$, so that the expansion to second order in $\tilde{\Omega}$ and to fourth order in $\Psi$ is consistent. As the physical density is proportional to $1 /\left(\lambda_{\mathrm{m}}-\mu\right)$ and thus vanishes in the incompressible limit for any $\tilde{\Omega}$, the nonzero saddle point value is not in contradiction with this limit. For a discussion of eq. (3.116), see section 3.5.9.

We restrict the discussion to weak gels just beyond the transition, i.e. $\mu-1 \ll 1$ and $\chi-\chi_{\mathrm{c}} \ll 1$, so both $\bar{\Psi}$ and $\bar{\Omega}$ are small. In the vicinity of the microphase transition, the second-order coefficient becomes very small and is strongly affected by the coupling of $\Psi$ and $\Omega$, despite of the smallness of the latter. In contrast, the coefficients of the third-order term (if present at all) and the fourth-order term are of the order one, so that the coupling is negligible and we can let $\bar{\Omega}=0$ therein. This allows us to disregard the effect of $\bar{\Psi}$ on $\bar{\Omega}$ : a shift in $\bar{\Omega}$ would be of the order $\Psi^{2}$ and contribute only to fourth in $\Psi$, where the influence of gelation can be neglected anyway. Hence we can approximate $\bar{\Omega}$ by eq. (3.81). Inserting the saddle points of $\tilde{\Omega}$ and $\Omega$ into eq. (3.115) we obtain

$$
\begin{aligned}
& n \mathcal{F}_{n}(\Psi):=n \mathcal{F}_{n}(\Psi, \bar{\Omega}, \bar{\Omega})= \\
& \frac{N}{2} \sum_{\alpha_{1,2}=0}^{n} \sum_{\boldsymbol{k}}^{\prime}\left(\left(1-q^{2}\right) A_{\alpha_{1} \alpha_{2}}(k)-\frac{q^{2} g_{\mathrm{D}}\left(k^{2}\right) \cdot \delta_{\alpha_{1}, \alpha_{2}}}{\tilde{\lambda}^{\alpha_{1}}\left(1+\tilde{\lambda}^{\alpha_{1}} g_{\mathrm{D}}\left(k^{2}\right)\right)}\right) \Psi_{\boldsymbol{k}}^{\alpha_{2}} \Psi_{-\boldsymbol{k}}^{\alpha_{2}} \\
& +\frac{q\left(1-q^{2}\right) N}{3} \sum_{\alpha=0}^{n} \sum_{\boldsymbol{k}_{1,2,3}}^{\prime} g_{3}\left(\boldsymbol{k}_{1}, \boldsymbol{k}_{2}\right)\left(1-\frac{3 g_{3}\left(\boldsymbol{k}_{1}, \boldsymbol{k}_{2}\right)}{2\left(1+\tilde{\lambda}^{\alpha}\right)}\right) \Psi_{\boldsymbol{k}_{1}}^{\alpha} \Psi_{\boldsymbol{k}_{2}}^{\alpha} \Psi_{\boldsymbol{k}_{3}}^{\alpha} \cdot \delta_{\boldsymbol{k}_{1}+\boldsymbol{k}_{2}+\boldsymbol{k}_{3}, \mathbf{0}} \\
& +\frac{\left(1-q^{2}\right) N}{8} \sum_{\alpha=0}^{n} \sum_{\boldsymbol{k}_{1,2,3,4}}^{\prime}\left(\frac{\left(1-q^{2}\right) g_{3}\left(\boldsymbol{k}_{1}, \boldsymbol{k}_{2}\right) g_{3}\left(\boldsymbol{k}_{3}, \boldsymbol{k}_{4}\right)}{g_{\mathrm{D}}\left(\left(\boldsymbol{k}_{1}+\boldsymbol{k}_{2}\right)^{2}\right)}\left(1-\frac{1}{1+\tilde{\lambda}^{\alpha}}\right)\right. \\
& \left.\quad-\frac{1+3 q^{2}}{3} g_{\Psi^{4}}\left(\boldsymbol{k}_{1}, \boldsymbol{k}_{2}, \boldsymbol{k}_{3}\right)\right) \Psi_{\boldsymbol{k}_{1}}^{\alpha} \Psi_{\boldsymbol{k}_{2}}^{\alpha} \Psi_{\boldsymbol{k}_{3}}^{\alpha} \Psi_{\boldsymbol{k}_{4}}^{\alpha} \cdot \delta_{\boldsymbol{k}_{1}+\boldsymbol{k}_{2}+\boldsymbol{k}_{3}+\boldsymbol{k}_{4}, \mathbf{0}} \\
& \quad+\frac{\left(1-q^{2}\right) N}{2} \sum_{\alpha=0}^{n}\left(q^{2}+\frac{1-q^{2}}{4\left(1+\tilde{\lambda}^{\alpha}\right)}\right)\left(\sum_{\boldsymbol{k}}^{\prime} g_{\mathrm{D}}\left(k^{2}\right) \Psi_{\boldsymbol{k}}^{\alpha} \Psi_{-\boldsymbol{k}}^{\alpha}\right)^{2}
\end{aligned}
$$

with the matrix elements $A_{\alpha_{1} \alpha_{2}}$ of $\mathbf{A}$ defined in eq. (3.84). Some of the terms due to tracing out $\tilde{\Omega}$ survive the incompressible limit $\tilde{\lambda}^{\alpha} \rightarrow \infty$, yielding additional fourth-order terms in $\Psi$ to the effective free energy, which in this limit is given by

$$
\begin{aligned}
& \frac{n}{\left(1-q^{2}\right) N} \mathcal{F}_{n}(\Psi)= \\
& \frac{1}{2} \sum_{\alpha_{1,2}=0}^{n} \sum_{\boldsymbol{k}}^{\prime} A_{\alpha_{1} \alpha_{2}}(k) \Psi_{\boldsymbol{k}}^{\alpha_{2}} \Psi_{-\boldsymbol{k}}^{\alpha_{2}}+\frac{q}{3} \sum_{\alpha=0}^{n} \sum_{\boldsymbol{k}_{1,2,3}}^{\prime} g_{3}\left(\boldsymbol{k}_{1}, \boldsymbol{k}_{2}\right) \Psi_{\boldsymbol{k}_{1}}^{\alpha} \Psi_{\boldsymbol{k}_{2}}^{\alpha} \Psi_{\boldsymbol{k}_{3}}^{\alpha} \cdot \delta_{\boldsymbol{k}_{1}+\boldsymbol{k}_{2}+\boldsymbol{k}_{3}, \mathbf{0}} \\
& \quad+\frac{1}{8} \sum_{\alpha=0}^{n} \sum_{\boldsymbol{k}_{1,2,3,4}}^{\prime}\left(\frac{\left(1-q^{2}\right) g_{3}\left(\boldsymbol{k}_{1}, \boldsymbol{k}_{2}\right) g_{3}\left(\boldsymbol{k}_{3}, \boldsymbol{k}_{4}\right)}{g_{\mathrm{D}}\left(\left(\boldsymbol{k}_{1}+\boldsymbol{k}_{2}\right)^{2}\right)}-\frac{1+3 q^{2}}{3} g_{\Psi^{4}}\left(\boldsymbol{k}_{1}, \boldsymbol{k}_{2}, \boldsymbol{k}_{3}\right)\right) \\
& \times \Psi_{\boldsymbol{k}_{1}}^{\alpha} \Psi_{\boldsymbol{k}_{2}}^{\alpha} \Psi_{\boldsymbol{k}_{3}}^{\alpha} \Psi_{\boldsymbol{k}_{4}}^{\alpha} \cdot \delta_{\boldsymbol{k}_{1}+\boldsymbol{k}_{2}+\boldsymbol{k}_{3}+\boldsymbol{k}_{4}, \mathbf{0}}
\end{aligned}
$$




$$
+\frac{q^{2}}{2} \sum_{\alpha=0}^{n}\left(\sum_{k}^{\prime} g_{\mathrm{D}}\left(k^{2}\right) \Psi_{k}^{\alpha} \Psi_{-\boldsymbol{k}}^{\alpha}\right)^{2}
$$

\section{Symmetric case: lamellar phases}

We first discuss of microphase separation for the symmetric case $q=0$, where the third-order term and the biquadratic term in eq. (3.118) vanish. The remaining fourth-order term depends on the separation pattern. We consider a simple lamellar microphase state with sinusoidal modulations in real space,

$$
\bar{\Psi}_{\boldsymbol{k}^{\prime}}^{\alpha}= \begin{cases}0, & \text { for } \alpha=0 \\ \left(\delta_{\boldsymbol{k}^{\prime}, \boldsymbol{k}}+\delta_{\boldsymbol{k}^{\prime},-\boldsymbol{k}}\right) \psi, & \text { otherwise }\end{cases}
$$

which is replica-symmetric apart from the zeroth replica reflecting the preparation ensemble. The wavelength $2 \pi / k$ and the amplitude $\psi \geq 0$ are variational parameters subject to optimisation. Other simple structures, viz. hexagonally ordered cylinders and spheres on a bcc lattice, lead to higher free energies; the same holds for superpositions of sinusoidal charge density modulations like eq. (3.119) having equal wavelength but random orientations. As will be shown in sections 3.5.8 and 3.5.9, these conclusions, however, depend on the symmetry and compressibility of the blend. Inserting the ansatz (3.119) into eq. (3.118) yields

$$
2 \cdot f(k, \psi):=\frac{2}{N} \cdot \lim _{n \rightarrow 0} \mathcal{F}_{n}(\{\Psi\}) \approx 2 \lambda_{2}(k) \cdot \psi^{2}+g_{4}\left(k^{2}\right) \cdot \psi^{4}
$$

with $\lambda_{2}$ defined in eq. (3.88) and

$$
g_{4}\left(k^{2}\right):=\frac{1}{2}\left(\frac{\left(g_{3}\left(k^{2}\right)\right)^{2}}{g_{\mathrm{D}}\left(4 k^{2}\right)}+2\left(g_{\mathrm{D}}\left(k^{2}\right)\right)^{2}-g_{\Psi^{4}}\left(k^{2}\right)\right)=1-\frac{2}{3} k^{2}+\mathcal{O}\left(k^{4}\right) .
$$

At the onset of the microphase separation, the optimal wavenumber is given by $k_{\mathrm{c}}$ defined in eq. (3.90), and the amplitude is infinitely small. For $\chi_{\mathrm{m}}>\chi_{\mathrm{c}}$, amplitude and wavelength must be determined by variational optimisation of $f$, which can be done analytically for $\psi$, leading to

$$
\psi_{\min }^{2}(k)=-\frac{\lambda_{2}(k)}{g_{4}\left(k^{2}\right)} \quad \text { and } \quad f\left(k, \psi_{\min }\right)=-\frac{\left(\lambda_{2}(k)\right)^{2}}{2 g_{4}\left(k^{2}\right)}
$$

To determine the optimal wavenumber we expand $\frac{\partial f\left(k, \psi_{\min }\right)}{\partial\left(k^{2}\right)}=0$ about $k^{2}=k_{\mathrm{c}}^{2}$ and obtain

$$
k_{\min }^{2}-k_{\mathrm{c}}^{2}=\left.\frac{-\frac{\partial \ln g_{4}\left(k^{2}\right)}{\partial\left(k^{2}\right)}}{2 \frac{\partial^{2} \lambda_{2}(k)}{\partial\left(k^{2}\right)^{2}}}\right|_{k=k_{\mathrm{c}}} \cdot \frac{\chi_{\mathrm{m}}-\chi_{\mathrm{c}}}{\chi_{\mathrm{m}} \chi_{\mathrm{c}}}+\mathcal{O}\left(\left(\chi_{\mathrm{m}}-\chi_{\mathrm{c}}\right)^{2}\right) .
$$


For small $\mu-1$, we can expand the proportionality constant in powers of $(\mu-1)$, which finally leads to

$$
\begin{aligned}
k_{\min }^{2}-k_{\mathrm{c}}^{2} & =\frac{-\left.\frac{\partial \ln g_{4}\left(k^{2}\right)}{\partial\left(k^{2}\right)}\right|_{k=0}}{2 \cdot w^{\prime \prime}\left(\lim _{\mu \rightarrow 1} \frac{k_{\mathrm{c}}^{2}}{\mu-1}\right)} \cdot\left(\chi_{\mathrm{m}}-\chi_{\mathrm{c}}\right)(\mu-1)+\ldots \\
& \approx 1.09 \cdot\left(\chi_{\mathrm{m}}-\chi_{\mathrm{c}}\right)(\mu-1)+\mathcal{O}\left(\left(\chi_{\mathrm{m}}-\chi_{\mathrm{c}}\right)^{2},(\mu-1)^{2}\right),
\end{aligned}
$$

in agreement with numerical results. The optimal amplitude is given by $\psi_{\min }\left(k_{\min }\right)$, which is zero at the microphase transition. An expansion in powers of $\chi_{\mathrm{m}}-\chi_{\mathrm{c}}$ (and $\mu-1$ ) yields

$$
\begin{aligned}
\psi_{\min }^{2} & =\frac{\chi_{\mathrm{m}}-\chi_{\mathrm{c}}}{\chi_{\mathrm{c}}^{2} g_{4}\left(k_{\mathrm{c}}\right)}+\mathcal{O}\left(\left(\chi_{\mathrm{m}}-\chi_{\mathrm{c}}\right)^{2}\right) \\
& =\left(\chi_{\mathrm{m}}-\chi_{\mathrm{c}}\right)+\mathcal{O}\left(\left(\chi_{\mathrm{m}}-\chi_{\mathrm{c}}\right)^{2},(\mu-1)\right)
\end{aligned}
$$

Hence, we find a second-order phase transition, with the squared amplitude of the microphase separation pattern, as well as the shift of inverse square of the spatial period, depending linearly on the distance $\left(T_{\mathrm{c}}-T\right)$ to the transition. (The functional form of the period should, however, be taken with a pinch of salt, since it strongly depends on the detailed choice of the model through the correlation function $g_{4}(k)$.)

\subsubsection{Effects of compositional asymmetry}

As we have seen in sections 3.5.2, 3.5.4 and 3.5.6, an asymmetric composition $q \neq 0$ just leads to a rescaling of the spinodals $\chi_{\mathrm{p}}=1 / \gamma, \chi_{\mathrm{m}}=\chi_{\mathrm{c}}$ and the charge correlations $S, S_{\mathrm{gl}}, S_{\mathrm{th}}$. However, due to the third-order term proportional to $q$ in the Landau free energy (3.118), both the macrophase separation of the uncrosslinked liquid and the microphase separation of the gel become first-order transitions for $q \neq 0$, and the spinodals no longer coincide with the equilibrium phase transition point.

In the following we shall discuss the effect of asymmetry on the equilibrium microphase transition point $\chi_{\mathrm{t}} \leq \chi_{\mathrm{c}}$. Since within mean-field theory, the microphase transition is unaffected by frozen fluctuations, the incompatibility $\chi_{\mathrm{p}}$ prior to preparation is nearly irrelevant. The only constraint is that the gel is required to be prepared from a homogeneous mixture, no matter whether this is an undercooled or the equilibrium state.

The typical domain size of the microphases is more or less given by the localisation length $\xi_{1}$ of the gel, so we can drop the $k$-dependence of the higher-order terms in the Landau free energy. With the ansatz $\Psi_{\boldsymbol{k}}^{\alpha}=\left(1-\delta_{\alpha, 0}\right) \Psi_{\boldsymbol{k}}$ corresponding to the mixed state in the preparation ensemble and replica-symmetric phase separation 
in the measurement ensemble, this leads to the rescaled free energy density

$$
\begin{aligned}
& f(\{\Psi\}):=\lim _{n \rightarrow 0} \frac{\mathcal{F}_{n}(\{\Psi\})}{N\left(1-q^{2}\right)} \\
& =\frac{1}{2} \sum_{\boldsymbol{k}}^{\prime} \lambda_{2}(k) \Psi_{\boldsymbol{k}} \Psi_{-\boldsymbol{k}}+\frac{q}{3} \sum_{\boldsymbol{k}_{1,2,3}}^{\prime} \Psi_{\boldsymbol{k}_{1}} \Psi_{\boldsymbol{k}_{2}} \Psi_{\boldsymbol{k}_{3}} \cdot \delta_{\boldsymbol{k}_{1}+\boldsymbol{k}_{2}+\boldsymbol{k}_{3}, \mathbf{0}} \\
& +\frac{q^{2}}{2}\left(\sum_{\boldsymbol{k}}^{\prime} \Psi_{\boldsymbol{k}} \Psi_{-\boldsymbol{k}}\right)^{2}+\frac{1-3 q^{2}}{12} \sum_{\boldsymbol{k}_{1,2,3,4}}^{\prime} \Psi_{\boldsymbol{k}_{1}} \Psi_{\boldsymbol{k}_{2}} \Psi_{\boldsymbol{k}_{3}} \Psi_{\boldsymbol{k}_{4}} \cdot \delta_{\boldsymbol{k}_{1}+\boldsymbol{k}_{2}+\boldsymbol{k}_{3}+\boldsymbol{k}_{4}, \mathbf{0}}
\end{aligned}
$$

with $\lambda_{2}(k)$ defined in eq. (3.88).

Besides the lamellar microphases discussed in section 3.5.7, we now consider two additional morphologies: Cylindrical phases with parallel orientation, aligned on a honeycomb lattice in the perpendicular plane, and spherical domains on a body centred cubic lattice. The three morphologies are shown in fig. B.1. They are chosen because they are known to appear in the microphase separation of regular block copolymer melts [3, 6]. Although a randomly crosslinked blend will probably reveal only local order, the regular structures are useful to construct a simple and tractable ansatz for the microphase separated state:

$$
\Psi_{\boldsymbol{k}^{\prime}}=\frac{\Psi}{\sqrt{m}} \sum_{i=1}^{m}\left(\delta_{\boldsymbol{k}^{\prime}, k \boldsymbol{n}_{i}}+\delta_{\boldsymbol{k}^{\prime},-k \boldsymbol{n}_{i}}\right)
$$

with the lattice vectors $\left\{\boldsymbol{n}_{i}\right\}$ defined in appendix B.1 and $m=1$ for lamellæ, $m=3$ for hexagonally ordered cylinders and $m=6$ for spheres on a bcc lattice. The wavenumber $k$ and the amplitude $\Psi$ of the charge density modulations have to be determined by minimisation of the effective free energy. The optimal wave obviously is given by $k_{\mathrm{c}}$ defined in eq. (3.90), so we can make use of

$$
\lambda_{2}\left(k_{\mathrm{c}}\right)=\frac{1}{\left(1-q^{2}\right) \chi_{\mathrm{m}}}-\frac{1}{\left(1-q^{2}\right) \chi_{\mathrm{c}}} .
$$

With the lattice ansatz (3.127), the evaluation of the higher-order sums in eq. (3.126) amounts to counting the number of possible "loops" of two, three and four lattice vectors that add to zero; this is carried out in appendix B.1. We have $\sum_{\boldsymbol{k}}^{\prime} \Psi_{\boldsymbol{k}} \Psi_{-\boldsymbol{k}}=2 \Psi^{2}$, independent of the morphology, and

$$
\begin{aligned}
& \sum_{\boldsymbol{k}_{1,2,3}}^{\prime} \Psi_{\boldsymbol{k}_{1}} \Psi_{\boldsymbol{k}_{2}} \Psi_{\boldsymbol{k}_{3}} \cdot \delta_{\boldsymbol{k}_{1}+\boldsymbol{k}_{2}+\boldsymbol{k}_{3}, \mathbf{0}}=c_{3}^{(m)} \Psi^{3}, \\
& \sum_{\boldsymbol{k}_{1,2,3,4}}^{\prime} \Psi_{\boldsymbol{k}_{1}} \Psi_{\boldsymbol{k}_{2}} \Psi_{\boldsymbol{k}_{3}} \Psi_{\boldsymbol{k}_{4}} \cdot \delta_{\boldsymbol{k}_{1}+\boldsymbol{k}_{2}+\boldsymbol{k}_{3}+\boldsymbol{k}_{4}, \mathbf{0}}=c_{4}^{(m)} \Psi^{4}
\end{aligned}
$$

where

$$
\begin{aligned}
& c_{3}^{(1)}=0, \quad c_{3}^{(3)}=4 / \sqrt{3}, \quad c_{3}^{(6)}=4 \cdot \sqrt{2 / 3}, \\
& c_{4}^{(1)}=6, \quad c_{4}^{(3)}=10, \quad c_{4}^{(6)}=15 .
\end{aligned}
$$


Thus, the free energy density becomes

$$
f(\Psi):=\frac{\chi_{\mathrm{m}}^{-1}-\chi_{\mathrm{c}}^{-1}}{1-q^{2}} \cdot \Psi^{2}+\frac{q c_{3}^{(m)}}{3} \cdot \Psi^{3}+\left(2 q^{2}+\frac{\left(1-3 q^{2}\right) c_{4}^{(m)}}{12}\right) \cdot \Psi^{4}
$$

In the symmetric case, the free energy (3.133) is minimised by the lamellar ansatz, which corresponds to the smallest $c_{4}$. For $m=1$, the third-order term vanishes even if $q \neq 0$, so the transition remains second order and the spinodal indeed indicates the equilibrium phase transition point with respect to lamellæ. For cylinders and bcc spheres, in contrast, the equilibrium transition point $\chi_{\mathrm{t}}$ is shifted according to

$$
\begin{aligned}
& \frac{1}{\left(1-q^{2}\right) \chi_{\mathrm{t}}}-\frac{1}{\left(1-q^{2}\right) \chi_{\mathrm{c}}}=\frac{\left(q c_{3}^{(m)} / 3\right)^{2}}{4\left(2 q^{2}+\left(1-3 q^{2}\right) c_{4}^{(m)} / 12\right)}=\frac{\left(q c_{3}^{(m)}\right)^{2}}{72 q^{2}+3\left(1-3 q^{2}\right) c_{4}^{(m)}} \\
& =\left\{\begin{array}{l}
\frac{8 q^{2}}{45-27 q^{2}}=\frac{24}{135} q^{2}+\mathcal{O}\left(q^{4}\right) \text { for } m=3 \text { (cylinders) } \\
\frac{32 q^{2}}{135-189 q^{2}}=\frac{32}{135} q^{2}+\mathcal{O}\left(q^{4}\right) \text { for } m=6 \text { (bcc spheres). }
\end{array}\right.
\end{aligned}
$$

In the asymmetric case, the bcc spheres yield the lowest equilibrium transition point of the three possibilities possibilities, i.e. microphases first occur with bcc symmetry. This is to be expected, as the lowest surface to volume ratio of the minority phase is minimal for spherical domains, and it is in agreement with the finding of Alexander and McTague [86] of a general preference for bcc symmetry in crystal nucleation. It should be noted that, for large $q^{2}$, the right hand side in the last line of eq. (3.134) diverges; in this case the transition is strongly first order, and the Landau expansion breaks down.

\subsubsection{Effects of compressibility}

The influence of compressibility on the phase separation behaviour becomes apparent in the saddle point (3.116) of the density field $\tilde{\Omega}$, which takes a nonzero value in a phase separated state. For simplicity we restrict the discussion of compressibility effects to the symmetric case, where the shifted and the original density fields coincide. In this case eq. (3.116) reduces to

$$
\bar{\Omega}_{\boldsymbol{k}}^{\alpha} \equiv \overline{\tilde{\Omega}}_{\boldsymbol{k}}^{\alpha}=\frac{\mathrm{i}}{2\left(1 / \tilde{\lambda}^{\alpha}+g_{\mathrm{D}}\left(k^{2}\right)\right)} \sum_{\boldsymbol{k}_{2,3}}^{\prime} g_{3}\left(\boldsymbol{k}_{2}, \boldsymbol{k}_{3}\right) \Psi_{\boldsymbol{k}_{2}}^{\alpha} \Psi_{\boldsymbol{k}_{3}}^{\alpha} \cdot \delta_{\boldsymbol{k}+\boldsymbol{k}_{2}+\boldsymbol{k}_{3}, \mathbf{0}}
$$

As described by eq. (3.135), a compressible system can avoid unfavourable $A-B$ contacts and lower its energy by diluting mixed regions having many such contacts, which are characterised by a small absolute charge density, and condensing regions that are rich in either $A$ or $B$, which have a high absolute charge density. For 
Figure 3.7: Coupling of mass and charge density $\Omega$ and $\Psi$. Zones of large absolute charge density are condensed, and hence the mass density is modulated with half the wavelength of the charge density modulations.

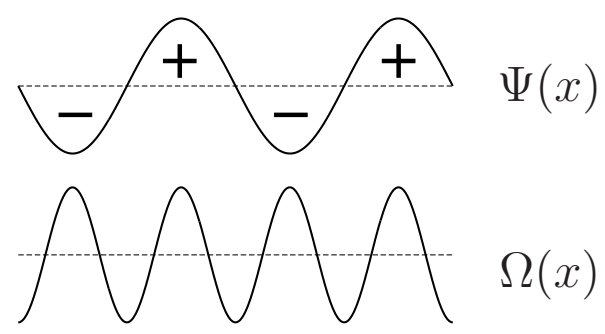

example, in the case of lamellar microphases described by a single wavevector $\boldsymbol{k}$ as in eq. (3.119), eq. (3.135) predicts density field modulations having wavevector $\boldsymbol{k}_{1}= \pm 2 \boldsymbol{k}$, i.e. with twice the wavenumber of the charge density modulations. This is intuitively clear: Along one spatial period of the charge density modulations, their modulus or square, and thus the mass density, oscillates twice, corresponding to the half wavelength or the double wavenumber; this is illustrated in fig. 3.7.

The saddle point of $\tilde{\Omega}$ does not vanish for $\mu \rightarrow \infty$. As pointed out before, this is not in contradiction with incompressibility, since the physical density is given by $Q_{\boldsymbol{k}} \sim\left(\tilde{\Omega}_{\boldsymbol{k}}+\mathrm{i} q \Psi\right) /\left(\lambda_{\mathrm{m}}-\mu\right)$ and vanishes in any case for $\lambda_{\mathrm{m}} \rightarrow \infty$. While the physical density becomes infinitely small in the incompressible limit, the bulk modulus diverges. The effect of the quadratic coupling described above therefore survives the limit, contributing additional fourth-order terms in $\Psi$ to the Landau expansion.

To study microphase separation in the symmetric but compressible case, we let $q=0$ in eq. (3.117). Furthermore, we drop the $k$ dependence in the higher-order vertices, since in the approximation, the domain size is determined by $k_{\mathrm{c}}$ or the localisation length of the gel. (The $k$-dependence is only necessary to compute the domain size deeper in the phase separated state.) Thus we have

$$
\begin{aligned}
n \mathcal{F}_{n}(\Psi) & =\frac{N}{2} \sum_{\alpha_{1,2}=0}^{n} \sum_{\boldsymbol{k}}^{\prime} A_{\alpha_{1} \alpha_{2}}(k) \Psi_{\boldsymbol{k}}^{\alpha_{2}} \Psi_{-\boldsymbol{k}}^{\alpha_{2}}+\frac{N}{8} \sum_{\alpha=0}^{n} \frac{1}{1+\tilde{\lambda}^{\alpha}}\left(\sum_{\boldsymbol{k}}^{\prime} \Psi_{\boldsymbol{k}}^{\alpha} \Psi_{-\boldsymbol{k}}^{\alpha}\right)^{2} \\
+ & \frac{N}{12} \sum_{\alpha=0}^{n} \sum_{\boldsymbol{k}_{1,2,3,4}}^{\prime}\left(1-\frac{3}{2\left(1+\tilde{\lambda}^{\alpha}\right)}\right) \Psi_{\boldsymbol{k}_{1}}^{\alpha} \Psi_{\boldsymbol{k}_{2}}^{\alpha} \Psi_{\boldsymbol{k}_{3}}^{\alpha} \Psi_{\boldsymbol{k}_{4}}^{\alpha} \cdot \delta_{\boldsymbol{k}_{1}+\boldsymbol{k}_{2}+\boldsymbol{k}_{3}+\boldsymbol{k}_{4}, \mathbf{0}}
\end{aligned}
$$

With the ansatz $\Psi^{\alpha}=\left(1-\delta_{\alpha, 0}\right) \Psi$, and with

$$
\lambda_{\mathrm{eff}}:=1+\tilde{\lambda}^{\alpha}=\lambda_{\mathrm{m}}+1-\mu / V^{n} \approx \lambda_{\mathrm{m}} \quad \text { for } \alpha \geq 1,
$$

the effective free energy simplifies to

$$
\begin{aligned}
f(\Psi, k):=\lim _{n \rightarrow 0} \frac{1}{N} \mathcal{F}_{n}(\Psi)=\frac{1}{2} \sum_{\boldsymbol{k}}^{\prime} \lambda_{2}(k) \Psi_{\boldsymbol{k}} \Psi_{-\boldsymbol{k}}+\frac{1}{8 \lambda_{\mathrm{eff}}}\left(\sum_{\boldsymbol{k}}^{\prime} \Psi_{\boldsymbol{k}} \Psi_{-\boldsymbol{k}}\right)^{2} \\
+\frac{1}{12}\left(1-\frac{3}{2 \lambda_{\mathrm{eff}}}\right) \sum_{\boldsymbol{k}_{1,2,3,4}}^{\prime} \Psi_{\boldsymbol{k}_{1}} \Psi_{\boldsymbol{k}_{2}} \Psi_{\boldsymbol{k}_{3}} \Psi_{\boldsymbol{k}_{4}} \cdot \delta_{\boldsymbol{k}_{1}+\boldsymbol{k}_{2}+\boldsymbol{k}_{3}+\boldsymbol{k}_{4}, \mathbf{0}}
\end{aligned}
$$


To account for the potential randomness of the microphase pattern, we extend the lamellar ansatz of section 3.5 .7 by allowingfor a superposition of $Z$ onedimensional waves, each with identical wavenumber $k$ but random phase $\Phi_{z}$ and wavevector orientation $\boldsymbol{n}_{z}$, i.e.,

$$
\Psi_{\boldsymbol{k}^{\prime}}=\frac{\Psi}{\sqrt{Z}} \sum_{z=1}^{Z}\left(\mathrm{e}^{\mathrm{i} \Phi_{z}} \delta_{\boldsymbol{k}^{\prime},-k \boldsymbol{n}_{z}}+\mathrm{e}^{-\mathrm{i} \Phi_{z}} \delta_{\boldsymbol{k}^{\prime}, k \boldsymbol{n}_{z}}\right)
$$

corresponding to $2 \Psi /\left(V Z^{1 / 2}\right) \cdot \sum_{z=1}^{Z} \cos \left(k \boldsymbol{n}_{z} \boldsymbol{k}^{\prime}+\Phi_{z}\right)$ in real space. The optimal number of orientations will be determined later. A few examples of such random morphologies are shown in fig. 3.8, the number of phases ranging from 1 to 1000 .

We assume that none of the orientations $\boldsymbol{n}_{z}$ are collinear, and thus the quadratic sums in eq. (3.138) yield

$$
\sum_{\boldsymbol{k}^{\prime}}^{\prime} \lambda_{2}\left(k^{\prime}\right) \Psi_{\boldsymbol{k}^{\prime}} \Psi_{-\boldsymbol{k}^{\prime}}=2 \lambda_{2}(k) \Psi^{2} \quad \text { and } \quad \sum_{\boldsymbol{k}^{\prime}}^{\prime} \Psi_{\boldsymbol{k}^{\prime}} \Psi_{-\boldsymbol{k}^{\prime}}=2 \Psi^{2} .
$$

Computing the fourth-order sum in eq. (3.138) again amounts to counting the number of possible closed loops of orientations. Because of the randomness, the existence of quadruples of orientations able to form a closed loop is very unlikely, except for the degenerate planar case of pairs of opposite vectors $\left( \pm \boldsymbol{n}_{z}, \pm \boldsymbol{n}_{z^{\prime}}\right)$, and hence we disregard non-planar loops. Single orientations allow for the construction of quadruples $\left(\boldsymbol{n}_{z}, \boldsymbol{n}_{z},-\boldsymbol{n}_{z},-\boldsymbol{n}_{z}\right)$ that can be ordered in $\left(\begin{array}{l}4 \\ 2\end{array}\right)=6$ ways. Quadruples $\left( \pm \boldsymbol{n}_{z}, \pm \boldsymbol{n}_{z^{\prime}}\right)$ of two pairs of different orientations can be ordered in 4 ! $=24$ different ways, and there are $\frac{1}{2} Z(Z-1)$ such pairs. Thus, the quartic sum in eq. (3.138) yields

$$
\begin{aligned}
& \sum_{\boldsymbol{k}_{1,2,3,4}}^{\prime} \Psi_{\boldsymbol{k}_{1}} \Psi_{\boldsymbol{k}_{2}} \Psi_{\boldsymbol{k}_{3}} \Psi_{\boldsymbol{k}_{4}} \cdot \delta_{\boldsymbol{k}_{1}+\boldsymbol{k}_{2}+\boldsymbol{k}_{3}+\boldsymbol{k}_{4}, \mathbf{0}}=\frac{12 Z(Z-1)+6 Z}{Z^{2}} \Psi^{4} \\
&=12\left(1-\frac{1}{2 Z}\right) \Psi^{4}
\end{aligned}
$$

Inserting the sums into the free energy density we obtain

$$
f(\Psi, k)=\lambda_{2}(k) \cdot \Psi^{2}+\left(\left(1-\frac{1}{\lambda_{\text {eff }}}\right)-\left(1-\frac{3}{2 \lambda_{\text {eff }}}\right) \frac{1}{2 Z}\right) \cdot \Psi^{4} .
$$

As expected, the minimisation of $f$ with respect to $k$ yields $k=k_{\mathrm{c}}$ as defined in eq. (3.90). The phase transition point coincides with the spinodal $\chi_{\mathrm{c}}$, which in the symmetric case is not affected by compressibility. The fourth-order term, however, depends on $\lambda_{\text {eff }}$ and the number $Z$ of orientations. It has to be positive for all $Z$ to guarantee stability, thus we have to require that

$$
\lambda_{\text {eff }}>1
$$




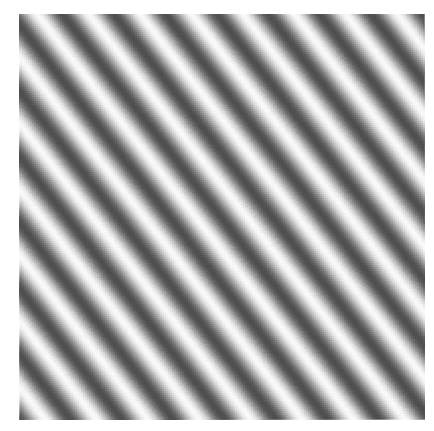

(a)

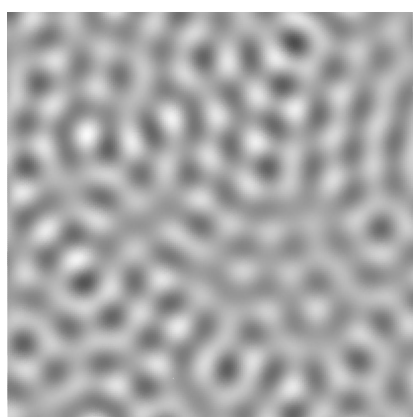

(d)

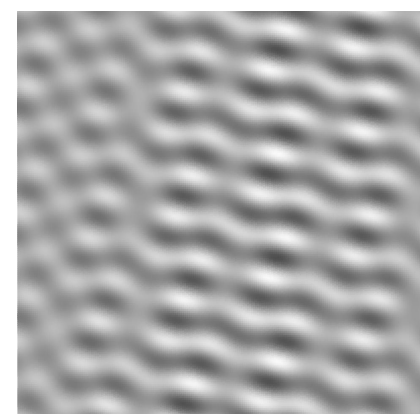

(b)

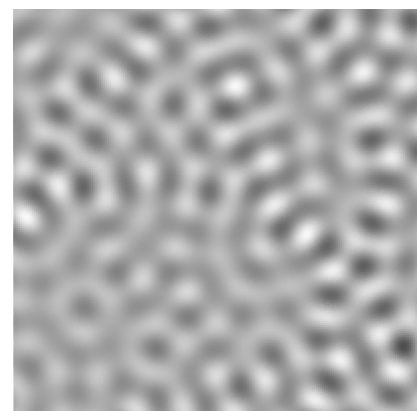

(e)

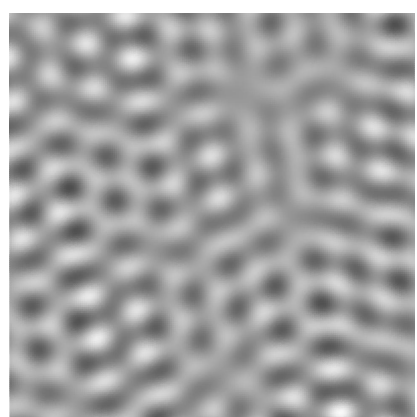

(c)

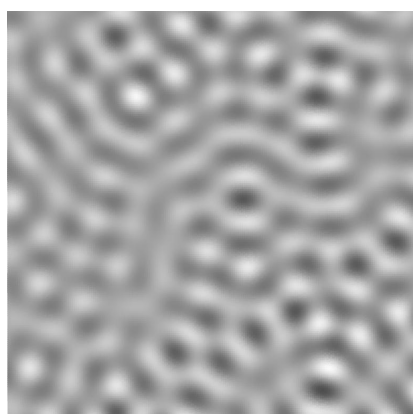

(f)

Figure 3.8.: Superposition of lamellæ raving random orientations in real space in two dimensions. The pictures show an area of 10 by 10 wavelengths, and the local amplitude is indicated by the grey scale in arbitrary units for $Z=1,5,10,50,100$ and 1000 orientations (from $(\mathrm{a})$ to $(\mathrm{f}))$.

The sign of the $\mathcal{O}\left(Z^{-1}\right)$ term determines the optimal number of random orientations. For low compressibilities, i.e. for $\lambda_{\text {eff }}>3 / 2$, the term is negative and the free energy grows with an increasing number of orientations, hence the simple lamellar morphology is favoured. For a rather compressible system having, in contrast, $1<\lambda_{\text {eff }}<3 / 2$, the effective free energy decreases with increasing $Z$, favouring an "infinite" number of orientations and hence a random pattern.

\subsection{Discussion and conclusions}

In this chapter we have studied various properties of weakly crosslinked homopolymer blends. Of particular interest were the enhancement of stability of the mixed regime, the scattering functions, i.e. the volatile and persistent concentration fluctuations, and the properties of the microphase separation transition replacing the macroscopic phase separation. Our investigations were performed on the basis of a Landau free energy derived from a comprehensive microscopic model. The latter accounts for both fundamental aspects in the system, viz. phase separation and gelation, following a modern vulcanisation theory [77] free of ad hoc assumptions, 
and treating quenched and annealed randomness systematically. Distinguishing between the preparation ensemble and the measurement ensemble, i.e. the system at the instant of crosslinking and at the time of measurement, respectively, our theory is able to quantify the degree to which demixing fluctuations in the blend are frozen-in by crosslinking.

In accordance with the de Gennes' prediction [45] and with virtually all other works on the subject, we have found the range of compatibility to be extended proportional to the degree $(\mu-1)$ of gelation and the occurrence of a peak in the scattering functions at a wavevector $k_{\mathrm{c}}$ (often denoted as $k^{*}$ ) growing linearly with $(\mu-1)$, as well. The latter approximately corresponds to the typical dimension of the strands between adjacent crosslinks or to the mesh size of the network. Some authors emphasise that they find larger domain sizes, i.e. a proportionality constant differing from de Gennes' by a factor of two or four; see, e.g., [49] or [56]. Lacking the exact relation between the localisation length of the gel and the typical strand length, we are unable to comment on this point. Yet, in the light of the rough estimate of the rigidity constant in [45] and of the high degree of disorder in the crosslinks implying a huge bandwidth of strand lengths, these deviations are rather small and not surprising. We have found, however, a substantial difference to [45] regarding the magnitude of the peak in the scattering functions, in agreement with Read et al. [53]: On approaching microphase separation, the peaks of $S(k)$ and $S_{\mathrm{gl}}(k)$ diverge as $\lambda_{2}^{-2} \propto\left(T-T_{\mathrm{c}}\right)^{-2}$ instead of $\left(T-T_{\mathrm{c}}\right)^{-1}$. The frozen-in disorder due to pre-crosslinking fluctuations and due to the random crosslinks seeds local rearrangements, leading to persistent concentration fluctuations that announce the onset of microphase separation. The purely thermal fluctuations grow as $\left(T-T_{\mathrm{c}}\right)^{-1}$, as predicted by de Gennes.

The most obvious deviation of the experiments from the theory in ref. [45] is the non-vanishing scattering intensity at zero scattering angle in the measurements. Briber and Bauer conjectured [49] that it originates from the frozen-in fluctuations, which was confirmed by several authors [51-53], however using phenomenological approaches with certain restrictions: Benmouna and Bettachy extended de Gennes' analogy to a dielectric by a Debye-Hückel screening term, which allows for (e.g. frozen-in) inhomogeneities on large length-scales. The screening length can be determined by assuming that the scattering functions of the uncrosslinked blend and the gel, measured at the preparation temperature, coincide [52]; as we have shown in section 3.5.6, this indeed holds true for the X-ray scattering function. This model does not explicitly account, though, for a fundamental property of the system: the localisation of polymer chains due to crosslinks. Read et al. [53] mimicked the immobilisation of the chains due to crosslinking by assuming that the chains are pinned at either end to randomly chosen points in space. As mentioned before, their (X-ray) scattering function diverges as $\left(T-T_{\mathrm{c}}\right)^{-2}$ and reveals nonzero intensity at zero wavenumber. Yet, the $a d$ hoc treatment only yields a caricature of the gel network, and it neither allows for the potential motion of crosslinks in the gel nor permits assessing the influence of the preparation conditions on the scattering intensities. 


\section{Crosslinked homopolymer blends}

Our present approach describes both phase separation and gelation in a microscopic manner, without resorting to ad hoc assumptions. Besides reproducing the peak in the scattering functions including its scaling near the microphase transition, and detecting the existence of the zero-angle scattering, we have also investigated the influence of the preparation conditions and the crossover between three limiting regimes, viz. preparation near demixing, measurement near demixing and weak gelation.

Furthermore, we have achieved a rough picture of the microphase separated state in the vicinity of the transition by assuming a regular or random morphology, taking into account the effects of compositional asymmetry and compressibility. As is to be expected from geometric considerations, lamellæ are advantageous in the symmetric case, while asymmetric melts favour a cubic lattice. Sufficiently compressible systems have been shown to prefer a random morphology. Due to the quenched randomness in the system, we expect only local ordering; according to Panyukov and Rubinstein [58], long-range order can, however, be restored by the application of stress, forcing local lamellæ to rotate into the direction of least modulus.

Although the microphase transition in the symmetric case is of second order within mean-field theory, it is likely to be rendered first-order by fluctuations, as was shown for generic systems with isotropic, finite- $k$ instabilities by Brazovskiu [44] and for block copolymer melts by Fredrickson and coworkers [5, 87]. The nonclassical region is, however, predicted to be small by Benhamou et al. [64]. 


\section{Conclusions and outlook}

We have studied the phase behaviour of two different kinds of molten heteropolymers with quenched randomness: random block copolymer melts and crosslinked homopolymer blends. In both systems, the frustration between the enthalpy of mixing and entropic forces hampers or prevents macroscopic phase separation, giving rise to microstructured phases with a variety of possible morphologies.

In chapter 2 we have addressed random block copolymers using different approaches derived from the same microscopic model. By coarse-graining the model, disregarding the conformations of the individual chains, we have recovered the multicomponent picture by Nesarikar et al. [16], which divides the copolymer chains into species according to their content of $A$ and $B$ monomers and determines the chemical equilibrium separately for each species. Due to the coarse-graining, the theory is restricted to separations into macroscopic phases, for which it is exact on the mean-field level also in the case of first order transitions. The analytic equilibrium conditions have been solved numerically. The alternative approach by Fredrickson et al. [17], based on a fourth-order Landau expansion of the effective free energy of the system, retains the microscopical details of the model and is thus capable of describing the separation into microscopic phases. We have extended the theory by Fredrickson et al. in different ways. We have shown that certain approximations for long blocks and chains in ref. [17] are inconsistent. For instance, they lead to the incorrect predictions that the system is insensitive to the microphase morphology and that the microphases reveal an infinite domain spacing at their onset in the symmetric case. According to our calculation, microphases in a symmetric melt always set in with finite wavelength and lamellar morphology. Abstaining from using said approximations, we have also been able to consider the case of short blocks and short chains, finding considerable deviations from the long-chain predictions. Furthermore, we have discussed the effects of compressibility, which faciliates phase separation and leads to a higher spinodal temperature in the asymmetric case. Comparing the predictions of the Landau theory and the multicomponent theory for macroscopic phase separation shows that the Landau expansion is only correct for nearly symmetric melts. For asymmetric melts, the transition becomes first-order and the Landau expansion breaks down.

Monte-Carlo simulations by Houdayer and Müller [21] suggest a fractionation of the melt according to the chain sequences, leading to the coexistence macro- and microphases, with homopolymeric chains preferring the former and copolymeric chains preferring the latter. In order to study the coexistence of homogeneous and microstructured phases, and, in particular, fractionation as a mechanism enabling microphase separation, we have set up a simple fractionation scheme. We have 


\section{Conclusions and outlook}

shown that fractionation is sufficient to enable microphase separation, without requiring the subtle relation between the spatial dependence of the second and fourth-order vertices of the Landau expansion necessary in the conventional theory by Fredrickson et al. [17]. Moreover, unlike ref. [22], our approach allows for the direct observation of the partitioning of the chains onto the three phases according to their sequence.

In chapter 3 we have developed a comprehensive model for randomly crosslinked homopolymer blends describing both gelation and phase separation in a microscopic fashion free of ad hoc assumptions. Moreover, our model accounts for different external conditions prior to and after preparation. In agreement with experiments [49] and previous phenomenological theories [45, 52, 53], we have found the stability of the mixed state to be enhanced by the introduction of crosslinks. We have computed the degree of stabilisation quantitatively for symmetric and asymmetric blends. Furthermore, our theory allowed us to calculate the scattering functions describing volatile and glassy, i.e. persistent, demixing fluctuations. Independently varying the conditions in the preparation and measurement ensembles and the amount of crosslinking, we have identified three regimes, in which the glassy fluctuations are dominated by either the pre-crosslinking fluctuations that are frozen-in into the gel network, by fluctuations seeded by frozen-in disorder, or by the fluctuations towards microphase separation. The latter are peaked at wavenumbers of about the inverse localisation length of the gel; they announce, and diverge at, the microphase transition.

The microphase separated state itself has been investigated considering different morphologies, including a random pattern. In incompressible symmetric systems, the lowest free energy is achieved by a lamellar lattice with a wavelength that corresponds to the localisation length of the gel and that only slightly varies with temperature on going deeper into the phase separated regime. Asymmetric compositions lead to a first-order transition, and the favoured microphase morphology are spherical domains of the minority component on a bcc lattice the matrix of the majority component in this case. Although mostly focusing on the incompressible limit, we have also studied the effects of compressibility, which partly contribute even in the incompressible limit. Moreover, we have shown that sufficiently compressible system prefer a random morphology to the regular microphase patterns.

\section{Further steps}

To generalise our theory of fractionation discussed in section 2.7 to the case of longer chains, it would be helpful to extend the class of polymer sequences allowed to "choose" between micro- and macrophases. Moreover, we have concentrated on fractionation of symmetric systems so far; it would be interesting to consider the asymmetric case, as well. Further points worth taking into account are the effects of fluctuations and polydispersity of blocks and chains. Since the enthalpy of mixing increases with the length of blocks and chains, whereas the entropy of mixing per chain is fixed, polydispersity has a major influence on the phase 
behaviour of real copolymer melts. It can lead to fractionation with respect to the chain length: the longest chains separate first to form microstructured phases, which coexist with the disordered phase of smaller chains [31].

In the discussion of crosslinked homopolymer blends in chapter 3, we concentrated, for the sake of simplicity, on the case of chains with equal degree of polymerisation before crosslinking. It would be interesting to disengage from this idealisation and to also study blends in which the two chain types have different lengths (still being monodisperse within the species); this should be technically straightforward. (The consideration of polydispersity would probably require major modifications of our theory.) A further possible extension is to consider a probability of crosslinking that depends on the monomer species involved. Such a model would cover, as limiting cases, interpenetrating and semi-interpenetrating networks, in which only chains of the same type or even chains of only one of the two types are crosslinked, respectively. It might also appear natural to go beyond mean-field theory, although, as pointed out before, mean-field theory is largely valid in the case of homopolymer blends [64].

Our technique appears well-suited to consider other crosslinked systems of interacting particles, as well. In our group, there are currently activities on nematic elastomers, with the long-term goal of developing a theory for spider silks [74]. 
4. Conclusions and outlook 


\section{Appendix}

\section{A. Mathematical tools}

\section{A. 1. Replica technique}

In the study of systems with quenched randomness, attention is paid mostly to the generic, i.e. average, system rather than to an instance with a particular realisation of disorder. Within the framework of statistical mechanics, this necessitates the computation of the mean free energy, thus basically the average logarithm of the partition function $Z$, which is often difficult. The replica trick, first proposed by Edwards and Anderson [81], reduces this task to the usually much easier calculation of the averages of integer powers of $Z$.

\section{A.1.1. Basic idea}

The replica method is based on two observations: First, the logarithm of $x$ can be expressed as a limit involving the $n$th power of $x$,

$$
\ln x=\lim _{n \rightarrow 0} \frac{x^{n}-1}{n} .
$$

Second, for natural $n \in \mathbb{N}$, the $n$th power of the partition function can be written as an explicit product. With the symbolic notation $Z=\int \mathcal{D} X \mathrm{e}^{-\beta H(X)}$, where $X$ runs over accessible states of the system, we obtain

$$
Z^{n}=\underbrace{Z \cdot Z \cdot \ldots \cdot Z}_{n \text { times }}=\int D^{n} X \mathrm{e}^{-\beta\left(H\left(X_{1}\right)+H\left(X_{2}\right)+\ldots+H\left(X_{n}\right)\right)} .
$$

Apparently, eq. (A.2) can be interpreted as the partition function of an $n$-fold replicated system with $n$ identical and independent (i.e. non-interacting) copies of the original system, the replicas. In replica calculations, adopting this interpretation, these $n$ copies are considered explicitly. The disorder averaged partition function $Z_{n}$ of the replicated system and the mean free energy per replica $F_{n}$ are defined through

$$
Z_{n}:=\exp \left(-\beta F_{n}\right):=\left[Z^{n}\right]
$$

where the disorder average is denoted by square brackets. After analytic continuation to non-integer $n$, eq. (A.1) can be used to relate $Z_{n}$ and $F_{n}$ to the desired mean free energy $F=-k_{\mathrm{B}} T[\ln Z]$ of the original, unreplicated system by

$$
F=-k_{\mathrm{B}} T \cdot \lim _{n \rightarrow 0} \frac{Z_{n}-1}{n}=\lim _{n \rightarrow 0} F_{n}
$$




\section{A.1.2. Replica symmetry}

Usually, the averaged replicated partition function $Z_{n}$ of a disordered system, defined in eq. (A.3), can only be computed approximately. A common method, also used in the present thesis, is to introduce a macroscopic order parameter $\phi$ and to express $Z_{n}$ as the integral of an effective function $\mathcal{Z}_{n}(\phi)$, with the microscopic states integrated out. On the mean-field level, $Z_{n}$ is approximated by maximum of $\mathcal{Z}_{n}$ over $\phi$; the corresponding value of $\phi$ can be interpreted as the macroscopic equilibrium state of the system.

The effective partition function is typically maximised using variational methods, making it necessary to guess a parametrisation of the order parameter and to decide whether it shall be replica-symmetric, i.e. invariant under permutations of the replicas, or if replica-symmetry breaking (RSB) is permitted. The latter allows a more proper description of systems with many degenerate ground-states and predicts a lower free energy than the replica-symmetric ansatz in such a case. However, replica-symmetry breaking schemes are rather intricate and an unnecessary complication for the purposes of the present thesis, so we don't further elaborate on RSB here. An introduction to replica symmetry breaking in the context of spin glasses is given in [88].

\section{A.2. Hubbard-Stratonovich transformations}

The Hubbard-Stratonovich transformations serve to linearise a quadratic exponent by means of Gaussian integration over an additional variable:

$$
\mathrm{e}^{+a\left(|w|^{2}-2 \operatorname{Re}\left(j w^{*}\right)\right)}=\frac{a}{\pi} \mathrm{e}^{-a|j|^{2}} \int \mathrm{d}(\operatorname{Re} z) \mathrm{d}(\operatorname{Im} z) \mathrm{e}^{-a\left(|z|^{2}-2 \operatorname{Re}\left(z w^{*}\right)+2 \operatorname{Re}\left(z j^{*}\right)\right)}
$$

and

$$
\mathrm{e}^{-a\left(|w|^{2}-2 \operatorname{Re}\left(j w^{*}\right)\right)}=\frac{a}{\pi} \mathrm{e}^{a|j|^{2}} \int \mathrm{d}(\operatorname{Re} z) \mathrm{d}(\operatorname{Im} z) \mathrm{e}^{-a\left(|z|^{2}-2 \operatorname{Re}\left(z w^{*}\right)+2 \operatorname{Re}\left(z j^{*}\right)\right)}
$$

for $a>0$ and $w, j, z \in \mathbb{C}$.

With these transformations we decouple the chains in the interactions and to reduce the many-chain problem to a single-chain problem. Furthermore, the auxiliary variables introduced in eqs. (A.5), (A.6) serve as macroscopic order parameters, in favour of which we can trace out the microscopic degrees of freedom to obtain a simpler, effective description of the system (see, e.g., section 3.4 .2 and appendix D). 


\section{B. Microphase morphologies}

To investigate microphase transition in both random copolymer melts and crosslinked homopolymer blends, we assume that the phase separation pattern can be described by a first-harmonic ansatz with a dominant wavenumber $k$ and a definite lattice structure:

$$
\Psi_{\boldsymbol{k}^{\prime}}=\Psi \frac{V}{\sqrt{2 m}} \sum_{i=1}^{m}\left(\delta_{\boldsymbol{k}^{\prime},+k \boldsymbol{n}_{i}}+\delta_{\boldsymbol{k}^{\prime},-k \boldsymbol{n}_{i}}\right),
$$

characterised by lattice vectors $n_{i} \in \mathbb{G}:=\left\{\boldsymbol{n}_{i} \mid i=1 \ldots m\right\}$ and an amplitude $\Psi$.

\section{B.1. Lattice structures}

Following Fredrickson et al. [17], we consider three particular morphologies, which are known to occur in the microphase separation of regular copolymer melts [3, 6]:

lamella $(m=1)$ : Alternating sheets rich in $A$ and $B$; one-dimensional order. Lattice vector: $\boldsymbol{n}_{1}=(1,0,0)^{T}$

cylinders $(m=3)$ : Close-packed, i.e. hexagonally arranged, cylindrical domains, $A$ in $B$ or vice versa; two-dimensional order. Lattice vectors: $\boldsymbol{n}_{1}=(1,0,0)^{T}$, $\boldsymbol{n}_{2}=(-1 / 2, \sqrt{3} / 2,0)^{T}$ and $\boldsymbol{n}_{3}=(-1 / 2,-\sqrt{3} / 2,0)^{T}$.

bcc spheres $(m=6)$ : Spherical $A$-rich domains in $B$, or vice versa, on a body centred cubic lattice in real space; three-dimensional order. Lattice vectors of the corresponding fcc lattice in Fourier space: $\boldsymbol{n}_{1}=(1,1,0)^{T} / \sqrt{2}, \boldsymbol{n}_{2}=$ $(0,1,1)^{T} / \sqrt{2}, \boldsymbol{n}_{3}=(1,0,1)^{T} / \sqrt{2}, \boldsymbol{n}_{4}=(1,0,-1)^{T} / \sqrt{2} \boldsymbol{n}_{5}=(-1,1,0)^{T} / \sqrt{2}$ and $\boldsymbol{n}_{6}=(0,-1,1)^{T} / \sqrt{2}$.

Note that $\boldsymbol{a} \in \mathbb{G} \Rightarrow-\boldsymbol{a} \notin \mathbb{G}$; therefore we introduce the symmetrised set of lattice vectors, $\mathbb{G}^{+}:=\{\boldsymbol{n} \mid \boldsymbol{n} \in \mathbb{G} \vee-\boldsymbol{n} \in \mathbb{G}\}$. For $m>1$, the set $\mathbb{G}$ of lattice vectors is not minimal in the sense of linear independence: for any two vectors $\boldsymbol{a} \neq \boldsymbol{b} \in \mathbb{G}$,

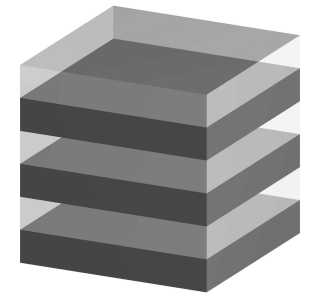

(a)

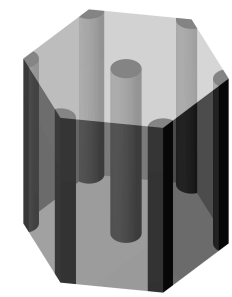

(b)

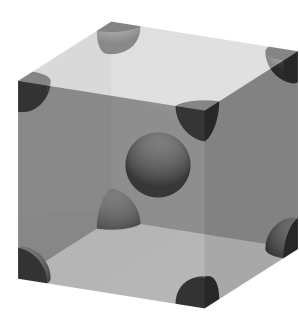

(c)

Figure B.1.: Regular lattices used in the ansatz for the morphology of the microphase separated state: lamellæ (a), hexagonally arranged cylinders $(\mathrm{b})$ and spherical domains on a bcc lattice (c). 
Appendix

\begin{tabular}{|c|c|c|c|c|}
\hline loop ty & & lamellæ & cylinders & bcc spheres \\
\hline 2-loop & & 2 & 6 & 12 \\
\hline 3-loop & & 0 & 12 & 48 \\
\hline 4-loop & planar & 6 & 90 & 396 \\
\hline & nonplanar & - & - & 144 \\
\hline & total & 6 & 90 & 540 \\
\hline
\end{tabular}

Table B.1.: Number of closed loops of $p$ lattice vectors (" $p$-loops") for different morphologies. The 4-loops are divided into planar and extra-planar loops.

the difference $\boldsymbol{a}-\boldsymbol{b}$ is included in $\mathbb{G}^{+}$. Rather, the vectors are rather chosen such that the $k_{i} \in \mathbb{G}^{+}$point towards the directions of all nearest-neighbour lattice sites.

For compressible homopolymer blends with crosslinks, we additionally consider a superposition of randomly oriented lamellæ, which is discussed in section 3.5.9.

Wavevector sums Inserting the ansatz (B.7) into the expansion of the Landau free energy of the random copolymer melt or the crosslinked homopolymer blend yields sums of the type

$$
\begin{aligned}
& \sum_{\boldsymbol{k}_{1, \ldots, p}}^{\prime} f_{2}\left(\boldsymbol{k}_{1}, \ldots, \boldsymbol{k}_{p-1}\right) \Psi_{\boldsymbol{k}_{1}} \ldots \Psi_{\boldsymbol{k}_{p}} \delta_{\sum_{\nu=1}^{p}} \boldsymbol{k}_{\nu}, \mathbf{0} \\
& =\frac{\Psi^{p} \cdot V^{p}}{(2 m)^{p / 2}} \sum_{\boldsymbol{k}_{1, \ldots, p}}^{\prime} f_{2}\left(\boldsymbol{k}_{1}, \ldots, \boldsymbol{k}_{p-1}\right) \prod_{\nu=1}^{p}\left(\sum_{\mu=1}^{m}\left(\delta_{\boldsymbol{k}_{\nu},+k \boldsymbol{n}_{\mu}}+\delta_{\boldsymbol{k}_{\nu},-k \boldsymbol{n}_{\mu}}\right)\right) \delta_{\sum_{\nu^{\prime}=1}^{p} \boldsymbol{k}_{\nu^{\prime}}, \mathbf{0}} \\
& =\Psi^{p} \cdot \frac{V^{p}}{(2 m)^{p / 2}} \sum_{\boldsymbol{k}_{1, \ldots, p} \in \mathbb{G}^{+}} f_{2}\left(\boldsymbol{k}_{1}, \ldots, \boldsymbol{k}_{p-1}\right) \delta_{\boldsymbol{k}_{1}+\ldots+\boldsymbol{k}_{p}, \mathbf{0}}
\end{aligned}
$$

In the quadratic sum the vertex function can be factored out, so that

$$
\frac{1}{V^{2}} \sum_{\boldsymbol{k}^{\prime}} f\left(k^{\prime 2}\right) \Psi_{\boldsymbol{k}^{\prime}} \Psi_{-\boldsymbol{k}^{\prime}}=\Psi^{2} \cdot \frac{f\left(k^{2}\right)}{2 m} \sum_{\boldsymbol{k}_{\mathbf{1}, \mathbf{2}} \in \mathbb{G}^{+}} \delta_{\boldsymbol{k}_{1}+\boldsymbol{k}_{2}, \mathbf{0}}=\Psi^{2} \cdot f\left(k^{2}\right) .
$$

The higher order sums depend strongly on the morphology of the microphases. In the simplest case $f\left(\boldsymbol{k}_{1}, \ldots\right)=1$, computing these loops amounts to counting the number of closed loops that can be constructed with the vectors in $\mathbb{G}^{+}$. In general, the loops must also be classified with respect to their shape, i.e. intra- or extra-planar, as distinct shapes yield distinct values of the vertex functions. The counting and classification have been done, e.g., in ref. [6], with the results shown in table B.1. For $f\left(\boldsymbol{k}_{1}, \ldots\right)=1$, the third- and fourth-order sums read

$$
\frac{1}{V^{3}} \sum_{\boldsymbol{k}_{1,2}} \Psi_{\boldsymbol{k}_{1}} \Psi_{\boldsymbol{k}_{2}} \Psi_{-\boldsymbol{k}_{1}-\boldsymbol{k}_{2}}=\Psi^{3} \cdot \begin{cases}0 & \text { for } m=1 \text { (lamellæ), } \\ \sqrt{2 / 3} & \text { for } m=3 \text { (cylinders), } \\ 2 / \sqrt{3} & \text { for } m=6 \text { (bcc spheres) }\end{cases}
$$


and

$$
\frac{1}{V^{4}} \sum_{\boldsymbol{k}_{1,2,3}} \Psi_{\boldsymbol{k}_{1}} \Psi_{\boldsymbol{k}_{2}} \Psi_{\boldsymbol{k}_{3}} \Psi_{-\boldsymbol{k}_{1}-\boldsymbol{k}_{2}-\boldsymbol{k}_{3}}=\Psi^{4} \cdot \begin{cases}3 / 2 & \text { for } m=1 \text { (lamellæ), } \\ 5 / 2 & \text { for } m=3 \text { (cylinders), } \\ 15 / 4 & \text { for } m=6 \text { (bcc spheres) }\end{cases}
$$




\section{Random copolymers: Auxiliary materials}

\section{C.1. Correlation of remote charges}

The disorder of chain sequences of random copolymers enters the Landau expansion of the according effective free energy: the vertex functions contain moments

$$
\left[q\left(s_{1}\right) \cdots q\left(s_{m}\right)\right]
$$

of monomer charges. In general, the moments correlate the monomers on different sites of a chain (charges on distinct chains are uncorrelated). In the following, we will compute these charge moments and use them to simplify the expressions for the vertex functions defined in section C.3. To compute these charge moments, we require the correlation between two charges on different sites of a chain. We define the probability vector

$$
\boldsymbol{p}(s):=\left(\begin{array}{c}
p_{A}(s) \\
p_{B}(s)
\end{array}\right)=\left(\begin{array}{c}
p_{A}(s) \\
1-p_{A}(s)
\end{array}\right)
$$

indicating the probabilities that monomer $s$ is of type $A$ or $B$. For monomers $s_{1,2}$ on the same block, obviously $\boldsymbol{p}\left(s_{1}\right)=\boldsymbol{p}\left(s_{2}\right)$; the a priori probability vector reads $\boldsymbol{p}(s)=(p, 1-p)^{T}$. From the definition of the disorder in section 2.2 .2 we know that the charges on neighbouring blocks depend via

$$
\boldsymbol{p}(s \pm M)=\mathbf{M} \boldsymbol{p}(s)
$$

with the transition matrix (2.6). We diagonalise $\mathbf{M}$,

$$
\tilde{\mathbf{M}}=\mathbf{D ~ M ~ D ~}^{-1}=\left(\begin{array}{ll}
\lambda & 0 \\
0 & 1
\end{array}\right) \quad \text { with } \quad \mathbf{D}=\left(\begin{array}{cc}
1-p & -p \\
1 & 1
\end{array}\right)
$$

and compute

$$
\begin{aligned}
\mathbf{M}^{\Delta M} & =\mathbf{D}^{-1} \tilde{\mathbf{M}} \mathbf{D}=\mathbf{D}^{-1}\left(\begin{array}{cc}
\lambda^{\Delta M} & 0 \\
0 & 1
\end{array}\right) \mathbf{D} \\
& =\left(\begin{array}{cc}
1-(1-p)\left(1-\lambda^{\Delta M}\right) & p\left(1-\lambda^{\Delta M}\right) \\
(1-p)\left(1-\lambda^{\Delta M}\right) & 1-p\left(1-\lambda^{\Delta M}\right)
\end{array}\right)=:\left(\begin{array}{ll}
\mathrm{M}_{A A}^{\Delta M} & \mathrm{M}_{B A}^{\Delta M} \\
\mathrm{M}_{A B}^{\Delta M} & \mathrm{M}_{B B}^{\Delta M}
\end{array}\right),
\end{aligned}
$$

so that we can generalise eq. (C.14) to

$$
\boldsymbol{p}\left(s_{1}\right)=\left(\begin{array}{ll}
\mathrm{M}_{A A}^{\Delta M} & \mathrm{M}_{B A}^{\Delta M} \\
\mathrm{M}_{A B}^{\Delta M} & \mathrm{M}_{B B}^{\Delta M}
\end{array}\right) \boldsymbol{p}\left(s_{2}\right),
$$

with two monomers $s_{1,2}$ located $\Delta M$ blocks apart (regardless of the positions within the blocks). 


\section{C.1.1. Charge moments}

As the charges are block-wise equal, it is convenient to introduce the block number

$$
\sigma(s):=\min _{z \in \mathbb{Z}}\{z \geq s / M\} \quad \in\{1,2, \ldots, Q\}
$$

The expectation value of a single charge is given by $[q(s)]=2 p-1=q$, see eq. (2.12); the second moment is computed as follows:

$$
\begin{aligned}
{\left[q\left(s_{1}\right) q\left(s_{2}\right)\right]=} & \mathbb{P}(q(s 2)=q(s 1)=+1)-\mathbb{P}(q(s 2)=-q(s 1)=+1) \\
& \mathbb{P}(q(s 2)=q(s 1)=-1)-\mathbb{P}(q(s 2)=-q(s 1)=-1) \\
= & p \cdot\left(\mathrm{M}_{A A}^{\Delta M}-\mathrm{M}_{A B}^{\Delta M}\right)+(1-p) \cdot\left(\mathrm{M}_{B B}^{\Delta M}-\mathrm{M}_{B A}^{\Delta M}\right) \\
= & 1-\left(1-q^{2}\right)\left(1-\lambda^{\left|\sigma\left(s_{2}\right)-\sigma\left(s_{1}\right)\right|}\right),
\end{aligned}
$$

$N B$ including the case $\sigma\left(s_{1}\right)=\sigma\left(s_{2}\right)$. The higher moments can be calculated analogously.

\section{C.1.2. Centred charges}

In section 2.5.2 the density field is manipulated to make the vertices of the free energy expansion contain the moments of the centred charges $\check{q}(s)=q(s)-q$, because the centred version yields simpler expressions than the original ones. We obtain

$$
\begin{array}{lll}
{\left[\check{q}\left(s_{1}\right)\right]} & =0 \\
{\left[\check{q}\left(s_{1}\right) \check{q}\left(s_{2}\right)\right]} & =\left(1-q^{2}\right) \lambda^{\left|\sigma\left(s_{2}\right)-\sigma\left(s_{1}\right)\right|} \\
{\left[\check{q}\left(s_{1}\right) \check{q}\left(s_{2}\right) \check{q}\left(s_{3}\right)\right]} & =-2 q\left(1-q^{2}\right) \lambda^{\max \left\{\sigma\left(s_{1,2,3}\right)\right\}-\min \left\{\sigma\left(s_{1,2,3}\right)\right\}}
\end{array}
$$

for $s_{\nu}$ in arbitrary order and

$$
\begin{aligned}
{\left[\check{q}\left(s_{1}\right) \cdots \check{q}\left(s_{4}\right)\right]=} & \left(1-q^{2}\right) \lambda^{\sigma\left(s_{4}\right)-\sigma\left(s_{1}\right)}\left(4 q^{2}+\left(1-q^{2}\right) \lambda^{\sigma\left(s_{2}\right)-\sigma\left(s_{3}\right)}\right) \\
{\left[\check{q}\left(s_{1}\right) \cdots \check{q}\left(s_{5}\right)\right]=- } & 2 q\left(1-q^{2}\right) \lambda^{\sigma\left(s_{5}\right)-\sigma\left(s_{1}\right)} \\
& \times\left(4 q^{2}+\left(1-q^{2}\right)\left(\lambda^{\sigma\left(s_{3}\right)-\sigma\left(s_{4}\right)}+\lambda^{\sigma\left(s_{2}\right)-\sigma\left(s_{3}\right)}\right)\right) \\
{\left[\check{q}\left(s_{1}\right) \cdots \check{q}\left(s_{6}\right)\right]=} & \left(1-q^{2}\right) \lambda^{\sigma\left(s_{6}\right)-\sigma\left(s_{1}\right)} \times \\
& \left(4 q^{2}\left(1-q^{2}\right)\left(\lambda^{\sigma\left(s_{4}\right)-\sigma\left(s_{5}\right)}+\lambda^{\sigma\left(s_{3}\right)-\sigma\left(s_{4}\right)}+\lambda^{\sigma\left(s_{2}\right)-\sigma\left(s_{3}\right)}\right)\right. \\
& \left.+(1-q)^{2} \lambda^{\sigma\left(s_{4}\right)-\sigma\left(s_{5}\right)+\sigma\left(s_{2}\right)-\sigma\left(s_{3}\right)}+16 q^{4}\right)
\end{aligned}
$$

for $\left\{s_{\nu}\right\}$ with block numbers $\sigma\left(s_{1}\right) \leq \sigma\left(s_{2}\right) \leq \cdots \leq \sigma\left(s_{6}\right)$ in ascending order. 


\section{C.2. Wiener correlator}

The vertex functions appearing in the Landau expansion of the effective free energy (2.69) are essentially sums over the replicated version of Wiener correlators of the type

$$
\begin{aligned}
\left\langle\exp \left\{-\mathrm{i} \sum_{\zeta=1}^{z} \boldsymbol{k}_{\zeta} \boldsymbol{r}\left(s_{\zeta}\right)\right\}\right\rangle & =\delta_{\sum_{\zeta=1}^{z} \boldsymbol{k}_{\zeta}, \mathbf{0}} \exp \left\{-\sum_{\zeta, \zeta^{\prime}} \min \left\{s_{\zeta}-s_{\zeta^{\prime}}\right\} \boldsymbol{k}_{\zeta} \boldsymbol{k}_{\zeta^{\prime}}\right\} \\
& =\delta_{\sum_{\zeta=1}^{z} \boldsymbol{k}_{\zeta}, \mathbf{0}} \exp \left\{\frac{1}{2} \sum_{\zeta, \zeta^{\prime}}\left|s_{\zeta}-s_{\zeta^{\prime}}\right| \boldsymbol{k}_{\zeta} \boldsymbol{k}_{\zeta^{\prime}}\right\},
\end{aligned}
$$

averaged with respect to the Wiener Hamiltonian (2.17). (A derivation of the continuous chain analogue of eq. (C.27) can be found in [77]; the transfer to discrete chains is straightforward.) The replicated correlator factorises in the replica index and vanishes unless the wavevectors sum to zero within each replica,

$$
\begin{aligned}
\left\langle\exp \left\{-\mathrm{i} \sum_{\zeta_{1}=1}^{z_{1}} \boldsymbol{k}_{\zeta_{1}} \cdot \boldsymbol{r}^{\alpha_{1}}\left(s_{\zeta_{1}}\right)-\ldots-\mathrm{i} \sum_{\zeta_{m}=1}^{z_{m}} \boldsymbol{k}_{\zeta_{m}} \cdot \boldsymbol{r}^{\alpha_{m}}\left(s_{\zeta_{m}}\right)\right\}\right\rangle= \\
\left\langle\exp \left\{-\mathrm{i} \sum_{\zeta_{1}=1}^{z_{1}} \boldsymbol{k}_{\zeta_{1}} \boldsymbol{r}\left(s_{\zeta_{1}}\right)\right\}\right\rangle \ldots\left\langle\exp \left\{-\mathrm{i} \sum_{\zeta_{m}=1}^{z_{m}} \boldsymbol{k}_{\zeta_{m}} \boldsymbol{r}\left(s_{\zeta_{m}}\right)\right\}\right\rangle
\end{aligned}
$$

for pairwise distinct $\alpha_{1}, \ldots, \alpha_{m}$, where $\langle\cdots\rangle$ in the first line of eq. (C.28) denotes the replicated Wiener average (2.62).

\section{C.3. Vertex functions}

In this section we define and partially compute the correlation vertex functions occurring in the Landau expansion of the effective free energy (2.69).

\section{C.3.1. Discrete Debye function}

The simplest vertex function, containing no charge moments, reads

$$
\begin{aligned}
D\left(k^{2}\right) & :=\sum_{s_{1,2}=1}^{L}\left\langle\mathrm{e}^{-\mathrm{i} \boldsymbol{k}\left(\boldsymbol{r}\left(s_{1}\right)-\boldsymbol{r}\left(s_{2}\right)\right)}\right\rangle=\sum_{s_{1,2}=1}^{L} \mathrm{e}^{-k^{2}\left|s_{2}-s_{1}\right|} \\
& =\frac{1+\mathrm{e}^{-k^{2}}}{1-\mathrm{e}^{-k^{2}}} \cdot L-\frac{2 \mathrm{e}^{-k^{2}}\left(1-\mathrm{e}^{-k^{2} L}\right)}{\left(1-\mathrm{e}^{-k^{2}}\right)^{2}}
\end{aligned}
$$

its characteristic scale is the inverse square radius of gyration of the chains, $1 / R_{L}^{2}=$ $1 / L$. For $L k^{2} \ll 1$, we can expand

$$
D\left(k^{2}\right)=L^{2}-\frac{L^{2}-1}{3} \cdot\left(L k^{2}\right)+\frac{L^{2}-1}{12} \cdot\left(L k^{2}\right)^{2}+\mathcal{O}\left(L^{2} \cdot\left(L k^{2}\right)^{3}\right) .
$$


For long chains it is justified to approximate $D\left(k^{2}\right)$ by its continuum limit. To that end, the number $L$ of monomers and the effective bond length $b$ (see eq. (2.2)) are rescaled as $L \rightarrow L \cdot f$ and $b \rightarrow b / \sqrt{f}$, so that the radius of gyration of the chain is conserved. In section 2.2.3, the units were chosen such that initially $b=1$; effectively, the scaling of $b \rightarrow b / \sqrt{f}$ leads to rescaled $k^{2} \rightarrow k^{2} / f$. The continuum limit $f \rightarrow \infty$ yields the Debye function defined in appendix D.2.1,

$$
\left.\lim _{L \rightarrow \infty} \frac{D\left(k^{2}\right)}{L^{2}}\right|_{b^{2}=1 / L}=g_{\mathrm{D}}\left(L k^{2}\right)=\frac{\mathrm{e}^{-L k^{2}}-1+L k^{2}}{\left(L k^{2}\right)^{2} / 2} .
$$

We also define the single-block analogue of $D\left(k^{2}\right)$,

$$
D^{(M)}\left(k^{2}\right):=\sum_{t_{1,2}=1}^{M} \mathrm{e}^{-k^{2}\left|t_{2}-t_{1}\right|}=\frac{1+\mathrm{e}^{-k^{2}}}{1-\mathrm{e}^{-k^{2}}} \cdot M-\frac{2 \mathrm{e}^{-k^{2}}\left(1-\mathrm{e}^{-k^{2} M}\right)}{\left(1-\mathrm{e}^{-k^{2}}\right)^{2}} .
$$

\section{C.3.2. Second-order charge correlator}

The quadratic vertex of the charge density field contains the correlation function

$$
\begin{aligned}
& S\left(k^{2}\right):=\sum_{s_{1,2}=1}^{L} \frac{\left[\check{q}\left(s_{1}\right) \check{q}\left(s_{2}\right)\right]}{1-q^{2}}\left\langle\mathrm{e}^{-\mathrm{i} \boldsymbol{k}\left(\boldsymbol{r}\left(s_{1}\right)-\boldsymbol{r}_{2}\left(s_{2}\right)\right)}\right\rangle=\sum_{s_{1,2}=1}^{M Q} \lambda^{\left|\sigma\left(s_{2}\right)-\sigma\left(s_{1}\right)\right|} \cdot \mathrm{e}^{-k^{2}\left|s_{2}-s_{1}\right|} \\
& =Q \cdot D^{(M)}\left(k^{2}\right)+\frac{\sinh ^{2}\left(M k^{2} / 2\right)}{\sinh ^{2}\left(k^{2} / 2\right)} \cdot \frac{2 \lambda \mathrm{e}^{-M k^{2}}}{1-\lambda \mathrm{e}^{-M k^{2}}}\left(Q-\frac{1-\left(\lambda \mathrm{e}^{-M k^{2}}\right)^{Q}}{1-\lambda \mathrm{e}^{-M k^{2}}}\right),
\end{aligned}
$$

the long wavelength limit of which reads

$$
\lim _{k \rightarrow 0} \frac{S\left(k^{2}\right)}{M^{2} Q}=\frac{1+\lambda}{1-\lambda}-\frac{2 \lambda}{Q} \cdot \frac{1-\lambda^{Q}}{(1-\lambda)^{2}} .
$$

Eq. (C.34) can be expanded in powers of $\lambda$ yielding

$$
S\left(k^{2}\right)=Q \cdot D^{(M)}\left(k^{2}\right)+2(Q-1) \lambda \mathrm{e}^{-k^{2}}\left(\frac{1-\mathrm{e}^{-M k^{2}}}{1-\mathrm{e}^{-k^{2}}}\right)^{2}+\mathcal{O}\left(\lambda^{2}\right) .
$$

Continuum limits The function $S\left(k^{2}\right)$ depends on the number $Q$ and the size $M$ of the blocks, and we can take the continuum limit in both. For the limit of continuous blocks, the effective bond length $b$ has to be rescaled like in the continuum limit of $D\left(k^{2}\right)$ to preserve the radius of gyration, and we obtain

$$
\begin{aligned}
\left.\lim _{M \rightarrow \infty} \frac{S\left(k^{2}\right)}{M^{2} Q}\right|_{b^{2}=1 / M} & = \\
& g_{\mathrm{D}}\left(M k^{2}\right)+\frac{\sinh ^{2}\left(M k^{2} / 2\right)}{\left(M k^{2}\right)^{2}} \cdot \frac{8 \lambda \mathrm{e}^{-M k^{2}}}{1-\lambda \mathrm{e}^{-M k^{2}}}\left(1-\frac{1-\left(\lambda \mathrm{e}^{-M k^{2}}\right)^{Q}}{Q\left(1-\lambda \mathrm{e}^{-M k^{2}}\right)}\right) .
\end{aligned}
$$


For a large number $Q$ of discrete blocks, where $\lambda^{Q}$ becomes small unless $\lambda= \pm 1$, we have

$$
\lim _{Q \rightarrow \infty} \frac{S\left(k^{2}\right)}{Q}=D^{(M)}\left(k^{2}\right)+\frac{\sinh ^{2}\left(M k^{2} / 2\right)}{\sinh ^{2}\left(k^{2} / 2\right)} \cdot \frac{2 \lambda \mathrm{e}^{-M k^{2}}}{1-\lambda \mathrm{e}^{-M k^{2}}} .
$$

Taking both limits (in arbitrary order) yields

$$
s\left(M k^{2}\right):=\left.\lim _{M, Q \rightarrow \infty} \frac{S\left(k^{2}\right)}{Q M^{2}}\right|_{b^{2}=1 / M}=g_{\mathrm{D}}\left(M k^{2}\right)+\frac{\sinh ^{2}\left(M k^{2} / 2\right)}{\left(M k^{2}\right)^{2}} \cdot \frac{8 \lambda \mathrm{e}^{-M k^{2}}}{1-\lambda \mathrm{e}^{-M k^{2}}},
$$

which corresponds to $4 \cdot G_{2}\left(M k^{2}\right) / M\left(1-q^{2}\right)$ of reference [17].

\section{C.3.3. Higher-order correlators}

The charge moments introduce a block-wise ordering of the monomer index (see section C.1.2). To compute the sums in the vertex functions they must therefore be split into partial sums with definite order. The computational effort for this chiefly combinatorial problem grows exponentially with the degree of the vertex. Hence we shall abstain from carrying out the sums in the higher-order correlators defined in the following, aside from an approximation for long and nearly uncorrelated chains in section C.3.4.

Charge-charge-mass correlation The vertex function of the $\Psi^{2} \Omega$ term in the free energy expansion is given by

$$
\begin{aligned}
& S_{2}\left(\boldsymbol{k}_{1}, \boldsymbol{k}_{2}\right):=\sum_{s_{1,2,3}=1}^{L}\left.\frac{\left[\check{q}\left(s_{1}\right) \check{q}\left(s_{2}\right)\right]}{1-q^{2}}\left\langle\mathrm{e}^{-\mathrm{i} \sum_{\nu=1}^{3} \boldsymbol{k}_{\nu} \boldsymbol{r}\left(s_{\nu}\right)}\right\rangle\right|_{\boldsymbol{k}_{3}=-\boldsymbol{k}_{1}-\boldsymbol{k}_{2}} \\
&=\left.\sum_{s_{1,2,3}=1}^{L} \lambda^{\left|\sigma\left(s_{2}\right)-\sigma\left(s_{1}\right)\right|} \mathrm{e}^{-\sum_{\nu, \nu^{\prime}} \min \left\{s_{\nu}, s_{\nu^{\prime}}\right\} \boldsymbol{k}_{\nu} \boldsymbol{k}_{\nu^{\prime}}}\right|_{\boldsymbol{k}_{3}=-\boldsymbol{k}_{1}-\boldsymbol{k}_{2}},
\end{aligned}
$$

which for opposite $\boldsymbol{k}_{1,2}$ reduces to the quadratic charge correlator,

$$
S_{2}(\boldsymbol{k},-\boldsymbol{k})=L \cdot S\left(k^{2}\right) .
$$

From eq. (C.40) it follows that

$$
S_{2}(\mathbf{0}, \mathbf{0})=M^{3} Q^{2}\left(\frac{1+\lambda}{1-\lambda}-\frac{2 \lambda}{Q} \cdot \frac{1-\lambda^{Q}}{(1-\lambda)^{2}}\right) .
$$

Charge-charge-charge correlation For the $\Psi^{3}$ term we require

$$
\begin{aligned}
S_{3}\left(\boldsymbol{k}_{1}, \boldsymbol{k}_{2}\right):=\left.\sum_{s_{1,2,3}} \frac{\left[\check{q}\left(s_{1}\right) \check{q}\left(s_{2}\right) \check{q}\left(s_{3}\right)\right]}{2 q\left(1-q^{2}\right)}\left\langle\mathrm{e}^{-\mathrm{i} \sum_{\nu=1}^{3} \boldsymbol{k}_{\nu} \boldsymbol{r}\left(s_{\nu}\right)}\right\rangle\right|_{\boldsymbol{k}_{3}=-\boldsymbol{k}_{1}-\boldsymbol{k}_{2}} \\
\quad=\left.\sum_{s_{1,2,3}=1}^{L} \lambda^{\max \left\{\sigma\left(s_{1,2,3}\right)\right\}-\min \left\{\sigma\left(s_{1,2,3}\right)\right\}} \mathrm{e}^{-\sum_{\nu, \nu^{\prime}} \min \left\{s_{\nu}, s_{\nu^{\prime}}\right\} \boldsymbol{k}_{\nu} \boldsymbol{k}_{\nu^{\prime}}}\right|_{\boldsymbol{k}_{3}=-\boldsymbol{k}_{1}-\boldsymbol{k}_{2}} .
\end{aligned}
$$


Fourth-order charge correlations There are two different fourth-order vertex functions, originating from a single-replica and a two-replica term, respectively:

$$
\begin{aligned}
S_{4}\left(\boldsymbol{k}_{1}, \boldsymbol{k}_{2}, \boldsymbol{k}_{3}\right):=\left.\sum_{s_{1,2,3,4}=1}^{L}\left[\check{q}\left(s_{1}\right) \check{q}\left(s_{2}\right) \check{q}\left(s_{3}\right) \check{q}\left(s_{4}\right)\right]\left\langle\mathrm{e}^{-\mathrm{i} \sum_{\nu=1}^{4} \boldsymbol{k}_{\nu} \boldsymbol{r}\left(s_{\nu}\right)}\right\rangle\right|_{\boldsymbol{k}_{4}=-\sum_{\nu=1}^{3} \boldsymbol{k}_{\nu}} \\
=\left(1-q^{2}\right) \sum_{s_{1,2,3,4}} \lambda^{\sigma_{4}^{\prime}-\sigma_{1}^{\prime}}\left(4 q^{2}+\left(1-q^{2}\right) \lambda^{\sigma_{2}^{\prime}-\sigma_{3}^{\prime}}\right) \\
\times\left.\mathrm{e}^{-\sum_{\nu, \nu^{\prime}} \min \left\{s_{\nu}, s_{\nu^{\prime}}\right\} \boldsymbol{k}_{\nu} \boldsymbol{k}_{\nu^{\prime}}}\right|_{\boldsymbol{k}_{4}=-\sum_{\nu=1}^{3} \boldsymbol{k}_{\nu}}
\end{aligned}
$$

and

$$
\begin{aligned}
S_{5}\left(\boldsymbol{k}, \boldsymbol{k}^{\prime}\right) & :=\sum_{s_{1,2,3,4}}\left[\check{q}\left(s_{1}\right) \check{q}\left(s_{2}\right) \check{q}\left(s_{3}\right) \check{q}\left(s_{4}\right)\right]\left\langle\mathrm{e}^{-\mathrm{i} \boldsymbol{k}\left(\boldsymbol{r}\left(s_{1}\right)-\boldsymbol{r}\left(s_{2}\right)\right)}\right\rangle\left\langle\mathrm{e}^{-\mathrm{i} \boldsymbol{k}^{\prime}\left(\boldsymbol{r}\left(s_{3}\right)-\boldsymbol{r}\left(s_{4}\right)\right)}\right\rangle \\
& =\left(1-q^{2}\right) \sum_{s_{1,2,3,4}} \lambda^{\sigma_{4}^{\prime}-\sigma_{1}^{\prime}}\left(4 q^{2}+\left(1-q^{2}\right) \lambda^{\sigma_{2}^{\prime}-\sigma_{3}^{\prime}}\right) \mathrm{e}^{-k^{2}\left|s_{2}-s_{1}\right|-k^{\prime 2}\left|s_{4}-s_{3}\right|}
\end{aligned}
$$

both defined with the renumbered block indices $\left\{\sigma_{\nu}^{\prime}\right\}:=\left\{\sigma\left(s_{\nu}\right)\right\}$ sorted in ascending order $\sigma_{1}^{\prime} \leq \sigma_{2}^{\prime} \leq \sigma_{3}^{\prime} \leq \sigma_{4}^{\prime}$.

\section{C.3.4. Long chain approximation}

In the following approximations, the sums in the higher-order vertex function can be expressed in terms of the discrete Debye function $D\left(k^{2}\right)$ of (C.30): Assuming small sequence correlations $\lambda \ll 1$, the correlators are expanded just up to first order in $\lambda$. Furthermore, we let $k^{2}\left(s_{1}-s_{2}\right) \approx 0$ for $\left|\sigma\left(s_{1}\right)-\sigma\left(s_{2}\right)\right| \leq 1$, neglecting the decay of correlations of monomers located on the same block or on neighbouring ones; this is justified as a coarse-graining in the case of chains of many small blocks. These approximations are suggested in [17], together with the neglect of subdominant terms in the number of blocks $Q$, consistent with the coarse-graining.

With coarse-grained blocks, eq. (C.35) becomes

$$
S\left(k^{2}\right) \approx(1+2 \lambda) Q M^{2}-2 \lambda M^{2}+\mathcal{O}\left(\lambda^{2}\right) .
$$

For the third-order correlators, the above approximations lead to

$$
S_{2}\left(\boldsymbol{k}_{1}, \boldsymbol{k}_{2}\right) \approx(1+2 \lambda) M D\left(\left(\boldsymbol{k}_{1}+\boldsymbol{k}_{2}\right)^{2}\right)-2 \lambda M^{3} Q+\mathcal{O}\left(\lambda^{2}\right)
$$

and

$$
S_{3}\left(\boldsymbol{k}_{1}, \boldsymbol{k}_{2}\right) \approx(1+6 \lambda) M^{3} Q-6 M^{3} \lambda \quad+\mathcal{O}\left(\lambda^{2}\right)
$$

the fourth-order correlators reduce to

$$
\begin{aligned}
& S_{4}\left(\boldsymbol{k}_{1}, \boldsymbol{k}_{2}, \boldsymbol{k}_{3}\right) \approx 4 q^{2}\left(1-q^{2}\right)\left\{(1+14 \lambda) M^{4} Q-14 \lambda M^{4}\right\} \\
& +\left(1-q^{2}\right)^{2}\left\{(1+4 \lambda) M^{2}\left(D\left(\left(\boldsymbol{k}_{1}+\boldsymbol{k}_{2}\right)^{2}\right)+D\left(\left(\boldsymbol{k}_{2}+\boldsymbol{k}_{3}\right)^{2}\right)+D\left(\left(\boldsymbol{k}_{3}+\boldsymbol{k}_{1}\right)^{2}\right)\right)\right. \\
& \left.-(1+14 \lambda) \cdot 2 M^{4} Q+16 \lambda M^{4}\right\}+\mathcal{O}\left(\lambda^{2}\right) \quad(\mathrm{C} .
\end{aligned}
$$


Appendix

and

$$
\begin{aligned}
& S_{5}\left(\boldsymbol{k}, \boldsymbol{k}^{\prime}\right) \approx 4 q^{2}\left(1-q^{2}\right)\left\{(1+14 \lambda) M^{4} Q-14 \lambda M^{4}\right\} \\
& +\left(1-q^{2}\right)^{2}\left\{(1+4 \lambda) M^{2}\left(M^{2} Q^{2}+2 D\left(k^{2}+k^{2}\right)\right)\right. \\
& \left.\quad-(1+14 \lambda) \cdot 2 M^{4} Q+16 \lambda M^{4}\right\}+\mathcal{O}\left(\lambda^{2}\right) .
\end{aligned}
$$

\section{C.4. Landau free energy of compressible melts}

The fourth-order Landau expansion of the effective free energy (2.69), with the shifted density field $\Omega$ traced out up to quadratic order in $\Omega$, reads

$$
\begin{aligned}
\mathcal{F}_{n}(\bar{\Omega}, \Psi) \approx & \frac{N}{8 V^{2}} \sum_{\alpha} \sum_{\boldsymbol{k}}^{\prime}\left(\frac{2 V}{N \chi W_{k}}-\left(1-q^{2}\right) S\left(k^{2}\right)-\frac{q^{2} D\left(k^{2}\right)}{1+N \mu U_{k} D\left(k^{2}\right) / 2 V}\right) \Psi_{\boldsymbol{k}}^{\alpha} \Psi_{-\boldsymbol{k}}^{\alpha} \\
+ & \frac{N q\left(1-q^{2}\right)}{24 V^{3}} \sum_{\alpha} \sum_{\boldsymbol{k}_{1,2}}^{\prime}\left(S_{3}\left(\boldsymbol{k}_{1}, \boldsymbol{k}_{2}\right)-\frac{3 / 2 \cdot S_{2}\left(\boldsymbol{k}_{1}, \boldsymbol{k}_{2}\right)}{1+N \mu U_{k_{3}} D\left(k_{3}^{2}\right) / 2 V}\right) \\
+ & \frac{N}{128 V^{4}} \sum_{\alpha} \sum_{\boldsymbol{k}_{1,2,3,4}}^{\prime}\left(\left(1-q^{2}\right)^{2} \cdot \frac{S_{\boldsymbol{k}_{1}+\boldsymbol{k}_{2}+\boldsymbol{k}_{3}, \mathbf{0}} \Psi_{\boldsymbol{k}_{1}}^{\alpha} \Psi_{\boldsymbol{k}_{2}}^{\alpha} \Psi_{\boldsymbol{k}_{3}}^{\alpha}}{D\left(\left(\boldsymbol{k}_{1}, \boldsymbol{k}_{2}\right) S_{2}\left(\boldsymbol{k}_{3}, \boldsymbol{k}_{4}\right)\right.}\right. \\
& \left.-\frac{\left.N\left(1-\boldsymbol{k}_{2}\right)^{2}\right)+2 V /\left(N \mu U_{\boldsymbol{k}_{1}+\boldsymbol{k}_{2}}\right)}{128 V^{4}} \frac{1}{1+2 V /\left(N L^{2} \mu U_{0}\right)} \sum_{\alpha}\left(\sum_{\boldsymbol{k}}^{\prime} S_{4}\left(\boldsymbol{k}_{1}, \boldsymbol{k}_{2}, \boldsymbol{k}_{3}\right)\right) \delta_{\sum_{\nu=1}^{4} \boldsymbol{k}_{\nu}, \mathbf{0}} \Psi_{\boldsymbol{k}_{1}}^{\alpha} \Psi_{\boldsymbol{k}_{2}}^{\alpha} \Psi_{\boldsymbol{k}_{3}}^{\alpha} \Psi_{\boldsymbol{k}_{4}}^{\alpha} \Psi_{-\boldsymbol{k}}^{\alpha}\right)^{2} \\
+ & \frac{N\left(1-q^{2}\right)^{2}}{128 V^{4}} \sum_{\alpha_{1,2}} \sum_{\boldsymbol{k}_{1,2}}^{\prime} S\left(k_{1}^{2}\right) S\left(k_{2}^{2}\right) \Psi_{\boldsymbol{k}_{1}}^{\alpha_{1}} \Psi_{-\boldsymbol{k}_{1}}^{\alpha_{1}} \Psi_{\boldsymbol{k}_{2}}^{\alpha_{2}} \Psi_{-\boldsymbol{k}_{2}}^{\alpha_{2}} \\
- & \frac{N}{128 V^{4}} \sum_{\alpha 1 \neq \alpha 2} \sum_{\boldsymbol{k}_{1,2}} S_{5}\left(\boldsymbol{k}_{1}, \boldsymbol{k}_{2}\right) \Psi_{\boldsymbol{k}_{1}}^{\alpha_{1}} \Psi_{-\boldsymbol{k}_{1}}^{\alpha_{1}} \Psi_{\boldsymbol{k}_{2}}^{\alpha_{1}} \Psi_{-\boldsymbol{k}_{2}}^{\alpha_{1}}+\mathcal{O}\left(\Psi^{5}, \Omega^{3}\right) .
\end{aligned}
$$

Eq. (C.50) retains a finite excluded volume parameter and the possibility of replicaasymmetric solutions. In the compressible case, the saddle point eq. (3.135) of the density field is proportional to $\Psi$, so that the expansion to fourth order in $\Psi$ is not systematic; the inconsistency is, however, removed for large $\mu$, where $\bar{\Omega} \rightarrow \mathcal{O}\left(\Psi^{2}\right)$. 


\section{C.5. Extended Maxwell construction}

In section 2.5.7 we have a fourth-order Landau free energy of the type

$$
\begin{aligned}
F(\phi)= & a \cdot \frac{1}{V} \int \mathrm{d}^{d} x(\phi(\boldsymbol{x}))^{2}+b \cdot \frac{1}{V} \int \mathrm{d}^{d} x(\phi(\boldsymbol{x}))^{3} \\
& +c \cdot \frac{1}{V} \int \mathrm{d}^{d} x(\phi(\boldsymbol{x}))^{4}+d \cdot\left(\frac{1}{V} \int \mathrm{d}^{d} x(\phi(\boldsymbol{x}))^{2}\right)^{2},
\end{aligned}
$$

describing the separation into macroscopic phases. Due to the nonlocal last term, ordinary Maxwell construction can't be used to determine the minimum of (C.51). Instead we employ an explicit ansatz for the separation into two macroscopic phases with compositions $\phi_{1,2}$ and volume fractions $v_{1,2}$ :

$$
\phi(\boldsymbol{x})= \begin{cases}\phi_{1}, & \boldsymbol{x} \in \mathbb{V}_{1} \\ \phi_{2}, & \boldsymbol{x} \in \mathbb{V}_{2}\end{cases}
$$

where $\left|\mathbb{V}_{1,2}\right|=v_{1,2} V$. The field $\phi(\boldsymbol{x})$ describes the deviation from zero mean; moreover, the melt occupies the whole available volume, hence we can eliminate $v_{1,2}$ with the side conditions

$$
v_{1}+v_{2}=1 \quad \text { and } \quad v_{1} \phi_{1}+v_{2} \phi_{2}=0 .
$$

Minimisation of the free energy (C.51) with respect to $\phi_{1}$ and $\phi_{2}$ leads to the solution $\phi_{1} \phi_{2}=0$ corresponding to the single-phase state and to

$$
\begin{aligned}
& \phi_{1,2}=-\frac{b}{4 c} \pm \frac{1}{4 c} \sqrt{\frac{b^{2}(3 c+d)-8 a c^{2}}{c+d}}, \\
& v_{1,2}=\frac{1}{2} \pm \frac{b}{2 c} \sqrt{\frac{c^{2}(c+d)}{b^{2}(3 c+d)-8 a c^{2}}}
\end{aligned}
$$

corresponding to the phase separated state, in which the free energy is given by

$$
F_{\text {sep }}=-\frac{\left(b^{2}-4 a c\right)^{2}}{64 c^{2}(c+d)}
$$

The two-phase region is defined by $F_{\text {sep }}<0$ or, equivalently, $a<b^{2} / 4 c$. For

$$
a=\frac{b^{2}}{4 c}-\epsilon
$$

the compositions and volume fractions are given to first order in $\epsilon$ by

$$
\begin{aligned}
& v_{1}=1-\frac{2 c^{2}}{b^{2}(c+d)} \epsilon, \quad \phi_{1}=\quad \frac{c}{b(c+d)} \epsilon, \\
& v_{2}=\frac{2 c^{2}}{b^{2}(c+d)} \epsilon \quad \text { and } \quad \phi_{2}=-\frac{b}{2 c}-\frac{c}{b(c+d)} \epsilon \text {. }
\end{aligned}
$$




\section{C.6. Alternative Landau expansion}

In this section we shall derive the Landau expansion of the free energy used in section 2.6. We start from the effective free energy (2.61) in terms of the physical charge and monomer density and the auxiliary fields. First, we integrate out the physical charge density on the saddle point level,

$$
\left.\frac{\partial \mathcal{F}_{n}}{\partial \rho_{-\boldsymbol{k}}^{\alpha}}\right|_{\rho_{\boldsymbol{k}}^{\alpha}=\bar{\rho}_{k}^{\alpha}}=0 \quad \Rightarrow \quad \bar{\rho}_{\boldsymbol{k}}^{\alpha}=\mathrm{i} \frac{2 V}{\mu U_{k}} \hat{\rho}_{\boldsymbol{k}}^{\alpha} .
$$

Insertion into eq. (2.61) and taking the incompressible limit $\mu \rightarrow \infty$ yields

$$
\begin{array}{r}
n \mathcal{F}_{n}\left(\left\{\rho_{\boldsymbol{k}}, \hat{\rho}_{\boldsymbol{k}}, \sigma_{\boldsymbol{k}}, \hat{\sigma}_{\boldsymbol{k}}\right\}\right):=-\mathrm{i} \sum_{\alpha=1}^{n} \sum_{\boldsymbol{k}}^{\prime} \hat{\sigma}_{\boldsymbol{k}}^{\alpha} \sigma_{-\boldsymbol{k}}^{\alpha}-\frac{\chi}{4 V} \sum_{\alpha=1}^{n} \sum_{\boldsymbol{k}}^{\prime} \sigma_{-\boldsymbol{k}}^{\alpha} W_{k} \sigma_{\boldsymbol{k}}^{\alpha} \\
-N \ln \left[\left\langle\exp \left(-\mathrm{i} \sum_{\alpha=1}^{n} \sum_{\boldsymbol{k}}^{\prime} \sum_{s=1}^{L}\left(\hat{\rho}_{\boldsymbol{k}}^{\alpha}+q(s) \cdot \hat{\sigma}_{\boldsymbol{k}}^{\alpha}\right) \mathrm{e}^{-\mathrm{i} \boldsymbol{k} \boldsymbol{r}^{\alpha}(s)}\right)\right\rangle\right]
\end{array}
$$

where we have omitted the constant $\mathcal{C}$ of eq. (2.61). The logarithmic contribution is expanded in powers of $\hat{\rho}$ and $\hat{\sigma}$; subsequently, we trace out the density field $\hat{\rho}$ on the saddle point level. With the substitutions $\Omega_{\boldsymbol{k}}^{\alpha}=\mathrm{i} \cdot 2 V \hat{\rho}_{\boldsymbol{k}}^{\alpha}$ and $\Psi_{\boldsymbol{k}}^{\alpha}=-2 \mathrm{i} V \hat{\sigma}_{\boldsymbol{k}}^{\alpha}$, this task is formally almost identical to expanding, and tracing out $\Omega$ in, eq. (2.69) in the incompressible limit. Hence we can adopt the results of section 2.5 .4 and appendix C.4. Restricting the discussion to the replica-symmetric case $\sigma_{\boldsymbol{k}}^{\alpha}=\sigma_{\boldsymbol{k}}$ and $\hat{\sigma}_{\boldsymbol{k}}^{\alpha}=\hat{\sigma}_{\boldsymbol{k}}$ we obtain, to fourth order in $\hat{\sigma}$,

$$
\begin{aligned}
f:= & \lim _{n \rightarrow 0} \frac{1}{N} \mathcal{F}_{n}\left(\left\{\sigma_{\boldsymbol{k}}, \hat{\sigma}_{\boldsymbol{k}}\right\}\right) \\
= & -\frac{\chi}{4 N V} \sum_{\boldsymbol{k}}^{\prime} \sigma_{-\boldsymbol{k}} W_{k} \sigma_{\boldsymbol{k}}-\frac{\mathrm{i}}{N} \sum_{\boldsymbol{k}}^{\prime} \hat{\sigma}_{\boldsymbol{k}} \sigma_{-\boldsymbol{k}}+\frac{1-q^{2}}{2} \sum_{\boldsymbol{k}}^{\prime} S\left(\boldsymbol{k}^{2}\right) \hat{\sigma}_{\boldsymbol{k}} \hat{\sigma}_{-\boldsymbol{k}} \\
& -\mathrm{i} \frac{q\left(1-q^{2}\right)}{3} \sum_{\boldsymbol{k}_{1,2,3}}^{\prime} S_{3}\left(\boldsymbol{k}_{1}, \boldsymbol{k}_{2}\right) \delta_{\sum_{\nu=1}^{3} \boldsymbol{k}_{\nu}, \mathbf{0}} \hat{\sigma}_{\boldsymbol{k}_{1}} \hat{\sigma}_{\boldsymbol{k}_{2}} \hat{\sigma}_{\boldsymbol{k}_{3}} \\
& +\frac{1}{8} \sum_{\boldsymbol{k}_{1,2,3,4}}^{\prime}\left(\left(1-q^{2}\right)^{2} \frac{S_{2}\left(\boldsymbol{k}_{1}, \boldsymbol{k}_{2}\right) S_{2}\left(\boldsymbol{k}_{3}, \boldsymbol{k}_{4}\right)}{D\left(\left(\boldsymbol{k}_{1}+\boldsymbol{k}_{2}\right)^{2}\right)}-\frac{1}{3} S_{4}\left(\boldsymbol{k}_{1}, \boldsymbol{k}_{2}, \boldsymbol{k}_{3}\right)\right) \\
& \times \delta_{\sum_{\nu=1}^{4} \boldsymbol{k}_{\nu}, \mathbf{0}} \hat{\sigma}_{\boldsymbol{k}_{1}} \hat{\sigma}_{\boldsymbol{k}_{2}} \hat{\sigma}_{\boldsymbol{k}_{3}} \hat{\sigma}_{\boldsymbol{k}_{4}} \\
& +\frac{1}{8} \sum_{\boldsymbol{k}_{1,2}}^{\prime}\left(S_{5}\left(\boldsymbol{k}_{1}, \boldsymbol{k}_{2}\right)-\left(1-q^{2}\right)^{2} S\left(\boldsymbol{k}_{1}^{2}\right) S\left(\boldsymbol{k}_{2}^{2}\right)\right) \hat{\sigma}_{\boldsymbol{k}_{1}} \hat{\sigma}_{-\boldsymbol{k}_{1}} \hat{\sigma}_{\boldsymbol{k}_{2}} \hat{\sigma}_{-\boldsymbol{k}_{2}} .
\end{aligned}
$$

The auxiliary charge density field $\hat{\sigma}$ is eliminated perturbatively, which in the symmetric case $q=0$ yields

$$
\hat{\sigma}_{\boldsymbol{k}}=\frac{\mathrm{i} \sigma_{\boldsymbol{k}}}{N S\left(k^{2}\right)}+\mathcal{O}\left(\sigma^{3}\right)
$$


and

$$
\begin{aligned}
f= & \frac{1}{2 N^{2} M^{2} Q} \sum_{\boldsymbol{k}}^{\prime}\left(\frac{M^{2} Q}{S\left(k^{2}\right)}-\frac{M \varrho_{0} \chi W_{k}}{2}\right) \sigma_{\boldsymbol{k}} \sigma_{-\boldsymbol{k}} \\
& +\frac{1}{8 N^{4}} \sum_{\boldsymbol{k}_{1,2,3,4}}^{\prime} \frac{S_{2}\left(\boldsymbol{k}_{1}, \boldsymbol{k}_{2}\right) S_{2}\left(\boldsymbol{k}_{3}, \boldsymbol{k}_{4}\right) / D\left(\left(\boldsymbol{k}_{1}+\boldsymbol{k}_{2}\right)^{2}\right)-\frac{1}{3} S_{4}\left(\boldsymbol{k}_{1}, \boldsymbol{k}_{2}, \boldsymbol{k}_{3}\right)}{S\left(\boldsymbol{k}_{1}^{2}\right) S\left(\boldsymbol{k}_{2}^{2}\right) S\left(\boldsymbol{k}_{3}^{2}\right) S\left(\boldsymbol{k}_{4}^{2}\right)} \\
& \times \delta_{\sum_{\nu=1}^{4} \boldsymbol{k}_{\nu}, \mathbf{0}} \sigma_{\boldsymbol{k}_{1}} \sigma_{\boldsymbol{k}_{2}} \sigma_{\boldsymbol{k}_{3}} \sigma_{\boldsymbol{k}_{4}} \\
& +\frac{1}{8 N^{4}} \sum_{\boldsymbol{k}_{1,2}} \frac{S_{5}\left(\boldsymbol{k}_{1}, \boldsymbol{k}_{2}\right)-S\left(\boldsymbol{k}_{1}^{2}\right) S\left(\boldsymbol{k}_{2}^{2}\right)}{\left(S\left(\boldsymbol{k}_{1}^{2}\right) S\left(\boldsymbol{k}_{2}^{2}\right)\right)^{2}} \sigma_{\boldsymbol{k}_{1}} \sigma_{-\boldsymbol{k}_{1}} \sigma_{\boldsymbol{k}_{2} \sigma_{-\boldsymbol{k}_{2}}}+\mathcal{O}\left(\sigma^{5}\right) .
\end{aligned}
$$

In the case $q=0$ under consideration, the expansions (C.64) and (2.80) are equivalent in the range of validity, i.e. for small amplitudes $\sigma$ and $\Psi$, respectively. Yet version (C.64), used in a similar form in ref. [17], requires a higher computational effort as can be seen above: besides the derivation of its precursor (C.62), comparable to the derivation of version (2.80), it also requires the perturbative elimination of the auxiliary field. 


\section{Homopolymer blends: Auxiliary materials}

\section{1. Relation between order parameters and auxiliary fields}

To establish the relation between the order parameters defined in section 3.3 and the corresponding fields in the effective free energy (3.60), it is helpful to extend $Z_{n}$ to a generating functional for $Q$ and $S$. We define the replica-Helmholtz free energy $\Phi_{n}(U, W)$ as

$$
\begin{aligned}
& \exp \left(-n \Phi_{n}(U, W)\right):= \\
& \left\langle\operatorname { e x p } \left\{\frac{1}{2 N} \sum_{\alpha=0}^{n} \sum_{\boldsymbol{k}}^{\prime}\left(\chi^{\alpha} S_{\boldsymbol{k}}^{\alpha} S_{-\boldsymbol{k}}^{\alpha}-\tilde{\lambda}^{\alpha} Q_{\boldsymbol{k}}^{\alpha} Q_{-\boldsymbol{k}}^{\alpha}\right)+\frac{\mu}{2 N V^{n}} \overline{\sum_{\hat{k}}} Q_{\hat{k}} Q_{-\hat{k}}\right.\right. \\
& \left.\left.-\frac{1}{N} \sum_{\alpha=0}^{n} \sum_{\boldsymbol{k}}^{\prime}\left(W_{\boldsymbol{k}}^{\alpha} S_{-\boldsymbol{k}}^{\alpha}+U_{\boldsymbol{k}}^{\alpha} Q_{-\boldsymbol{k}}^{\alpha}\right)-\frac{1}{N} \overline{\sum_{\hat{k}}} U_{\hat{k}} Q_{-\hat{k}}\right\}\right\rangle_{n}^{\mathrm{W}}
\end{aligned}
$$

so that $Z_{n}=\mathcal{B}_{n} \cdot \exp \left(-n \Phi_{n}(U=0, W=0)\right)$. The fields $U$ and $W$ are the generators for $Q$ and $S$ and should not be confused with the range of the excluded volume and incompatibility interactions, which have been taken to be delta-shaped. The replica-Helmholtz free energy is related to the free energy via

$$
F=\lim _{n \rightarrow 0} \Phi_{n}(0,0),
$$

where the additive constant originating from $\mathcal{B}_{n}$, the contribution of the zero replica sector, has been neglected. Apart from a modification of the HubbardStratonovich transformations (see appendix A.2) due to the presence of the source fields, the decoupling can be done as before. As pointed out in section 3.4.2, the Fourier components of the order parameters for opposite wavevectors are dependent. Therefore, the auxiliary fields are introduced only for $\boldsymbol{k n}>0$ and $\hat{k} \hat{n}>0$, yielding

$$
\begin{aligned}
& \exp \left\{-n \Phi_{n}(\mu, U, W)\right\} \\
& =\exp \left\{-\frac{1}{2 N} \sum_{\alpha=0}^{n} \sum_{\boldsymbol{k}}^{\prime}\left(\frac{1}{\chi^{\alpha}}\left|W_{\boldsymbol{k}}^{\alpha}\right|^{2}-\frac{1}{\hat{\lambda}^{\alpha}}\left|U_{\boldsymbol{k}}^{\alpha}\right|^{2}\right)-\frac{1}{2 N} \overline{\sum_{\hat{k}}} \frac{1}{V^{-n} \mu}\left|U_{\hat{k}}\right|^{2}\right\} \\
& \quad \int \mathcal{D}_{n} \Psi \mathcal{D}_{n} \Omega \overline{\mathcal{D}} \Omega \exp \left\{-n \mathcal{F}_{n}(\{\Psi, \Omega\})\right. \\
& \times \frac{\left.-\sum_{\alpha=0}^{n} \sum_{\boldsymbol{k}}^{\prime}\left(\frac{1}{\chi^{\alpha}} \Psi_{\boldsymbol{k}}^{\alpha} W_{-\boldsymbol{k}}^{\alpha}+\frac{\mathrm{i}}{\tilde{\lambda}^{\alpha}} \Omega_{\boldsymbol{k}}^{\alpha} U_{-\boldsymbol{k}}^{\alpha}\right)-\overline{\sum_{\hat{k}}} \frac{1}{V^{-n} \mu} \Omega_{\hat{k}} U_{-\hat{k}}\right\}}{\int \mathcal{D} \psi \mathcal{D} \omega \exp \left\{-n \mathcal{F}_{\mathrm{den}}(\{\psi, \omega\})\right\}},
\end{aligned}
$$


with $\mathcal{F}_{n}$ and $\mathcal{F}_{\text {den }}$ defined in eqs. (3.60) and (3.61). The integral measures read

$$
\begin{array}{rlrl}
\mathcal{D} \omega & :=\prod_{\boldsymbol{k}}^{\dagger} \frac{N}{2 \pi \tilde{\lambda}^{0}} \mathrm{~d} \operatorname{Re} \omega_{\boldsymbol{k}} \mathrm{d} \operatorname{Im} \omega_{\boldsymbol{k}} & \mathcal{D} \psi & :=\prod_{\boldsymbol{k}}^{\dagger} \frac{N}{2 \pi \chi_{0}} \mathrm{~d} \operatorname{Re} \psi_{\boldsymbol{k}} \mathrm{d} \operatorname{Im} \psi_{\boldsymbol{k}}, \\
\mathcal{D}_{n} \Omega & :=\prod_{\alpha=0}^{n} \prod_{\boldsymbol{k}}^{\dagger} \frac{N}{2 \pi \tilde{\lambda}^{\alpha}} \mathrm{d} \operatorname{Re} \Omega_{\boldsymbol{k}}^{\alpha} \mathrm{d} \operatorname{Im} \Omega_{\boldsymbol{k}}^{\alpha}, & \mathcal{D}_{n} \Psi:=\prod_{\alpha=0}^{n} \prod_{\boldsymbol{k}}^{\dagger} \frac{N}{2 \pi \chi^{\alpha}} \mathrm{dRe} \Psi_{\boldsymbol{k}}^{\alpha} \mathrm{dIm} \Psi_{\boldsymbol{k}}^{\alpha}
\end{array}
$$

and

$$
\overline{\mathcal{D}} \Omega:=\bar{\prod}_{\hat{k}}^{\dagger} \frac{N \mathrm{~d} \operatorname{Re} \Omega_{\hat{k}} \mathrm{~d} \operatorname{Im} \Omega_{\hat{k}}}{2 \pi V^{-n} \mu},
$$

where $\prod_{\boldsymbol{k}}^{\dagger}$ and $\bar{\prod}_{\hat{k}}^{\dagger}$ denote the products over all nonzero $\boldsymbol{k}$ and higher-replica $\hat{k}$, restricted to half spaces $\boldsymbol{k n}>0$ and $\hat{k} \hat{n}>0$, respectively. The relation between expectation values and moments of the physical order parameters $Q$ and $S$ and those of the associated fields $\Omega$ and $\Psi$ can now be established in two steps.

We first equate the derivatives of the replica-Helmholtz free energy with respect to the source fields $U$ and $W$ taken before and after decoupling, i.e. from eq. (D.65) and from eq. (D.67), evaluated at $U=W=0$. For instance,

$$
\left.\frac{\mathrm{i}}{\tilde{\lambda}^{\alpha}}\left\langle\Omega_{\boldsymbol{k}}^{\alpha}\right\rangle_{n}^{\mathcal{F}} \stackrel{(\mathrm{D} .67)}{=} \frac{\partial \exp \left(-n \Phi_{n}\right)}{\partial U_{-\boldsymbol{k}}^{\alpha}}\right|_{U=W=0} \stackrel{(\mathrm{D.65})}{=} \frac{\left\langle Q_{\boldsymbol{k}}^{\alpha}\right\rangle_{n}^{\mathcal{P}}}{N}
$$

with the average $\langle\ldots\rangle_{n}^{\mathcal{P}}$ with respect to the replicated total Hamiltonian of the uncrosslinked system plus the quasi-Hamiltonian of the constraints,

$$
\langle\cdots\rangle^{\mathcal{P}}:=\frac{\left\langle\cdots \exp \left(-\frac{\mu V}{2 N} \sum_{a, a^{\prime}, i, i^{\prime}} \int_{0}^{1} \mathrm{~d} s \mathrm{~d} s^{\prime} \delta\left(\hat{r}_{a, i}(s)-\hat{r}_{a^{\prime}, i^{\prime}}\left(s^{\prime}\right)\right)\right)\right\rangle_{n}^{\mathrm{T}}}{\left\langle\exp \left(-\frac{\mu V}{2 N} \sum_{a, a^{\prime}, i, i^{\prime}} \int_{0}^{1} \mathrm{~d} s \mathrm{~d} s^{\prime} \delta\left(\hat{r}_{a, i}(s)-\hat{r}_{a^{\prime}, i^{\prime}}\left(s^{\prime}\right)\right)\right)\right\rangle_{n}^{\mathrm{T}}}
$$

The averages $\langle\cdots\rangle_{n}^{\mathcal{P}}$, in turn, are related to disorder averaged thermal expectation values of physical observables $\mathcal{O}\left(\left\{\boldsymbol{r}_{a, i}(s)\right\}\right)$ depending on the monomer positions. In appendix $\mathrm{A}$ of ref. [77] it is shown that

$$
\left[\left\langle\mathcal{O}_{0}\right\rangle_{\mathcal{C}, 0}\left\langle\mathcal{O}_{1}\right\rangle_{\mathcal{C}} \cdots\left\langle\mathcal{O}_{g}\right\rangle_{\mathcal{C}}\right]=\lim _{n \rightarrow 0}\left\langle\mathcal{O}_{0}\left(\left\{\boldsymbol{r}_{a, i}^{0}(s)\right\}\right) \cdots \mathcal{O}_{0}\left(\left\{\boldsymbol{r}_{a, i}^{g}(s)\right\}\right)\right\rangle_{n}^{\mathcal{P}},
$$

where $\left\langle\mathcal{O}_{0}\right\rangle_{\mathcal{C}, 0}$ denotes the thermal average performed at the crosslinking conditions $\left(\lambda_{0}, \chi_{0}\right)$. Eqs. (D.69) and (D.71) imply that

$$
N \cdot \lim _{n \rightarrow 0}\left\langle\Omega_{\boldsymbol{k}}^{\alpha}\right\rangle_{n}^{\mathcal{F}}= \begin{cases}-\mathrm{i}\left(\lambda_{0}-\mu\right)\left[\left\langle Q_{\boldsymbol{k}}\right\rangle_{\mathcal{C}, 0}\right], & \alpha=0, \\ -\mathrm{i}(\lambda-\mu)\left[\left\langle Q_{\boldsymbol{k}}\right\rangle_{\mathcal{C}}\right], & \text { else. }\end{cases}
$$

The averages of $\Omega_{\hat{k}}$ and $\Psi$ and higher-order averages can be transformed similarly. 


\section{D.2. Vertex functions}

In the following, we define and compute the vertex functions appearing in the Landau expansion of the effective free energy (3.63), which are integrals over Wiener correlators of the type

$$
\left\langle\exp \left\{-\mathrm{i} \sum_{\zeta=1}^{z} \hat{k}_{\zeta} \cdot \hat{r}\left(s_{\zeta}\right)\right\}\right\rangle_{n}^{\mathrm{W}}=\delta_{\sum_{\zeta=1}^{z} \hat{k}_{\zeta}, \hat{0}} \exp \left\{\frac{1}{2} \sum_{\zeta, \zeta^{\prime}}\left|s_{\zeta}-s_{\zeta^{\prime}}\right| \hat{k}_{\zeta} \cdot \hat{k}_{\zeta^{\prime}}\right\}
$$

with the Wiener measure defined below eq. (3.63); a derivation of eq. (D.73) can be found in [77]. The correlator vanishes unless the wavevectors sum to zero in each replica. If just single-replica quantities are involved, the correlator factorises,

$$
\begin{aligned}
\left\langle\exp \left\{-\mathrm{i} \sum_{\zeta_{1}=1}^{z_{1}} \boldsymbol{k}_{\zeta_{1}} \cdot \boldsymbol{r}^{\alpha_{1}}\left(s_{\zeta_{1}}\right)-\ldots-\mathrm{i} \sum_{\zeta_{1}=1}^{z_{1}} \boldsymbol{k}_{\zeta_{m}} \cdot \boldsymbol{r}^{\alpha_{m}}\left(s_{\zeta_{m}}\right)\right\}\right\rangle_{n}^{\mathrm{W}}= \\
\left\langle\exp \left\{-\mathrm{i} \sum_{\zeta_{1}=1}^{z_{1}} \boldsymbol{k}_{\zeta_{1}} \boldsymbol{r}\left(s_{\zeta_{1}}\right)\right\}\right\rangle^{\mathrm{W}} \ldots\left\langle\exp \left\{-\mathrm{i} \sum_{\zeta_{1}=1}^{z_{m}} \boldsymbol{k}_{\zeta_{m}} \boldsymbol{r}\left(s_{\zeta_{m}}\right)\right\}\right\rangle^{\mathrm{W}}
\end{aligned}
$$

for pairwise distinct $\alpha_{1}, \ldots, \alpha_{m}$, where $\langle\cdots\rangle^{\mathrm{W}}$ denotes the unreplicated Wiener average.

\section{D.2.1. Definition of the vertex functions}

The second-order coefficients of the Landau expansion are governed by the Debye function

$$
\begin{aligned}
g_{\mathrm{D}}\left(k^{2}\right) & :=\int_{0}^{1} \mathrm{~d} s_{1} \mathrm{~d} s_{2}\left\langle\mathrm{e}^{-\mathrm{i} \boldsymbol{k}\left(\boldsymbol{r}\left(s_{1}\right)-\boldsymbol{r}\left(s_{2}\right)\right)}\right\rangle^{\mathrm{W}}=\int_{0}^{1} \mathrm{~d} s_{1} \mathrm{~d} s_{2}\left\langle\mathrm{e}^{-\mathrm{i} \hat{k}\left(\hat{r}\left(s_{1}\right)-\hat{r}\left(s_{2}\right)\right)}\right\rangle_{n}^{\mathrm{W}} \\
& =\frac{\mathrm{e}^{-k^{2}}-1+k^{2}}{k^{4} / 2}=1-\frac{1}{3} k^{2}+\frac{1}{12} k^{4}+\mathcal{O}\left(k^{6}\right)
\end{aligned}
$$

the scattering function for a non-interacting Gaussian chain.

The third-order correlators

$$
\begin{aligned}
g_{3}\left(\boldsymbol{k}_{1}, \boldsymbol{k}_{2}\right):=\int_{0}^{1} \mathrm{~d} s_{1} \mathrm{~d} s_{2} \mathrm{~d} s_{3} & \left.\left\langle\mathrm{e}^{-\mathrm{i} \sum_{\nu=1}^{3} \boldsymbol{k}_{\nu} \boldsymbol{r}\left(s_{\nu}\right)}\right\rangle^{\mathrm{W}}\right|_{\boldsymbol{k}_{3}=-\boldsymbol{k}_{1}-\boldsymbol{k}_{2}} \\
= & \left.\int_{0}^{1} \mathrm{~d} s_{1} \mathrm{~d} s_{2} \mathrm{~d} s_{3}\left\langle\mathrm{e}^{-\mathrm{i} \sum_{\nu=1}^{3} \hat{k}_{\nu} \hat{r}\left(s_{\nu}\right)}\right\rangle^{\mathrm{W}}\right|_{\hat{k}_{3}=-\hat{k}_{1}-\hat{k}_{2}}
\end{aligned}
$$

and

$$
\begin{aligned}
& g_{\Psi^{2} \Omega}\left(\boldsymbol{k}_{1}, \boldsymbol{k}_{2}\right):=\int_{0}^{1} \mathrm{~d} s_{1} \mathrm{~d} s_{2} \mathrm{~d} s_{3}\left\langle\mathrm{e}^{-\mathrm{i} \boldsymbol{k}_{1}\left(\boldsymbol{r}\left(s_{1}\right)-\boldsymbol{r}\left(s_{3}\right)\right)}\right\rangle^{\mathrm{W}}\left\langle\mathrm{e}^{-\mathrm{i} \boldsymbol{k}_{2}\left(\boldsymbol{r}\left(s_{2}\right)-\boldsymbol{r}\left(s_{3}\right)\right)}\right\rangle^{\mathrm{W}}
\end{aligned}
$$

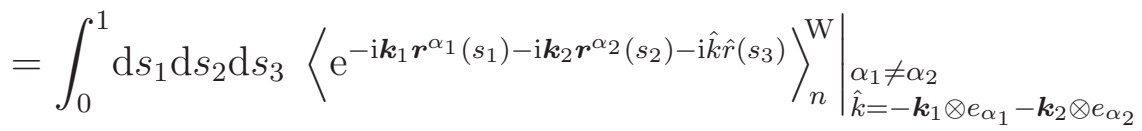


describe the correlation between three one-replica fields and the correlation between two one-replica-fields and a higher-order replica field, respectively.

Finally, the fourth-order correlator is given by

$$
g_{\Psi^{4}}\left(\boldsymbol{k}_{1}, \boldsymbol{k}_{2}, \boldsymbol{k}_{3}\right)=\left.\int_{0}^{1} \mathrm{~d} s_{1} \mathrm{~d} s_{2} \mathrm{~d} s_{3} \mathrm{~d} s_{4}\left\langle\mathrm{e}^{-\mathrm{i} \sum_{\nu=1}^{4} \boldsymbol{k}_{\nu} \boldsymbol{r}\left(s_{\nu}\right)}\right\rangle^{\mathrm{W}}\right|_{\boldsymbol{k}_{4}=-\sum_{\nu=1}^{3} \boldsymbol{k}_{\nu}} .
$$

Lamellar case The third- and fourth-order correlators depend on the direction of the wavevectors. In particular, we require the "lamellar" case, in which all wavevectors are collinear. We note that $g_{3}(\boldsymbol{k},-\boldsymbol{k})=g_{\mathrm{D}}\left(k^{2}\right)$ and define

$$
\begin{aligned}
g_{3}\left(k^{2}\right) & :=g_{3}(\boldsymbol{k}, \boldsymbol{k})=\frac{-\left(\mathrm{e}^{-4 k^{2}}-1+4 k^{2}-8 k^{4}\right)+64\left(\mathrm{e}^{-k^{2}}-1+k^{2}-\frac{1}{2} k^{4}\right)}{12 k^{6}} \\
& =1-k^{2}+\frac{3}{4} k^{4}+\mathcal{O}\left(k^{6}\right), \\
g_{\Psi^{2} \Omega}\left(k^{2}\right) & :=g_{\Psi^{2} \Omega}(\boldsymbol{k},-\boldsymbol{k})=\frac{-\mathrm{e}^{-2 k^{2}}+\left(8+2 k^{2}\right) \mathrm{e}^{-k^{2}}+4 k^{2}-7}{k^{6}} \\
& =1-\frac{2}{3} k^{2}+\frac{17}{60} k^{4}+\mathcal{O}\left(k^{6}\right)
\end{aligned}
$$

and

$$
\begin{aligned}
g_{\Psi^{4}}\left(k^{2}\right) & :=g_{\Psi^{4}}(\boldsymbol{k}, \boldsymbol{k},-\boldsymbol{k}) \\
& =\frac{144 k^{4}-60 k^{2}\left(9+4 \mathrm{e}^{-k^{2}}\right)+784\left(1-\mathrm{e}^{-k^{2}}\right)-\left(1-\mathrm{e}^{-4 k^{2}}\right)}{18 k^{8}} \\
& =1-\frac{2}{3} k^{2}+\frac{11}{30} k^{4}+\mathcal{O}\left(k^{4}\right) .
\end{aligned}
$$

\section{D.3. Scaling function for the gelation order parameter}

The localisation lengths $\tau$ of monomers in the gel fraction of a crosslinked homopolymer melt or blend are distributed according to the distribution $p(\tau)$; see section 3.5.3. The fraction of the gel and the distribution are determined from the self-consistent solution of the saddle-point equations (3.76) with the order parameter hypothesis (3.80). The gelation order parameter, essentially the Laplace transform of $p(\tau)$, is proportional to a scaling function $\omega(x)$, which is computed in [77] in the asymptotic regimes of small and large $x$. For convenience, we define a rescaled version of $\omega$,

$$
w\left(k^{2}\right):=2 \cdot \omega\left(\sqrt{8 k^{2} / 3}\right),
$$

This also absorbs a factor of two arising from the different length-scale used in [77] (the Wiener Hamiltonian used therein differs from eq. (3.10) by a factor of two). 
Figure D.2: $\quad$ Scaling function $\omega(k)$ versus $k$ : Asymptotic expressions and interpolated function.

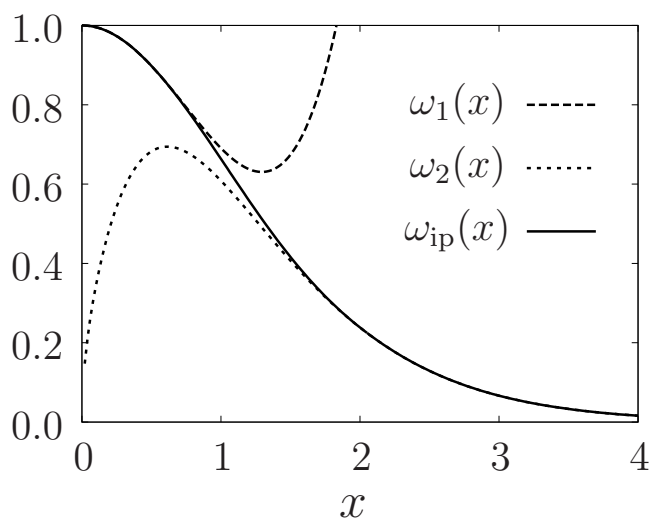

\section{D.3.1. Interpolation formula}

The scaling function $\omega(k)$ defined in [77] can be described asymptotically by

$$
\omega(x) \approx \begin{cases}\omega_{1}(x):=1-0.4409 x^{2}+0.1316 x^{4} & \text { for } x \ll 1 \\ \omega_{2}(x):=3\left(\frac{\pi^{2} x^{6}}{8 \cdot 1.678}\right)^{1 / 4} \mathrm{e}^{-\sqrt{2 \cdot 1.678} x}\left(1+\frac{27}{40 \sqrt{2 \cdot 1.678} x}\right) & \text { for } x \gg 1 .\end{cases}
$$

In order to access the whole range of $0<x<\infty$ we interpolate between the asymptotic regimes using the interpolation formula

$$
\omega(k) \approx \begin{cases}\omega_{1}(x), & \text { for } x<\frac{1}{2} \\ \omega_{\mathrm{ip}}(x), & \text { for } \frac{1}{2} \leq x<2 \\ \omega_{2}(x), & \text { for } x \geq 2\end{cases}
$$

with the interpolating rational function

$$
\omega_{\text {ip }}:=\frac{b_{0}+b_{1} x}{1+a_{1} x+a_{2} x^{2}+a_{3} x^{3}} .
$$

The coefficients $a_{1}=-0.055, a_{2}=0.165, a_{3}=0.139, b_{0}=1.023$ and $b_{1}=-0.194$ are chosen such that the value and first derivative of $\omega_{\text {ip }}(x)$ coincide with those of $\omega_{1}(x)$ at $x=1 / 2$ and with those of $\omega_{2}(x)$ at $x=2$; an additional sampling point is the numerical value $\omega(x=1)=0.664$.

\section{D.4. Hessian matrix of the effective free energy}

The stability considerations and the calculation of charge density fluctuations are based on the matrix of the second derivatives of $\mathcal{F}_{n}$ with respect to the charge density fields $\Psi_{\boldsymbol{k}}^{\alpha}$. This Hessian matrix $A \in \mathbb{R}^{(n+1) \times(n+1)}$ is of the form

$$
\mathbf{A}=\left(\begin{array}{cccc}
c & -b & \cdots & -b \\
-b & a & \ddots & \vdots \\
\vdots & \ddots & \ddots & -b \\
-b & \cdots & -b & a
\end{array}\right)
$$




\section{D.4.1. Eigenvalue spectrum}

For integer $n>1$, the eigenvalues of $\mathbf{A}$ are $a+b$, which is $(n-1)$-fold degenerate, and $\left(a+(1-n) b+c \pm \sqrt{(a+b(1-n)-c)^{2}+4 n b^{2}}\right) / 2$, both non-degenerate. The corresponding eigenvectors read $(0,1,-1,0, \ldots, 0)^{T},(0,0,1,-1,0, \ldots, 0)^{T}$, $\ldots,(0, \ldots, 0,1,-1)^{T}$ and $\left(\frac{1}{2 b}\left(a+(1-n) b-c \pm \sqrt{(a+b(1-n)-c)^{2}+4 n b^{2}}\right), 1, \ldots, 1\right)^{T}$, respectively. In the replica-limit $n \rightarrow 0$, the eigenvalues read

$$
\begin{array}{ll}
\lambda_{1}=c \quad \text { and } \quad \lambda_{2}=a+b,
\end{array}
$$

which are non- and $n$-fold degenerate, respectively.

\section{D.4.2. Inverse matrix}

The inverse matrix of $\mathbf{A}$ is given by

$$
\mathbf{A}^{-1}=\left(\begin{array}{ccccc}
\mathfrak{c} & \mathfrak{d} & \mathfrak{d} & \cdots & \mathfrak{d} \\
\mathfrak{d} & \mathfrak{a} & \mathfrak{b} & \cdots & \mathfrak{b} \\
\mathfrak{d} & \mathfrak{b} & \mathfrak{a} & \ddots & \vdots \\
\vdots & \vdots & \ddots & \ddots & \mathfrak{b} \\
\mathfrak{d} & \mathfrak{b} & \cdots & \mathfrak{b} & \mathfrak{a}
\end{array}\right)
$$

where

$$
\begin{aligned}
\mathfrak{a} & =\frac{a c+(2-n) b c+(1-n) b^{2}}{(1-n) b^{2} c+(2-n) a b c-n b^{2}(a+b)+a^{2} c} & \stackrel{n \rightarrow 0}{\longrightarrow} \frac{a c+2 b c+b^{2}}{(a+b)^{2} c}, \\
\mathfrak{b} & =\frac{b(c+b)}{(1-n) b^{2} c+(2-n) a b c-n b^{2}(a+b)+a^{2} c} & \stackrel{n \rightarrow 0}{\longrightarrow} \frac{b(c+b)}{(a+b)^{2} c} \\
\mathfrak{c} & =\frac{a+(1-n) b}{a c+(1-n) b c-n b^{2}} & \stackrel{n \rightarrow 0}{\longrightarrow} \frac{1}{c}
\end{aligned}
$$

and

$$
\mathfrak{d}=\frac{b}{a c+(1-n) b c-n b^{2}} \quad \stackrel{n \rightarrow 0}{\longrightarrow} \frac{b}{(a+b) c} .
$$


Appendix 


\section{Bibliography}

[1] Strobl G.R. The Physics of Polymers. Springer-Verlag, Berlin (1996).

[2] Bucknall C.B. Applications of microscopy to the deformation and fracture of rubber-toughened polymers. Journal of Microscopy 201(2), 221 (2001).

[3] LeIBLER L. Theory of microphase separation in block copolymers. Macromolecules 13, 1602 (1980).

[4] JanerT P.K. and Schick M. Phase Behavior of Ternary Homopolymer/Diblock Blends: Microphase Unbinding in the Symmetric System. Macromolecules 30(13), 3916 (1997).

[5] Bates F.S., Rosedale J.H. and Fredrickson G.H. Fluctuation-Induced First-Order Transition of an Isotropic System to a Periodic State. Physical Review Letters 61(19), 2229 (1988).

[6] Mayes A.M. and Olvera de la Cruz M. Microphase separation in multiblock copolymer melts. Journal of Chemical Physics 91(11), 7228 (1989).

[7] Hashimoto T., Shibayama M. and Kawai H. Domain-Boundary Structure of Styrene-Isoprene Block Copolymer Films Cast from Solution. 4. MolecularWeight Dependence of Lamellar Microdomains. Macromolecules 13(5), 1237 (1980).

[8] Förster S., Khandpur A.K., Zhao J., Bates F.S., Hamley I.W., RYAN A.J. and Bras W. Complex Phase Behaviour of PolyisoprenePolystyrene Diblock Copolymers Near the Order-Disorder Transition. Macromolecules 27(23), 6922 (1994).

[9] Khandpur A.K., Förster S., Bates F.S., Hamley I.W., Ryan A.J., Bras W., Almdal K. and Mortensen K. Polyisoprene-Polystyrene Diblock Copolymers Phase Diagram near the Order-Disorder Transition. Macromolecules 28(26), 8796 (1995).

[10] Hashimoto T., Tanaka H. and Hasegawa H. Ordered Structures in Mixtures of a Block Copolymer and Homopolymers. 2. Effects of Molecular Weights of Homopolymers. Macromolecules 23(20), 4378 (1990). 
[11] Bates F.S., Schulz M.F., Khandpur A.K., Förster S., Rosedale J.H., Almdal K. and Mortensen K. Fluctuations, Conformational Asymmetry and Block Copolymer Phase Behaviour. Faraday Discussions 98, 7 (1994).

[12] Bates F.S. Polymer-Polymer Phase Behaviour. Science 251, 898 (1991).

[13] Scott R.L. Thermodynamics of High Polymer Solutions. VI. The Compatibility of Copolymers. Journal of Polymer Science 9(5), 423 (1952).

[14] Flory P.J. Principles of Polymer Chemistry. Cornell University Press, Ithaka (1953).

[15] BAUER B.J. Equilibrium phase compositions of heterogeneous copolymers. Polymer engineering and science 25(17), 1081 (1985).

[16] Nesarikar A., Olvera de la Cruz M. and Crist B. Phase transitions in random copolymers. Journal of Chemical Physics 98(9), 7385 (1993).

[17] Fredrickson G.H., Milner S.T. and Leibler L. Multicritical phenomena and microphase ordering in random copolymer melts. Macromolecules 25(23), 6341 (1992).

[18] Shakhnovich E.I. and Gutin A.M. Formation of microdomains in a quenched disordered heteropolymer. Journal de Physique 50(14), 1843 (1989).

[19] Fredrickson G.H. and Milner S.T. Thermodynamics of Random Copolymer Melts. Physical Review Letters 67(7), 835 (1991).

[20] Houdayer J. and Müller M. Deviations from the mean-field predictions for the phase behaviour of random copolymers melts. Europhysics Letters 58(5), 660 (2002).

[21] Houdayer J. and Müller M. Phase Diagram of Random Copolymer Melts: A Computer Simulation Study. Macromolecules 37(11), 4283 (2004).

[22] Subbotin A.V. and Semenov A.N. Phase equilibria in random multiblock copolymers. Europhysics Letters 7, 49 (2002).

[23] Sfatos C.D., Gutin A.M. and Shakhnovich E.I. Fluctuation effects of microphase separation in a random copolymer Hamiltonian. Journal of Physics A 27(L411-L416) (1994).

[24] Dobrynin A.V. and Erukhomivich I.Y. Flucuation Theory of Random Copolymers. Journal de Physique I 5(3), 365 (1995).

[25] Gutin A.M., Sfatos C.D. and Shakhnovich E.I. Fluctuation effects on microphase separation in random copolymers. Journal of Physics A 27, 7957 (1994). 
[26] Sfatos C.D., Gutin A.M. and Shakhnovich E.I. Phase diagram of random copolymers. Physical Review E 48(1), 465 (1993).

[27] Mirabella, Jr. F.M., Westphal S.P., Fernando P.L. and Ford E.A. Morphological Explanation of the Extraordinary Fracture Toughness of Linear Low Density Polyethylenes. Journal of Polymer Science B 26, 1995 (1988).

[28] A. Nesarikar B.C. and Davidovich A. Liquid-Liquid Phase Separation in Linear Low-Density Polyethylene. Journal of Polymer Science B 32, 641 (1994).

[29] Xenidou M., Beyer F.L., Hadjichristidis N., Gido S.P. and Beck TAN N. Morphology of Model Graft Copolymers with Randomly Placed Trifunctional and Tetrafunctional Branch Points. Macromolecules 31(22), 765 (1998).

[30] Eitouni H.B., Rappl T.J., Gomez E.D., Balsara N.P., Qi S., Chakraborty A.K., Frèchet J.M.J. and Pople J.A. Signatures of the Order-Disorder Transition in Copolymers with Quenched Sequence Disorder. Macromolecules 37(23), 2004 (2004).

[31] Ryan A.J., Macosko C.W. and Bras W. Order-Disorder Transition in a Block Copolyurethane. Macromolecules 25(23), 6277 (1992).

[32] Qi S., Chakraborty Q.K. and Balsara N.P. Microphase separation in molten randomly grafted copolymers. Journal of Chemical Physics 115(7), 3387 (2001).

[33] Velankar S. and Cooper S.L. Microphase Separation and Rheological Properties of Polyurethane Melts. 2. Effect of Block Incompatibility on the Microstructure. Macromolecules 33(2), 382 (2000).

[34] Flory P.J. Statistical Mechanics of Chain Molecules. Interscience Publishers - John Wiley and Sons, New York (1969).

[35] Kunn W. Über die Gestalt fadenförmiger Moleküle in Lösungen. KolloidZeitschrift 68(1), 2 (1934).

[36] Doi M. and Edwards S.F. The Theory of Polymer Dynamics. International series of monographs on physics. Oxford Science Publications, Oxford (1986).

[37] Huggins M.L. Solutions of Long Chain Compounds. Journal of Chemical Physics 9(5), 440 (1941).

[38] Flory P.J. Thermodynamics of High Polymer Solutions. Journal of Chemical Physics 9(8), 660 (1941). 
[39] Flory P.J. Thermodynamics of High Polymer Solutions. Journal of Chemical Physics 10(1), 51 (1942).

[40] Huggins M.L. Thermodynamic properties of solutions of long chain compounds. Annals of the New York Academy of Sciences XLIII, 1 (1942).

[41] Holyst R. and Schick M. Copolymers as amphiphiles in ternary mixtures: An analysis employing disorder, equimaxima and Lifshitz lines. Journal of Chemical Physics 96(10), 7728 (1992).

[42] Krause S. Polymer Compatibility. Journal of Macromolecular Science Reviews in Macromolecular Chemistry C 7(2), 251 (1972).

[43] De Gennes P.G. Qualitative features of polymer demixtion. Journal de Physique Lettres 38(21), L-441 (1977).

[44] BrazovskiI S.A. Fazovy̆ perekhod izotropnoŭ sistemy v neodnorodnoe sostoyanie. Zhurnal eksperimentalnoŭ i teoreticheskoy fiziki 68, 175 (1975). English translation: Phase transition of an isotropic system to a nonuniform state. Soviet Physics - JETP 41, 85 (1975).

[45] De Gennes P.G. Effects of cross-links on a mixture of polymers. Journal de Physique Lettres 40(4), L-69 (1979).

[46] Manson J.A. and Sperling L.H. Polymer blends and composites. Plenum Press, New York (1976).

[47] Paul D.R. and Newman S. (editors). Polymer blends, volume 1+2. Academic Press, New York (1978).

[48] Olabisi O., Robeson L.M. and Shaw M.T. Polymer-Polymer miscibility. Academic Press, New York (1979).

[49] Briber R.M. and BAuer B.J. Effect of Cross-Links on the Phase Separation Behavior of a Miscible Polymer Blend. Macromolecules 21(11), 3296 (1988).

[50] Jinnai H., Hasegawa H., Hashimoto T., Briber R.M. and Han C.C. Effects of Cross-Links on the miscibility of a Deuterated Poylbutadiene and Protonated Polybutadiene Blend. Macromolecules 26(1), 182 (1993).

[51] Benmouna M., Vilgis T.A., Daoud M. and Benhamou M. Scattering and Phase Behaviour of Cross-Linked Blends. Macromolecules 27(5), 1172 (1994).

[52] Bettachy A., Derouiche A. and Benhamou M. Scattered intensity by a cross-linked polymer blend. Macromolecular Theory and Simulations 4, 67 (1995). 
[53] Read D.J., Brereton M.G. and McLeish T.C.B. Theory of the OrderDisorder Phase transition in Cross-Linked Polymer Blends. Journal de Physique II 5(11), 1679 (1995).

[54] Panyukov S. and Rabin Y. Statistical physics of polymer gels. Physics Reports 269, 1 (1996).

[55] Sfatos C.D. and Shakhnovich E.I. Statistical mechanics of random heteropolymers. Physics Reports 288, 77 (1997).

[56] Lay S., J.-U. Sommer and Blumen A. Monte Carlo study of the microphase separation of cross-linked polymer blends. Journal of Chemical Physics 113(24), 11355 (2000).

[57] Benhamou M. Field-theoretical apporoach to critical microphase behavior of symmetric crosslinked polymer blends. Journal of Chemical Physics 102(14), 5854 (1995).

[58] Panyukov S. and Rubinstein M. Stress-Induced Ordering in MicrophaseSeparated Multicomponent Networks. Macromolecules 29(25), 8220 (1996).

[59] Iván B., Almdal K., Mortensen K., Johannsen I. and Kops J. Synthesis, Characterization, and Structural Investigation of Poly(ethylacrylate)-lpolyisobutylene Bicomponent Conetwork. Macromolecules 34(6), 1579 (2001).

[60] Stepanow S., Schulz M. and Binder K. Effects of inhomogeneities of cross-links on a microphase separation of polymer mixtures. Journal de Physique II 4(5), 819 (1994).

[61] Massunaga M.S.O., Paniconi M. and Oono Y. Phenomenological model for cross-linked polymer blends. Physical Review E 56(1), 723 (1997).

[62] Derouiche A., Bettachy A., Benhamou M. and Daoud M. Kinetics of Microphase Separation in Cross-Linked Polymer Blends. Macromolecules 25(26), 7188 (1992).

[63] Daoud M. Phase and microphase separation in polymer blends. Journal de Physique IV - Colloque C1 3(211-223) (1993).

[64] Benhamou M., Derouiche A. and Bettachy A. Microphase separation of crosslinked polymer blends in solution. Journal of Chemical Physics 106(6), 2513 (1996).

[65] Vargas E. and Barbosa M.C. Phase diagram for irregular and nonsymmetric cross-linked polymer blends. Physica A 257, 312 (1998).

[66] Binder K. and Frisch H.L. Phase stability of weakly crosslinked interpenetrating polymer networks. Journal of Chemical Physics 81(4), 2126 (1984). 
[67] Brulet A., Daoud M., Zhou P. and Frisch H.L. Small angle neutron scattering by interpenetrating polymer networks of poly(carbonate-urethane) and polyvinyl pyridine. Journal de Physique II 3(7), 1161 (1993).

[68] Schulz M. Mean field theory of the microphase separation in interpenetrating networks. Journal of Chemical Physics 98(8), 5631 (1992). Author's name misspelled as "Shulz" in the article.

[69] Schulz M. and Frisch H.L. Mean field theory of interpenetrating polymer network gels. Journal of Chemical Physics 107(7), 2673 (1997).

[70] Imagawa A. and Tran-Cong Q. Structure-Property Relationship of Polymer Blends with Co-Continuous Structures Prepared by Photo-Cross-Linking. Macromolecules 28(24), 8388 (1995).

[71] Kataoka K., Harada A., Tamai T. and Tran-Cong Q. Phase Behavior and Photocrosslinking Kinetics of Semi-Interpenetrating Polymer Networks Prepared from Miscible Polymer Blends. Journal of Polymer Science B: Polymer Physics 36, 455 (1998).

[72] Lay S., J.-U. Sommer and Blumen A. Comparison of structural properties of different polymer network types as obtained by computer simulation. Journal of Chemical Physics 110(24), 12173 (1999).

[73] UCHIDA N. Pattern formation in microphase-separating copolymer gels. Journal of Physics: Condensed Matter 16(4), L21 (2004).

[74] Pfahl S. Nematische Elastomere - ein mikroskopisches Modell. Diplomarbeit, Universität Göttingen (2005).

[75] Benmouna F., Maschke U., Coqueret X. and Benmouna M. Theoretical Phase Behaviour of Crosslinked Polymers and Liquid Crystals. Macromolecular Theory and Simulations 10, 63 (2001).

[76] Castillo H.E., Goldbart P.M. and Zippelius A. Distribution of Localisation Lengths in Randomly Crosslinked Macromolecular Network. Europhysics Letters 28(7), 519 (1994).

[77] Goldbart P.M., Castillo H.E. and Zippelius A. Randomly crosslinked macromolecular systems: vulcanization transition to and properties of the amorphous solid state. Advances in Physics 45(5), 393 (1996).

[78] Wald C., Zippelius A. and Goldbart P.M. Glassy states and microphase separation in cross-linked homopolymer blends. Europhysics Letters 70(6), $843(2005)$. 
[79] Wald C., Goldbart P.M. and Zippelius A. Glassy correlations and microstructures in randomly crosslinked homopolymer blends (2005). arXiv: cond-mat/0509694; submitted to the Journal of Chemical Physics.

[80] Deam R.T. and Edwards S.F. The theory of rubber elasticity. Philosophical transactions of the Royal Society of London A 280, 317 (1976).

[81] Edwards S.F. and Anderson P.W. Theory of spin glasses. Journal of Physics F 5(5), 965 (1975).

[82] Mezei F. Neutron scattering and collective dynamics in liquids and glass. In: Liquides, crystallisation et transition vitreuse, Part II - Les Houches Session LI, edited by J.P. Hansen, D. Levesque and J. Zinn-Justin. North-Holland, Amsterdam (1989).

[83] Higgins J.S. and Benoit H.C. Polymers and Neutron Scattering. Clarendon Press, Oxford (1994).

[84] Alexander L.E. X-Ray Diffraction Methods in Polymer Science. WileyInterscience, New York (1969).

[85] De Gennes P.G. Theory of X-ray scattering by liquid macromolecules with heavy atom labels. Journal de Physique 31(2-3), 235 (1969).

[86] Alexander S. and McTague J. Should All Crystals Be bcc? Landau Theory of Solidification and Crystal Nucleation. Physical Review Letters 41(10), 702 (1978).

[87] Fredrickson G.H. and Helfand E. Fluctuation effects in the theory of microphase separation in block copolymers. Journal of Chemical Physics 87(1), 697 (1987).

[88] Mézard M., Parisi G. and Virasoro M.A. Spin glass theory and beyond. World Scientific, Singapore (1987). 
Bibliography

136 


\section{Acknowledgement}

Zum Abschluss möchte ich denjenigen meinen Dank aussprechen, die mich bei der Anfertigung dieser Arbeit unterstützt haben. An erster Stelle möchte ich mich herzlich bei Prof. Annette Zippelius für die motivierende Betreuung, die Unterstützung und ihre ständige Gesprächsbereitschaft bedanken. Ein herzlicher Dank geht auch an Prof. Paul M. Goldbart für die Gastfreundschaft und die angenehme Zusammenarbeit. Herrn Prof. Tim Salditt danke ich für das Interesse an meiner Arbeit und die Übernahme des Korreferats. Besonders danke ich Priv. Doz. Dr. Peter Müller und Dr. Timo Aspelmeier für ihre unerschöpfliche Hilfsbereitschaft und die interessanten physikalischen Diskussionen. Timo Aspelmeier und mein Vater haben mir durch das sorgfältige Korrekturlesen der Arbeit einen großen Dienst erwiesen.

Ein besonderer Dank gilt Gisela Mesecke und den anderen Mitarbeiterinnen des Institutes für die zahlreichen kleinen und großen und Hilfen und ihr Engagement für das Institutsleben.

Ein großes Dankeschön geht an meine Freunde und Kollegen aus der Mensarunde, Garrit, Timo, Peter, Henning, Wolfgang, Stephan, Axel und all die anderen.

Diese Arbeit wurde von der DFG gefördert durch Mittel aus dem LeibnizProgramm, AZ Zi 209/6-1, durch ein Stipendium des Graduiertenkollegs 782 und durch den Sonderforschungsbereich 602. 
Acknowledgement

138 


\title{
Curriculum vitae
}

\author{
Christian Wald \\ Hauptstraße 14a \\ D-37083 Göttingen
}

\section{Biographische Daten}

\author{
Geburtsdatum: $\quad$ 4. September 1974 \\ Geburtsort: Bremen \\ Staatsangehörigkeit: deutsch
}

\section{Bildungsgang}

06/1994 Abitur am Gymnasium Syke

10/1997 Vordiplom in Physik an der Universität Göttingen

2000-2001 Diplomarbeit über rauschinduzierten Transport gekoppelter Teilchen, betreut durch Prof. Dr. Andreas Mielke am Institut für Theoretische Physik der Universität Heidelberg.

02/2001 Diplom in Physik an der Universität Heidelberg

2001-2005 Doktorarbeit, betreut durch Prof. Dr. Annette Zippelius am Institut für Theoretische Physik der Universität Göttingen

10/2004 vierwöchiger Forschungsaufenthalt in der Arbeitsgruppe von Prof. Dr. Paul M. Goldbart, Department of Physics, University of Illinois at Urbana-Champaign (USA)

11/2005 Promotion in Physik an der Universität Göttingen

\section{Auszeichnungen}

07/2001 Otto-Haxel-Preis der Fakultät für Physik und Astronomie der Universität Heidelberg, verliehen "für eine herausragende Diplomarbeit“

Göttingen, 4. Oktober 2005 